Supporting Information for

\title{
Carboxyl Group-Directed Iridium-Catalyzed Enantioselective Hydrogenation of Aliphatic $\gamma$-Ketoacids
}

Mao-Lin $\mathrm{Li}^{\ddagger}$, Yao Li ${ }^{\ddagger}$, Jia-Bin Pan, Yi-Hao Li, Song Song, Shou-Fei Zhu*, Qi-Lin Zhou* State Key Laboratory and Institute of Elemento-Organic Chemistry, College of Chemistry, Nankai University, Tianjin 300071, China

\$These two authors contribute equally

*Correspondence to: sfzhu@nankai.edu.cn; qlzhou@nankai.edu.cn

\section{Table of Contents}

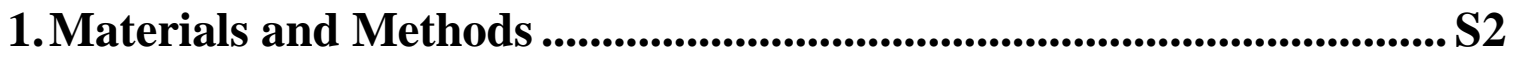

2. Preparation and Analytical Data of Iridium Catalysts.........................S4

3. Preparation and Analytical Data of Substrates .................................... S6

4. General Procedure and Analytical Data of Hydrogenation .................S8

5. Mechanism Experiments and NMR Studies. ........................................ S15

6. NMR Spectra of New Compounds ............................................................ S17

7. GC and HPLC Charts of Hydrogenation Products ..............................S30

8. Computational Data .....................................................................54

The Cartesian coordinates $(\AA)$ for the optimized structures................. S52

The Cartesian coordinates $(\AA)$ for the optimized structures in SI..... S117

9. References................................................................................................S179 


\section{Materials and Methods}

All the air or moisture sensitive reactions and manipulations were performed under an argon atmosphere by using standard Schlenk techniques and a Vacuum Atmospheres Drybox (VAC DRI-LAB HE 493). Melting points were measured on a RY-I apparatus and uncorrected. ${ }^{1} \mathrm{H},{ }^{13} \mathrm{C}$ NMR and ${ }^{31} \mathrm{P}$ NMR spectra were recorded on a Brucker AV 400 spectrometers. Chemical shifts were reported in ppm down field from internal Me4Si, and external $85 \% \mathrm{H}_{3} \mathrm{PO}_{4}$, respectively. Mass spectra were recorded on IonSpec FT-ICR mass spectrometer with ESI or MAIDI resource. Enantiomeric excesses of the asymmetric hydrogenation products were determined by chiral GC, HPLC or SFC. GC analyses were performed using a Hewlett Packard Model HP 6890 Series instruments. HPLC analyses were performed using a Hewlett Packard Model HP 1100 Series instruments. SFC analyses were performed using a Mettler-Toledo Model Analytix SFC. Anhydrous $\mathrm{Et}_{2} \mathrm{O}$, THF and toluene were distilled from sodium benzophenone ketyl. Anhydrous $\mathrm{CH}_{2} \mathrm{Cl}_{2}, \mathrm{NEt}_{3}$ and DMF were freshly distilled from calcium hydride under argon atmosphere. Anhydrous $\mathrm{MeOH}$ was distilled from magnesium under argon atmosphere. $[\operatorname{Ir}(\mathrm{COD}) \mathrm{Cl}]_{2}$ was purchased from Sigma-Aldrich Co. and used as received. Hydrogen gas (99.999\%) was purchased from Boc Gas Inc., Tianjin.
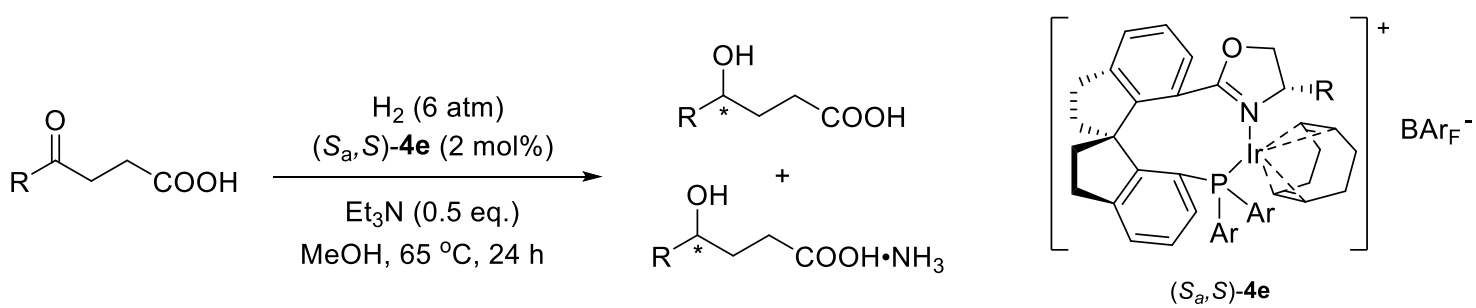

$\mathrm{Ar}=3,5-{ }^{\mathrm{t}} \mathrm{Bu}_{2} \mathrm{C}_{6} \mathrm{H}_{3}, \mathrm{R}=\mathrm{CH}_{2}-1-\mathrm{Np}$

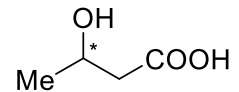

No reaction ${ }^{\mathrm{a}}$<smiles>O=C1CC[C@H](c2ccccc2)O1</smiles>

$94 \%$ yield, $80 \%$ ee

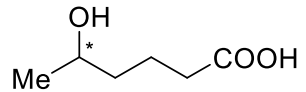

$20 \%$ yield, $35 \%$ ee ${ }^{\text {b }}$

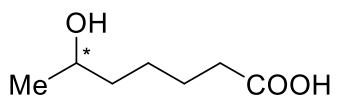

$18 \%$ yield, $16 \%$ ee $^{\mathrm{b}}$

Figure S1. Other substrates for the hydrogenation reaction. Reaction conditions: 0.25 mmol scale, [substrate] $=0.125 \mathrm{~mol} / \mathrm{L},\left(S_{\mathrm{a}}, S\right)-4 \mathrm{e}(2 \mathrm{~mol} \%), \mathrm{H}_{2}(6 \mathrm{~atm}), \mathrm{Et}_{3} \mathrm{~N}$ (0.5 equiv). Full conversions were obtained within $24 \mathrm{~h}$. The yield and ee value were measured after conversion to lactones. ${ }^{a}$ The reaction system is complex and there is no product. ${ }^{b}$ After the carboxyl group was converted into a benzyl ester group, the enantioselectivities were determined by HPLC. 


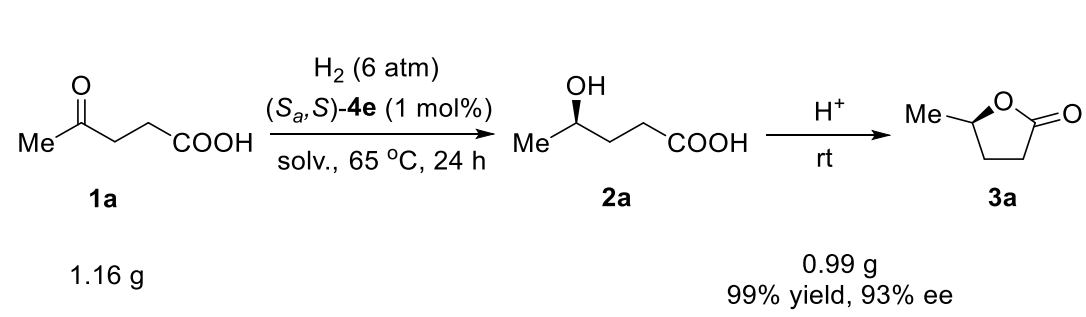

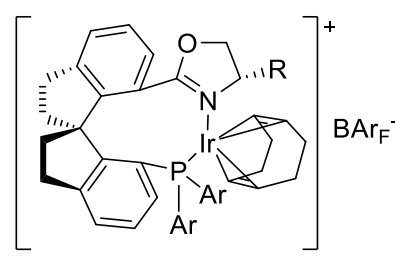

$\left(S_{a}, S\right)-4 e$

$\mathrm{Ar}=3,5-{ }^{-} \mathrm{Bu}_{2} \mathrm{C}_{6} \mathrm{H}_{3}, \mathrm{R}=\mathrm{CH}_{2}-1-\mathrm{Np}$

Figure S2. Gram-scale experiment for carboxyl-directed enantioselective hydrogenation of 4-oxopentanoic acid (1a). Reaction conditions: $10 \mathrm{mmol}$ scale, [substrate] $=0.5 \mathrm{~mol} / \mathrm{L},\left(S_{\mathrm{a}}, S\right)-4 \mathrm{e}(1 \mathrm{~mol} \%), \mathrm{H}_{2}(6 \mathrm{~atm}), \mathrm{Et}_{3} \mathrm{~N}(0.5$ equiv). Full conversions were obtained within $48 \mathrm{~h}$. The yield and ee value were measured after conversion to lactones.<smiles>[R]CCC(C)=O</smiles>

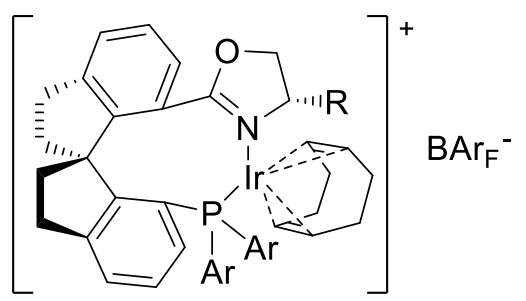

$$
\begin{gathered}
\left(S_{a}, S\right)-4 e \\
\mathrm{Ar}=3,5-{ }^{t} \mathrm{Bu}_{2} \mathrm{C}_{6} \mathrm{H}_{3}, \mathrm{R}=\mathrm{CH}_{2}-1-\mathrm{Np}
\end{gathered}
$$<smiles>C[C@H](O)CCC(=O)Nc1ccccc1</smiles>

$53 \%$ yield, $14 \%$ ee<smiles>C[C@H](O)CCNC(=O)Nc1ccccc1</smiles>

$42 \%$ yield, $27 \%$ ee<smiles>C[C@@H](O)CCC#N</smiles>

No conversion

Figure S3. Other directing groups for the hydrogenation reaction. Reaction conditions: $0.25 \mathrm{mmol}$ scale, [substrate] $=0.125 \mathrm{~mol} / \mathrm{L},\left(S_{\mathrm{a}}, S\right)-4 \mathbf{e}(2 \mathrm{~mol} \%), \mathrm{H}_{2}(6 \mathrm{~atm}), \mathrm{Et}_{3} \mathrm{~N}(0.5$ equiv). Full conversions were obtained within 24 h. ${ }^{\text {a }}$ 


\section{Preparation and Analytical Data of Iridium Catalysts}

All the ligands were prepared from the optically pure spiro phosphino-acids $(S)$-S1 according to our previously reported procedures. ${ }^{[1]}$

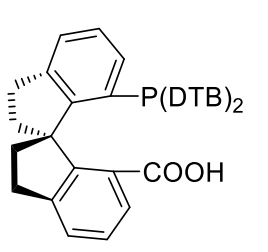

$\left(S_{\mathrm{a}}\right)-\mathrm{S} 1$

DTB $=3,5-\left({ }^{t} \mathrm{Bu}\right)_{2} \mathrm{C}_{6} \mathrm{H}_{3}$

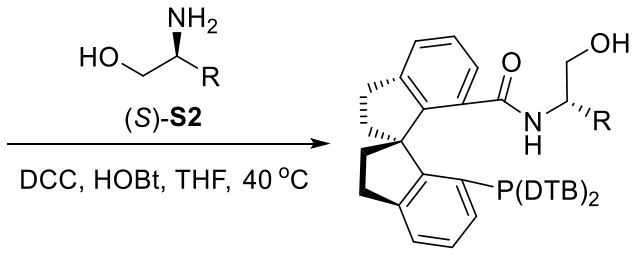

$\left(S_{a}, S\right)-S 3$

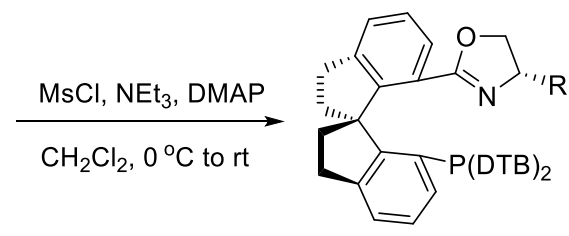

$\left(S_{\mathrm{a}}, S\right)-\mathbf{S 4}$

$\left(S_{\mathrm{a}, S}\right)-\mathrm{S} 4$

White solid, m.p.: $105-106{ }^{\circ} \mathrm{C} . \quad[\alpha]_{\mathrm{D}}{ }^{18}-140\left(c 0.5, \mathrm{CH}_{2} \mathrm{Cl}_{2}\right)$.

$\underline{{ }^{1} \mathrm{HMR}} \underline{\underline{\mathrm{N}}} \underline{(400} \underline{\mathrm{MHz}}, \underline{\left.\mathrm{CDCl}_{3}\right)}$

$\delta$ 8.03-7.95 (m, 1H), 7.86-7.77 (m, 1H), $7.69(\mathrm{~d}, J=8.2 \mathrm{~Hz}, 1 \mathrm{H}), 7.63(\mathrm{~d}, J=$ $5.4 \mathrm{~Hz}, 1 \mathrm{H}), 7.51-7.42(\mathrm{~m}, 2 \mathrm{H}), 7.37-7.27(\mathrm{~m}, 3 \mathrm{H}), 7.24(\mathrm{~d}, J=8.5 \mathrm{~Hz}, 2 \mathrm{H})$, $7.14(\mathrm{~d}, J=7.3 \mathrm{~Hz}, 1 \mathrm{H}), 7.07(\mathrm{~d}, J=7.1 \mathrm{~Hz}, 1 \mathrm{H}), 7.01(\mathrm{t}, J=7.4 \mathrm{~Hz}, 1 \mathrm{H})$, 6.98-6.90 (m, 3H), 6.81 (dd, $J=7.8,1.7 \mathrm{~Hz}, 2 \mathrm{H}), 4.40-4.27(\mathrm{~m}, 1 \mathrm{H}), 3.59$ (dd, $J=8.4,6.3 \mathrm{~Hz}, 1 \mathrm{H}), 3.36$ (t, $J=8.8 \mathrm{~Hz}, 1 \mathrm{H}), 3.21(\mathrm{~d}, J=4.4 \mathrm{~Hz}, 1 \mathrm{H}), 3.11-$ $2.84(\mathrm{~m}, 4 \mathrm{H}), 2.64(\mathrm{dd}, J=15.7,8.8 \mathrm{~Hz}, 1 \mathrm{H}), 2.31(\mathrm{dd}, J=14.4,9.9 \mathrm{~Hz}, 1 \mathrm{H})$, 2.25-2.17 (m, 1H), 2.00-1.88 (m, 1H), 1.86-1.74 (m, 1H), $1.17(\mathrm{~s}, 36 \mathrm{H})$.

${ }^{13} \mathrm{C} \underline{\mathrm{NMR}} \quad\left(101 \mathrm{MHz}, \underline{\left.\mathrm{CDCl}_{3}\right)}\right.$

$\delta 164.3,154.2,154.0,149.9,149.7,149.6,149.0,145.2,144.4,144.3,137.5$, $137.3,137.1,134.6,133.9,133.8,133.6,132.7,132.0,128.7,128.6,128.5$, $128.4,128.3,128.1,127.0,126.6,126.5,126.4,126.3,126.0,125.8,125.5$, $125.3,124.6,124.0,121.6,121.4,70.9,66.2,63.3,39.3,38.7,38.1,34.8,34.7$, $31.4,31.3,31.0,30.7$.

${ }^{31} \mathrm{P}$ NMR $\left(162 \mathrm{MHz}, \mathrm{CDCl}_{3}\right)$

$\delta-17.0(\mathrm{~s})$.

$\underline{\text { HRMS }} \underline{(\mathrm{ESI})}$

Calcd for $\left[\mathrm{C}_{59} \mathrm{H}_{68} \mathrm{NOP}, \mathrm{M}+\mathrm{Na}\right]^{+}:$860.4931, Found: 860.4937. 
$(S a, S)-4 \mathrm{e}$

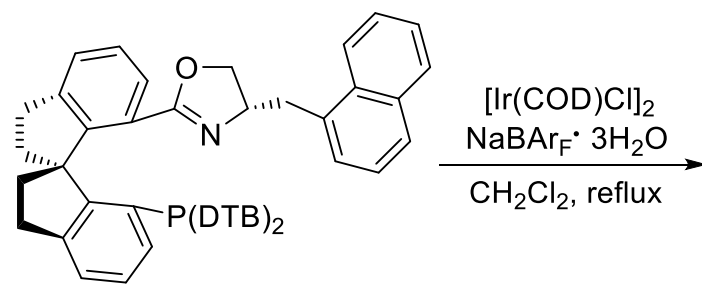

$\left(S_{a}, S\right)-S 4$

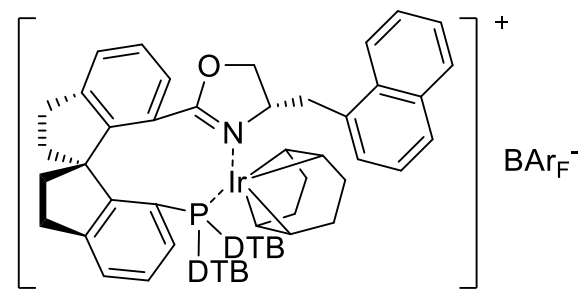

$\left(S_{a}, S\right)-4 e$

In a glove box, ligand $\left(S_{\mathrm{a}}, S\right)-\mathbf{S 4}(628.5 \mathrm{mg}, 0.75 \mathrm{mmol}),[\operatorname{Ir}(\mathrm{COD}) \mathrm{Cl}]_{2}(296 \mathrm{mg}, 0.44$ mmol) and $\mathrm{NaBAr}_{\mathrm{F}} \cdot 3 \mathrm{H}_{2} \mathrm{O}(882 \mathrm{mg}, 0.94 \mathrm{mmol})$ were added to $13 \mathrm{~mL}$ of $\mathrm{CH}_{2} \mathrm{Cl}_{2}$ in a 50 $\mathrm{mL}$ Schlenk tube under argon atmosphere. The mixture was heated at reflux for $30 \mathrm{~min}$. The TLC analysis revealed no free ligand existed. After cooling to room temperature, the mixture was concentrated under reduced pressure and the residue was purified by a flash column chromatography on silica gel column eluting with $\mathrm{CH}_{2} \mathrm{Cl}_{2}$ /petroleum ether $(2: 1$, $\mathrm{v} / \mathrm{v})$ to offer $\left(S_{\mathrm{a}}, S\right)-4 \mathrm{e}(1.22 \mathrm{~g}, 81 \%)$ as an orange-yellow solid. m.p.: $180-181{ }^{\circ} \mathrm{C} .[\alpha]_{\mathrm{D}}{ }^{18}$ $+106\left(c 0.5, \mathrm{CH}_{2} \mathrm{Cl}_{2}\right)$.

$\underline{{ }^{1} \mathrm{H}} \underline{\mathrm{NMR}} \quad \underline{(400} \underline{\mathrm{MHz}}, \underline{\left.\mathrm{CDCl}_{3}\right)}$

$\delta 8.25(\mathrm{dd}, J=6.3,2.0 \mathrm{~Hz}, 1 \mathrm{H}), 7.96-7.79(\mathrm{~m}, 4 \mathrm{H}), 7.78-7.65(\mathrm{~m}, 10 \mathrm{H}), 7.63-$ $7.47(\mathrm{~m}, 11 \mathrm{H}), 7.42(\mathrm{t}, J=7.7 \mathrm{~Hz}, 1 \mathrm{H}), 7.30(\mathrm{~d}, J=7.5 \mathrm{~Hz}, 1 \mathrm{H}), 7.23-7.13$ $(\mathrm{m}, 2 \mathrm{H}), 6.76(\mathrm{~d}, J=9.6 \mathrm{~Hz}, 1 \mathrm{H}), 6.17(\mathrm{~d}, J=13.4 \mathrm{~Hz}, 1 \mathrm{H}), 4.94(\mathrm{~d}, J=6.4$ $\mathrm{Hz}, 1 \mathrm{H}), 4.30-4.15(\mathrm{~m}, 2 \mathrm{H}), 4.11-3.92(\mathrm{~m}, 2 \mathrm{H}), 3.47(\mathrm{dd}, J=13.9,4.1 \mathrm{~Hz}$, $1 \mathrm{H}), 3.28-3.15(\mathrm{~m}, 2 \mathrm{H}), 2.98-2.86(\mathrm{~m}, 1 \mathrm{H}), 2.85-2.74(\mathrm{~m}, 2 \mathrm{H}), 2.70-2.54(\mathrm{~m}$, 2H), 2.33-2.19 (m, 2H), 2.16-2.02 (m, 3H), $1.27(\mathrm{~s}, 26 \mathrm{H}), 1.06(\mathrm{~s}, 12 \mathrm{H}), 0.95-$ $0.78(\mathrm{~m}, 2 \mathrm{H}), 0.50-0.24(\mathrm{~m}, 2 \mathrm{H})$.

${ }^{13} \mathrm{C} \underline{\mathrm{NMR}} \quad\left(101 \mathrm{MHz}_{2}, \mathrm{CDCl}_{3}\right)$

$\delta 173.4,162.4,161.9,161.5,161.0,148.2,147.7,147.6,145.8,144.0,134.8$, $134.1,132.5,132.2,132.1,132.0,131.6,130.7,130.2,129.5,129.0,128.8$, $128.7,128.7,128.6,128.0,127.9,127.7,127.2,126.9,126.5,126.4,125.9$, $125.6,125.4,123.9,123.2,122.2,120.5,117.4,75.0,73.4,70.9,68.0,67.2$, 66.9, 66.0, 62.7, 41.3, 37.1, 35.0, 33.7, 31.3, 31.2, 31.1, 30.9, 30.7, 30.4, 30.3, 29.1, 26.6.

${ }^{31} \mathrm{P}$ NMR $\left(162 \mathrm{MHz}, \mathrm{CDCl}_{3}\right)$

$\delta 16.5(\mathrm{~s})$.

$\underline{\text { HRMS }} \underline{(E S I)}$

Calcd for $\left[\mathrm{C}_{67} \mathrm{H}_{80} \mathrm{IrNOP}, \mathrm{M}\right]^{+}:$1138.5601, Found: 1138.5591. 


\section{Preparation and Analytical Data of Substrates}

All the substrates are prepared according to the literature or purchased and used directly.

4-oxohexanoic acid (1b) $)^{[2]}$

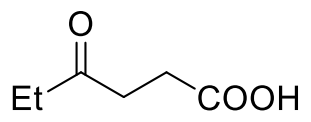

${ }^{1} \mathrm{H}$ NMR (400 MHz, $\left.\mathrm{CDCl}_{3}\right) \delta 10.35$ (brs, $\left.1 \mathrm{H}\right), 2.71(\mathrm{t}, J=6.4 \mathrm{~Hz}, 2 \mathrm{H}), 2.62(\mathrm{t}, J=6.4 \mathrm{~Hz}$, $2 \mathrm{H}), 2.46$ (q, $J=7.2 \mathrm{~Hz}, 2 \mathrm{H}), 1.05(\mathrm{t}, J=7.2 \mathrm{~Hz}, 3 \mathrm{H})$.

4-oxooctanoic acid (1c) $)^{[2]}$<smiles>CCCC(=O)CCC(=O)O</smiles>

${ }^{1} \mathrm{H}$ NMR (400 MHz, $\left.\mathrm{CDCl}_{3}\right) \delta 11.65(\mathrm{brs}, 1 \mathrm{H}), 2.67(\mathrm{t}, J=6.4 \mathrm{~Hz}, 2 \mathrm{H}), 2.57(\mathrm{t}, J=6.5 \mathrm{~Hz}$, $2 \mathrm{H}), 2.40(\mathrm{t}, J=7.5 \mathrm{~Hz}, 2 \mathrm{H}), 1.60-1.44(\mathrm{~m}, 2 \mathrm{H}), 1.43-1.18(\mathrm{~m}, 2 \mathrm{H}), 0.85(\mathrm{t}, J=7.3 \mathrm{~Hz}$, $3 \mathrm{H})$.

4-oxoundecanoic acid (1d) $)^{[2]}$

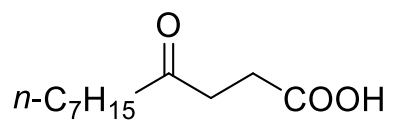

${ }^{1} \mathrm{H} \mathrm{NMR}\left(400 \mathrm{MHz}, \mathrm{CDCl}_{3}\right) \delta 11.38$ (brs, $\left.1 \mathrm{H}\right), 2.70(\mathrm{t}, J=6.4 \mathrm{~Hz}, 2 \mathrm{H}), 2.61(\mathrm{t}, J=6.3 \mathrm{~Hz}$, $2 \mathrm{H}), 2.43(\mathrm{t}, J=7.5 \mathrm{~Hz}, 2 \mathrm{H}), 1.65-1.48(\mathrm{~m}, 2 \mathrm{H}), 1.26(\mathrm{~s}, 8 \mathrm{H}), 0.86(\mathrm{t}, J=6.6 \mathrm{~Hz}, 3 \mathrm{H})$

6-methyl-4-oxoheptanoic acid (1e $)^{[3]}$

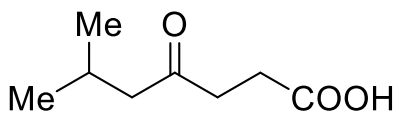

${ }^{1} \mathrm{H}$ NMR $\left(400 \mathrm{MHz}, \mathrm{CDCl}_{3}\right) \delta 2.70(\mathrm{t}, J=6.1 \mathrm{~Hz}, 2 \mathrm{H}), 2.63(\mathrm{t}, J=6.7 \mathrm{~Hz}, 2 \mathrm{H}), 2.33(\mathrm{~d}, J$ $=7.0 \mathrm{~Hz}, 2 \mathrm{H}), 2.23-2.09(\mathrm{~m}, 1 \mathrm{H}), 0.92(\mathrm{~d}, J=6.6 \mathrm{~Hz}, 6 \mathrm{H})$.

5-methyl-4-oxohexanoic acid (1f) ${ }^{[4]}$<smiles>CC(C)C(=O)CCC(=O)O</smiles>

${ }^{1} \mathrm{H}$ NMR (400 MHz, $\left.\mathrm{CDCl}_{3}\right) \delta 11.72$ (brs, 1H), 2.74 (m, 2H), 2.66-2.54 (m, 3H), 1.08 (t, J $=6.4 \mathrm{~Hz}, 6 \mathrm{H})$.

4-cyclopentyl-4-oxobutanoic acid (1g) $)^{[5]}$<smiles>O=C(O)CCC(=O)C1CCCC1</smiles> 
${ }^{1} \mathrm{H}$ NMR $\left(400 \mathrm{MHz}, \mathrm{CDCl}_{3}\right) \delta 2.93(\mathrm{p}, J=8.0 \mathrm{~Hz}, 1 \mathrm{H}), 2.80(\mathrm{t}, J=6.5 \mathrm{~Hz}, 2 \mathrm{H}), 2.66(\mathrm{t}, J$ $=6.5 \mathrm{~Hz}, 2 \mathrm{H}), 1.92-1.74(\mathrm{~m}, 4 \mathrm{H}), 1.73-1.54(\mathrm{~m}, 4 \mathrm{H})$.

4-cyclohexyl-4-oxobutanoic acid (1h) ${ }^{[6]}$

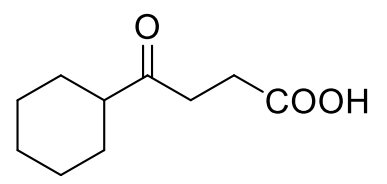

${ }^{1} \mathrm{H}$ NMR (400 MHz, $\left.\mathrm{CDCl}_{3}\right) \delta 11.29$ (brs s, 1H), $2.73(\mathrm{t}, J=6.5 \mathrm{~Hz}, 2 \mathrm{H}), 2.59(\mathrm{t}, J=6.5$ $\mathrm{Hz}, 2 \mathrm{H}), 2.35$ (m, 1H), 1.90-1.80 (m, 2H), 1.75 (m, 2H), 1.64 (m, 1H), 1.41-1.09 (m, 5H).

\section{4-oxo-5-phenylpentanoic acid (1i) $)^{[7]}$}

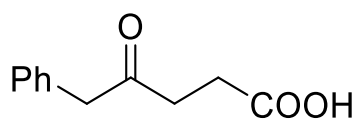

${ }^{1} \mathrm{H}$ NMR (400 MHz, $\left.\mathrm{CDCl}_{3}\right) \delta 11.50(\mathrm{~s}, 1 \mathrm{H}), 7.37(\mathrm{t}, J=7.3 \mathrm{~Hz}, 2 \mathrm{H}), 7.34-7.28(\mathrm{~m}, 1 \mathrm{H})$, $7.24(\mathrm{~d}, J=7.1 \mathrm{~Hz}, 2 \mathrm{H}), 3.77(\mathrm{~s}, 2 \mathrm{H}), 2.78(\mathrm{t}, J=6.4 \mathrm{~Hz}, 2 \mathrm{H}), 2.64(\mathrm{t}, J=6.4 \mathrm{~Hz}, 2 \mathrm{H})$.

\section{4-oxo-6-phenylhexanoic acid $(1 \mathbf{j})^{[8]}$}

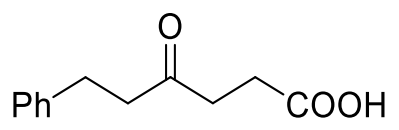

${ }^{1} \mathrm{H}$ NMR $\left(400 \mathrm{MHz}, \mathrm{CDCl}_{3}\right) \delta 11.31(\mathrm{~s}, 1 \mathrm{H}), 7.28(\mathrm{t}, J=7.3 \mathrm{~Hz}, 2 \mathrm{H}), 7.19(\mathrm{t}, J=8.1 \mathrm{~Hz}$, $3 \mathrm{H}), 2.91(\mathrm{t}, J=7.6 \mathrm{~Hz}, 2 \mathrm{H}), 2.78(\mathrm{t}, J=7.5 \mathrm{~Hz}, 2 \mathrm{H}), 2.73-2.66(\mathrm{~m}, 2 \mathrm{H}), 2.66-2.60(\mathrm{~m}$, $2 \mathrm{H})$.

6-(benzyloxy)-4-oxohexanoic acid (1k) ${ }^{[9]}$<smiles>O=C(O)CCC(=O)CCOCc1ccccc1</smiles>

${ }^{1} \mathrm{H}$ NMR $\left(400 \mathrm{MHz}, \mathrm{CDCl}_{3}\right) \delta 7.38-7.27(\mathrm{~m}, 5 \mathrm{H}), 4.50(\mathrm{~s}, 2 \mathrm{H}), 3.75(\mathrm{t}, J=6.2 \mathrm{~Hz}, 2 \mathrm{H})$, $2.76(\mathrm{dt}, J=12.2$ and $6.3 \mathrm{~Hz}, 4 \mathrm{H}), 2.64(\mathrm{t}, J=6.4 \mathrm{~Hz}, 2 \mathrm{H})$.

8-(benzyloxy)-4-oxooctanoic acid (11) ${ }^{[10]}$<smiles>O=C(O)CCC(=O)CCCCOCc1ccccc1</smiles>

${ }^{1} \mathrm{H}$ NMR (400 MHz, $\left.\mathrm{CDCl}_{3}\right) \delta$ 7.37-7.24 (m, 5H), $4.49(\mathrm{~s}, 2 \mathrm{H}), 3.47(\mathrm{t}, J=6.1 \mathrm{~Hz}, 2 \mathrm{H})$, 2.73-2.65 (m, 2H), $2.61(\mathrm{t}, J=6.5 \mathrm{~Hz}, 2 \mathrm{H}), 2.47(\mathrm{t}, J=7.1 \mathrm{~Hz}, 2 \mathrm{H}), 1.77-1.55(\mathrm{~m}, 4 \mathrm{H})$. ${ }^{13} \mathrm{C}$ NMR $\left(100 \mathrm{MHz}, \mathrm{CDCl}_{3}\right) \delta 208.7,178.4$, 138.5, 128.4, 127.7, 127.6, 72.9, 69.9, 42.3, 36.8, 29.1, 27.7, 20.5. HRMS (ESI) Calcd for $\left[\mathrm{C}_{15} \mathrm{H}_{20} \mathrm{O}_{4}, \mathrm{M}-\mathrm{H}\right]^{-}: 263.1289$, Found: 263.1294. 


\section{8-methyl-4-oxonon-7-enoic acid (1m) ${ }^{[11]}$}

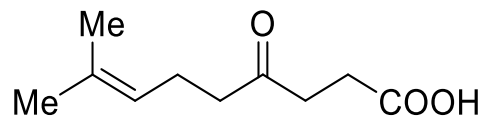

${ }^{1} \mathrm{H}$ NMR $\left(400 \mathrm{MHz}, \mathrm{CDCl}_{3}\right) \delta 11.23(\mathrm{~s}, 1 \mathrm{H}), 5.04(\mathrm{~m}, 1 \mathrm{H}), 2.70(\mathrm{t}, J=6.5 \mathrm{~Hz}, 2 \mathrm{H}), 2.62$ $(\mathrm{t}, J=6.3 \mathrm{~Hz}, 2 \mathrm{H}), 2.46(\mathrm{t}, J=7.4 \mathrm{~Hz}, 2 \mathrm{H}), 2.26(\mathrm{q}, J=7.3 \mathrm{~Hz}, 2 \mathrm{H}), 1.66(\mathrm{~s}, 3 \mathrm{H}), 1.60(\mathrm{~s}$, $3 \mathrm{H})$.

4-oxooct-7-enoic acid (1n) ${ }^{[12]}$

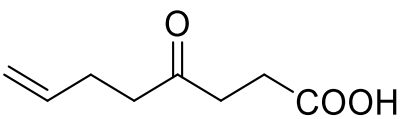

${ }^{1} \mathrm{H}$ NMR (400 MHz, $\left.\mathrm{CDCl}_{3}\right) \delta 5.80$ (ddt, $J=16.8$ and 10.2 and $6.5 \mathrm{~Hz}, 1 \mathrm{H}$ ), 5.01 (ddd, $J=$ 13.7 and 11.5 and $1.4 \mathrm{~Hz}, 2 \mathrm{H}), 2.72(\mathrm{t}, J=6.2 \mathrm{~Hz}, 2 \mathrm{H}), 2.64(\mathrm{t}, J=6.1 \mathrm{~Hz}, 2 \mathrm{H}), 2.56(\mathrm{t}, J$ $=7.4 \mathrm{~Hz}, 2 \mathrm{H}), 2.34(\mathrm{dd}, J=13.9$ and $7.2 \mathrm{~Hz}, 2 \mathrm{H})$.

7-ethoxy-4,7-dioxoheptanoic acid (10) $)^{[13]}$<smiles>CCOC(=O)CCC(=O)CCC(=O)O</smiles>

${ }^{1} \mathrm{H}$ NMR (400 MHz, $\left.\mathrm{CDCl}_{3}\right) \delta 4.12(\mathrm{q}, J=7.1 \mathrm{~Hz}, 2 \mathrm{H}), 2.77(\mathrm{dd}, J=12.0$ and $5.9 \mathrm{~Hz}, 4 \mathrm{H})$, $2.65(\mathrm{t}, J=6.5 \mathrm{~Hz}, 2 \mathrm{H}), 2.60(\mathrm{t}, J=6.6 \mathrm{~Hz}, 2 \mathrm{H}), 1.24(\mathrm{t}, J=7.1 \mathrm{~Hz}, 3 \mathrm{H})$.

\section{General Procedure and Analytical Data of Hydrogenation}

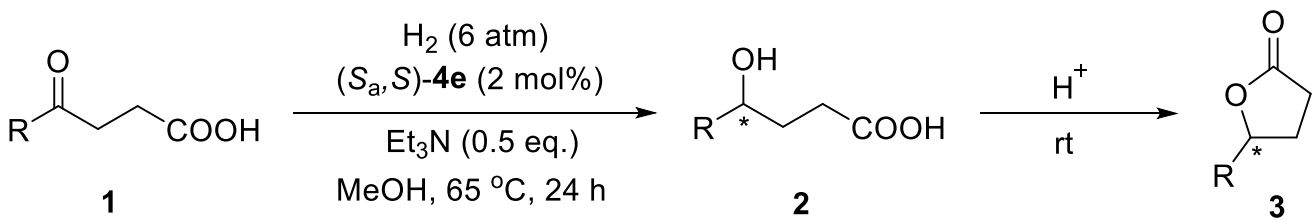

To a hydrogenation tube was charged with a stir bar, substrate $1(0.25 \mathrm{mmol})$, catalyst $\left(S_{a}, S\right)-4 \mathrm{e}(10.0 \mathrm{mg}, 0.005 \mathrm{mmol}), \mathrm{NEt}_{3}(12.5 \mathrm{mg}, 0.125 \mathrm{mmol})$ and $\mathrm{MeOH}(2 \mathrm{~mL})$. The hydrogenation tube was put into an autoclave. The air in the autoclave was replaced with hydrogen for five times. The autoclave was then charged with hydrogen to $6 \mathrm{~atm}$, and the reaction mixture was stirred at $65{ }^{\circ} \mathrm{C}$ for $24 \mathrm{~h}$. After releasing hydrogen, the solution was treated with $5 \% \mathrm{NaOH}(0.5 \mathrm{~mL})$. The aqueous layer was washed with $\mathrm{Et}_{2} \mathrm{O}(8 \mathrm{~mL})$, acidified with $1 \mathrm{~N} \mathrm{HCl}$ ( $\mathrm{pH} 5 \sim 6$ ), reacted for $30 \mathrm{~min}$ (the reaction time was different between substrates), and then extracted with $\mathrm{Et}_{2} \mathrm{O}(3 \times 8 \mathrm{~mL})$. The combined extracts were washed with a saturated solution of $\mathrm{NaCl}$, dried over $\mathrm{MgSO}_{4}$, and evaporated in vacuo to give the product 3 . The product was analyzed on GC or HPLC to determine $e e$ value. 
(R)-5-methyldihydrofuran-2(3H)-one (3a) $)^{[14]}$

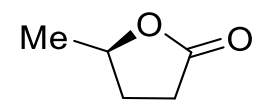

Colorless oil, $91 \%$ yield, $93 \%$ ee, $[\alpha]_{\mathrm{D}}{ }^{20}+29.1$ ( $c 1.75$, chloroform), GC (Supelco $\beta$-DEX ${ }^{\mathrm{TM}}$ $225, \mathrm{df}=0.25 \mu \mathrm{m}, 0.25 \mathrm{~mm}$ i.d. $\times 25 \mathrm{~m}$ ) using a flame ionization detector (FID) operating at $250{ }^{\circ} \mathrm{C}$. Injector temperature was $230^{\circ} \mathrm{C}$. The carrier gas was nitrogen with a flow rate of $1.0 \mathrm{~mL} / \mathrm{min}$. The following temperature program was used in the analysis: $100{ }^{\circ} \mathrm{C}(8$ $\mathrm{min})-5^{\circ} \mathrm{C} / \mathrm{min}-200{ }^{\circ} \mathrm{C}(10 \mathrm{~min}), t_{\mathrm{R}}=21.70 \mathrm{~min}$ for major isomer and $t_{\mathrm{R}}=22.84 \mathrm{~min}$ for minor isomer. The absolute configuration of $(R)$-3a was determined by comparing with optical rotation reported in literature ${ }^{14}\left\{[\alpha]_{\mathrm{D}}^{20}+31.0\right.$ (c 1.5, chloroform) $\}$.

$\underline{{ }^{1} \mathrm{H}} \underline{\mathrm{NMR}} \quad \underline{(400} \mathrm{MHz}, \underline{\left.\mathrm{CDCl}_{3}\right)}$

$\delta$ 4.65-4.60 (m, 1H), 2.55-2.51 (m, 2H), 2.39-2.30 (m, 1H), 1.86-1.77 (m, $1 \mathrm{H}), 1.39(\mathrm{~d}, J=6.0 \mathrm{~Hz}, 3 \mathrm{H})$.

\section{$(R)$-5-ethyldihydrofuran-2(3H)-one (3b $)^{[15]}$}

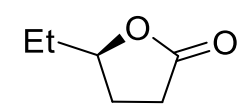

Colorless oil, 92\% yield, 95\% ee, $[\alpha]_{\mathrm{D}}{ }^{20}+47.6$ (c 1.0, methanol), GC(Supelco $\beta$-DEX ${ }^{\mathrm{TM}}$ $225, \mathrm{df}=0.25 \mu \mathrm{m}, 0.25 \mathrm{~mm}$ i.d. $\times 25 \mathrm{~m}$ ) using a flame ionization detector (FID) operating at $250{ }^{\circ} \mathrm{C}$. Injector temperature was $230^{\circ} \mathrm{C}$. The carrier gas was nitrogen with a flow rate of $1.0 \mathrm{~mL} / \mathrm{min}$. The following temperature program was used in the analysis: $100{ }^{\circ} \mathrm{C}$ $5{ }^{\circ} \mathrm{C} / \mathrm{min}-200{ }^{\circ} \mathrm{C}(10 \mathrm{~min}), t_{\mathrm{R}}=12.12 \mathrm{~min}$ for major isomer and $t_{\mathrm{R}}=12.83 \mathrm{~min}$ for minor isomer. The absolute configuration of $(R)-3 \mathbf{b}$ was determined by comparing with optical rotation reported in literature ${ }^{15}\left\{[\alpha]_{\mathrm{D}}{ }^{20}+50.0\right.$ (c 1.0, methanol) $\}$.

${ }^{1} \mathrm{H} \underline{\mathrm{NMR}} \quad \underline{(400} \mathrm{MHz}, \underline{\left.\mathrm{CDCl}_{3}\right)}$

$\delta$ 4.45-4.38 (m, 1H), 2.53-2.49 (m, 2H), 2.34-2.28 (m, 1H), 1.89-1.59 (m, $3 \mathrm{H}), 0.98(\mathrm{t}, J=7.2 \mathrm{~Hz}, 3 \mathrm{H})$.

\section{(R)-5-butyldihydrofuran-2(3H)-one $(3 \mathrm{c})^{[16]}$}

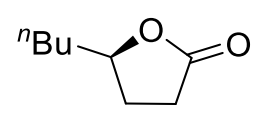

Colorless oil, 96\% yield, 94\% ee, $[\alpha]_{\mathrm{D}}{ }^{20}+39.8$ ( $c$ 1.0, methanol), GC(Supelco $\beta$-DEX ${ }^{\mathrm{TM}}$ $225, \mathrm{df}=0.25 \mu \mathrm{m}, 0.25 \mathrm{~mm}$ i.d. $\times 25 \mathrm{~m}$ ) using a flame ionization detector (FID) operating at $250{ }^{\circ} \mathrm{C}$. Injector temperature was $230^{\circ} \mathrm{C}$. The carrier gas was nitrogen with a flow rate of $1.0 \mathrm{~mL} / \mathrm{min}$. The following temperature program was used in the analysis: $100{ }^{\circ} \mathrm{C}$ $5{ }^{\circ} \mathrm{C} / \mathrm{min}-200{ }^{\circ} \mathrm{C}(10 \mathrm{~min}), t_{\mathrm{R}}=14.36 \mathrm{~min}$ for major isomer and $t_{\mathrm{R}}=14.64 \mathrm{~min}$ for minor isomer. The absolute configuration of $(R)-3 \mathbf{c}$ was determined by comparing with optical rotation reported in literature ${ }^{16}\left\{[\alpha]_{\mathrm{D}}{ }^{25}-45.3\right.$ (c 1.0, methanol) $\}$.

$\underline{{ }^{1} \mathrm{H}} \underline{\mathrm{NMR}} \quad \underline{(400} \underline{\mathrm{MHz}}, \underline{\left.\mathrm{CDCl}_{3}\right)}$

$\delta 4.50-4.43(\mathrm{~m}, 1 \mathrm{H}), 2.51(\mathrm{dd}, J=9.4$ and $7.0 \mathrm{~Hz}, 2 \mathrm{H}), 2.30(\mathrm{dq}, J=13.5$ and $6.7 \mathrm{~Hz}, 1 \mathrm{H}), 1.88-1.79(\mathrm{~m}, 1 \mathrm{H}), 1.75-1.68(\mathrm{~m}, 1 \mathrm{H}), 1.61-1.55(\mathrm{~m}, 1 \mathrm{H}), 1.45-$ $1.31(\mathrm{~m}, 4 \mathrm{H}), 0.90(\mathrm{t}, J=7.1 \mathrm{~Hz}, 3 \mathrm{H})$. 


\section{(R)-5-heptyldihydrofuran-2(3H)-one $(3 \mathrm{~d})^{[17]}$}

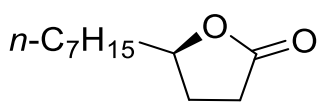

Colorless oil, 98\% yield, 93\% ee, $[\alpha]_{\mathrm{D}}{ }^{20}+35.2$ (c 1.0, chloroform), GC(Supelco $\beta$-DEX ${ }^{\mathrm{TM}}$ $225, \mathrm{df}=0.25 \mu \mathrm{m}, 0.25 \mathrm{~mm}$ i.d. $\times 25 \mathrm{~m}$ ) using a flame ionization detector (FID) operating at $250{ }^{\circ} \mathrm{C}$. Injector temperature was $230{ }^{\circ} \mathrm{C}$. The carrier gas was nitrogen with a flow rate of $1.0 \mathrm{~mL} / \mathrm{min}$. The following temperature program was used in the analysis: $100{ }^{\circ} \mathrm{C}$ $5{ }^{\circ} \mathrm{C} / \mathrm{min}-180{ }^{\circ} \mathrm{C}-0.5{ }^{\circ} \mathrm{C} / \mathrm{min}-200{ }^{\circ} \mathrm{C}(5 \mathrm{~min}), t_{\mathrm{R}}=21.05 \mathrm{~min}$ for major isomer and $t_{\mathrm{R}}=$ $21.31 \mathrm{~min}$ for minor isomer. The absolute configuration of $(R)-\mathbf{3 d}$ was determined by comparing with optical rotation reported in literature ${ }^{[18]}\left\{[\alpha]_{\mathrm{D}}{ }^{20}+38.7(c 0.8\right.$, methanol $\left.)\right\}$. $\underline{{ }^{1} \mathrm{H}} \underline{\mathrm{NMR}} \quad \underline{(400} \underline{\mathrm{MHz}}, \underline{\left.\mathrm{CDCl}_{3}\right)}$

$\delta$ 4.49-4.42 (m, 1H), $2.49(\mathrm{dd}, J=9.5$ and $6.9 \mathrm{~Hz}, 2 \mathrm{H}), 2.39-2.25(\mathrm{~m}, 1 \mathrm{H})$, $1.87-1.77(\mathrm{~m}, 1 \mathrm{H}), 1.73-1.66(\mathrm{~m}, 1 \mathrm{H}), 1.59-1.52(\mathrm{~m}, 1 \mathrm{H}), 1.44-1.24(\mathrm{~m}$, $10 \mathrm{H}), 0.84(\mathrm{t}, J=6.6 \mathrm{~Hz}, 3 \mathrm{H})$.

(+)-5-isobutyldihydrofuran-2(3H)-one $(3 \mathrm{e})^{[19]}$

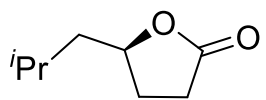

Colorless oil, $94 \%$ yield, $95 \%$ ee, $[\alpha]_{\mathrm{D}}{ }^{20}+30.6$ ( $c 0.5$, chloroform), GC(Supelco $\beta$-DEX ${ }^{\mathrm{TM}}$ $225, \mathrm{df}=0.25 \mu \mathrm{m}, 0.25 \mathrm{~mm}$ i.d. $\times 25 \mathrm{~m}$ ) using a flame ionization detector (FID) operating at $250{ }^{\circ} \mathrm{C}$. Injector temperature was $230{ }^{\circ} \mathrm{C}$. The carrier gas was nitrogen with a flow rate of $1.0 \mathrm{~mL} / \mathrm{min}$. The following temperature program was used in the analysis: $100{ }^{\circ} \mathrm{C}$ $0.5{ }^{\circ} \mathrm{C} / \mathrm{min}-130{ }^{\circ} \mathrm{C}(10 \mathrm{~min}), t_{\mathrm{R}}=31.00 \mathrm{~min}$ for major isomer and $t_{\mathrm{R}}=31.58 \mathrm{~min}$ for minor isomer.

$\underline{{ }^{1} \mathrm{H}} \underline{\mathrm{NMR}} \quad \underline{(400} \underline{\mathrm{MHz}}, \underline{\left.\mathrm{CDCl}_{3}\right)}$

$\delta 4.57(\mathrm{tdd}, J=8.4,6.4,4.8 \mathrm{~Hz}, 1 \mathrm{H}), 2.53(\mathrm{dd}, J=9.6,6.7 \mathrm{~Hz}, 2 \mathrm{H}), 2.33(\mathrm{dq}$, $J=13.2,6.6 \mathrm{~Hz}, 1 \mathrm{H}), 1.94-1.75(\mathrm{~m}, 2 \mathrm{H}), 1.68(\mathrm{ddd}, J=14.5,8.6,6.1 \mathrm{~Hz}, 1 \mathrm{H})$, $1.40(\mathrm{ddd}, J=13.9,7.9,4.8 \mathrm{~Hz}, 1 \mathrm{H}), 0.95(\mathrm{dd}, J=6.7,4.0 \mathrm{~Hz}, 6 \mathrm{H})$.

\section{(S)-5-isopropyldihydrofuran-2(3H)-one $(3 f)^{[20]}$}

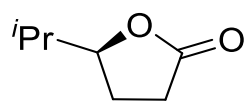

Colorless oil, $91 \%$ yield, $>99 \%$ ee, $[\alpha]_{\mathrm{D}}{ }^{20}+35.7$ ( $c$ 1.1, chloroform), GC(Supelco $\beta$-DEX ${ }^{\mathrm{TM}}$ $225, \mathrm{df}=0.25 \mu \mathrm{m}, 0.25 \mathrm{~mm}$ i.d. $\times 25 \mathrm{~m}$ ) using a flame ionization detector (FID) operating at $250{ }^{\circ} \mathrm{C}$. Injector temperature was $230{ }^{\circ} \mathrm{C}$. The carrier gas was nitrogen with a flow rate of $1.0 \mathrm{~mL} / \mathrm{min}$. The following temperature program was used in the analysis: $100{ }^{\circ} \mathrm{C}$ $5{ }^{\circ} \mathrm{C} / \mathrm{min}-200{ }^{\circ} \mathrm{C}(10 \mathrm{~min}), t_{\mathrm{R}}=11.19 \mathrm{~min}$ for major isomer and $t_{\mathrm{R}}=11.49 \mathrm{~min}$ for minor isomer. The absolute configuration of $(S)$-3f was determined by comparing with optical rotation reported in literature ${ }^{20}\left\{[\alpha]_{\mathrm{D}}^{20}+34.7\right.$ (c 1.1, chloroform) $\}$.

$\left.{ }^{1} \mathrm{H} \underline{\mathrm{NMR}} \quad \underline{(400} \underline{\mathrm{MHz}}, \underline{\mathrm{CDCl}_{3}}\right)$

$\delta$ 4.21-4.15 (m, 1H), 2.54-2.49 (m, 2H), 2.28-2.20 (m, 1H), 1.94-1.78 (m, $2 \mathrm{H}), 1.00(\mathrm{~d}, J=6.7 \mathrm{~Hz}, 3 \mathrm{H}), 0.92(\mathrm{~d}, J=6.8 \mathrm{~Hz}, 3 \mathrm{H})$. 
(+)-5-cyclopentyldihydrofuran-2(3H)-one $(3 \mathrm{~g})^{[21]}$

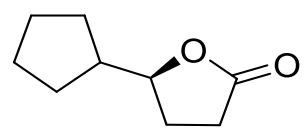

Colorless oil, $93 \%$ yield, $94 \%$ ee, $[\alpha]_{\mathrm{D}}{ }^{20}+34.2$ (c 1.0, chloroform), GC(Supelco $\beta$-DEX ${ }^{\mathrm{TM}}$ $225, \mathrm{df}=0.25 \mu \mathrm{m}, 0.25 \mathrm{~mm}$ i.d. $\times 25 \mathrm{~m}$ ) using a flame ionization detector (FID) operating at $250{ }^{\circ} \mathrm{C}$. Injector temperature was $230{ }^{\circ} \mathrm{C}$. The carrier gas was nitrogen with a flow rate of $1.0 \mathrm{~mL} / \mathrm{min}$. The following temperature program was used in the analysis: $100{ }^{\circ} \mathrm{C}$ $5{ }^{\circ} \mathrm{C} / \mathrm{min}-170{ }^{\circ} \mathrm{C}-1{ }^{\circ} \mathrm{C} / \mathrm{min}-200{ }^{\circ} \mathrm{C}, t_{\mathrm{R}}=19.48 \mathrm{~min}$ for major isomer and $t_{\mathrm{R}}=19.68 \mathrm{~min}$ for minor isomer.

$\underline{{ }^{1} \mathrm{H}} \underline{\mathrm{NMR}} \quad \underline{(400} \underline{\mathrm{MHz}}, \underline{\left.\mathrm{CDCl}_{3}\right)}$

$\delta 4.24(\mathrm{td}, J=8.2,6.5 \mathrm{~Hz}, 1 \mathrm{H}), 2.46(\mathrm{dd}, J=9.7,6.8 \mathrm{~Hz}, 2 \mathrm{H}), 2.23(\mathrm{dq}, J=$ $13.4,6.7 \mathrm{~Hz}, 1 \mathrm{H}), 1.99(\mathrm{~h}, J=8.1 \mathrm{~Hz}, 1 \mathrm{H}), 1.90-1.74(\mathrm{~m}, 2 \mathrm{H}), 1.74-1.63(\mathrm{~m}$, $1 \mathrm{H}), 1.63-1.45(\mathrm{~m}, 4 \mathrm{H}), 1.42-1.29(\mathrm{~m}, 1 \mathrm{H}), 1.25-1.11(\mathrm{~m}, 1 \mathrm{H})$.

\section{(S)-5-cyclohexyldihydrofuran-2(3H)-one $(3 h)^{[22]}$}

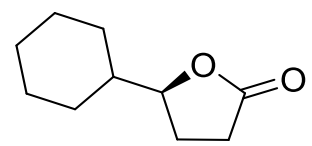

Colorless oil, $93 \%$ yield, $96 \%$ ee, $[\alpha]_{\mathrm{D}}{ }^{20}+32.2$ (c 1.0, methanol), GC(Supelco $\beta$-DEX ${ }^{\mathrm{TM}}$ $225, \mathrm{df}=0.25 \mu \mathrm{m}, 0.25 \mathrm{~mm}$ i.d. $\times 25 \mathrm{~m}$ ) using a flame ionization detector (FID) operating at $250{ }^{\circ} \mathrm{C}$. Injector temperature was set at $230{ }^{\circ} \mathrm{C}$. The carrier gas was nitrogen with a flow rate of $1.0 \mathrm{~mL} / \mathrm{min}$. The following temperature program was used in the analysis: $100{ }^{\circ} \mathrm{C}$ $5{ }^{\circ} \mathrm{C} / \mathrm{min}-150{ }^{\circ} \mathrm{C}-1{ }^{\circ} \mathrm{C} / \mathrm{min}-180{ }^{\circ} \mathrm{C}, t_{\mathrm{R}}=21.80 \mathrm{~min}$ for major isomer and $t_{\mathrm{R}}=22.12$ min for minor isomer. The absolute configuration of $(S)$-3h was determined by comparing with optical rotation reported in literature ${ }^{22}\left\{[\alpha]_{\mathrm{D}}{ }^{20}+29.8(c 0.01\right.$, methanol $\left.)\right\}$.

$\left.\underline{{ }^{1} \mathrm{H}} \underline{\mathrm{NMR}} \quad \underline{(400} \underline{\mathrm{MHz}}, \underline{\mathrm{CDCl}_{3}}\right)$

$\delta 4.18(\mathrm{dd}, J=15.1$ and $7.5 \mathrm{~Hz}, 1 \mathrm{H}), 2.54-2.41(\mathrm{~m}, 2 \mathrm{H}), 2.21(\mathrm{td}, J=13.0$ and $6.6 \mathrm{~Hz}, 1 \mathrm{H}), 1.96-1.85(\mathrm{~m}, 2 \mathrm{H}), 1.78-1.68(\mathrm{~m}, 2 \mathrm{H}), 1.68-1.57(\mathrm{~m}, 2 \mathrm{H}), 1.55-$ $1.43(\mathrm{~m}, 1 \mathrm{H}), 1.29-1.10(\mathrm{~m}, 3 \mathrm{H}), 1.05-0.90(\mathrm{~m}, 2 \mathrm{H})$.

(S)-5-benzyldihydrofuran-2(3H)-one $(3 i)^{[23]}$

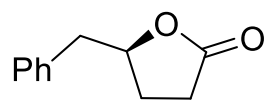

Colorless oil, $96 \%$ yield, $91 \%$ ee, $[\alpha]_{\mathrm{D}}{ }^{20}+20.2$ (c 1.0, chloroform), HPLC condition for corresponding amide: Chiralcel OD-H column $(25 \mathrm{~cm} \times 0.46 \mathrm{~cm}$ ID), $n$-hexane/2-propanol $=90: 10,1.0 \mathrm{~mL} / \mathrm{min}, 210 \mathrm{~nm}$ UV detector, $t_{\mathrm{R}}=10.62 \mathrm{~min}(S)$ and $t_{\mathrm{R}}=12.30 \mathrm{~min}(R)$. The absolute configuration of $(S)$-3i was determined by comparing with optical rotation reported in literature ${ }^{23}\left\{[\alpha]_{\mathrm{D}}{ }^{25}+24.7\right.$ (c 1.0, chloroform) $\}$.

${ }^{1} \mathrm{H} \underline{\mathrm{NMR}} \quad \underline{(400} \underline{\mathrm{MHz}}, \underline{\left.\mathrm{CDCl}_{3}\right)}$

$\delta 7.35-7.19(\mathrm{~m}, 5 \mathrm{H}), 4.72(\mathrm{~d}, J=6.5 \mathrm{~Hz}, 1 \mathrm{H}), 3.06(\mathrm{dd}, J=14.0$ and $6.1 \mathrm{~Hz}$, $1 \mathrm{H}), 2.92(\mathrm{dd}, J=14.0$ and $6.1 \mathrm{~Hz}, 1 \mathrm{H}), 2.53-2.30(\mathrm{~m}, 2 \mathrm{H}), 2.24(\mathrm{ddd}, J=$ 21.2 and 10.2 and $5.7 \mathrm{~Hz}, 1 \mathrm{H}), 2.01-1.87(\mathrm{~m}, 1 \mathrm{H})$. 
(+)-5-phenethyldihydrofuran-2(3H)-one $(3 \mathbf{j})^{[24]}$

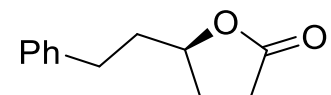

Colorless oil, $97 \%$ yield, 93\% ee, $[\alpha]_{\mathrm{D}}^{25}+55.6$ (c 1.0, chloroform), GC(Supelco $\beta$-DEX ${ }^{\mathrm{TM}}$ $225, \mathrm{df}=0.25 \mu \mathrm{m}, 0.25 \mathrm{~mm}$ i.d. $\times 25 \mathrm{~m}$ ) using a flame ionization detector (FID) operating at $250{ }^{\circ} \mathrm{C}$. Injector temperature was set at $230^{\circ} \mathrm{C}$. The carrier gas was nitrogen with a flow rate of $1.0 \mathrm{~mL} / \mathrm{min}$. The following temperature program was used in the analysis: $190{ }^{\circ} \mathrm{C}$ $(1 \mathrm{~h}), t_{\mathrm{R}}=40.91 \mathrm{~min}$ for major isomer and $t_{\mathrm{R}}=42.61 \mathrm{~min}$ for minor isomer.

$\left.\underline{{ }^{1} \mathrm{H}} \underline{\mathrm{NMR}} \quad \underline{(400} \underline{\mathrm{MHz}}, \underline{\mathrm{CDCl}_{3}}\right)$

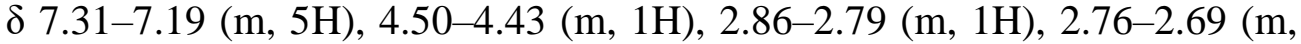
$1 \mathrm{H}), 2.55-2.51(\mathrm{~m}, 2 \mathrm{H}), 2.34-2.26(\mathrm{~m}, 1 \mathrm{H}), 2.09-2.00(\mathrm{~m}, 1 \mathrm{H}), 1.95-1.81(\mathrm{~m}$, $2 \mathrm{H})$.

(+)-5-(2-(benzyloxy)ethyl)dihydrofuran-2(3H)-one (3k)

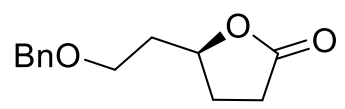

Colorless oil, 99\% yield, $87 \%$ ee, $[\alpha]_{\mathrm{D}}^{25}+37.4$ (c 1.0, chloroform), HPLC condition for corresponding amide: Chiralcel OD-H column $(25 \mathrm{~cm} \times 0.46 \mathrm{~cm}$ ID), $n$-hexane/2-propanol $=80: 20,1.0 \mathrm{~mL} / \mathrm{min}, 214 \mathrm{~nm}$ UV detector, $t_{\mathrm{R}}=10.49 \mathrm{~min}$ (minor) and $t_{\mathrm{R}}=11.89 \mathrm{~min}$ (major).

$\underline{{ }^{1} \mathrm{H}} \underline{\mathrm{NMR}} \quad \underline{(400} \underline{\mathrm{MHz}}, \underline{\left.\mathrm{CDCl}_{3}\right)}$

$\delta 7.49-7.14(\mathrm{~m}, 5 \mathrm{H}), 4.69$ (tdd $, J=8.0,6.5,5.1 \mathrm{~Hz}, 1 \mathrm{H}), 4.57-4.40(\mathrm{~m}, 2 \mathrm{H})$,

$3.70-3.52(\mathrm{~m}, 2 \mathrm{H}), 2.51(\mathrm{dd}, J=9.6,6.8 \mathrm{~Hz}, 2 \mathrm{H}), 2.32(\mathrm{dq}, J=13.4,6.7 \mathrm{~Hz}$, $1 \mathrm{H}), 2.07-1.79(\mathrm{~m}, 3 \mathrm{H})$.

${ }^{13} \mathrm{C} \underline{\mathrm{NMR}} \quad\left(101 \underline{\mathrm{MHz}}, \underline{\mathrm{CDCl}_{3}}\right)$

$\delta 177.04,137.94,128.28,127.57,127.52,78.10,73.07,66.13,35.64,28.66$, 27.96.

$\underline{\mathrm{HRMS}} \underline{(\mathrm{ESI})}$

Calcd for $\left[\mathrm{C}_{13} \mathrm{H}_{16} \mathrm{O}_{3}, \mathrm{M}+\mathrm{NH}_{4}\right]^{+}:$238.1438, Found: 238.1441 .

(+)-5-(4-(benzyloxy)butyl)dihydrofuran-2(3H)-one (31)

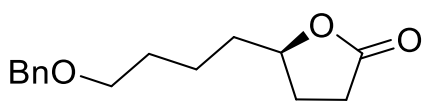

Colorless oil, 99\% yield, 94\% ee, $[\alpha]_{\mathrm{D}}{ }^{25}+25.8$ (c 1.0, chloroform), HPLC condition for corresponding amide: Chiralcel OD-H column $(25 \mathrm{~cm} \times 0.46 \mathrm{~cm}$ ID), $n$-hexane/2-propanol $=90: 10,1.0 \mathrm{~mL} / \mathrm{min}, 214 \mathrm{~nm} \mathrm{UV}$ detector, $t_{\mathrm{R}}=20.11 \mathrm{~min}$ (minor) and $t_{\mathrm{R}}=21.82 \mathrm{~min}$ (major).

$\underline{{ }^{1} \mathrm{H}} \underline{\mathrm{NMR}} \quad \underline{(400} \underline{\mathrm{MHz}}, \underline{\left.\mathrm{CDCl}_{3}\right)}$

$\delta$ 7.41-7.22 (m, 5H), 4.57-4.40 (m, 3H), $3.48(\mathrm{t}, J=6.3 \mathrm{~Hz}, 2 \mathrm{H}), 2.51(\mathrm{dd}, J$ $=9.5,6.9 \mathrm{~Hz}, 2 \mathrm{H}), 2.29(\mathrm{dq}, J=13.4,6.7 \mathrm{~Hz}, 1 \mathrm{H}), 1.93-1.34(\mathrm{~m}, 7 \mathrm{H})$.

${ }^{13} \mathrm{C} \underline{\mathrm{NMR}} \quad\left(101 \mathrm{MHz}_{2} \underline{\mathrm{CDCl}_{3}}\right)$

$\delta 177.14,138.35,128.25,127.51,127.43,80.78,72.81,69.81,35.21,29.30$, 28.70, 27.83, 21.96.

$\underline{\operatorname{HRMS}} \underline{(\mathrm{ESI})}$

Calcd for $\left[\mathrm{C}_{15} \mathrm{H}_{20} \mathrm{O}_{3}, \mathrm{M}+\mathrm{H}\right]^{+}: 249.1485$, Found: 249.1491 . 
(+)-5-(4-methylpent-3-enyl)dihydrofuran-2(3H)-one (3m)

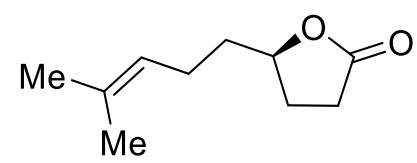

Colorless oil, $91 \%$ yield, $93 \%$ ee, $[\alpha]_{\mathrm{D}}^{25}+46.0$ (c 1.0, chloroform), GC(Supelco $\beta$-DEX ${ }^{\mathrm{TM}}$ $225, \mathrm{df}=0.25 \mu \mathrm{m}, 0.25 \mathrm{~mm}$ i.d. $\times 25 \mathrm{~m}$ ) using a flame ionization detector (FID) operating at $250{ }^{\circ} \mathrm{C}$. Injector temperature was set at $230{ }^{\circ} \mathrm{C}$. The carrier gas was nitrogen with a flow rate of $1.0 \mathrm{~mL} / \mathrm{min}$. The following temperature program was used in the analysis: $100{ }^{\circ} \mathrm{C}$ $5{ }^{\circ} \mathrm{C} / \mathrm{min}-180{ }^{\circ} \mathrm{C}-2{ }^{\circ} \mathrm{C} / \mathrm{min}-200{ }^{\circ} \mathrm{C}(4 \mathrm{~min}), t_{\mathrm{R}}=17.95 \mathrm{~min}$ for major isomer and $t_{\mathrm{R}}=$ $18.13 \mathrm{~min}$ for minor isomer.

$\left.\underline{{ }^{1} \mathrm{H}} \underline{\mathrm{NMR}} \quad \underline{(400} \underline{\mathrm{MHz}}, \underline{\mathrm{CDCl}_{3}}\right)$

$\delta 5.10(\mathrm{t}, J=7.0 \mathrm{~Hz}, 1 \mathrm{H}), 4.49(\mathrm{dt}, J=14.5,7.6 \mathrm{~Hz}, 1 \mathrm{H}), 2.53(\mathrm{dd}, J=9.5,6.9$

$\mathrm{Hz}, 2 \mathrm{H}), 2.32(\mathrm{dq}, J=13.5,6.8 \mathrm{~Hz}, 1 \mathrm{H}), 2.13(\mathrm{q}, J=7.6 \mathrm{~Hz}, 2 \mathrm{H}), 1.94-1.74$

$(\mathrm{m}, 2 \mathrm{H}), 1.70(\mathrm{~s}, 3 \mathrm{H}), 1.67-1.54(\mathrm{~m}, 4 \mathrm{H})$.

${ }^{13} \mathrm{C} \underline{\mathrm{NMR}} \quad \underline{(101} \underline{\mathrm{MHz}}, \underline{\left.\mathrm{CDCl}_{3}\right)}$

$\delta 177.21,132.85,122.68,80.41,35.60,28.81,27.92,25.62,23.81,17.61$.

$\underline{\text { HRMS }} \underline{(\mathrm{ESI})}$

Calcd for $\left[\mathrm{C}_{10} \mathrm{H}_{16} \mathrm{O}_{2}, \mathrm{M}+\mathrm{H}\right]^{+}:$169.1223, Found: 169.1219 .

(+)-ethyl 3-(5-oxotetrahydrofuran-2-yl)propanoate $(30)^{[25]}$

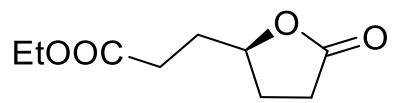

Colorless oil, $91 \%$ yield, $93 \%$ ee, $[\alpha]_{\mathrm{D}}{ }^{25}+47.9$ (c 1.0, chloroform), GC(Supelco $\beta$-DEX ${ }^{\mathrm{TM}}$ $225, \mathrm{df}=0.25 \mu \mathrm{m}, 0.25 \mathrm{~mm}$ i.d. $\times 25 \mathrm{~m}$ ) using a flame ionization detector (FID) operating at $250{ }^{\circ} \mathrm{C}$. Injector temperature was $230{ }^{\circ} \mathrm{C}$. The carrier gas was nitrogen with a flow rate of $1.0 \mathrm{~mL} / \mathrm{min}$. The following temperature program was used in the analysis: $150{ }^{\circ} \mathrm{C}(1 \mathrm{~h})$, $t_{\mathrm{R}}=27.32 \mathrm{~min}$ for major isomer and $t_{\mathrm{R}}=27.87 \mathrm{~min}$ for minor isomer.

$\left.{ }^{1} \mathrm{H} \underline{\mathrm{NMR}} \quad \underline{(400} \underline{\mathrm{MHz}}, \underline{\mathrm{CDCl}_{3}}\right)$

$\delta 4.59-4.47(\mathrm{~m}, 1 \mathrm{H}), 4.11(\mathrm{q}, J=7.1 \mathrm{~Hz}, 2 \mathrm{H}), 2.58-2.43(\mathrm{~m}, 4 \mathrm{H}), 2.41-2.27$

$(\mathrm{m}, 1 \mathrm{H}), 2.02-1.79(\mathrm{~m}, 3 \mathrm{H}), 1.23(\mathrm{t}, J=7.0 \mathrm{~Hz}, 3 \mathrm{H})$.

\section{(S)-5-phenyldihydrofuran-2(3H)-one ${ }^{[26]}$}

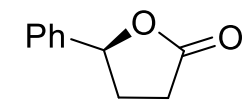

Colorless oil, $94 \%$ yield, $80 \%$ ee, $[\alpha]_{\mathrm{D}}{ }^{25}+14.2$ (c 1.0, dichloromethane), HPLC condition for corresponding amide: Chiralcel OB column $(25 \mathrm{~cm} \times 0.46 \mathrm{~cm}$ ID), $n$-hexane/2-propanol $=80: 20,1.0 \mathrm{~mL} / \mathrm{min}, 214 \mathrm{~nm} \mathrm{UV}$ detector, $t_{\mathrm{R}}=14.45 \mathrm{~min}(S)$ and $t_{\mathrm{R}}=19.59 \mathrm{~min}(R)$. $\underline{{ }^{1} \mathrm{H}} \underline{\mathrm{NMR}} \underline{(400} \underline{\mathrm{MHz}}, \underline{\left.\mathrm{CDCl}_{3}\right)}$

$\delta$ 7.44-7.29 (m, 5H), $5.51(\mathrm{dd}, J=7.6$ and $6.4 \mathrm{~Hz}, 1 \mathrm{H}), 2.73-2.57(\mathrm{~m}, 3 \mathrm{H})$, $2.28-2.11(\mathrm{~m}, 1 \mathrm{H})$.

\section{4-hydroxy-N-phenylpentanamide ${ }^{[27]}$}<smiles>CC(O)CCC(=O)Nc1ccccc1</smiles> 
Colorless oil, 53\% yield, 14\% ee, HPLC condition for corresponding amide: Chiralcel OD3 column $(25 \mathrm{~cm} \times 0.46 \mathrm{~cm} \mathrm{ID}), n$-hexane $/ 2$-propanol $=95: 5,0.5 \mathrm{~mL} / \mathrm{min}, 210 \mathrm{~nm} \mathrm{UV}$ detector, $t_{\mathrm{R}}=34.12 \mathrm{~min}$ and $t_{\mathrm{R}}=36.09 \mathrm{~min}$.

$\underline{{ }^{1} \mathrm{H}} \underline{\mathrm{NMR}}\left(400 \underline{\mathrm{MHz}}, \underline{\left.\mathrm{CDCl}_{3}\right)}\right.$

$\delta 8.03(\mathrm{~s}, 1 \mathrm{H}), 7.50(\mathrm{~d}, J=7.9 \mathrm{~Hz}, 2 \mathrm{H}), 7.36-7.23(\mathrm{~m}, 2 \mathrm{H}), 7.09(\mathrm{t}, J=7.4$ $\mathrm{Hz}, 1 \mathrm{H}), 3.97-3.79(\mathrm{~m}, 1 \mathrm{H}), 3.01(\mathrm{~s}, 1 \mathrm{H}), 2.52$ (t, $J=7.1 \mathrm{~Hz}, 2 \mathrm{H}), 2.02-$ $1.84(\mathrm{~m}, 1 \mathrm{H}), 1.84-1.68(\mathrm{~m}, 1 \mathrm{H}), 1.22(\mathrm{~d}, J=6.3 \mathrm{~Hz}, 3 \mathrm{H})$.

\section{1-(3-hydroxybutyl)-3-phenylurea}

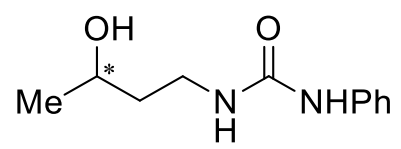

Colorless oil, $42 \%$ yield, $27 \%$ ee, HPLC condition for corresponding amide: Chiralcel OD$\mathrm{H}$ column $(25 \mathrm{~cm} \times 0.46 \mathrm{~cm} \mathrm{ID}), n$-hexane $/ 2$-propanol $=80: 20,1.0 \mathrm{~mL} / \mathrm{min}, 254 \mathrm{~nm} \mathrm{UV}$ detector, $t_{\mathrm{R}}=5.99 \mathrm{~min}$ and $t_{\mathrm{R}}=9.11 \mathrm{~min}$.

${ }^{1} \mathrm{H} \underline{\mathrm{NMR}}\left(400 \mathrm{MHz}, \underline{\left.\mathrm{DMSO}-d_{6}\right)}\right.$

$\delta 8.46(\mathrm{~s}, 1 \mathrm{H}), 7.38(\mathrm{~d}, J=7.2 \mathrm{~Hz}, 2 \mathrm{H}), 7.28-7.14(\mathrm{~m}, 2 \mathrm{H}), 6.88(\mathrm{t}, J=7.3$

$\mathrm{Hz}, 1 \mathrm{H}), 6.12(\mathrm{t}, J=5.7 \mathrm{~Hz}, 1 \mathrm{H}), 4.55(\mathrm{~d}, J=4.6 \mathrm{~Hz}, 1 \mathrm{H}), 3.76-3.59(\mathrm{~m}, 1 \mathrm{H})$,

$3.15(\mathrm{q}, J=6.6 \mathrm{~Hz}, 2 \mathrm{H}), 1.60-1.38(\mathrm{~m}, 2 \mathrm{H}), 1.08(\mathrm{~d}, J=6.2 \mathrm{~Hz}, 3 \mathrm{H})$.

${ }^{13} \mathrm{C}$ NMR $\quad\left(101 \mathrm{MHz}\right.$, DMSO- $\left.d_{6}\right)$

$\delta 155.8,141.1,129.1,121.3,118.0,64.5,36.9,24.2$.

$\underline{\text { HRMS }} \underline{(E S I)}$

Calcd for $\left[\mathrm{C}_{11} \mathrm{H}_{16} \mathrm{~N}_{2} \mathrm{O}_{2}, \mathrm{M}+\mathrm{H}\right]^{+}:$209.1285, Found: 209.1280. 


\section{Mechanism Experiments and NMR Studies.}

\section{intermediate $6^{[28]}$}

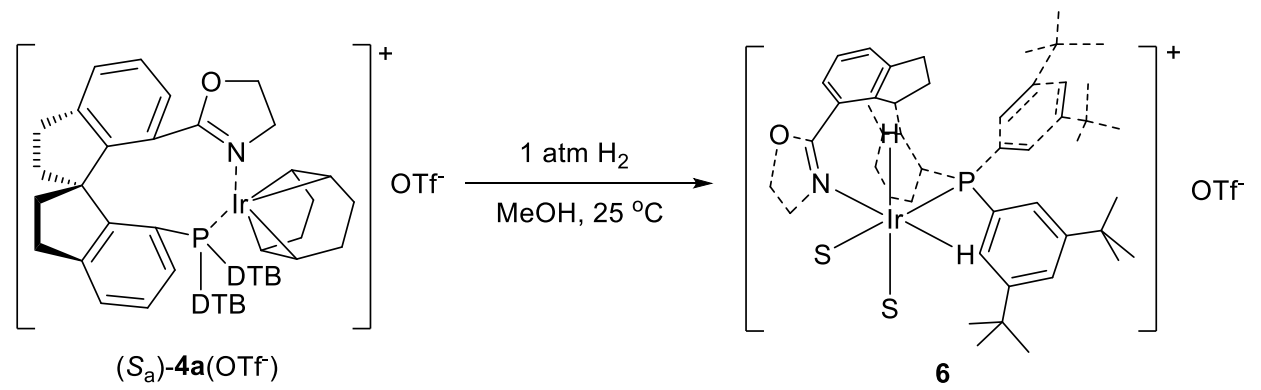

In a glove-box, iridium complex $\left(S_{\mathrm{a}}\right)-\mathbf{4 a}\left(\mathrm{OTf}^{-}\right)(300 \mathrm{mg}, 0.26 \mathrm{mmol})$ and $\mathrm{MeOH}(3$ $\mathrm{mL})$ were added into a Schlenk tube. The mixture was frozen with liquid nitrogen and was replaced with hydrogen gas for five times. The Schlenk tube was then charged with hydrogen to $1 \mathrm{~atm}$ by a balloon and the reaction mixture was stirred at $25{ }^{\circ} \mathrm{C}$ for $0.5 \mathrm{~h}$. When the system turned from orange to pale yellow, the solvent of reaction was removed in a vacuum, and $\mathrm{MeOH}-d_{4}$ was added. The resulted solution was transferred into an NMR tube in a glove-box and measured the NMR spectra of the dihydride intermediate $6(>95 \%$ yield by NMR). The NMR spectra of $\mathbf{6}$ are consistent with those obtained by our previous methods. ${ }^{[28]}$

\section{intermediate 7}
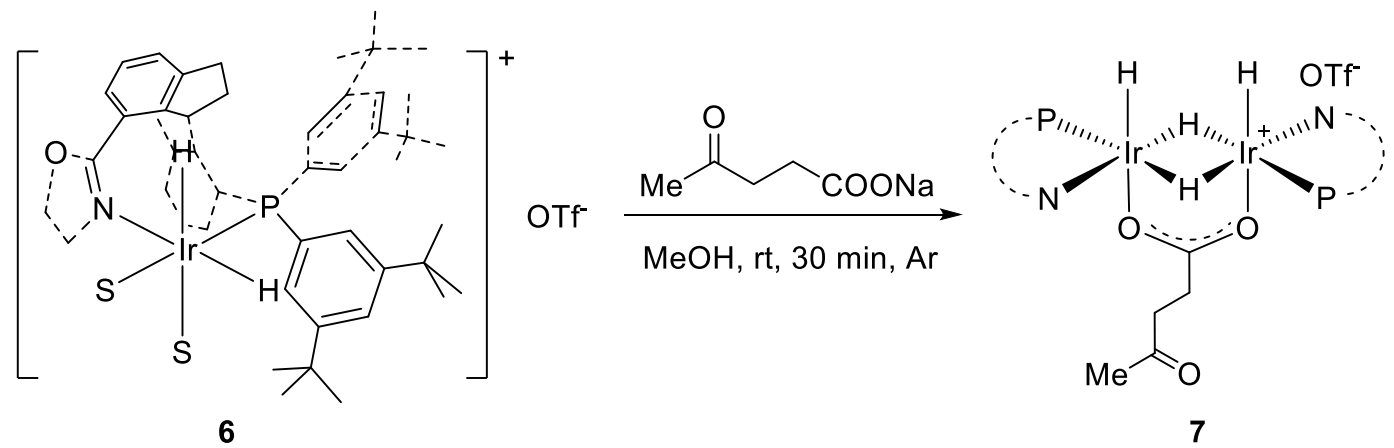

A solution of sodium 4-oxopentanoate $(36 \mathrm{mg}, 0.26 \mathrm{mmol})$ in $\mathrm{MeOH}(1 \mathrm{~mL})$ was added to the solution of 6 in $\mathrm{MeOH}(0.09 \mathrm{mmol} / \mathrm{L}, 3 \mathrm{~mL})$ under argon atmosphere. The mixture was stirred at $25^{\circ} \mathrm{C}$ for $0.5 \mathrm{~h}$ until the system turned from pale yellow to orange, and was filtrated through celite. The filtrate was concentrated in vacuum to give product 7 (>95\% by NMR).

${ }^{1} \mathrm{H} \underline{\mathrm{NMR}} \quad \underline{(400} \underline{\mathrm{MHz}}, \underline{\left.\mathrm{MeOH}-d_{4}\right)}$

$\delta 8.23(\mathrm{dd}, J=13.5,7.9 \mathrm{~Hz}, 2 \mathrm{H}), 7.83(\mathrm{~d}, J=15.7 \mathrm{~Hz}, 2 \mathrm{H}), 7.61(\mathrm{~d}, J=7.2$ $\mathrm{Hz}, 2 \mathrm{H}), 7.52(\mathrm{t}, J=7.7 \mathrm{~Hz}, 2 \mathrm{H}), 7.42(\mathrm{~d}, J=1.8 \mathrm{~Hz}, 2 \mathrm{H}), 7.36(\mathrm{t}, J=7.5 \mathrm{~Hz}$, 2H), $7.30(\mathrm{~d}, J=11.6 \mathrm{~Hz}, 6 \mathrm{H}), 7.10(\mathrm{~d}, J=7.6 \mathrm{~Hz}, 2 \mathrm{H}), 6.93(\mathrm{~d}, J=8.0 \mathrm{~Hz}$, $4 \mathrm{H}), 4.15$ (dt, $J=9.9,7.5 \mathrm{~Hz}, 2 \mathrm{H}), 3.44$ (dd, $J=9.6,1.5 \mathrm{~Hz}, 2 \mathrm{H}), 3.19-3.09$ $(\mathrm{m}, 4 \mathrm{H}), 2.92(\mathrm{dd}, J=15.7,8.3 \mathrm{~Hz}, 2 \mathrm{H}), 2.74-2.59(\mathrm{~m}, 6 \mathrm{H}), 2.46-2.36(\mathrm{~m}$, $3 \mathrm{H}), 2.15(\mathrm{~s}, 3 \mathrm{H}), 2.14-1.95(\mathrm{~m}, 9 \mathrm{H}), 1.49(\mathrm{~s}, 18 \mathrm{H}), 1.11(\mathrm{~s}, 36 \mathrm{H}), 1.03(\mathrm{~s}$, $18 \mathrm{H}),-18.68(\mathrm{dd}, J=58.4,12.6 \mathrm{~Hz}, 2 \mathrm{H}),-24.01(\mathrm{~d}, J=19.1 \mathrm{~Hz}, 2 \mathrm{H})$. 
$\underline{{ }^{31} \mathrm{P} N M R} \quad \underline{(161} \underline{\mathrm{MHz}}, \underline{\left.\mathrm{MeOH}-d_{4}\right)}$

$\delta 10.4(\mathrm{~s})$

\section{intermediate 8}

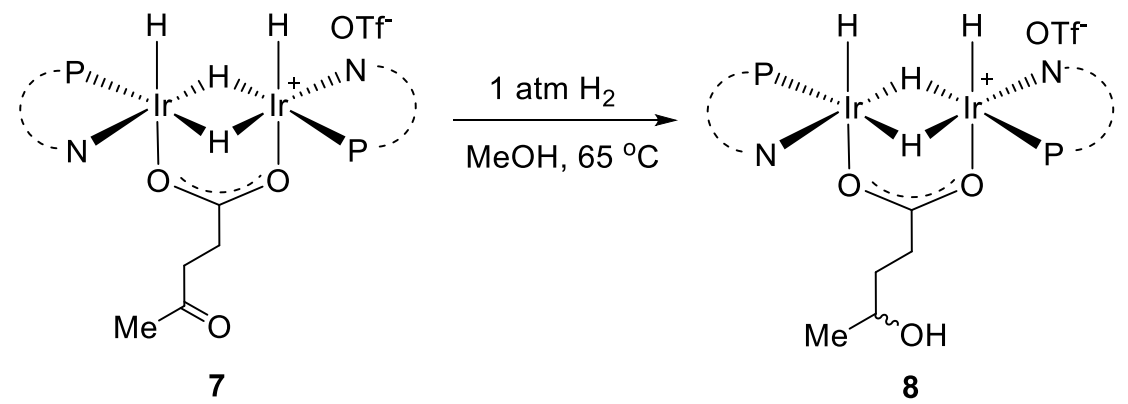

The compound 8 was made by heating intermediates 7 in $\mathrm{MeOH}$ under $\mathrm{H}_{2}$ (1 atm) at $65{ }^{\circ} \mathrm{C}$ for $48 \mathrm{~h}$. When a solution of the intermediate 7 in methanol was treated with $1 \mathrm{~atm}$ $\mathrm{H}_{2}$ at $65^{\circ} \mathrm{C}$ for 48 hours, a set of new iridium hydride signals appeared. By analogy to our previous observations ${ }^{[28,29]}$, we attributed these new signals to the diiridium complex $\mathbf{8}$, an intermediate that the hydrogenation product bridges two iridium centers. We conducted ${ }^{1} \mathrm{H}$ NMR to track the hydrogenation reaction (Figure S4). When a solution of 6 in $\mathrm{MeOH}$ was treated with sodium 4-oxopentanoate at $25^{\circ} \mathrm{C}$, the signal of 7 appeared (Figure S4b). The intermediate 7 was slowly converted to the intermediate 8 under 1 atm hydrogen at $65^{\circ} \mathrm{C}$ (Figure S4c, Figure S4d and Figure S4e).

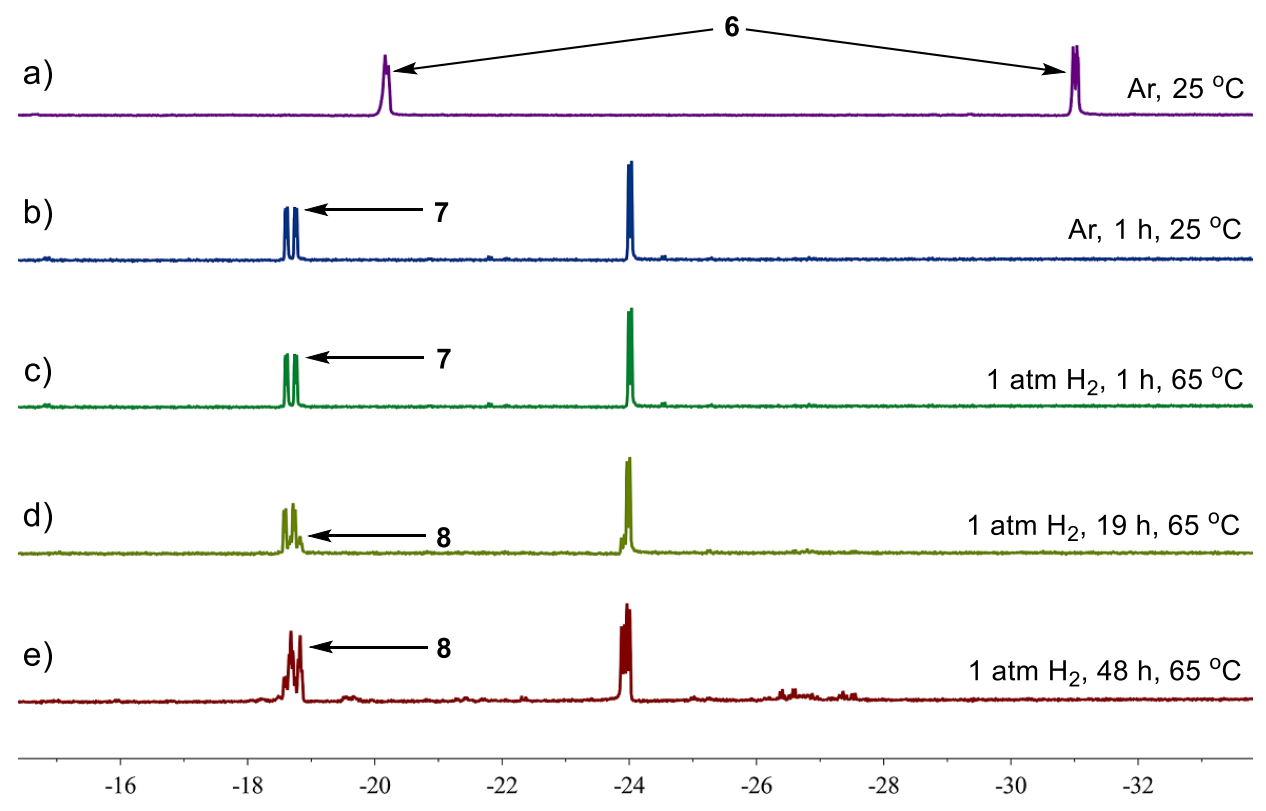

Figure S4. ${ }^{1} \mathrm{H}$ NMR analysis of the hydrogenation in $\mathrm{CD}_{3} \mathrm{OD}$. 


\section{NMR Spectra of New Compounds}

\section{${ }^{1} \mathrm{H}$ NMR of $\left(S_{\mathrm{a}, S}\right)-\mathrm{S} 4$}

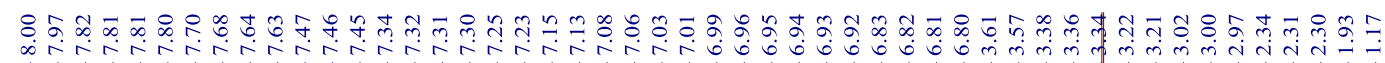

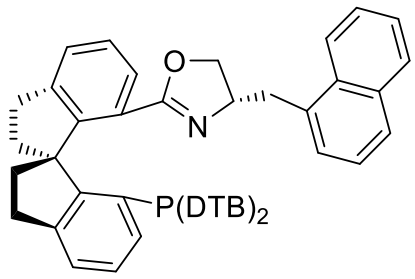

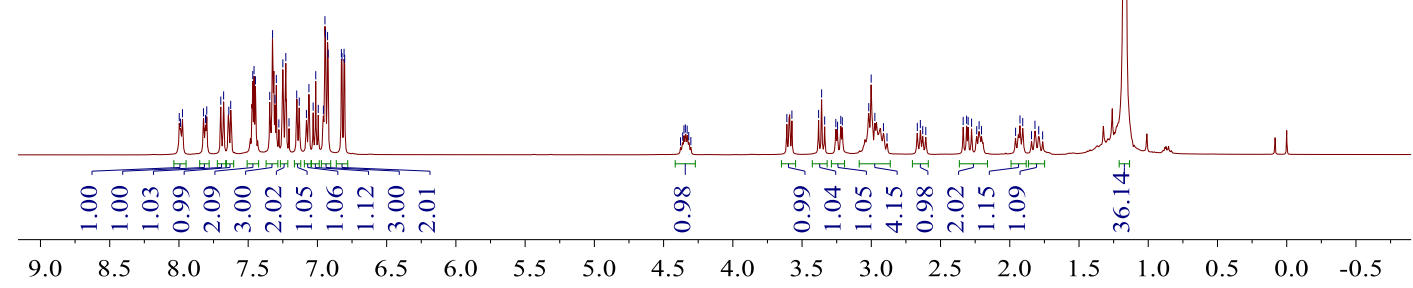

\section{${ }^{13} \mathrm{C}$ NMR of $\left(S_{\mathrm{a}}, S\right)-\mathrm{S} 4$}

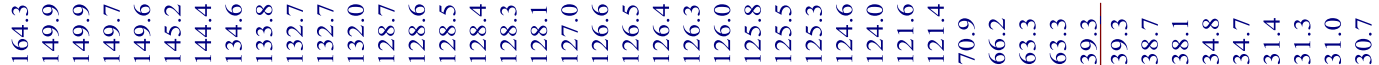

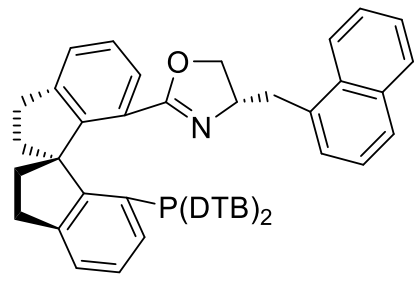

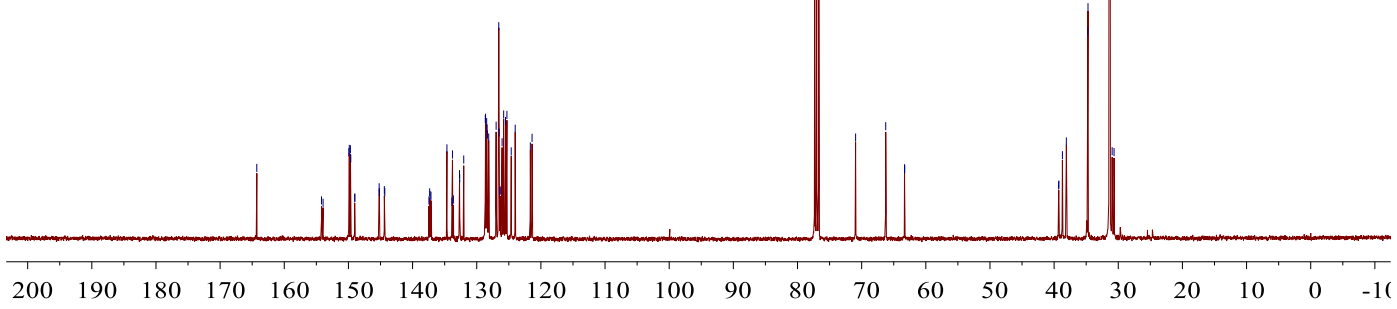




\section{${ }^{31} \mathrm{P}$ NMR of $\left(S_{\mathrm{a}}, S\right)-\mathrm{S} 4$}

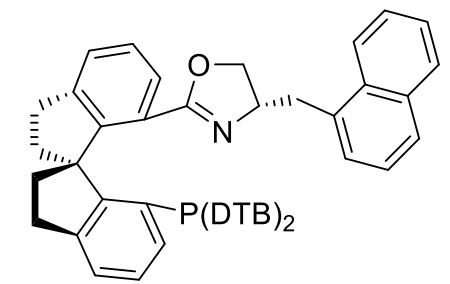

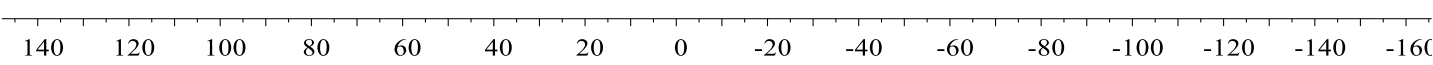

\section{${ }^{1} \mathrm{H}$ NMR of $(\mathrm{Sa}, \mathrm{S})-4 \mathrm{e}$}

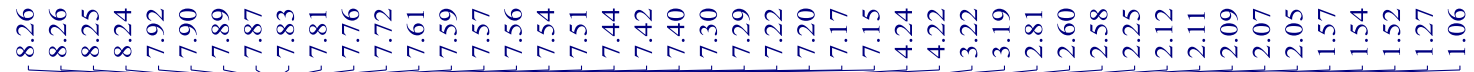

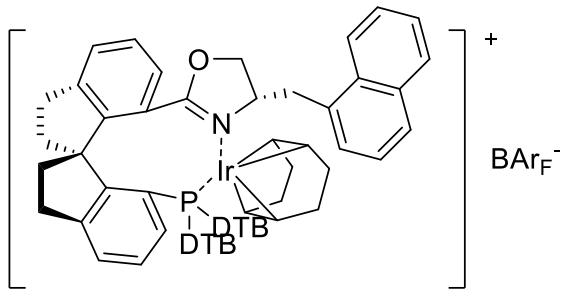

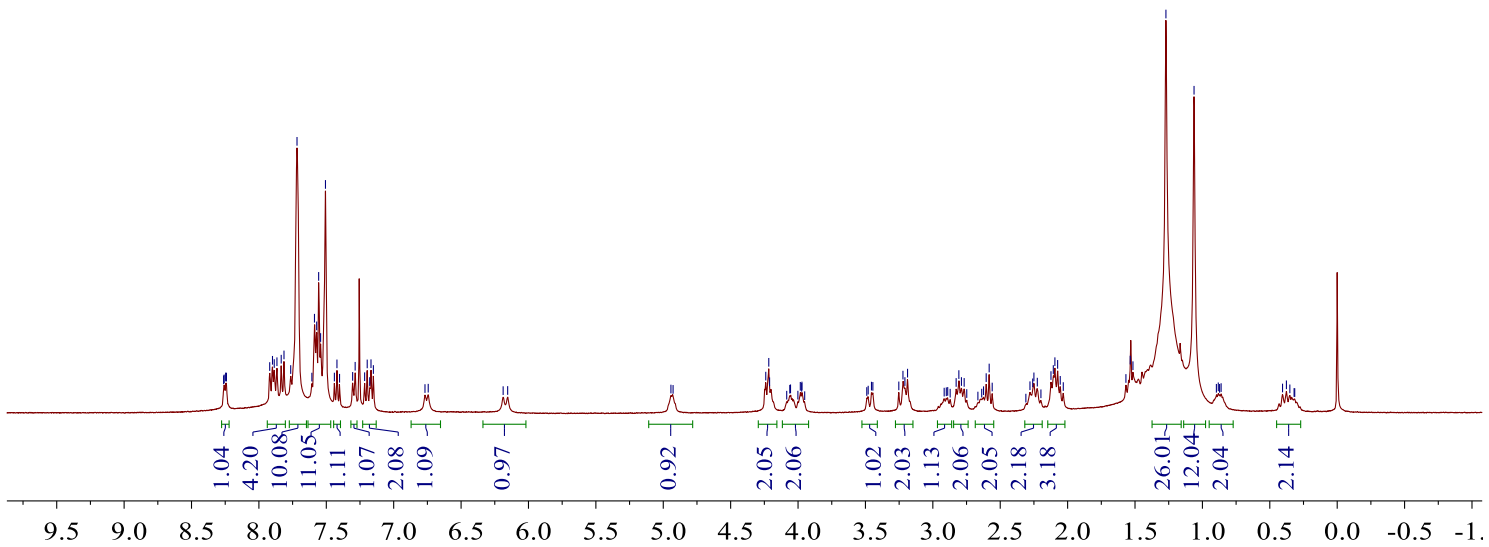




\section{${ }^{13} \mathrm{C}$ NMR of $(\mathrm{Sa}, \mathrm{S})-4 \mathrm{e}$}

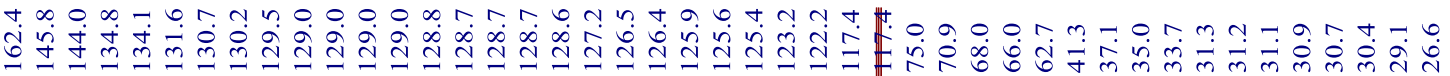

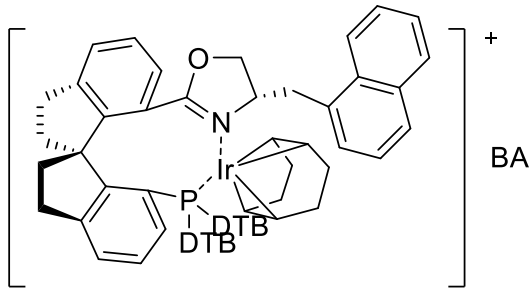

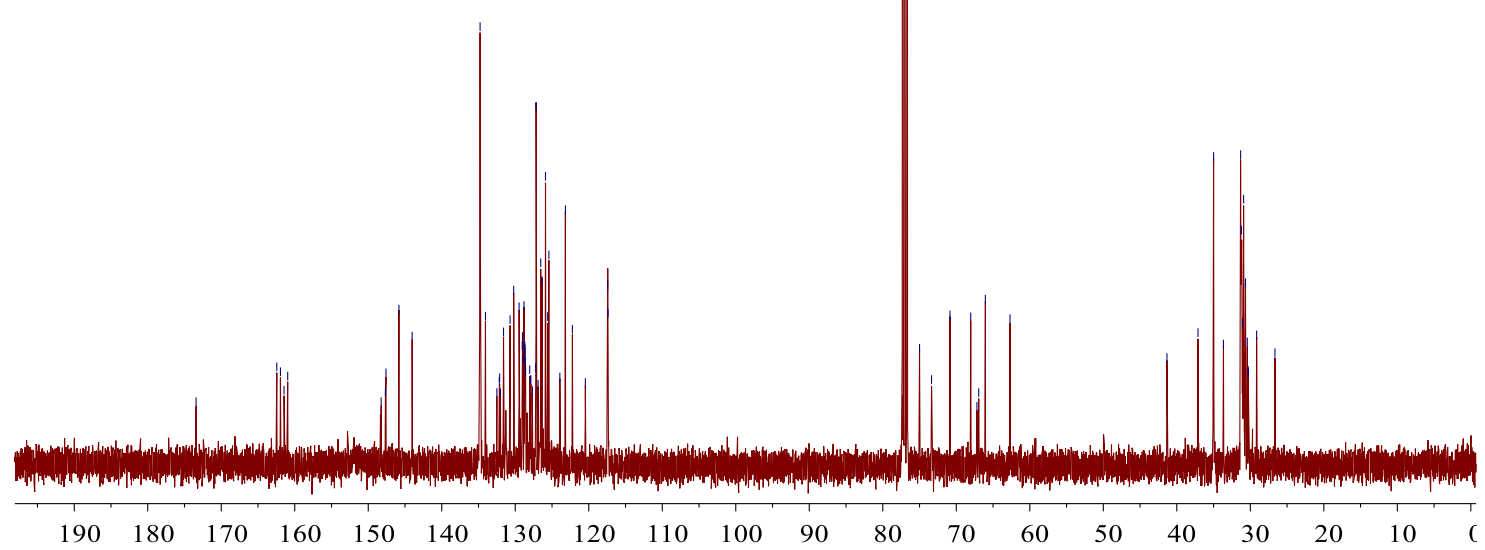

${ }^{31} \mathrm{P}$ NMR of $(S a, S)-4 \mathrm{e}$

$\frac{n}{1}$

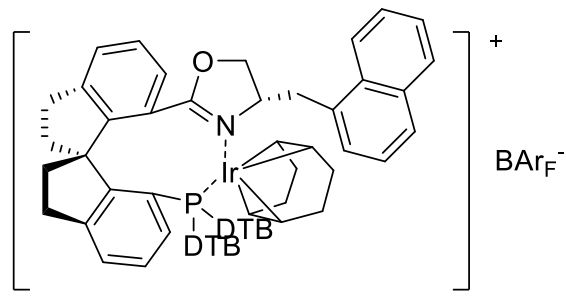

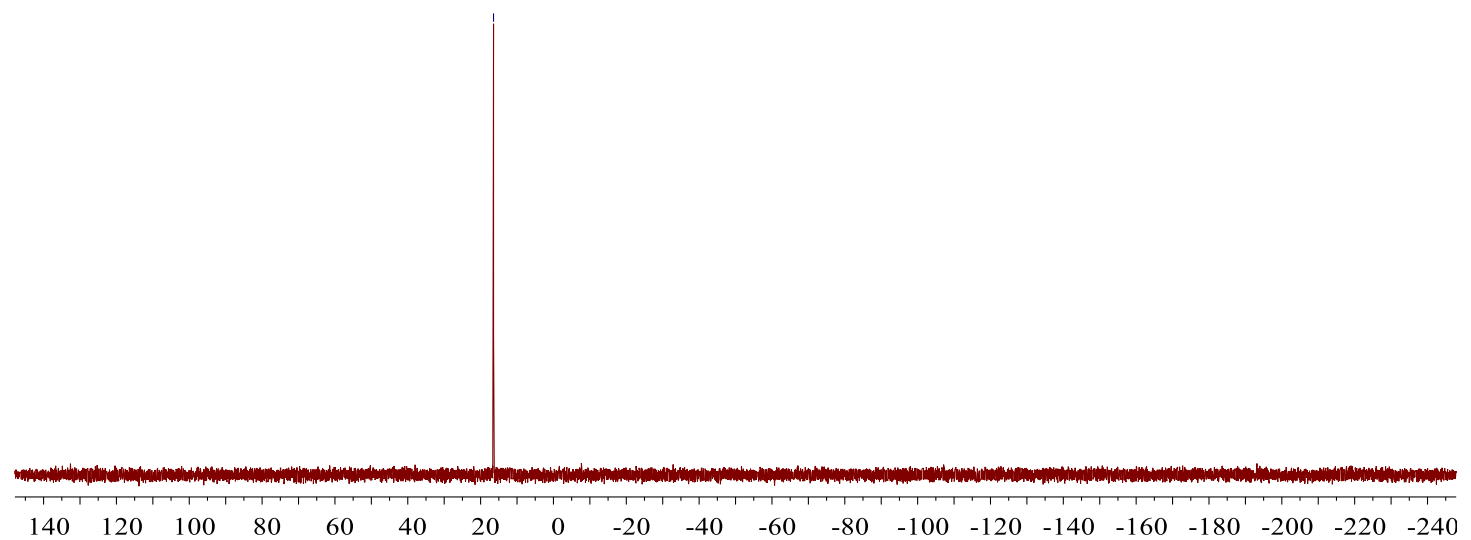




\section{${ }^{1}$ H NMR of 6}

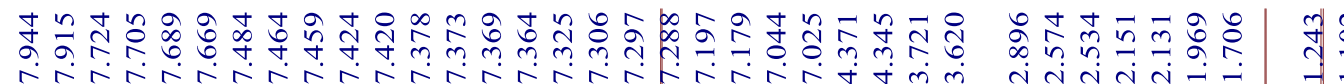

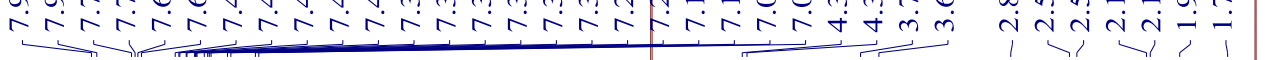

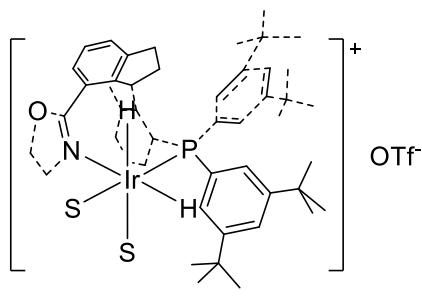

6

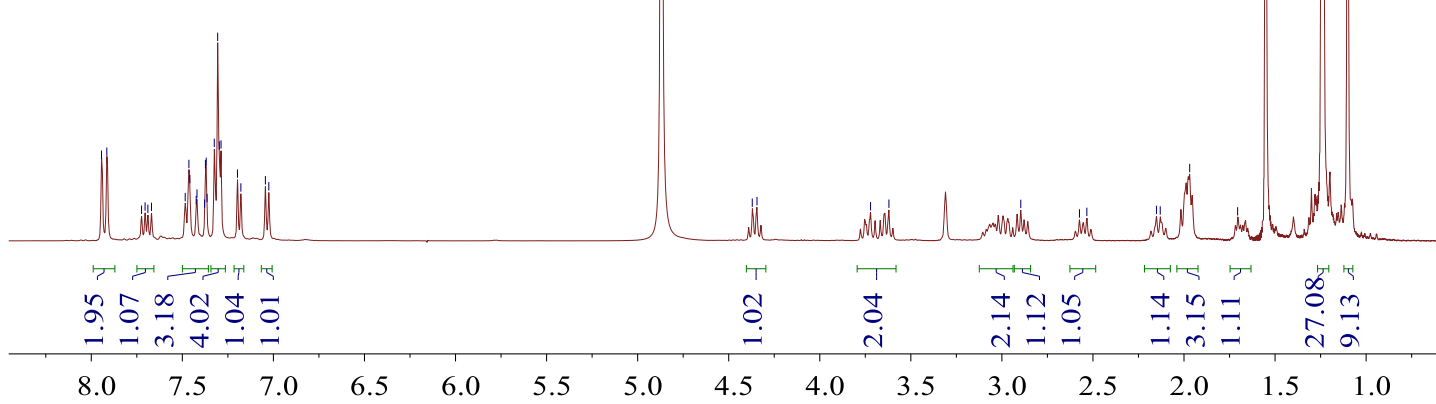

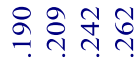

ํํतํํ

そั0ิ

के के ले

$\longrightarrow$

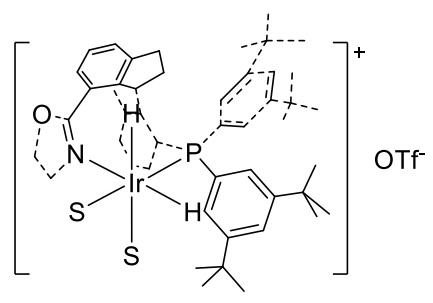

6

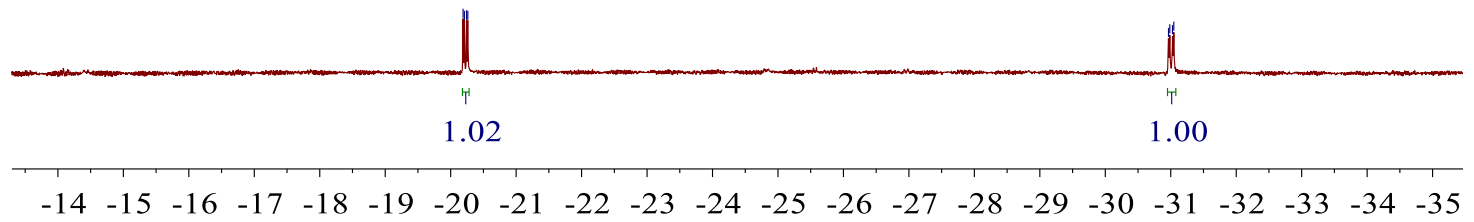


${ }^{31}$ P NMR of 6

N
N
1

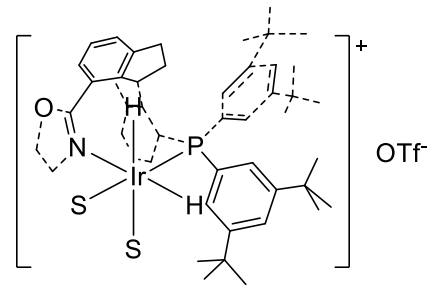

6

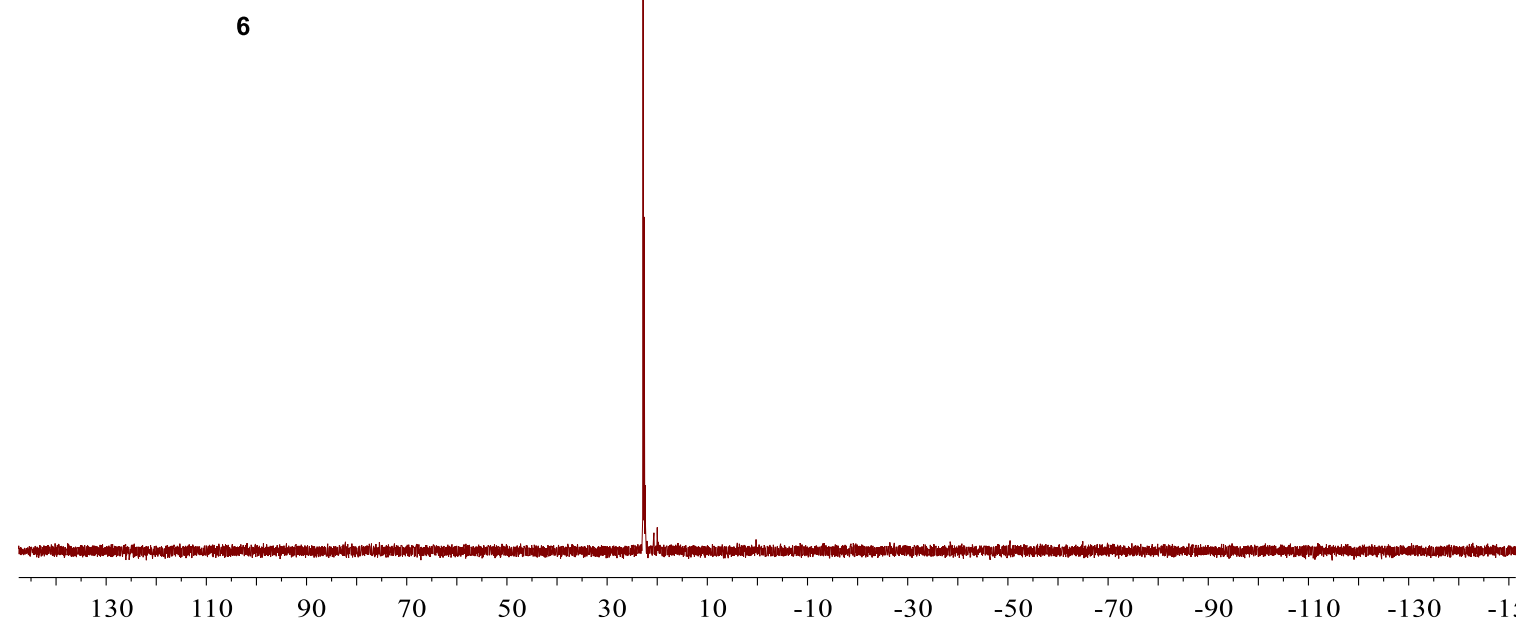




\section{${ }^{1} \mathrm{H}$ NMR of 7}

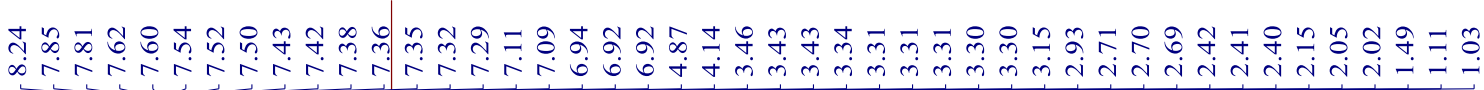
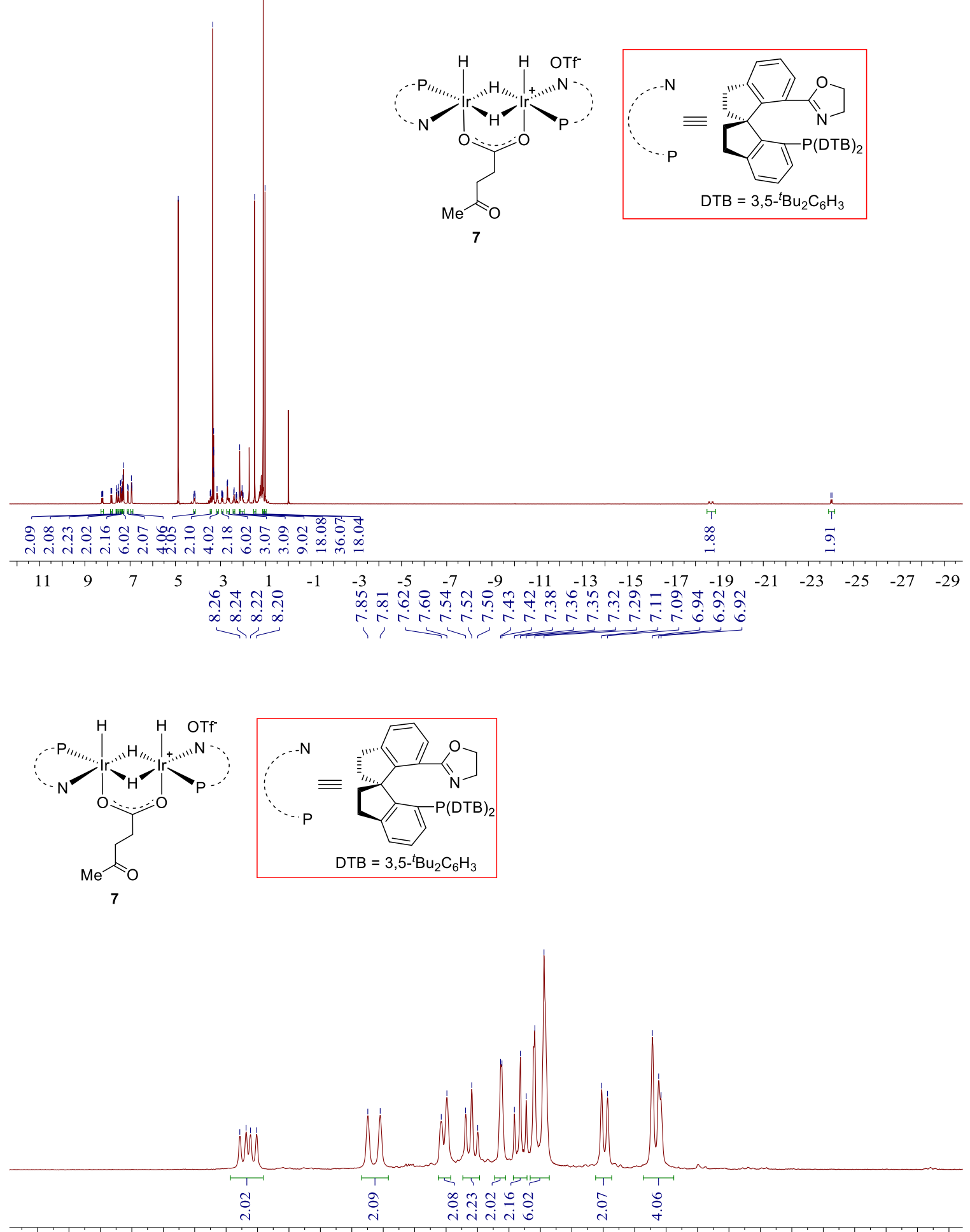

$\begin{array}{llllllllllllllllllllllllllllllll}8.9 & 8.8 & 8.7 & 8.6 & 8.5 & 8.4 & 8.3 & 8.2 & 8.1 & 8.0 & 7.9 & 7.8 & 7.7 & 7.6 & 7.5 & 7.4 & 7.3 & 7.2 & 7.1 & 7.0 & 6.9 & 6.8 & 6.7 & 6.6 & 6.5 & 6.4 & 6.3 & 6.2 & 6.1 & 6.0\end{array}$ 

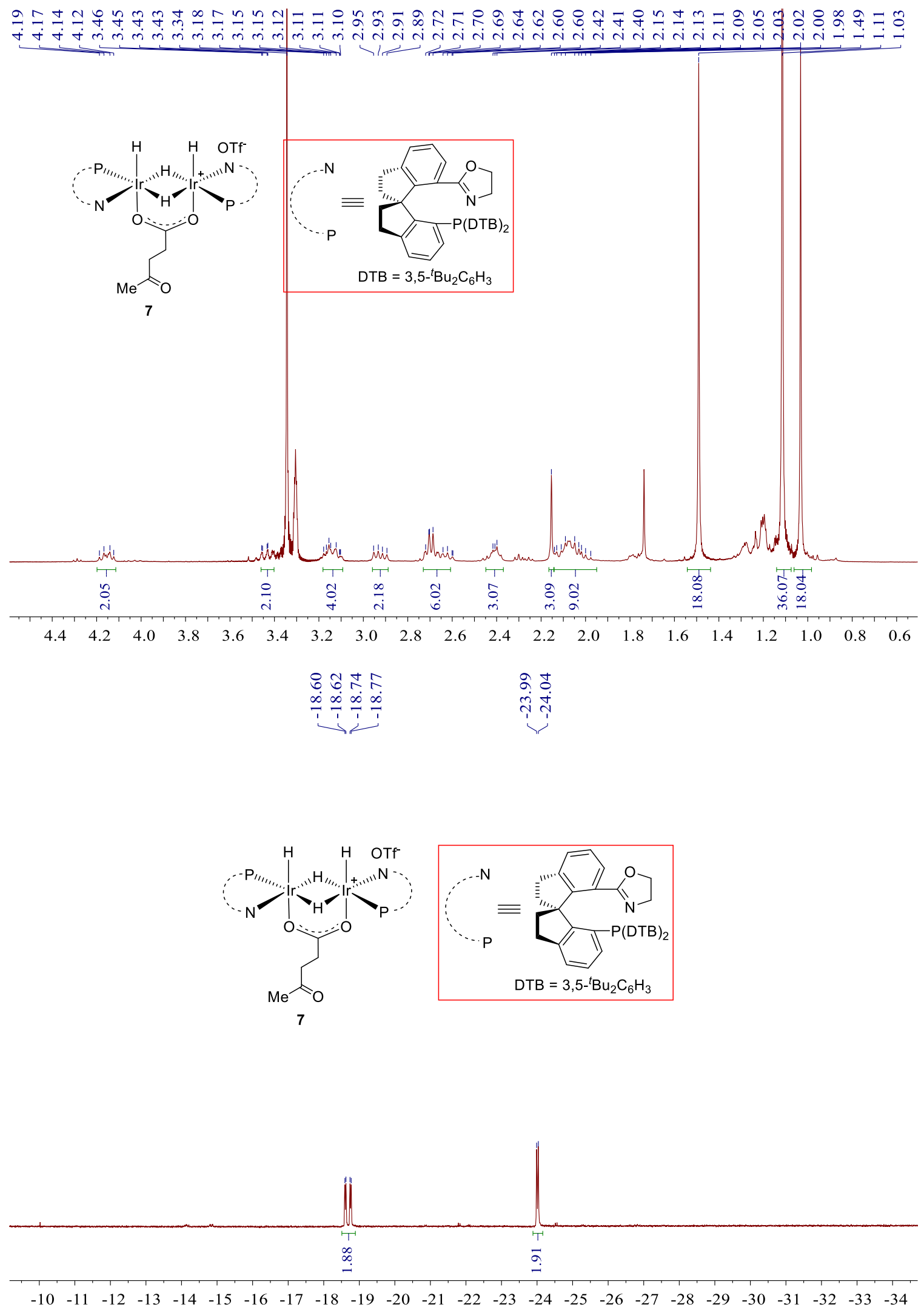


\section{${ }^{31}$ P NMR of 7}

$$
\underset{1}{\stackrel{7}{\circ}}
$$
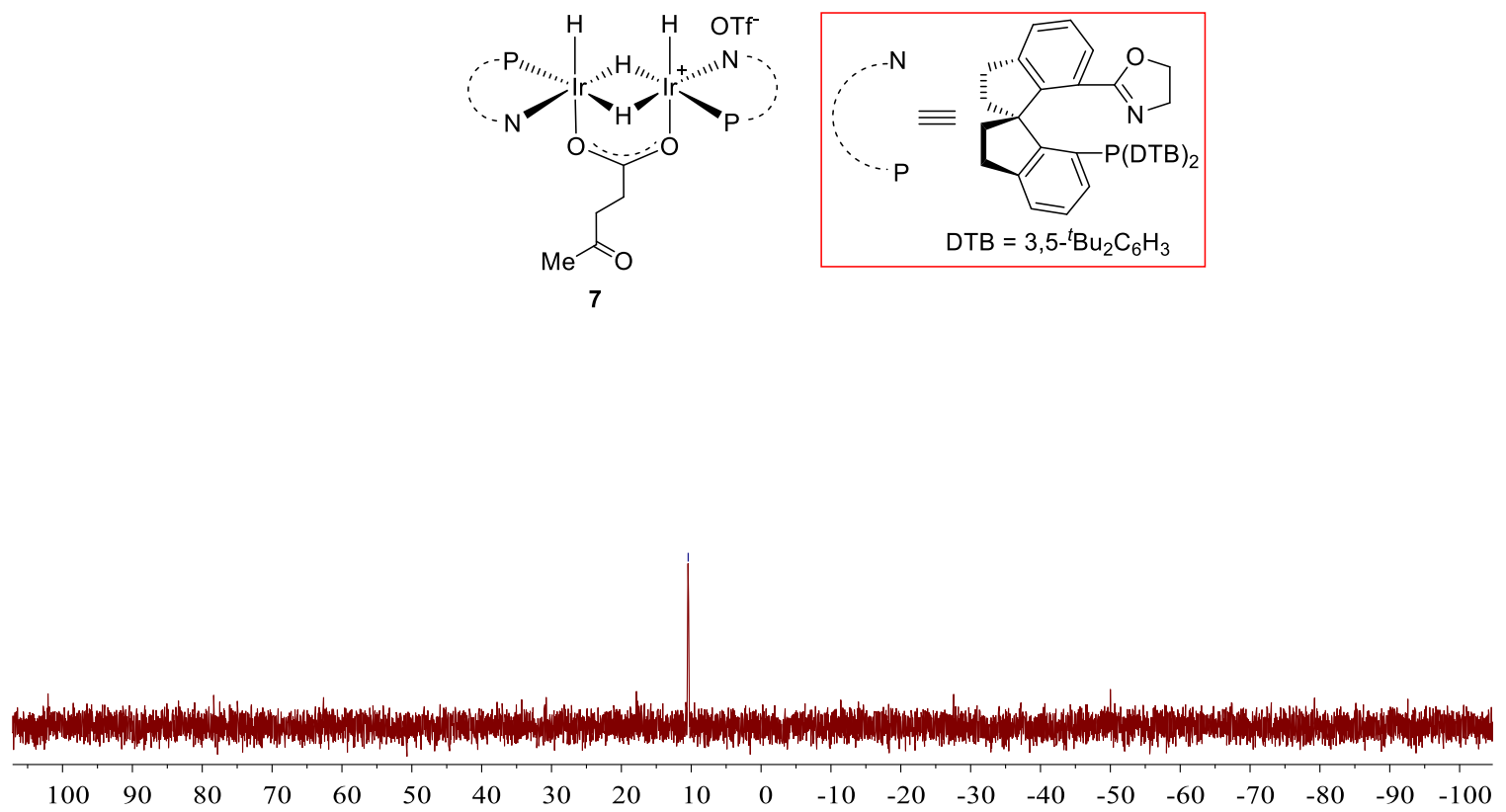


\section{8-(benzyloxy)-4-oxooctanoic acid (11)}

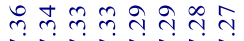

ringing

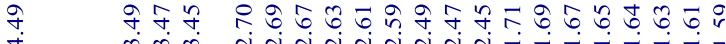

i.<smiles>O=C(O)CCCC(=O)CCCCOCc1ccccc1</smiles>

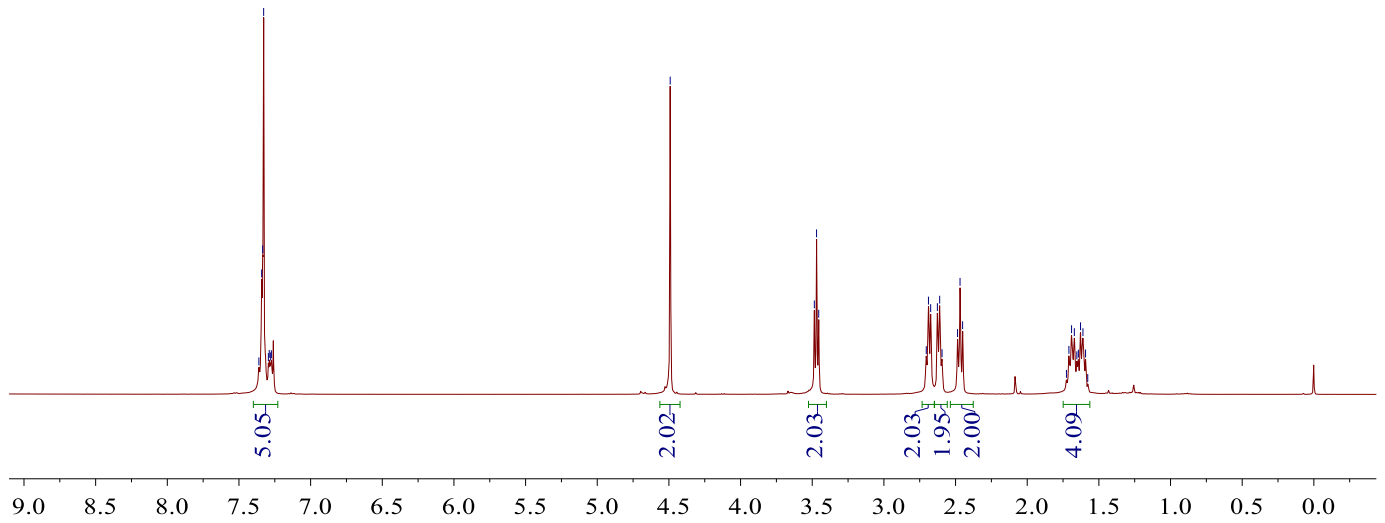

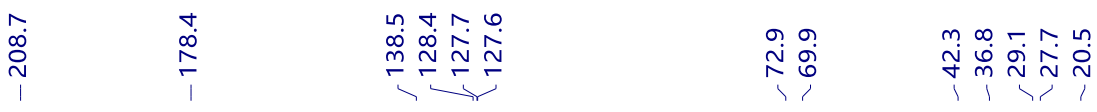<smiles>O=C(O)CCC(=O)CCCCOCc1ccccc1</smiles>

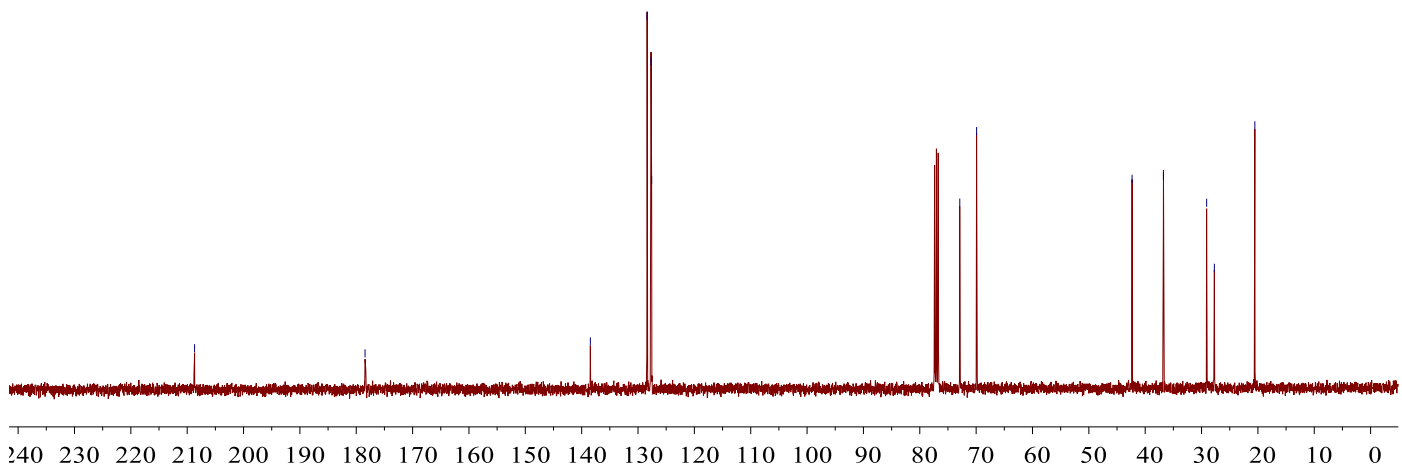


(+)-5-(2-(benzyloxy)ethyl)dihydrofuran-2(3H)-one (3k)

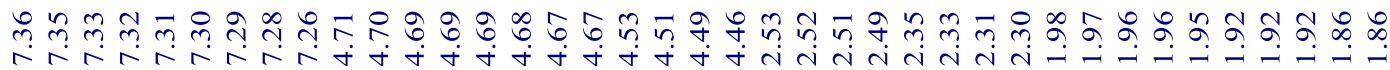

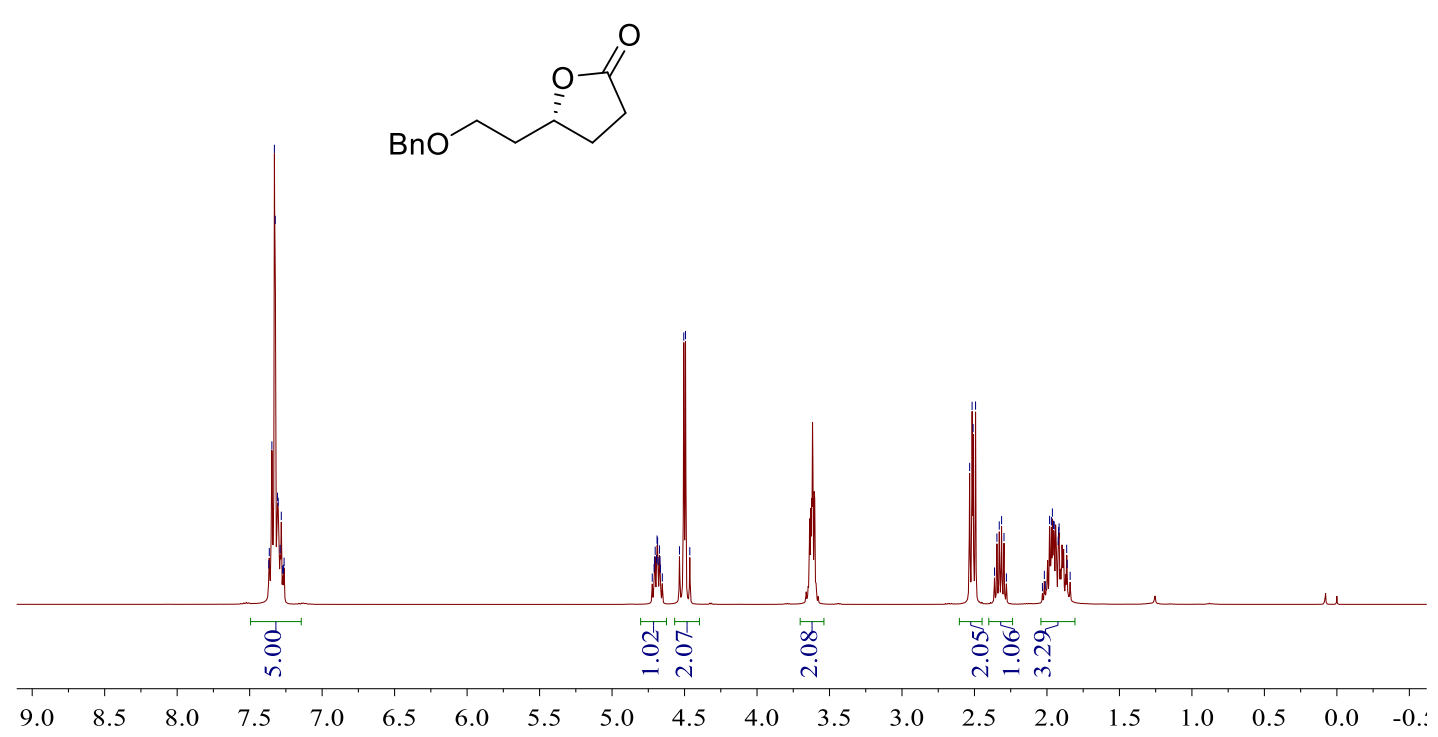

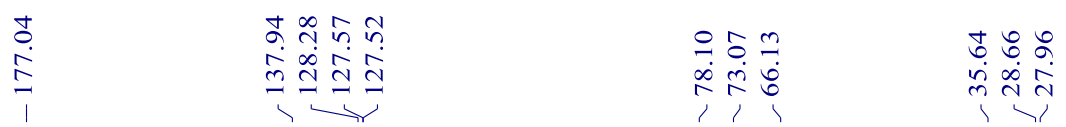

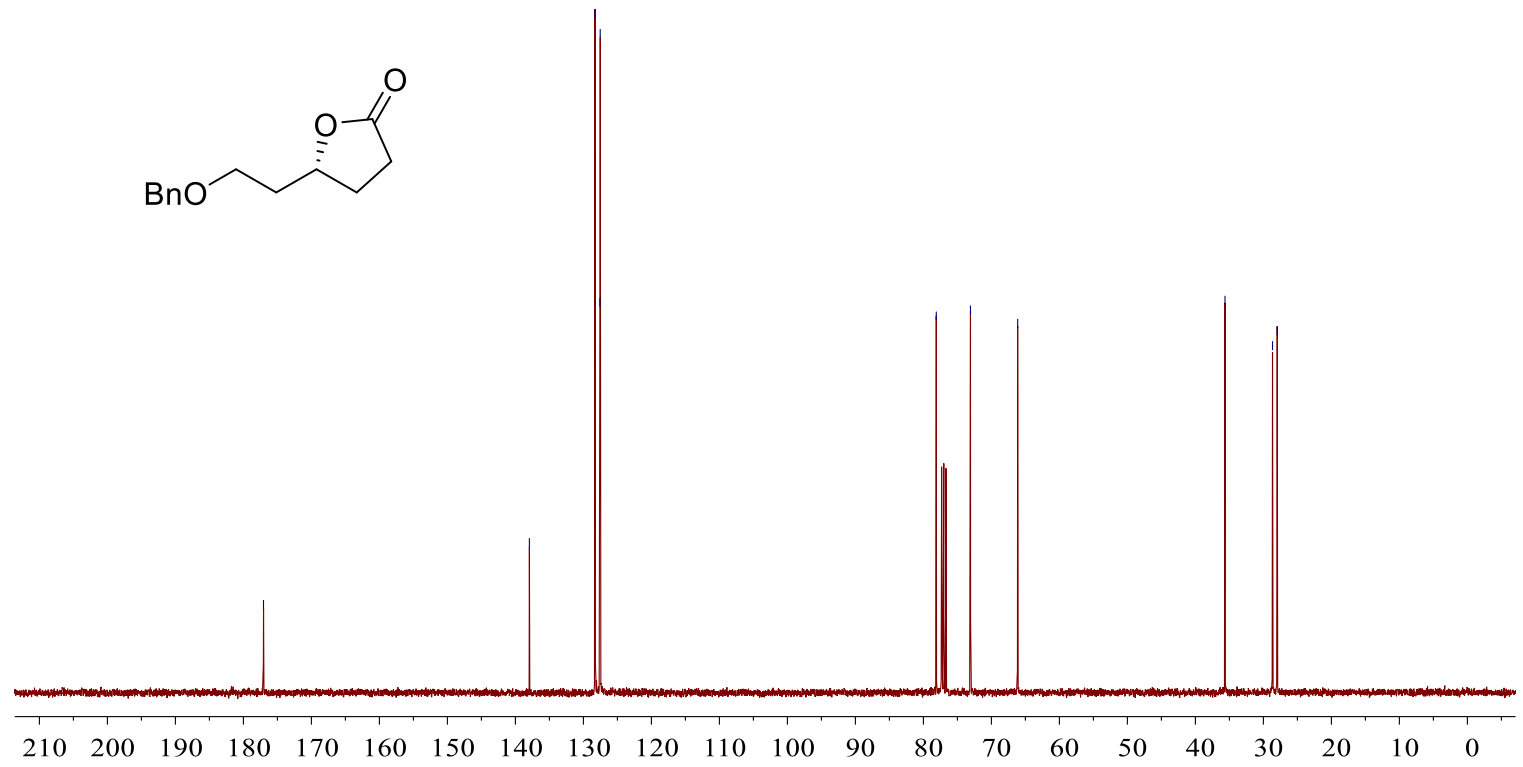




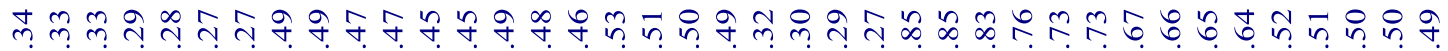

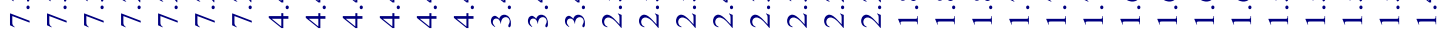
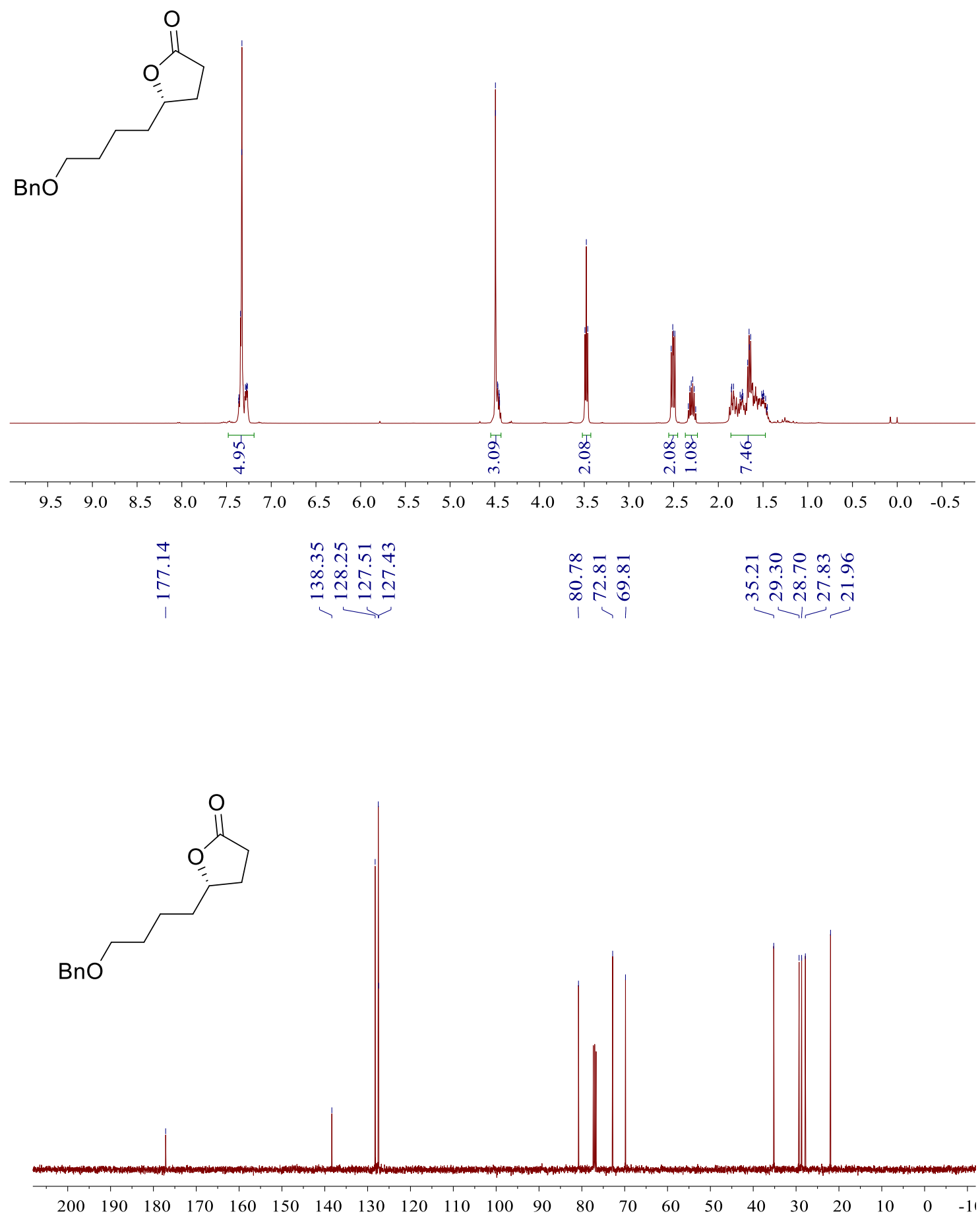

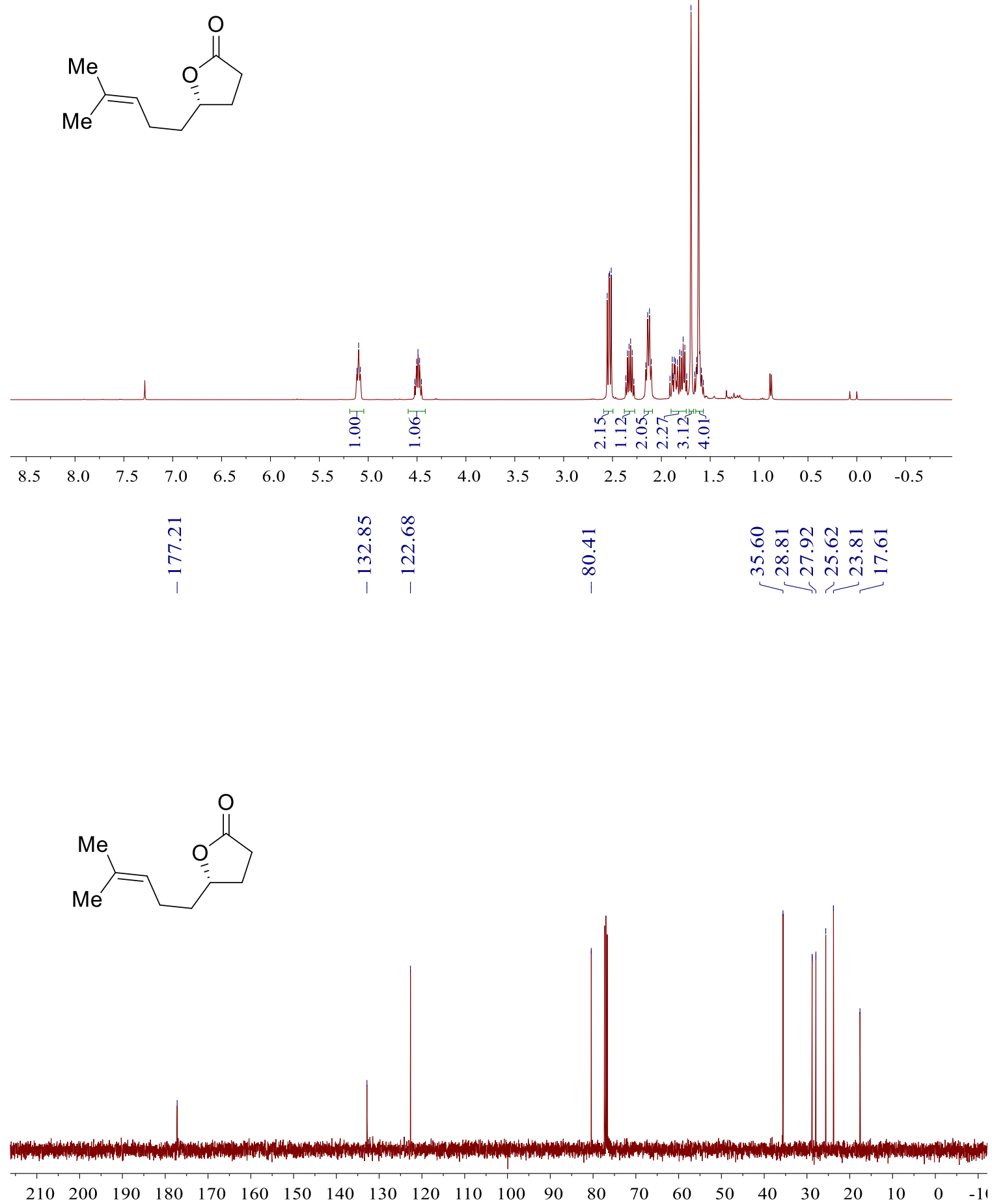


\section{1-(3-hydroxybutyl)-3-phenylurea(S6)}
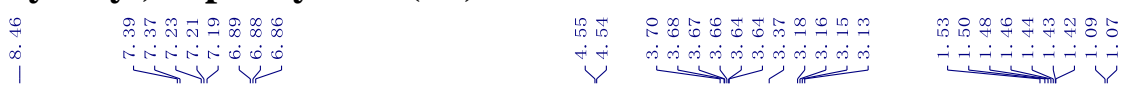

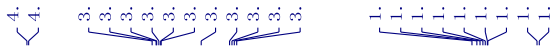<smiles>CC(O)CCNC(=O)Nc1ccccc1</smiles>

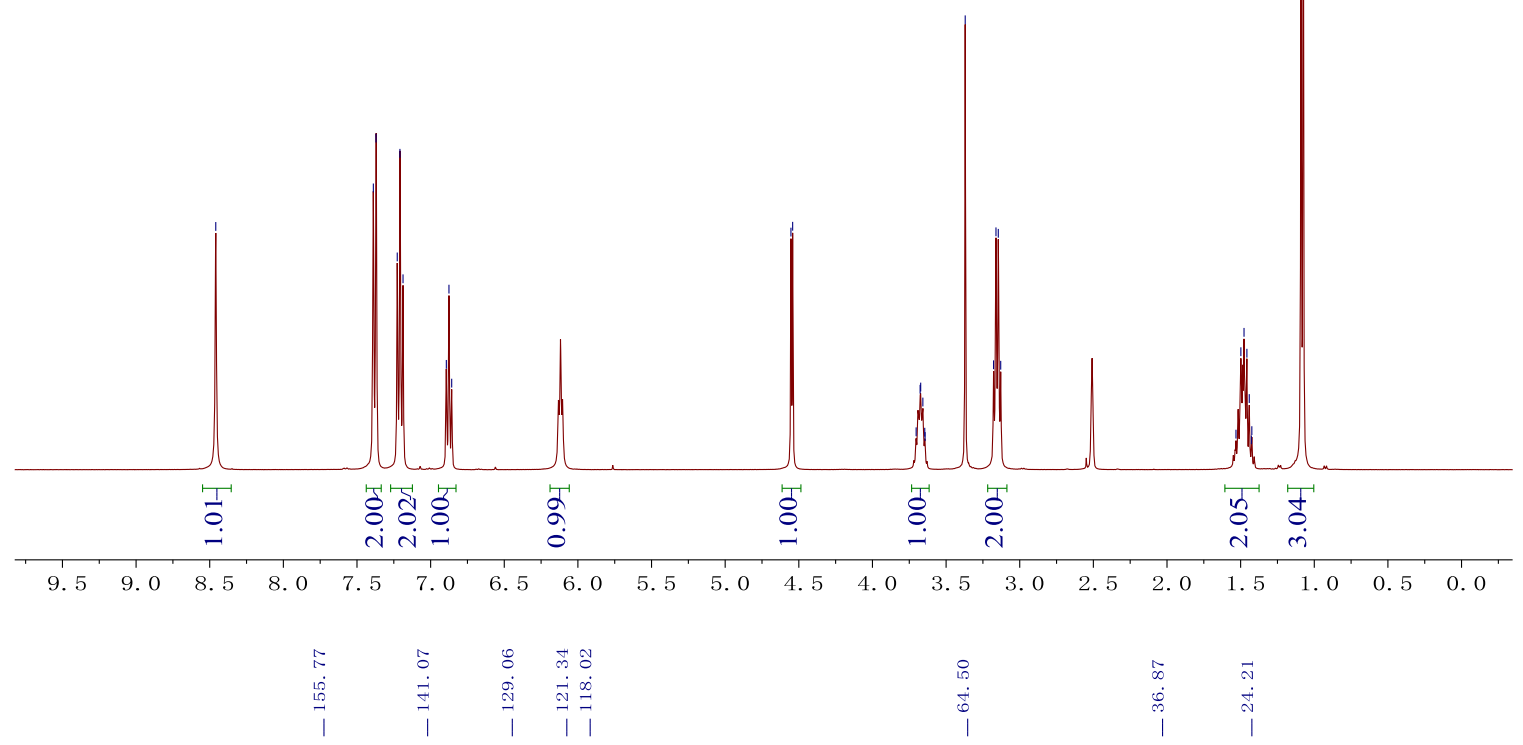<smiles>CC(O)CCNC(=O)Nc1ccccc1</smiles>

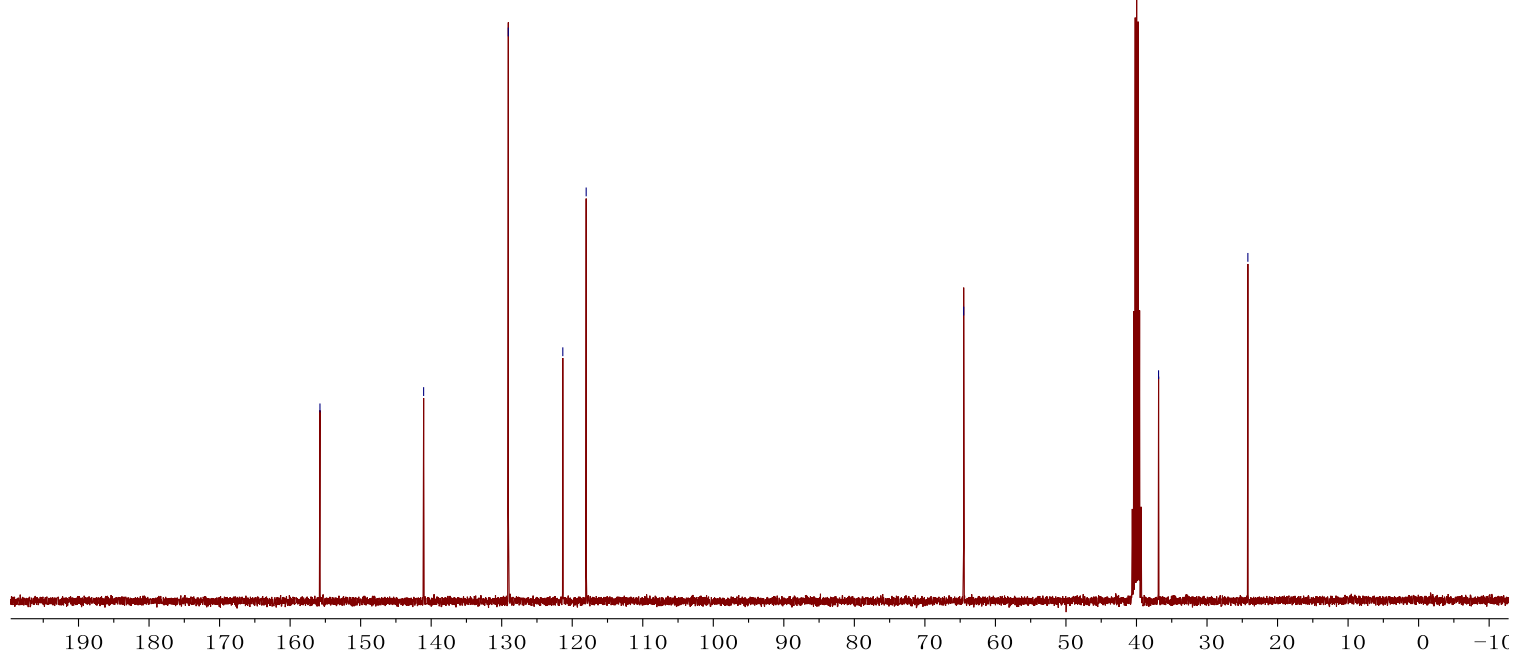




\section{GC and HPLC Charts of Hydrogenation Products}

\section{(R)-5-methyldihydrofuran-2(3H)-one (3a)}
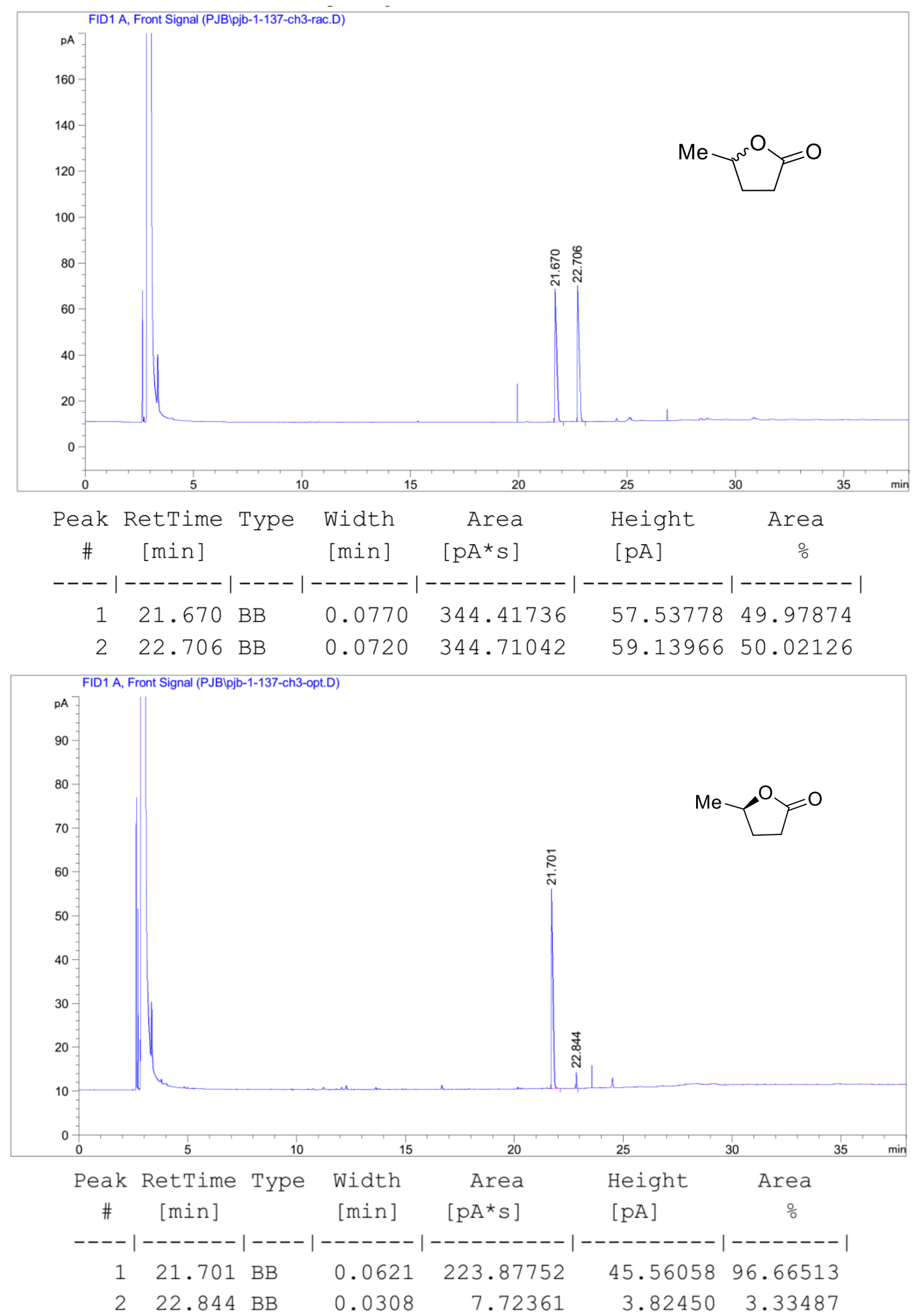
(R)-5-ethyldihydrofuran-2(3H)-one (3b)

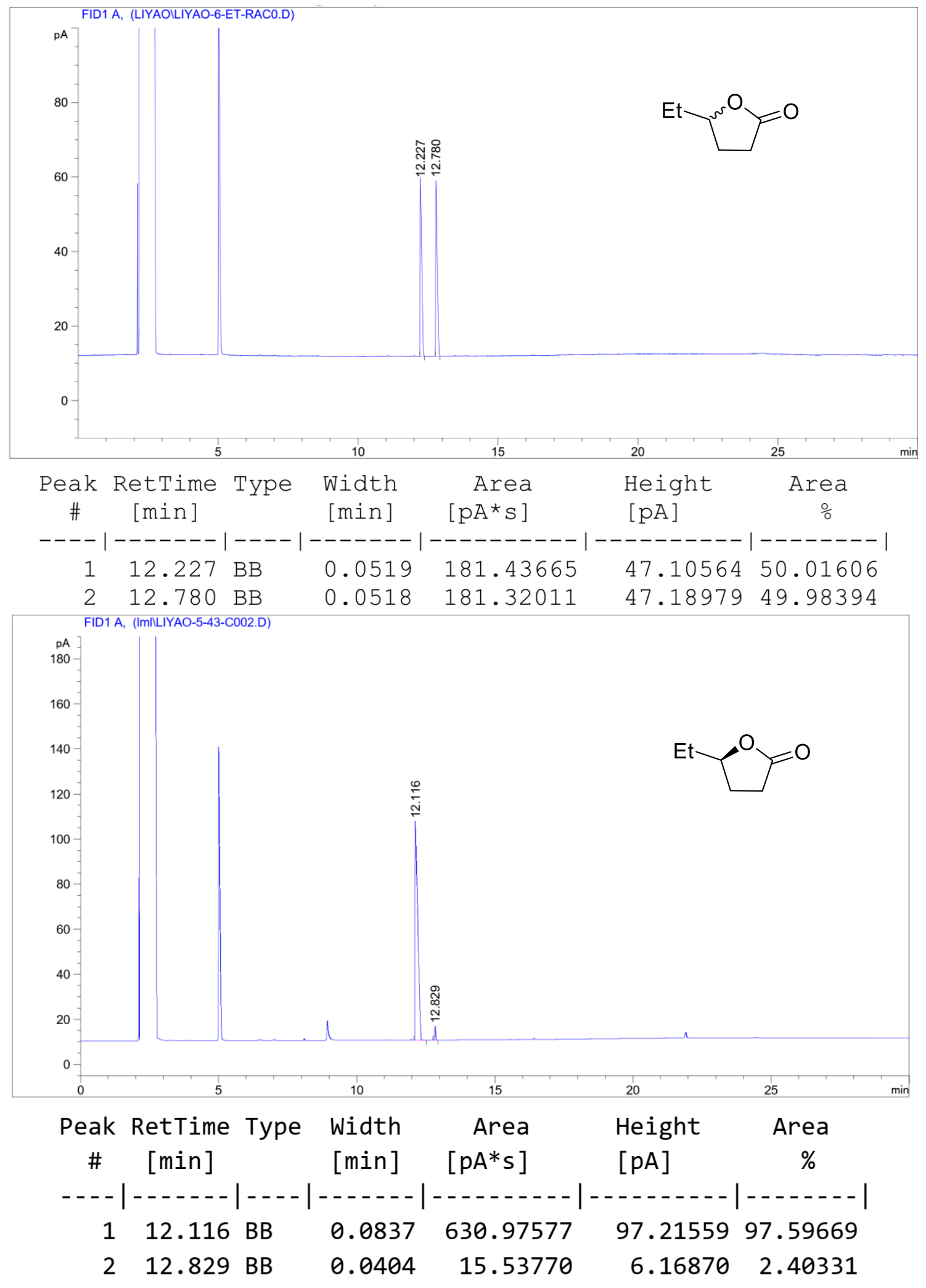




\section{(R)-5-butyldihydrofuran-2(3H)-one (3c)}

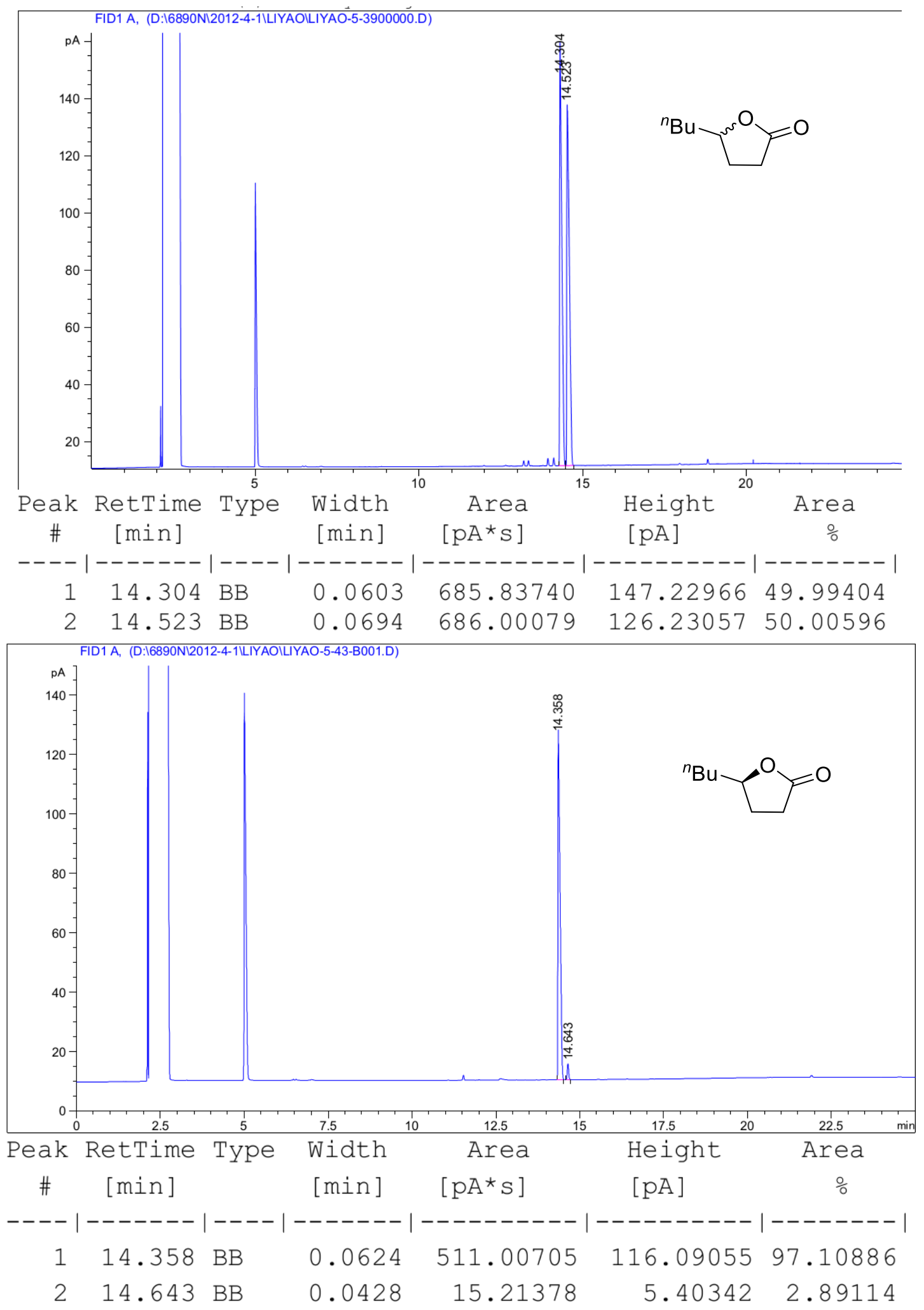


(R)-5-heptyldihydrofuran-2(3H)-one (3d)

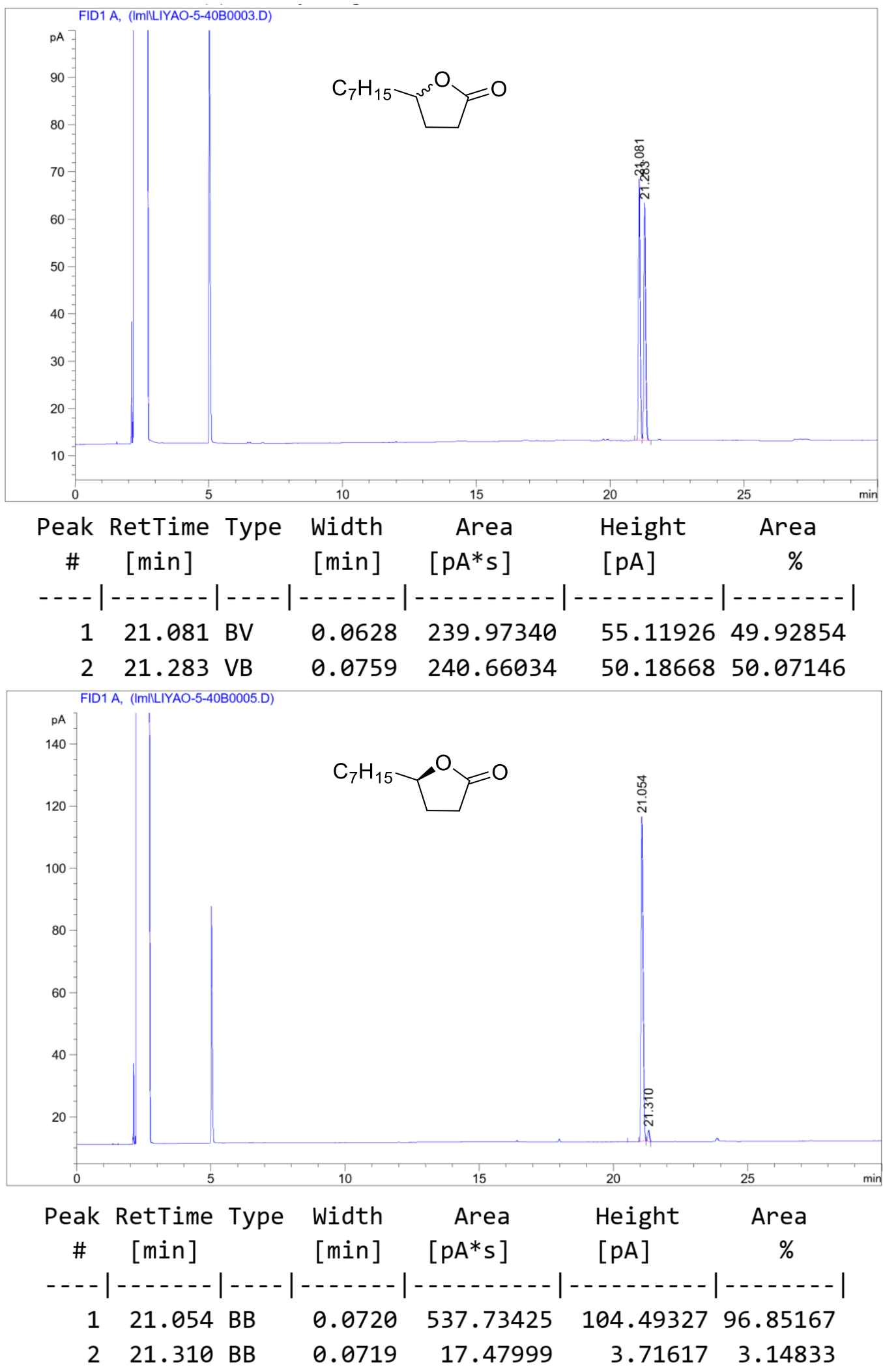


(+)-5-isobutyldihydrofuran-2(3H)-one (3e)

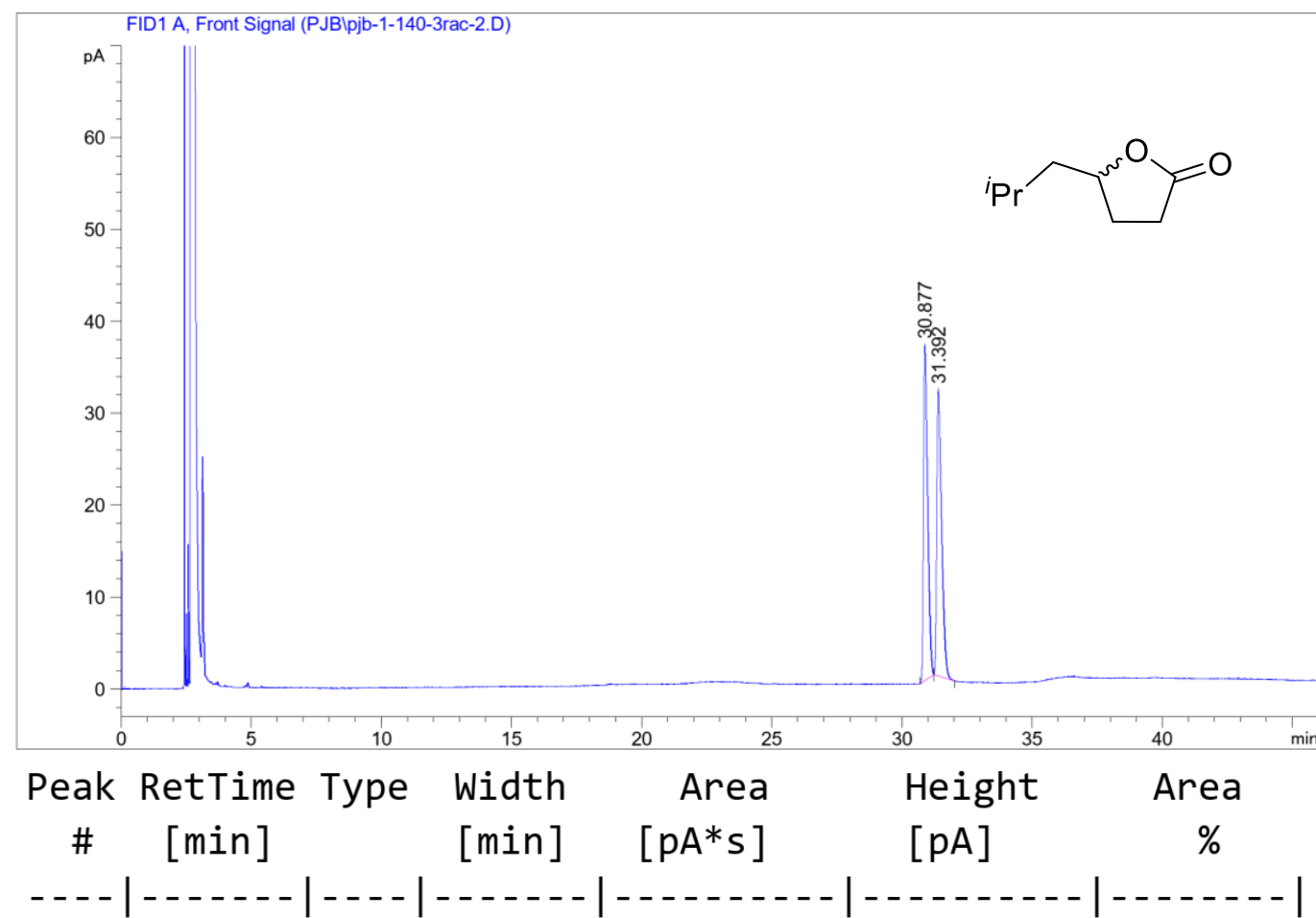

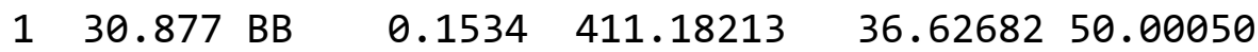

$\begin{array}{lllllll}2 & 31.392 & \text { BB } & 0.1568 & 411.17383 & 31.23999 & 49.99950\end{array}$

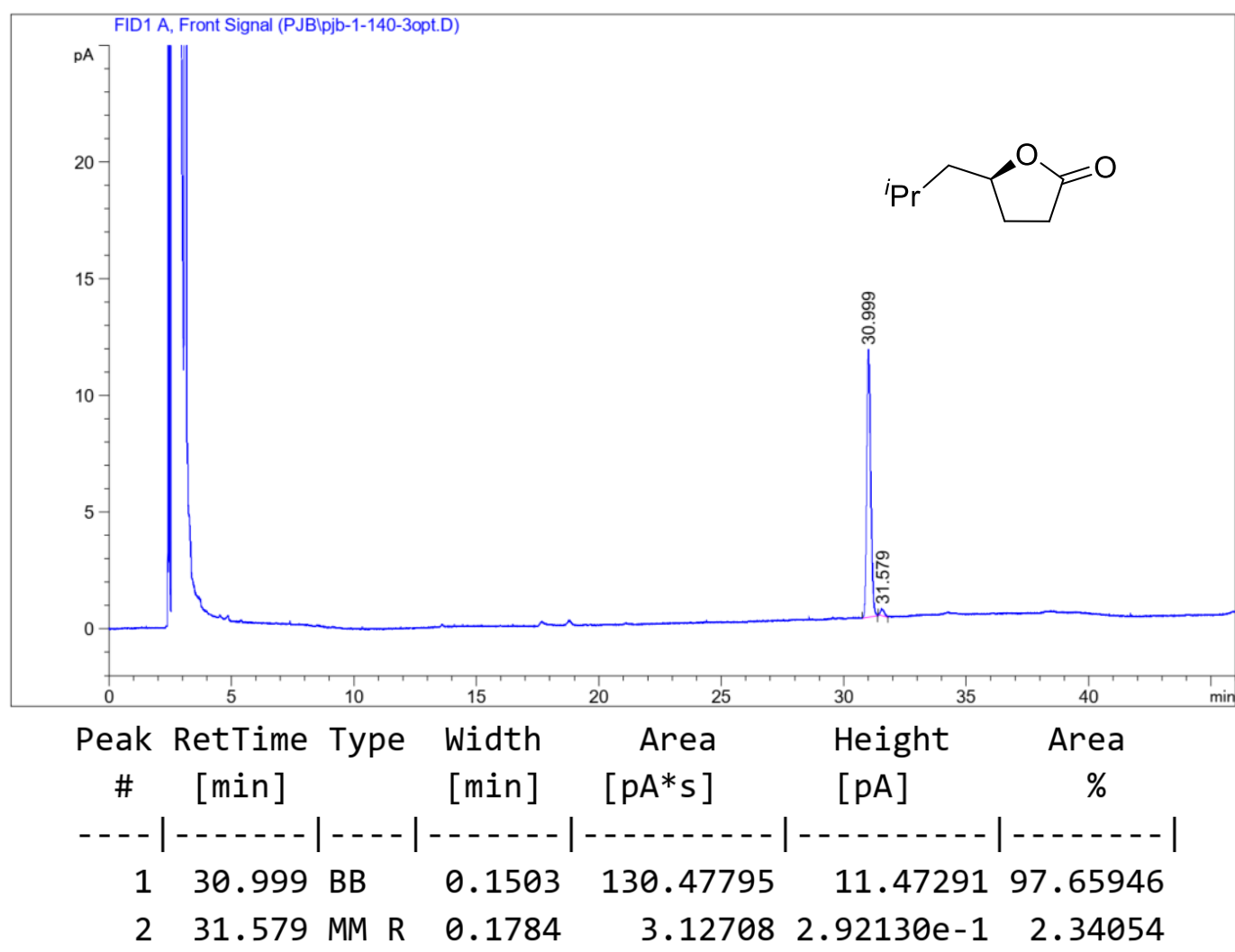




\section{(S)-5-isopropyldihydrofuran-2(3H)-one (3f)}

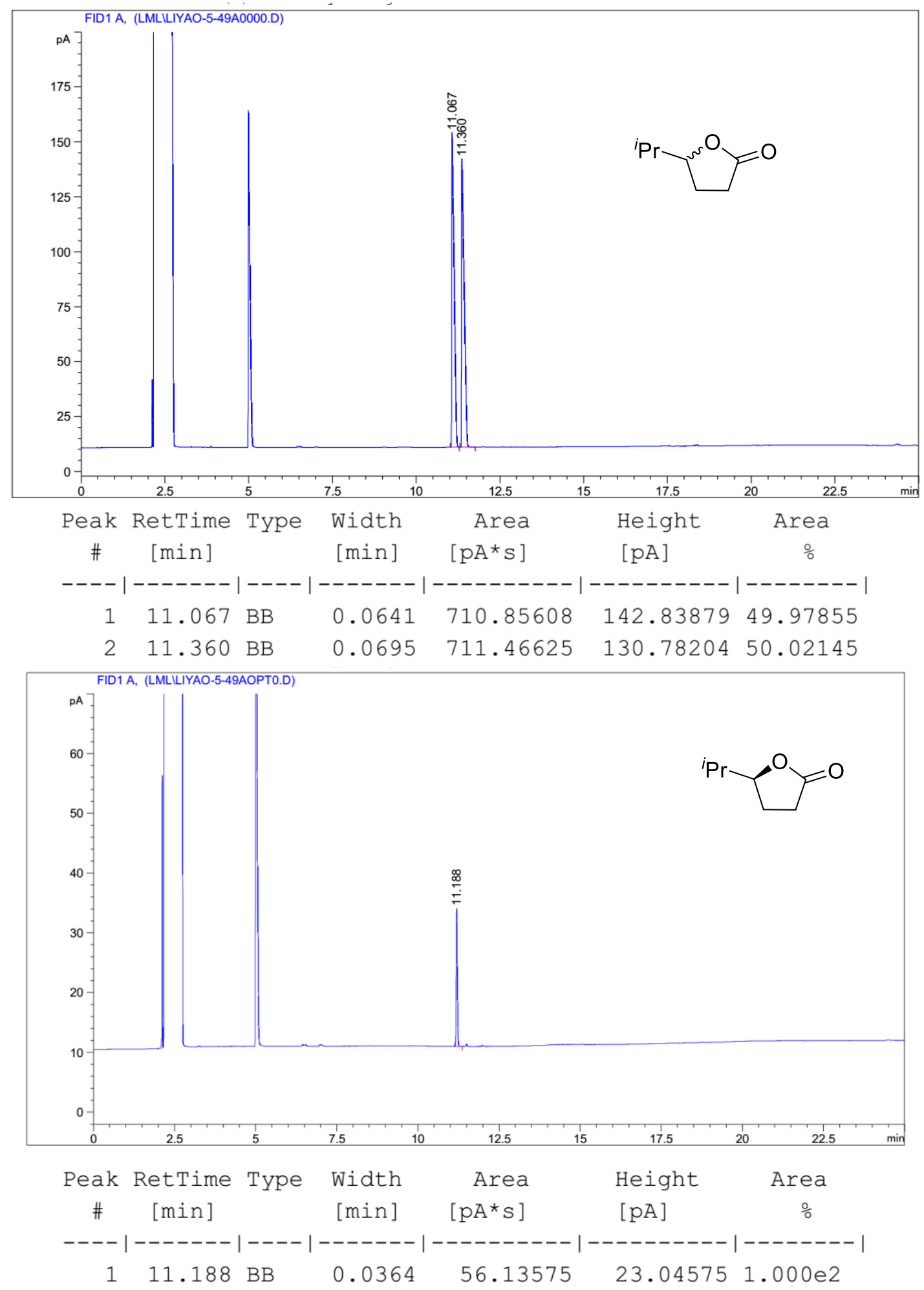




\section{(+)-5-cyclopentyldihydrofuran-2(3H)-one (3g)}
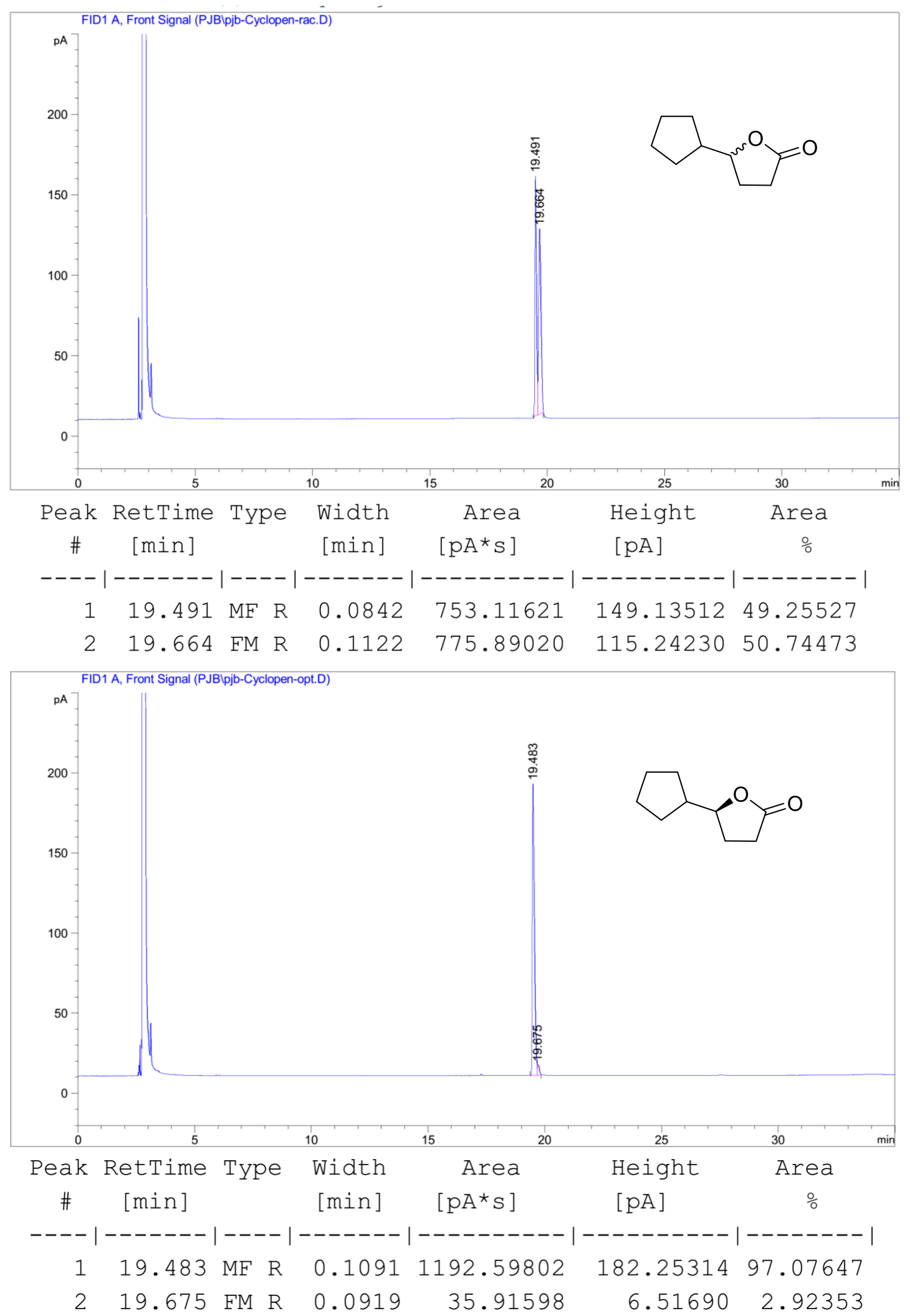
(S)-5-cyclohexyldihydrofuran-2(3H)-one (3h)

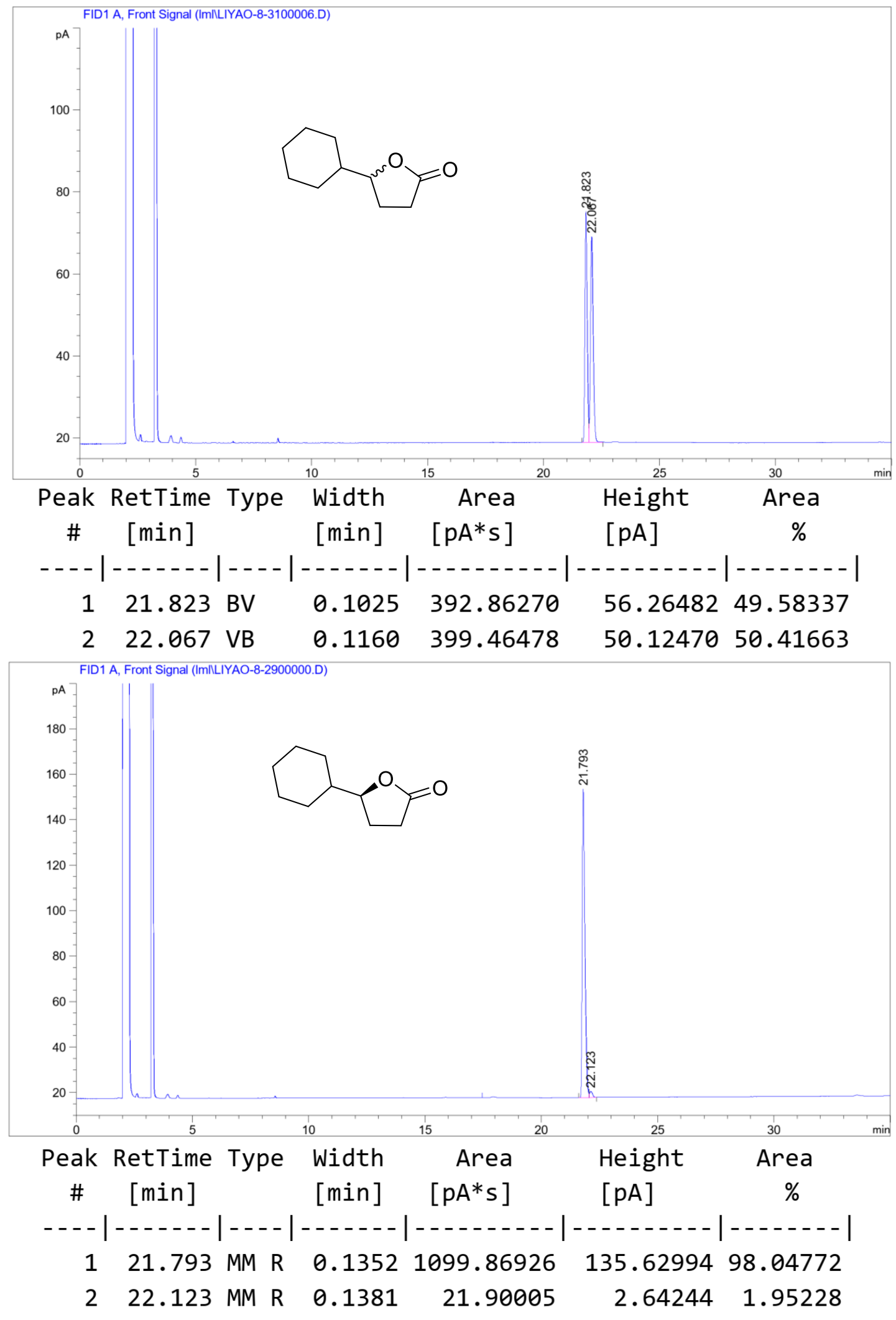




\section{(S)-5-benzyldihydrofuran-2(3H)-one (3i)}
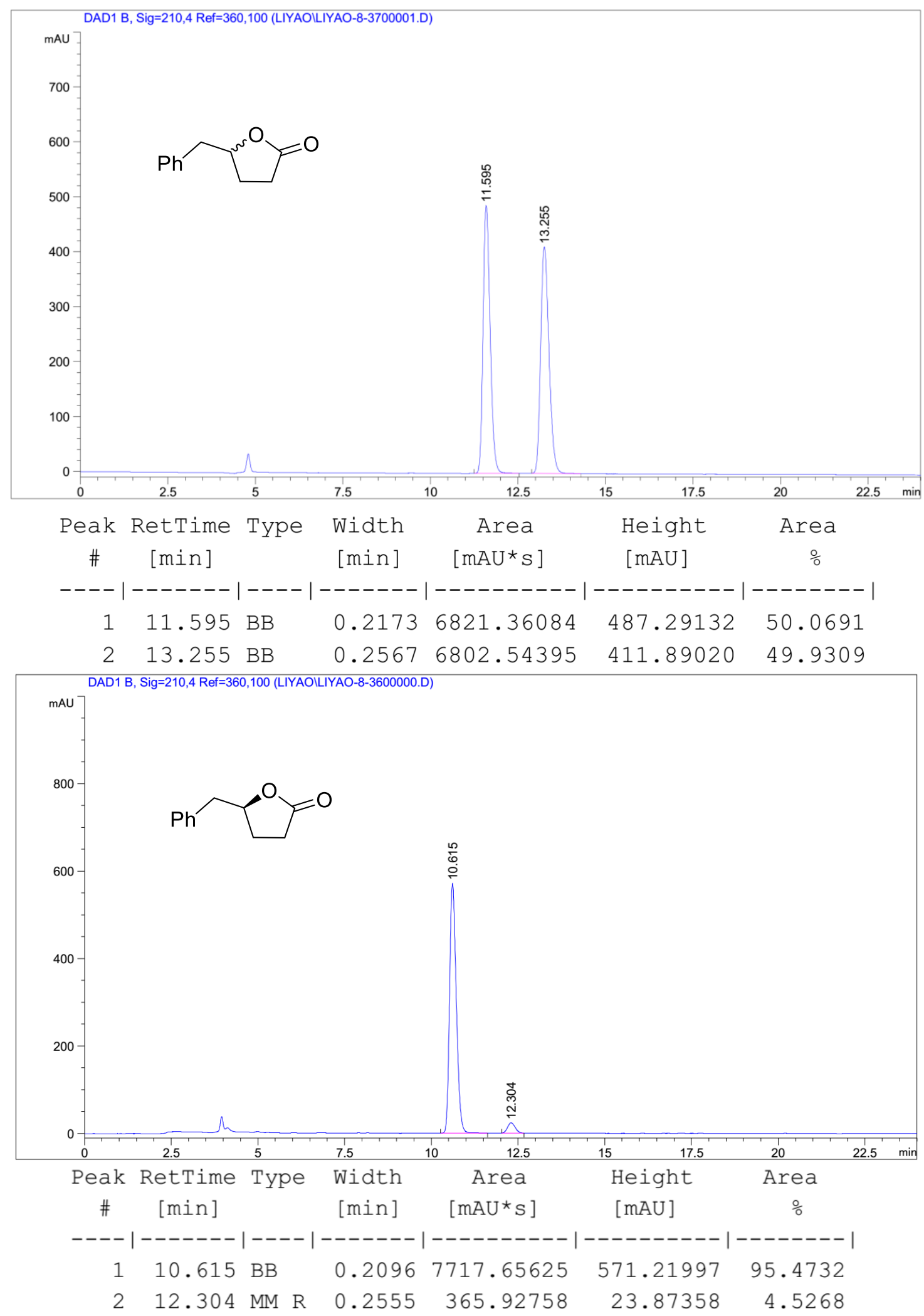
(+)-5-phenethyldihydrofuran-2(3H)-one (3j)
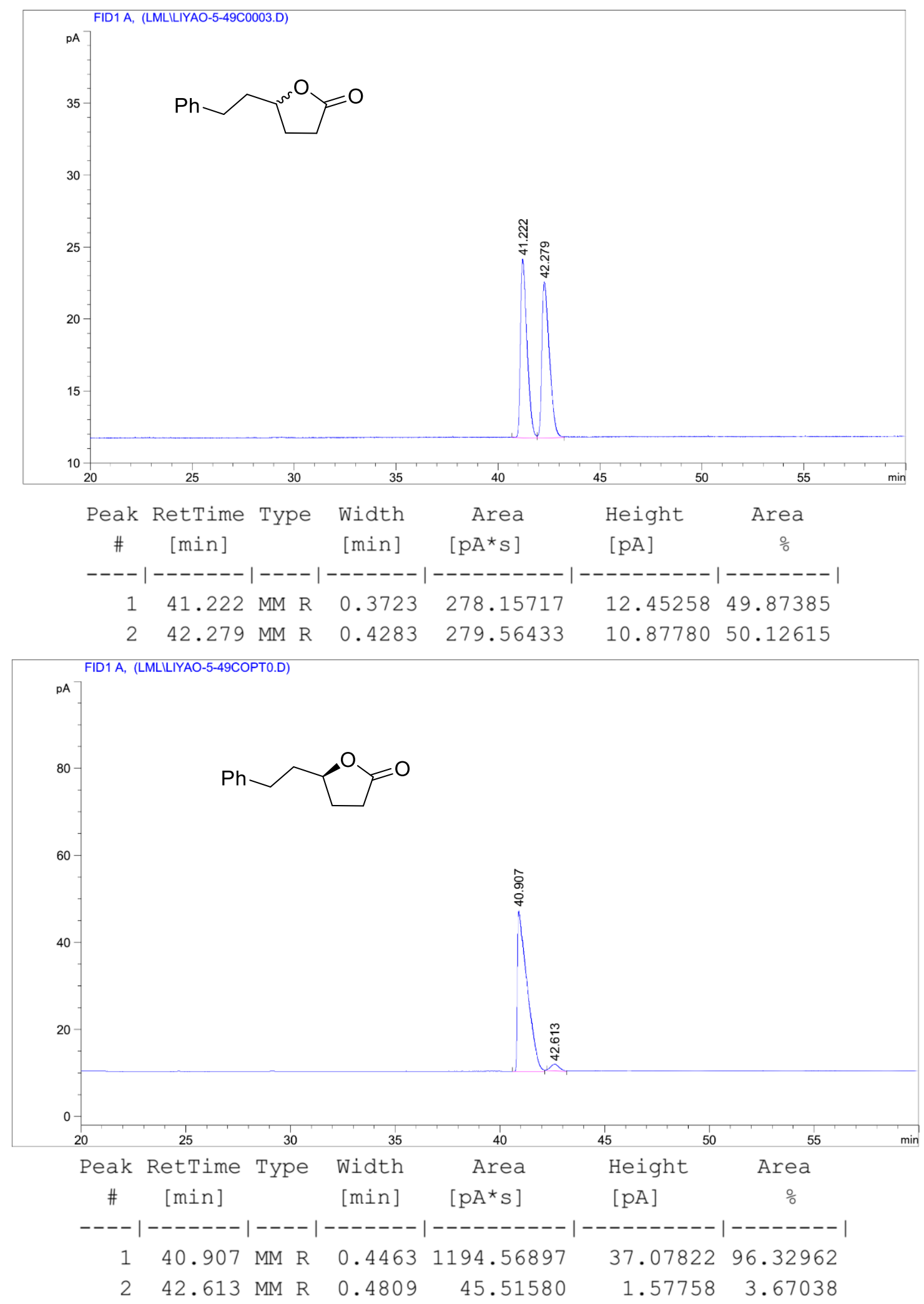


\section{(+)-5-(2-(benzyloxy)ethyl)dihydrofuran-2(3H)-one (3k)}
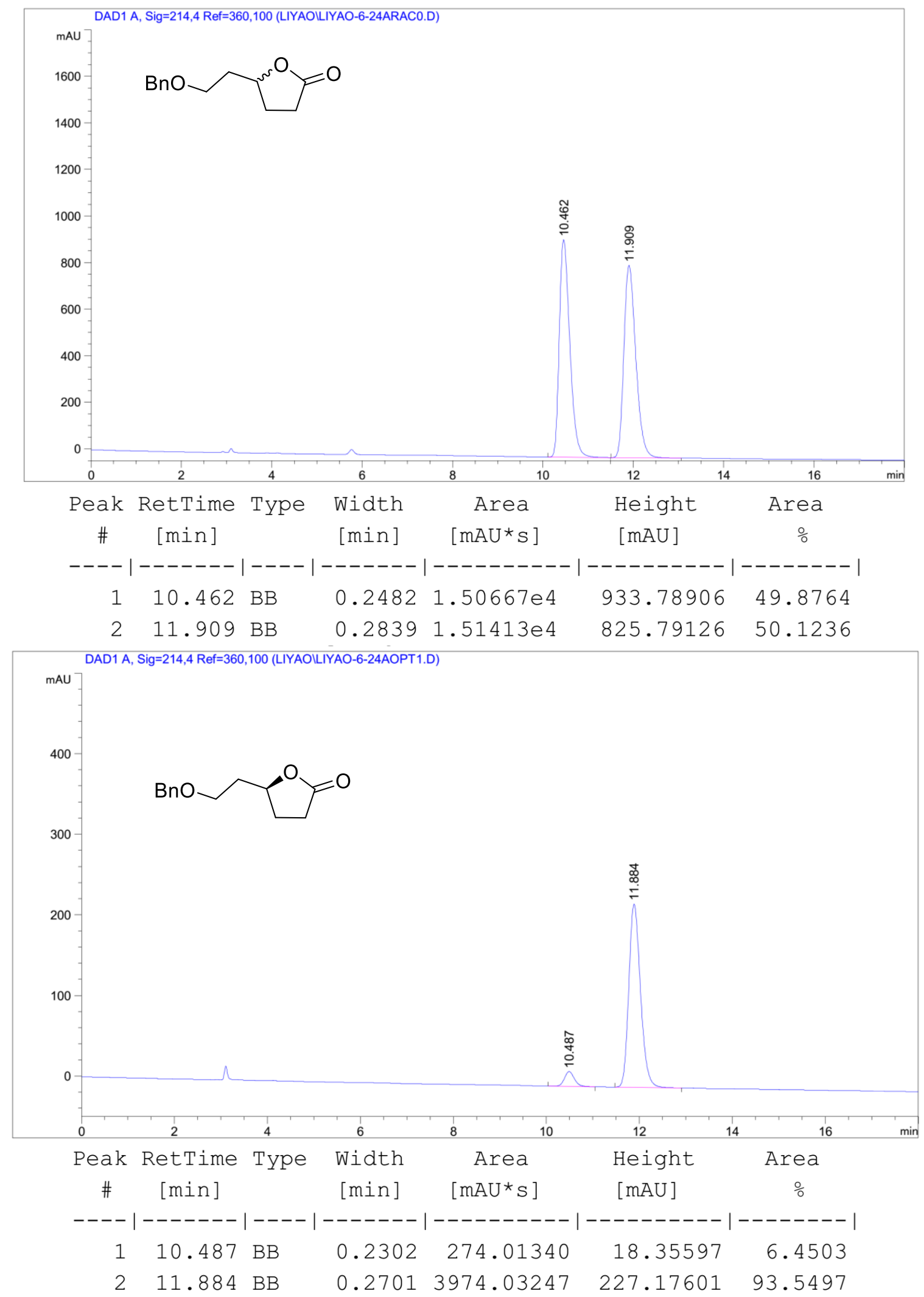
(+)-5-(4-(benzyloxy)butyl)dihydrofuran-2(3H)-one (3l)
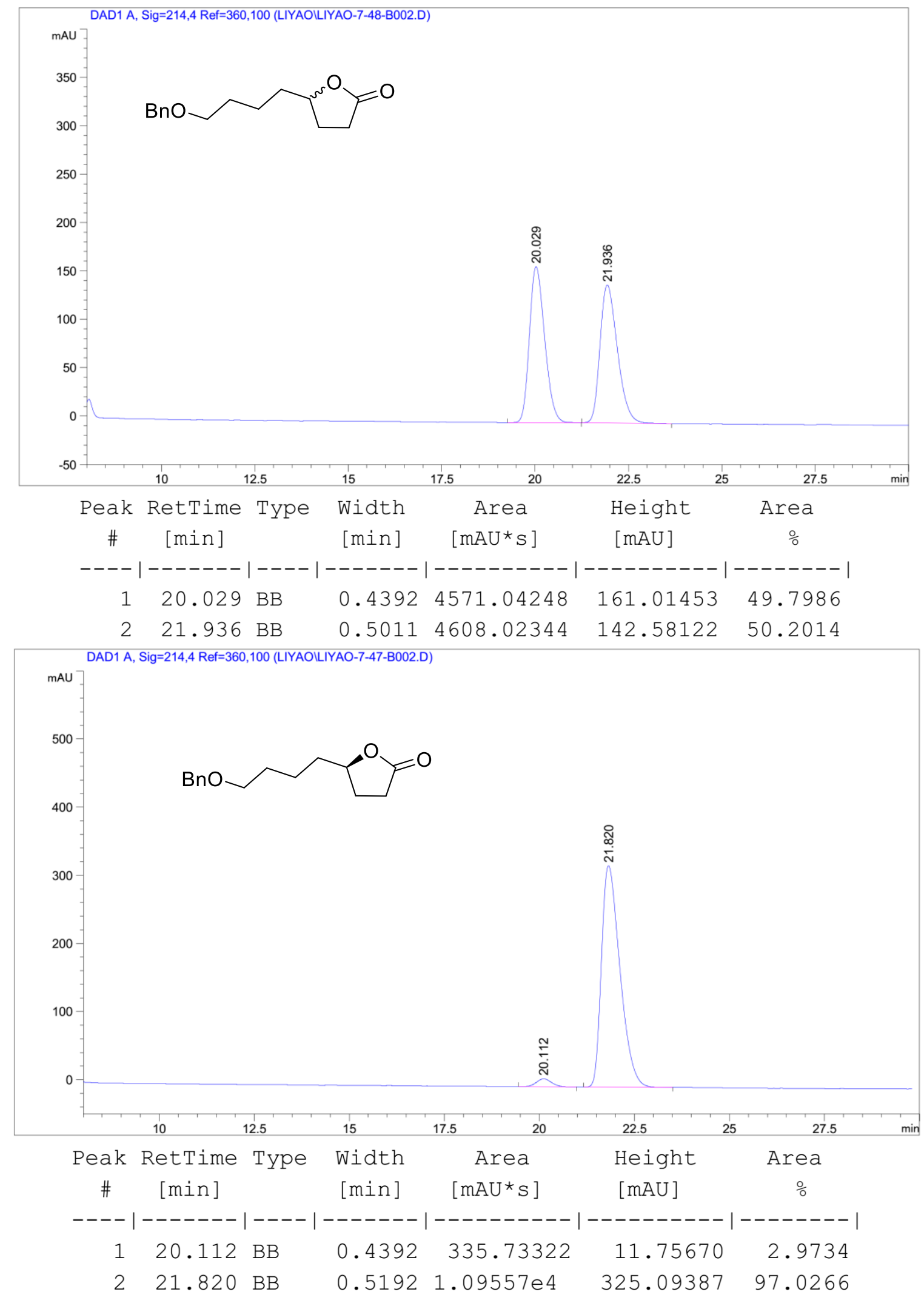
(+)-5-(4-methylpent-3-enyl)dihydrofuran-2(3H)-one (3m)
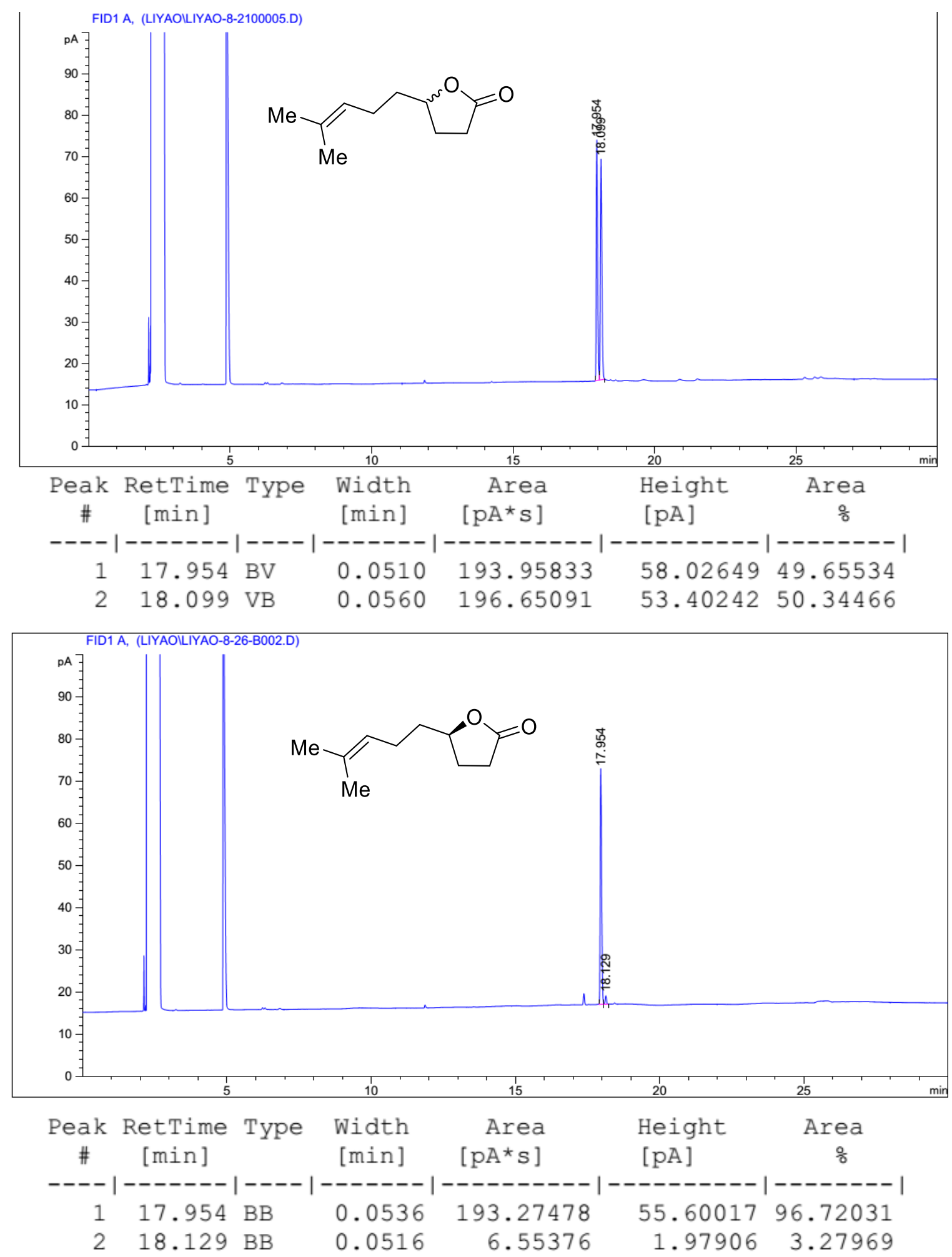
(+)-ethyl 3-(5-oxotetrahydrofuran-2-yl)propanoate (3o)
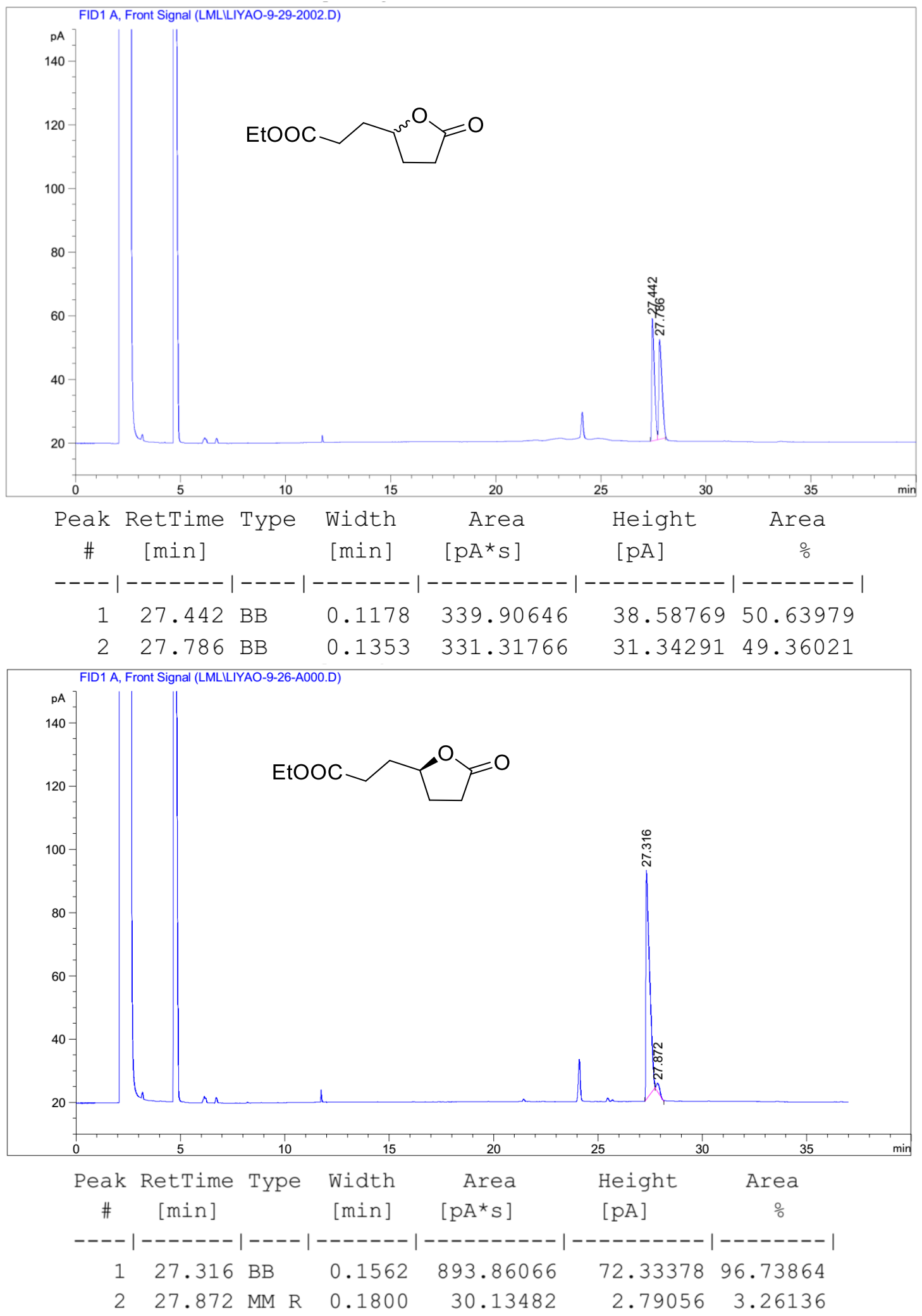


\section{(S)-5-phenyldihydrofuran-2(3H)-one (S1)}

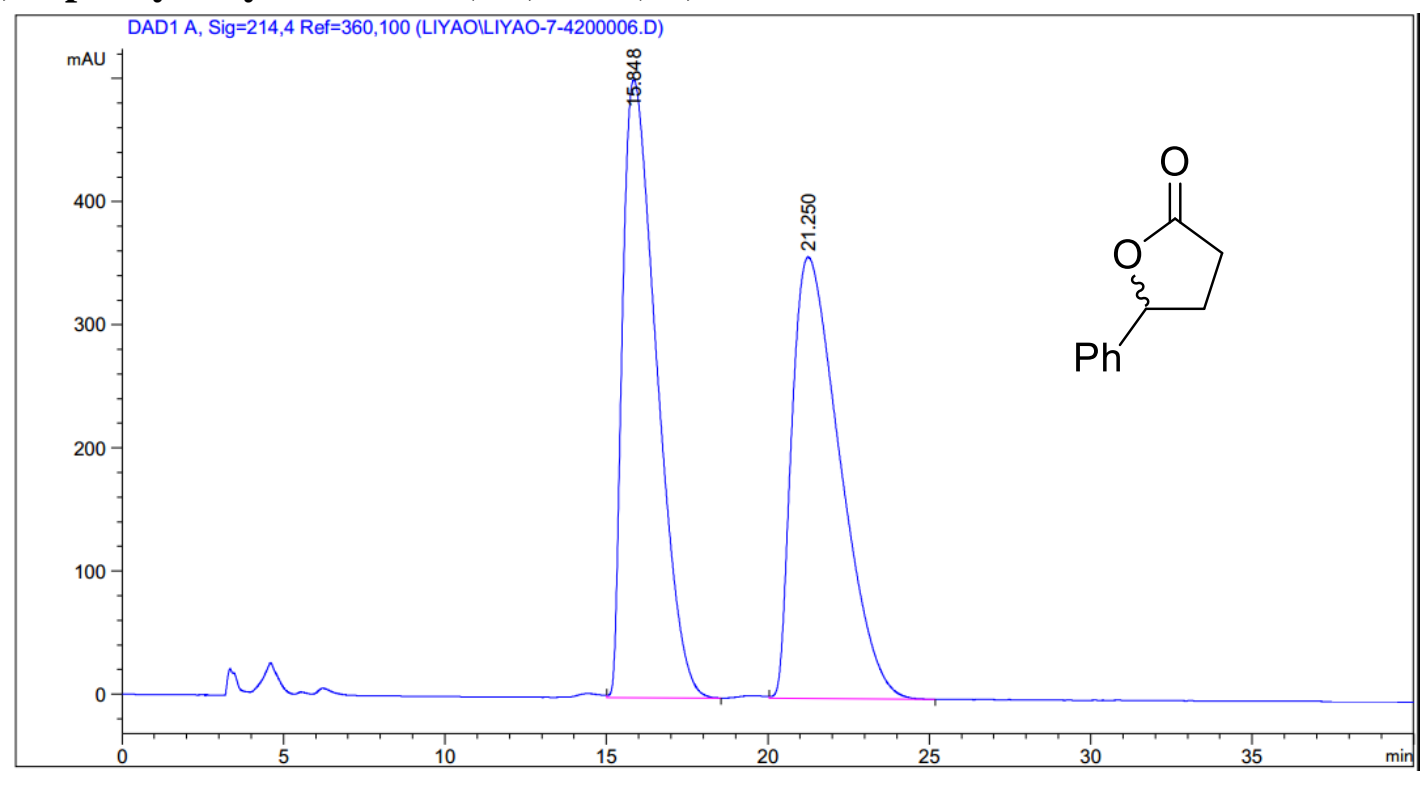

$$
\begin{aligned}
& \text { Peak RetTime Type Width Area Height Area } \\
& \text { \# }[\mathrm{min}] \quad[\mathrm{min}] \quad\left[\mathrm{mAU}^{*} \mathrm{~s}\right] \quad[\mathrm{mAU}] \quad \text { \% } \\
& \text {----|-------|----|-------|----------|----------|--------| } \\
& \begin{array}{llllll}
1 & 15.848 \text { VB } & 1.1404 & 3.66714 \mathrm{e} 4 & 501.89868 & 49.8201
\end{array} \\
& \begin{array}{llllll}
2 & 21.250 \mathrm{VB} & 1.6097 & 3.69362 \mathrm{e} & 358.58548 & 50.1799
\end{array}
\end{aligned}
$$

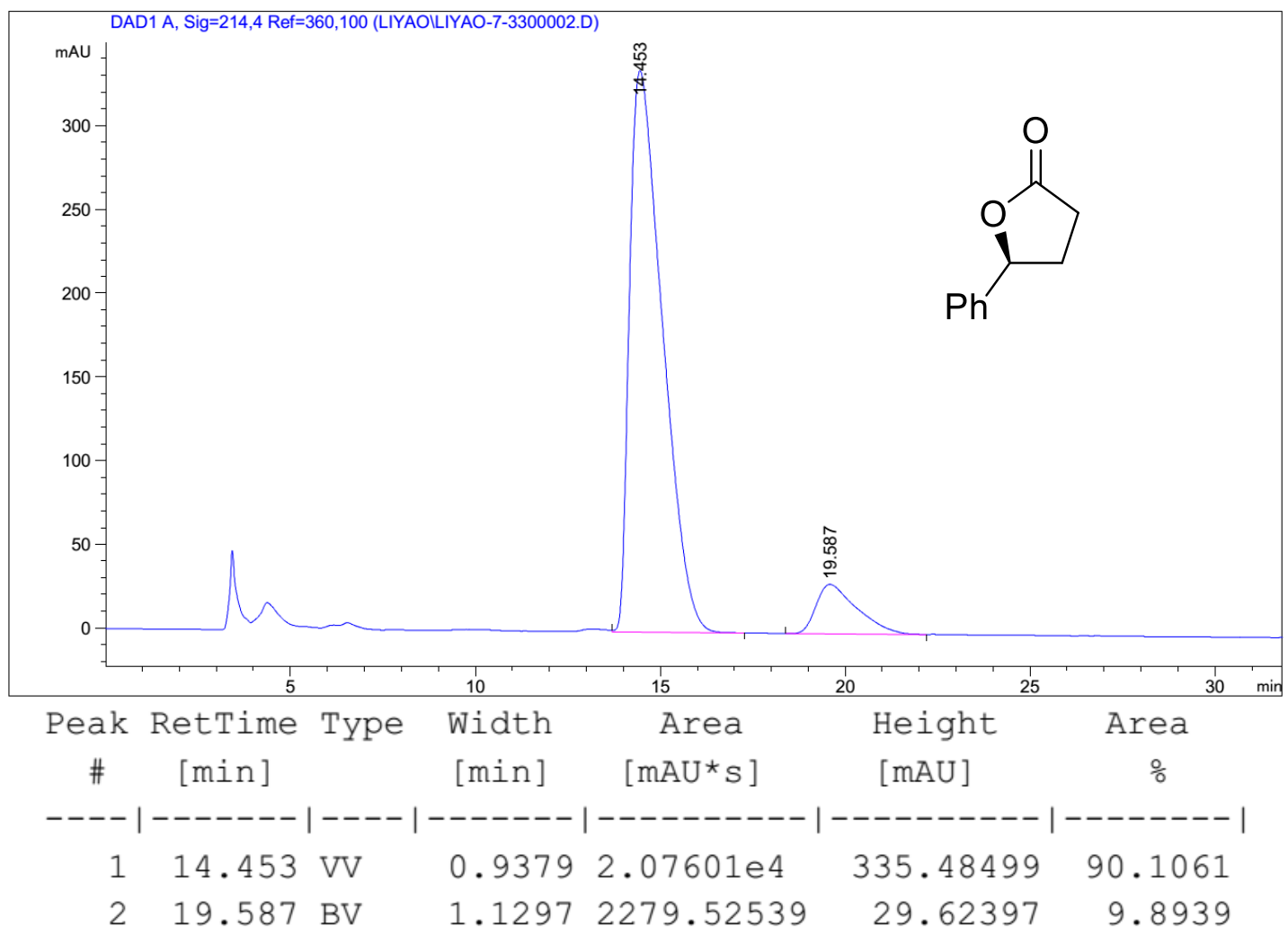




\section{4-hydroxy-N-phenylpentanamide(S3)}

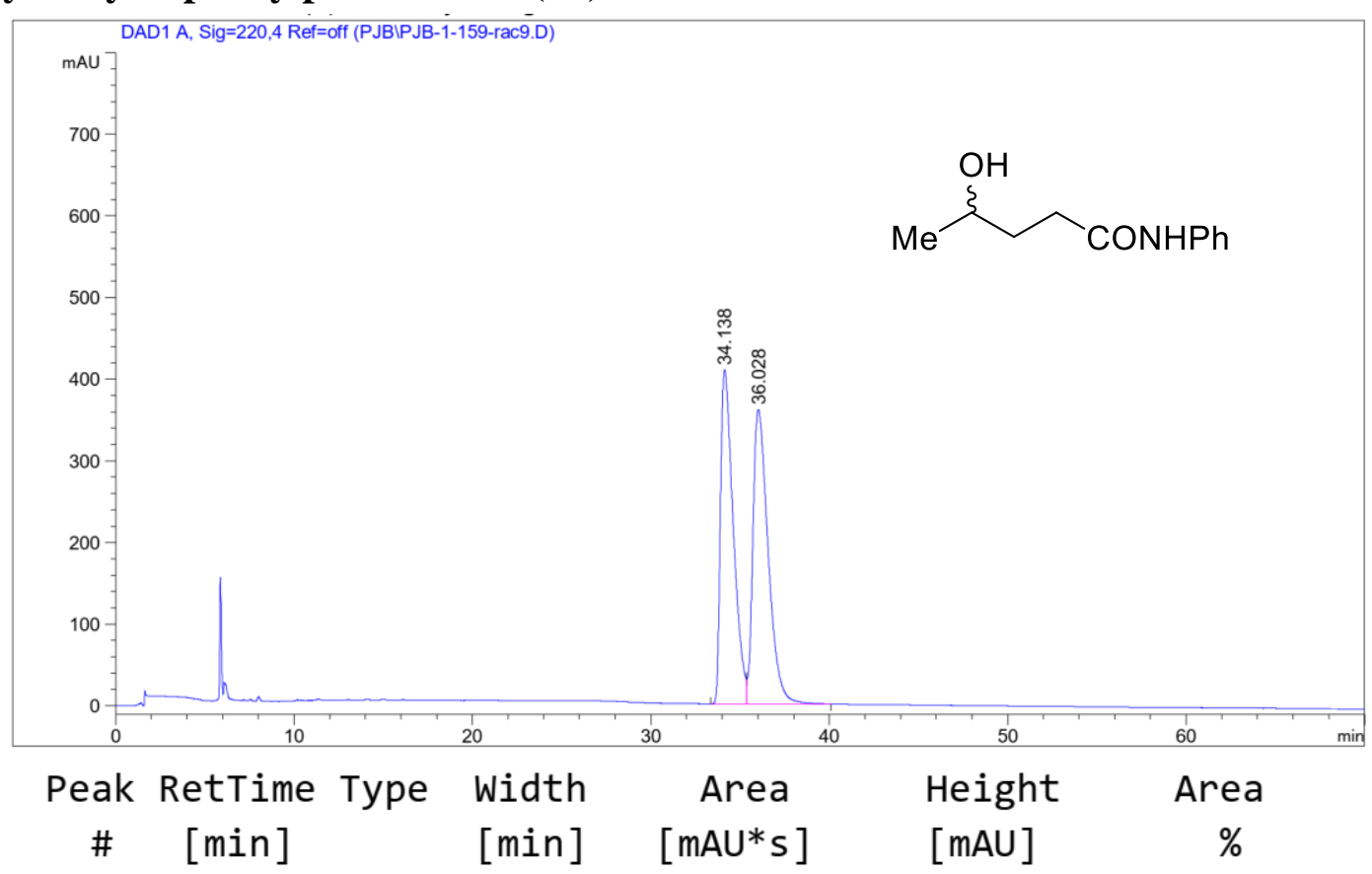

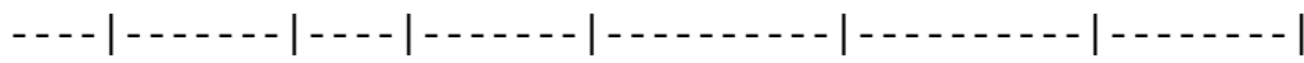

$\begin{array}{lllllll}1 & 34.138 & \text { BV } & 0.7504 & 2.03479 \mathrm{e} 4 & 409.04752 & 48.7089\end{array}$

$\begin{array}{llllll}2 & 36.028 \text { VB } & 0.9159 & 2.14266 \mathrm{e} & 360.27097 & 51.2911\end{array}$

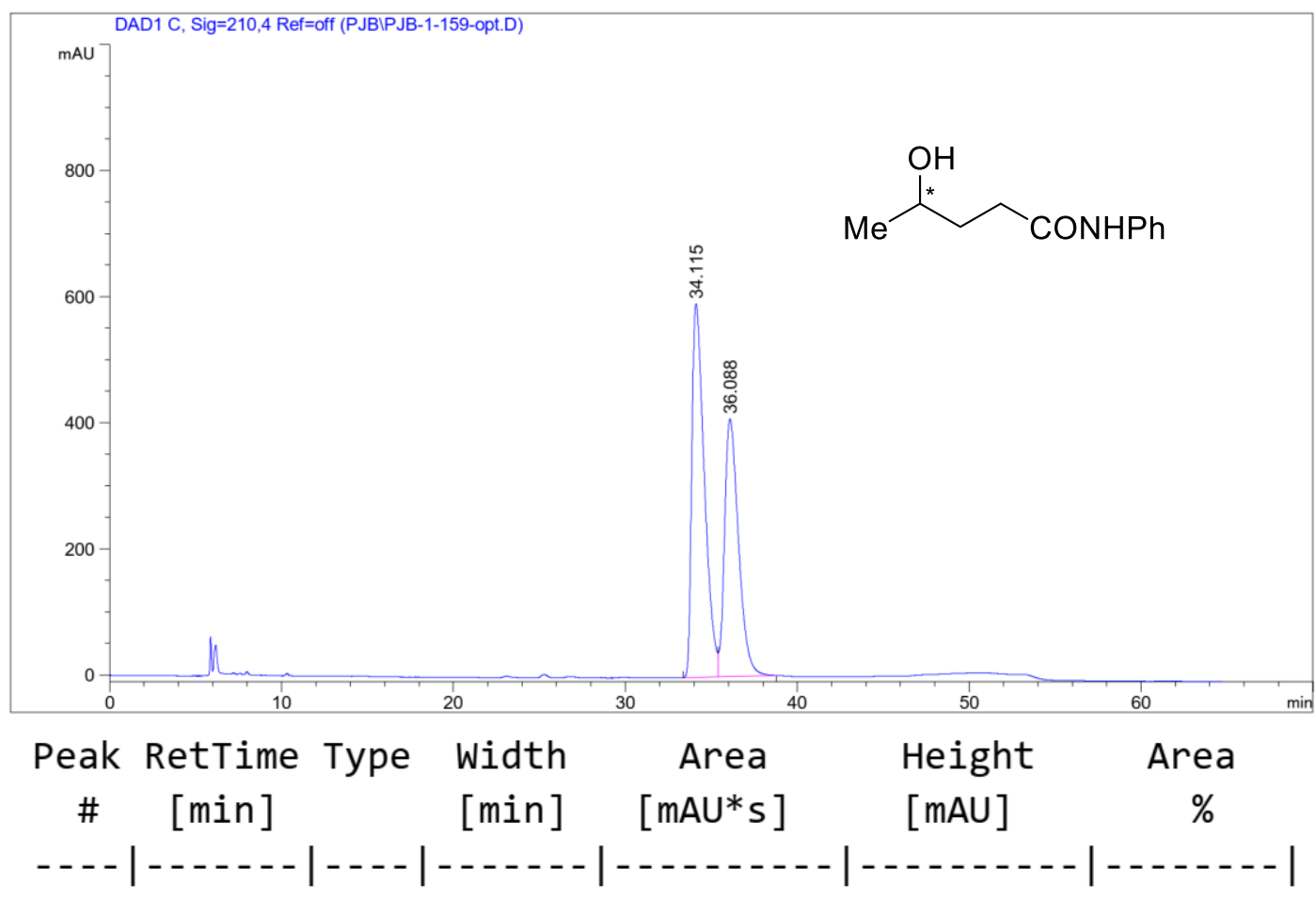

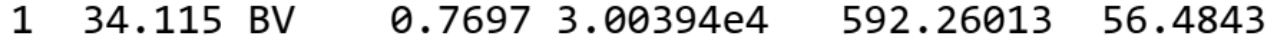

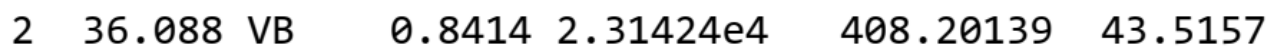




\section{1-(3-hydroxybutyl)-3-phenylurea(S6)}
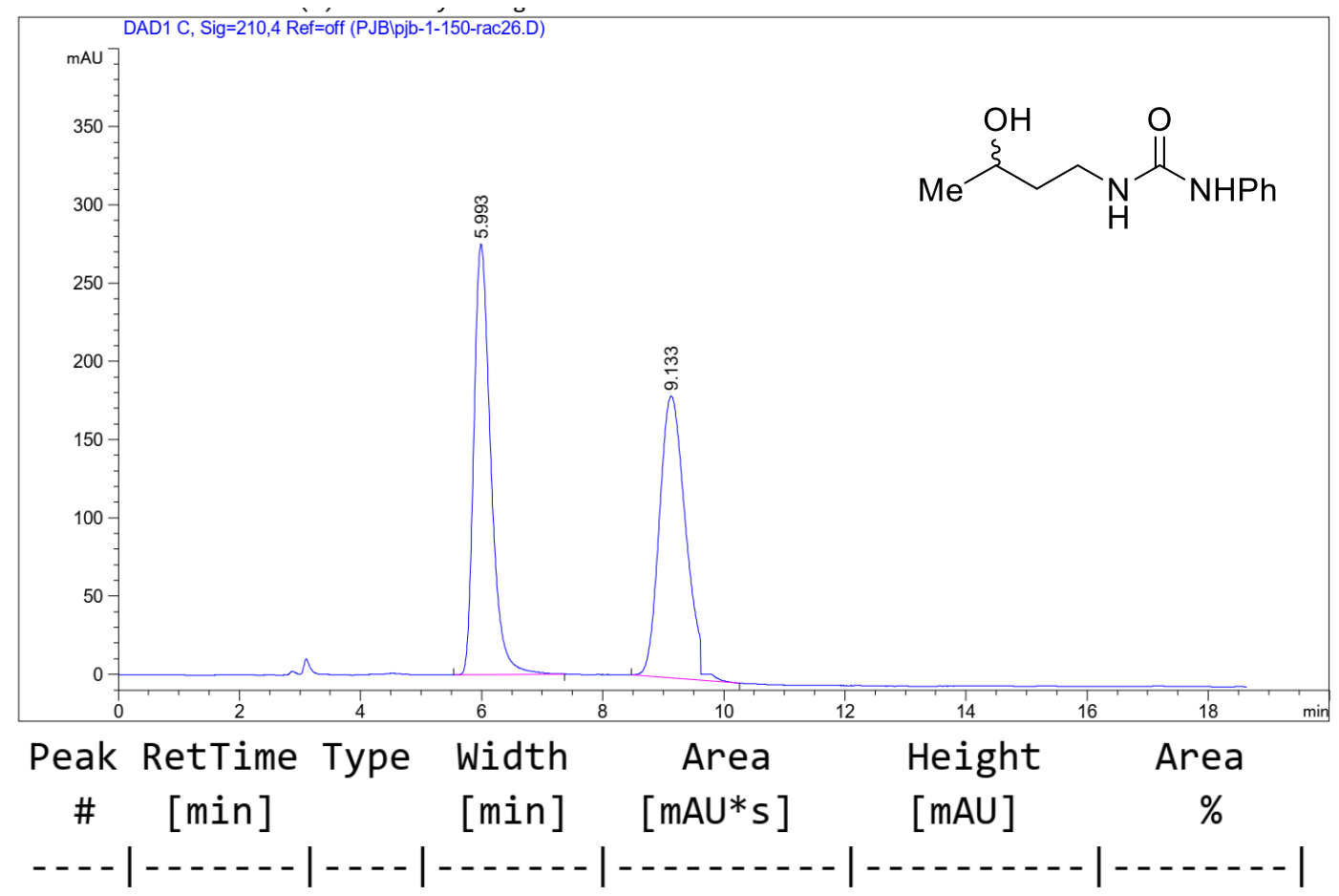

$\begin{array}{lllllll}1 & 5.993 & \text { BB } & 0.2981 & 5334.01367 & 275.26941 & 49.7169\end{array}$

$\begin{array}{lllllll}2 & 9.133 & \text { BB } & 0.4594 & 5394.76416 & 180.12770 & 50.2831\end{array}$

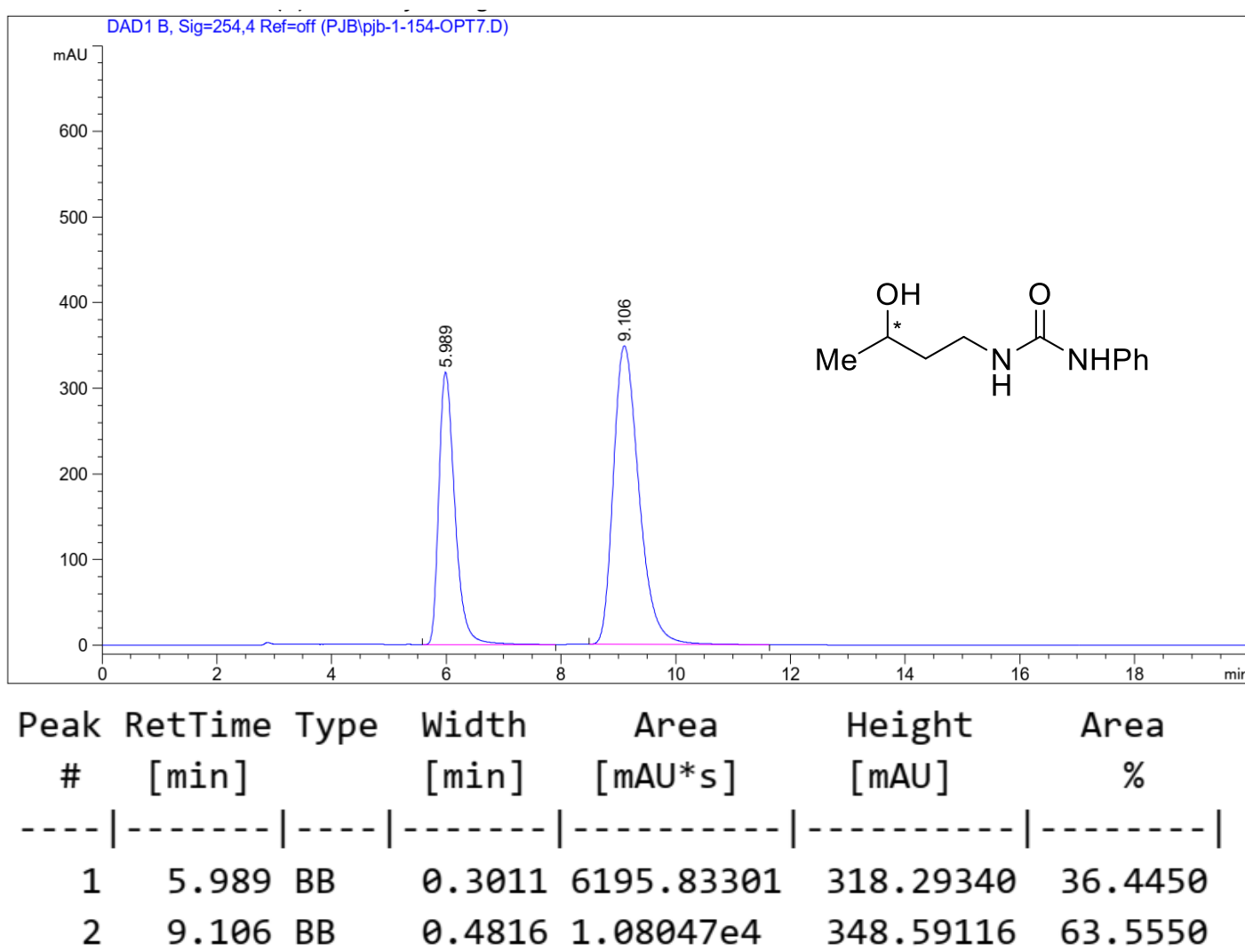




\section{Computational Data}

\section{DFT Methods}

All DFT calculations have been carried out using the Gaussian 09 program package ${ }^{[30]}$. The B3LYP ${ }^{[31-33]}$ method with def2-SVP ${ }^{[34-35]}$ basis set of Ahlrichs and coworkers has been selected for geometry optimizations and calculation of Gibbs energy corrections at $338 \mathrm{~K}$ temperature and $6 \mathrm{~atm}$. Final energies were retrieved from single-point calculations at the B3LYP level of theory with def2-TZVPP in solution using the SMD ${ }^{[36]}$ model with $\mathrm{MeOH}$ as solvent, including the D3 dispersion correction scheme developed by Grimme ${ }^{[37]}$. Some complexes were treated with charge +1 and in the single state. No symmetry constraints were applied. Reaction paths were traced by the intrinsic reaction coordinate method for all transition states. All energetics reported throughout the text are in $\mathrm{kcal} / \mathrm{mol}$. Structures were generated using CYLview ${ }^{[38]}$.

\section{The H-H metathesis process involving carboxyl oxygen}
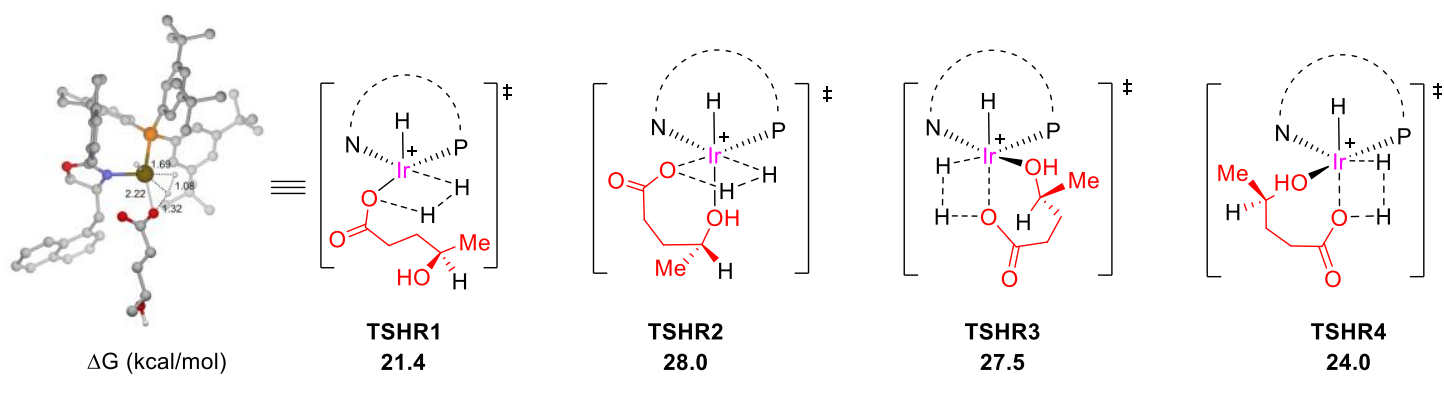

B3LYP-D3/DEF2TZVPP(SMD, MeOH) // B3LYP/DEF2SVP 
Table S1. Calculated Gibbs energy profile for the compounds

\begin{tabular}{|c|c|c|c|c|}
\hline & $\begin{array}{c}\text { Gibbs energy } \\
\text { corrections }\end{array}$ & $\begin{array}{c}\text { Single-point } \\
\text { energies }\end{array}$ & Gibbs energy & $\Delta \mathbf{G}$ \\
\hline IntAR & 1.11849400 & -3288.30408402 & -3287.18559002 & -6.0 \\
\hline IntBR & 1.12653000 & -3288.30249994 & -3287.17596994 & 0.0 \\
\hline IntCR & 1.12972400 & -3288.27898276 & -3287.14925876 & 16.8 \\
\hline TSDR & 1.12942400 & -3288.27146543 & -3287.14204143 & 21.3 \\
\hline IntER & 1.12674800 & -3288.30828861 & -3287.18154061 & -3.5 \\
\hline IntAS & 1.12136300 & -3288.30738491 & -3287.18602191 & -6.3 \\
\hline IntBS & 1.12630300 & -3288.29280773 & -3287.16650473 & 5.9 \\
\hline IntCS & 1.12953000 & -3288.26969323 & -3287.14016323 & 22.5 \\
\hline TSDS & 1.12765300 & -3288.26593790 & -3287.13828490 & 23.6 \\
\hline IntES & 1.12839000 & -3288.31033762 & -3287.18194762 & -3.8 \\
\hline IntFR & 1.14320300 & -3288.77831001 & -3287.63510701 & 2.2 \\
\hline IntGR1 & 1.15447700 & -3289.96015284 & -3288.80567584 & 9.0 \\
\hline IntGR2 & 1.16129500 & -3289.95252097 & -3288.79122597 & 18.1 \\
\hline TSHR1 & 1.14526400 & -3289.93128710 & -3288.78602310 & 21.4 \\
\hline TSHR2 & 1.15652300 & -3289.93194095 & -3288.77541795 & 28.0 \\
\hline TSHR3 & 1.16043300 & -3289.93658718 & -3288.77615418 & 27.5 \\
\hline TSHR4 & 1.15844200 & -3289.94021118 & -3288.78176918 & 24.0 \\
\hline IntIR & 1.15062500 & -3289.96176805 & -3288.81114305 & 5.6 \\
\hline IntJR & 1.14311800 & -3289.51269795 & -3288.36957995 & -7.7 \\
\hline $\mathbf{H}_{2}$ & -0.0016680 & -1.1797478832 & -1.1814158832 & - \\
\hline$\left[\mathrm{HNEt}_{3}\right]^{+}$ & 0.1815470 & -293.0486141100 & -292.8670671100 & - \\
\hline $\mathbf{N E t}_{3}$ & 0.1663230 & -292.5707203020 & -292.4043973020 & - \\
\hline $\mathbf{R C}$ & 2.180835 & -6155.86499083 & -6153.68415583 & -30.3 \\
\hline
\end{tabular}




\section{$\operatorname{Ir}(\mathrm{III}) / \mathrm{Ir}(\mathrm{V})$ catalytic cycle}

The regeneration of active iridium hydride intermediate through $\operatorname{Ir}(\mathrm{III}) / \mathrm{Ir}(\mathrm{V})$ catalytic cycle was calculated. However, the energy required for the whole reaction process is at least $33.1 \mathrm{kcal} / \mathrm{mol}$, which is too high to complete the catalytic reaction.

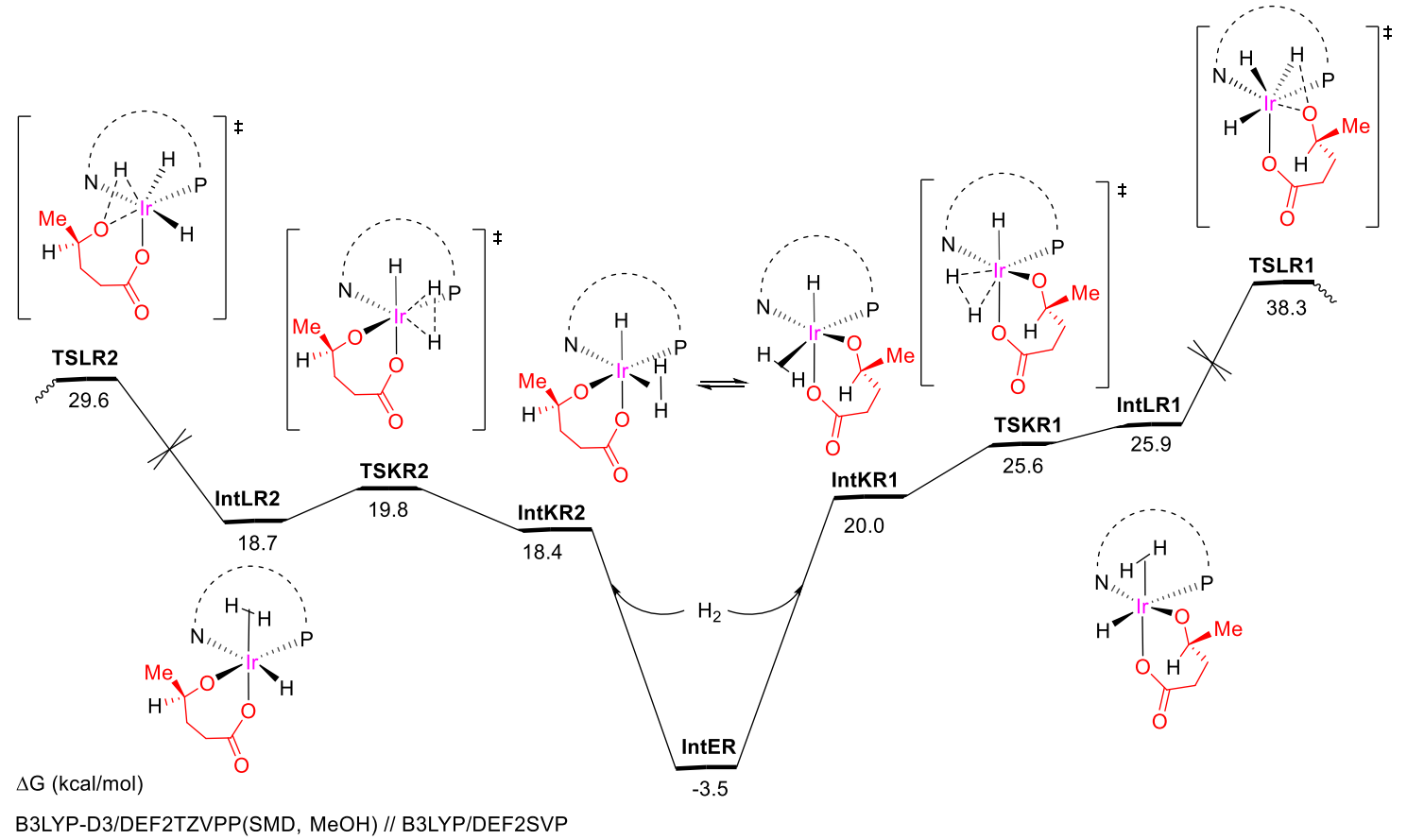

Table S2. Calculated Gibbs energy profile for $\operatorname{Ir}(\mathrm{III}) / \operatorname{Ir}(\mathrm{V})$ catalytic cycle

\begin{tabular}{ccccc}
\hline & $\begin{array}{c}\text { Gibbs energy } \\
\text { corrections }\end{array}$ & $\begin{array}{c}\text { Single-point } \\
\text { energies }\end{array}$ & Gibbs energy & $\Delta$ G \\
\hline IntKR1 & 1.149293 & -3289.47483612 & -3288.325543120 & $\mathbf{2 0 . 0}$ \\
IntKR2 & 1.15091100 & -3289.47889874 & -3288.32798774 & $\mathbf{1 8 . 4}$ \\
TSKR1 & 1.14661900 & -3289.46326719 & -3288.31664819 & $\mathbf{2 5 . 6}$ \\
TSKR2 & 1.14999600 & -3289.47583045 & -3288.32583445 & $\mathbf{1 9 . 8}$ \\
IntLR1 & 1.14861500 & -3289.46470261 & -3288.31608761 & $\mathbf{2 5 . 9}$ \\
IntLR2 & 1.14920500 & -3289.47672686 & -3288.32752186 & $\mathbf{1 8 . 7}$ \\
TSLR1 & 1.14659100 & -3289.44287742 & -3288.29628642 & $\mathbf{3 8 . 3}$ \\
TSLR2 & 1.14787600 & -3289.45803879 & -3288.31016279 & $\mathbf{2 9 . 6}$ \\
\hline
\end{tabular}




\section{Other H-H metathesis process}

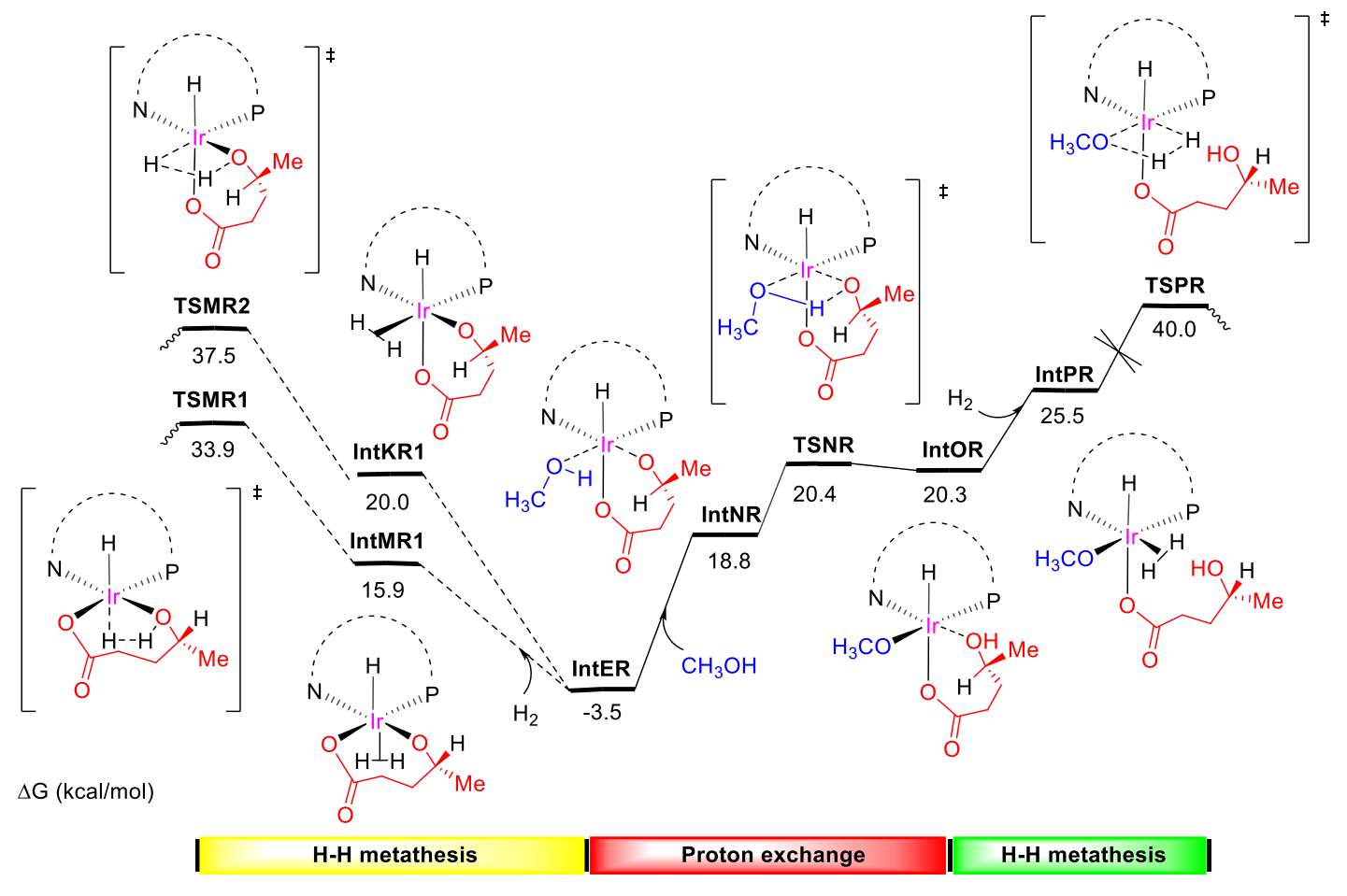

B3LYP-D3/DEF2TZVPP(SMD, MeOH) // B3LYP/DEF2SVP

Table S3. Calculated Gibbs energy profile for the $\mathrm{H}-\mathrm{H}$ metathesis process

\begin{tabular}{ccccc}
\hline & $\begin{array}{c}\text { Gibbs energy } \\
\text { corrections }\end{array}$ & $\begin{array}{c}\text { Single-point } \\
\text { energies }\end{array}$ & Gibbs energy & $\Delta \mathbf{G}$ \\
\hline IntMR1 & 1.14495700 & -3289.47694962 & -3288.33199262 & $\mathbf{1 5 . 9}$ \\
TSMR1 & 1.14430300 & -3289.44759356 & -3288.30329056 & $\mathbf{3 3 . 9}$ \\
TSMR2 & 1.14935500 & -3289.44690311 & -3288.29754811 & $\mathbf{3 7 . 5}$ \\
IntNR & 1.18505200 & -3404.09323995 & -3402.90818795 & $\mathbf{1 8 . 8}$ \\
TSNR & 1.18194800 & -3404.08755350 & -3402.90560550 & $\mathbf{2 0 . 4}$ \\
IntOR & 1.18411800 & -3404.08995985 & -3402.90584185 & $\mathbf{2 0 . 3}$ \\
IntPR & 1.19057600 & -3405.26950560 & -3404.07892960 & $\mathbf{2 5 . 5}$ \\
TSPR & 1.19279700 & -3405.24790874 & -3404.05511174 & $\mathbf{4 0 . 5}$ \\
\hline
\end{tabular}


We calculated the Gibbs energy change of the dissociation process as illustrated in following figure. The generation of IntAR from the resting state (RC) needs a Gibbs energy change of $18.3 \mathrm{kcal} / \mathrm{mol}$.

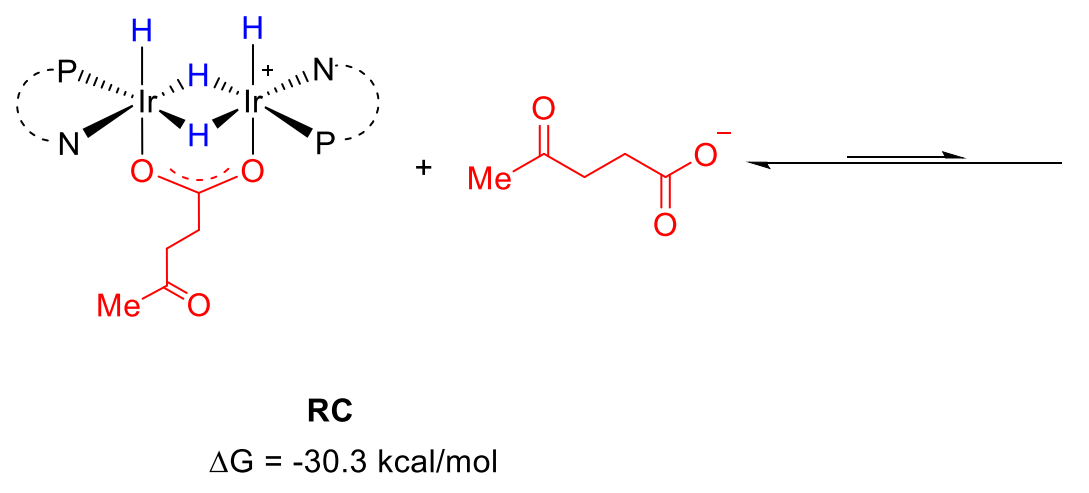

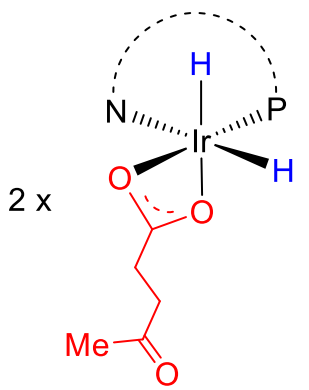

IntAR

$\Delta \mathrm{G}=-12.0 \mathrm{kcal} / \mathrm{mol}$

Figure S4. The Gibbs energy change of the dissociation process. 


\section{The Cartesian coordinates $(\AA)$ for the optimized structures in main text.}

\section{IntAR}

\begin{tabular}{|c|c|c|c|}
\hline $\operatorname{Ir}$ & 0.43920700 & 0.20042200 & 1. 18760600 \\
\hline $\mathrm{P}$ & -1.23575000 & 0.11801200 & -0.31311300 \\
\hline 0 & 2. 12002000 & -3.26233500 & -0.86509800 \\
\hline $\mathrm{N}$ & 1. 77699800 & -1.31540200 & 0.18837600 \\
\hline C & 2. 91252000 & -1.06009900 & -0.72569900 \\
\hline C & 2. 84993000 & -2.28699500 & -1.65479400 \\
\hline C & 1. 46847100 & -2.55631000 & 0.08098100 \\
\hline C & 0.45765300 & -3.24590400 & 0.91343000 \\
\hline $\mathrm{C}$ & 0.73956200 & -3.48240100 & 2. 26866300 \\
\hline C & -0.21116100 & -4.08168300 & 3. 09643500 \\
\hline C & -1.46353200 & -4.43907700 & 2. 58522800 \\
\hline C & -1.75234000 & -4.18762100 & 1. 24307900 \\
\hline $\mathrm{C}$ & -3.00711000 & -4.53572100 & 0.47696800 \\
\hline C & -2.84316000 & -3.76432800 & -0.84802100 \\
\hline C & -1.30575700 & -3.54362700 & -1.03532600 \\
\hline $\mathrm{C}$ & -0.80259500 & -3.58438800 & 0.40231100 \\
\hline C & -0.68961100 & -4.76699800 & -1.79248300 \\
\hline $\mathrm{C}$ & -0.83933400 & -4.43508600 & -3.27624500 \\
\hline C & -0.76442300 & -2.92826300 & -3.28593600 \\
\hline C & -0.51705400 & -2.15455000 & -4.41864000 \\
\hline C & -0.51184100 & -0.76951800 & -4.29218300 \\
\hline C & -0.76217300 & -0.19130400 & -3.04726700 \\
\hline C & -1.01749300 & -0.94289400 & -1.87869800 \\
\hline C & -1.00174700 & -2.36707600 & -2.00635800 \\
\hline C & -2.94389600 & -0.30338100 & 0.27003900 \\
\hline C & -3.98347500 & -0.51506200 & -0.65137000 \\
\hline C & -5.29782400 & -0.75019000 & -0.22207000 \\
\hline C & -5.53435800 & -0.76328000 & 1. 16366500 \\
\hline $\mathrm{C}$ & -4.52292400 & -0.55640100 & 2. 11421800 \\
\hline C & -3.22066000 & -0.32394100 & 1. 64185600 \\
\hline C & -4.86129200 & -0.58790700 & 3. 61920100 \\
\hline C & -3.61645100 & -0.36675100 & 4. 49760400 \\
\hline C & -5.46865800 & -1.96271500 & 3. 98264000 \\
\hline C & -5.88444400 & 0.52667500 & 3.93834100 \\
\hline C & -6.46357400 & -1.00242700 & -1.20229500 \\
\hline C & -6.01005800 & -0.95193800 & -2.67270100 \\
\hline C & -7.06559900 & -2.40144100 & -0.93329600 \\
\hline C & -7.55493200 & 0.07341300 & -0.99683800 \\
\hline C & -1.47815900 & 1. 85573100 & -0.97188800 \\
\hline C & -0.32323700 & 2. 61034200 & -1.25942300 \\
\hline C & -0.40999300 & 3. 93951300 & -1.68525400 \\
\hline $\mathrm{C}$ & -1.69240600 & 4. 51451800 & -1.79077600 \\
\hline
\end{tabular}




\begin{tabular}{|c|c|c|c|}
\hline $\mathrm{C}$ & -2.86089800 & 3. 80625800 & -1.49715500 \\
\hline $\mathrm{C}$ & -2.73063900 & 2. 46284600 & -1.09238900 \\
\hline $\mathrm{C}$ & -4.26582100 & 4. 43714800 & -1.59457300 \\
\hline $\mathrm{C}$ & -4.96544100 & 4. 35059900 & -0.21824600 \\
\hline $\mathrm{C}$ & -4.21623600 & 5. 91773100 & -2.01327500 \\
\hline $\mathrm{C}$ & -5.10147400 & 3. 66942800 & -2.64477400 \\
\hline $\mathrm{C}$ & 0.83802500 & 4. 79538500 & -1.98689900 \\
\hline $\mathrm{C}$ & 2. 12724200 & 3. 95376000 & -2.00944900 \\
\hline $\mathrm{C}$ & 0.97785900 & 5. 87006400 & -0.88275400 \\
\hline $\mathrm{C}$ & 0.69067000 & 5. 48733200 & -3.36115700 \\
\hline $\mathrm{H}$ & -0.37305000 & -0.87612600 & 1.95510100 \\
\hline $\mathrm{H}$ & 2. 73284700 & -0.12395000 & -1.26958700 \\
\hline $\mathrm{H}$ & 2. 26699200 & -2.09688200 & -2.56985400 \\
\hline $\mathrm{H}$ & 3. 82530900 & -2.71404700 & -1.91803400 \\
\hline $\mathrm{H}$ & 1. 70503300 & -3.17450800 & 2. 67475500 \\
\hline $\mathrm{H}$ & 0.02412500 & -4.26020900 & 4. 14836100 \\
\hline $\mathrm{H}$ & -2.20855800 & -4.91143400 & 3. 23175200 \\
\hline $\mathrm{H}$ & -3.05893700 & -5.62759200 & 0.31388700 \\
\hline $\mathrm{H}$ & -3.92629700 & -4.25422100 & 1. 01504400 \\
\hline $\mathrm{H}$ & -3.34083200 & -2.79337400 & -0.76423600 \\
\hline $\mathrm{H}$ & -3.28506300 & -4.28570900 & -1.71090700 \\
\hline $\mathrm{H}$ & 0.37711900 & -4.84180200 & -1.54057400 \\
\hline $\mathrm{H}$ & -1.17253900 & -5.70948500 & -1.49314800 \\
\hline $\mathrm{H}$ & -0.06678500 & -4.90351800 & -3.90680600 \\
\hline $\mathrm{H}$ & -1.81578400 & -4.76694000 & -3.67443400 \\
\hline $\mathrm{H}$ & -0.33359400 & -2.63474200 & -5.38389400 \\
\hline $\mathrm{H}$ & -0.31983000 & -0.12816700 & -5.15617300 \\
\hline $\mathrm{H}$ & -0.76090400 & 0.89488100 & -2.98876700 \\
\hline $\mathrm{H}$ & -3.75280400 & -0.49738500 & -1.71610300 \\
\hline $\mathrm{H}$ & -6.55180700 & -0.94520900 & 1.51588600 \\
\hline $\mathrm{H}$ & -2.39884600 & -0.15182700 & 2. 33351400 \\
\hline $\mathrm{H}$ & -3.14705300 & 0.61109200 & 4. 31073300 \\
\hline $\mathrm{H}$ & -2.85366800 & -1.14330300 & 4. 33389800 \\
\hline $\mathrm{H}$ & -3.90252900 & -0.39908800 & 5. 56087900 \\
\hline $\mathrm{H}$ & -6.39721500 & -2.16700700 & 3. 42716500 \\
\hline $\mathrm{H}$ & -5.70988900 & -2.00389500 & 5. 05761300 \\
\hline $\mathrm{H}$ & -4.75885900 & -2.77634000 & 3. 76307000 \\
\hline $\mathrm{H}$ & -5.47246900 & 1. 51954800 & 3. 69785800 \\
\hline $\mathrm{H}$ & -6.14259200 & 0.51668200 & 5. 01018100 \\
\hline $\mathrm{H}$ & -6.81948500 & 0.40435300 & 3. 36995200 \\
\hline $\mathrm{H}$ & -5.59588800 & 0.03154800 & -2.94381600 \\
\hline $\mathrm{H}$ & -6.87042900 & -1.14031300 & -3.33402000 \\
\hline $\mathrm{H}$ & -5.24838000 & -1.71497700 & -2.89595000 \\
\hline $\mathrm{H}$ & -6.31010700 & -3.19053400 & -1.07618700 \\
\hline $\mathrm{H}$ & -7.90043700 & -2.60117800 & -1.62512800 \\
\hline
\end{tabular}




\begin{tabular}{|c|c|c|c|}
\hline H & -7.45473700 & -2.49210700 & 0.09221900 \\
\hline $\mathrm{H}$ & -7.15719200 & 1. 08197400 & -1.19199900 \\
\hline $\mathrm{H}$ & -7.95527300 & 0.06424700 & 0.02829600 \\
\hline $\mathrm{H}$ & -8.39882100 & -0.09771100 & -1.68543100 \\
\hline H & 0.65288100 & 2. 15154800 & -1.11644600 \\
\hline $\mathrm{H}$ & -1.76818800 & 5.55530000 & -2.10208200 \\
\hline $\mathrm{H}$ & -3.62722300 & 1. 89517600 & -0.85039700 \\
\hline $\mathrm{H}$ & -5.08189800 & 3. 31008100 & 0.12028900 \\
\hline $\mathrm{H}$ & -5.97108300 & 4. 80051600 & -0.26815600 \\
\hline H & -4.38885300 & 4. 88892600 & 0.55068600 \\
\hline $\mathrm{H}$ & -5.23904200 & 6.32313800 & -2.06739100 \\
\hline H & -3.75622000 & 6.05087700 & -3.00491300 \\
\hline H & -3.65582400 & 6.53103400 & -1.29043200 \\
\hline $\mathrm{H}$ & -5.20178600 & 2. 60425400 & -2.38668800 \\
\hline H & -4.63577700 & 3. 72983000 & -3.64147100 \\
\hline $\mathrm{H}$ & -6.11683900 & 4. 09351200 & -2.71696600 \\
\hline H & 2. 08576800 & 3. 17475000 & -2.78835200 \\
\hline $\mathrm{H}$ & 2. 31839100 & 3. 46316100 & -1.04403900 \\
\hline $\mathrm{H}$ & 2. 98725800 & 4. 60366900 & -2.23891000 \\
\hline $\mathrm{H}$ & 0.09189600 & 6.52258200 & -0.83455300 \\
\hline $\mathrm{H}$ & 1. 85658900 & 6.50851100 & -1.07419100 \\
\hline $\mathrm{H}$ & 1. 10486200 & 5. 39892900 & 0.10427000 \\
\hline $\mathrm{H}$ & -0.17162000 & 6. 17032400 & -3.39920900 \\
\hline $\mathrm{H}$ & 0.56841800 & 4. 74612000 & -4.16744500 \\
\hline $\mathrm{H}$ & 1. 59032700 & 6.08414700 & -3.58404400 \\
\hline $\mathrm{C}$ & 4. 22971500 & -0.93768000 & 0.07401500 \\
\hline $\mathrm{H}$ & 4. 44740300 & -1.90660900 & 0.54579000 \\
\hline $\mathrm{H}$ & 4. 04800600 & -0.23850900 & 0.89959400 \\
\hline $\mathrm{C}$ & 5. 38891300 & -0.43611000 & -0.76466400 \\
\hline $\mathrm{C}$ & 6. 42889300 & -1.29648300 & -1.25936400 \\
\hline $\mathrm{C}$ & 5. 42219000 & 0.91358200 & -1.08536800 \\
\hline $\mathrm{C}$ & 6. 49898000 & -2.69315100 & -0.97262700 \\
\hline $\mathrm{C}$ & 7. 46648200 & -0.73164200 & -2.08452900 \\
\hline $\mathrm{C}$ & 6. 44330800 & 1. 46374800 & -1.89398800 \\
\hline $\mathrm{H}$ & 4. 63321900 & 1. 56544700 & -0.69892200 \\
\hline $\mathrm{C}$ & 7. 51428000 & -3.48331600 & -1.47137500 \\
\hline $\mathrm{H}$ & 5. 73995300 & -3.15276100 & -0.33835800 \\
\hline $\mathrm{C}$ & 8.49893900 & -1.57661000 & -2.58256500 \\
\hline $\mathrm{C}$ & 7. 44490700 & 0.65704500 & -2.38711100 \\
\hline $\mathrm{H}$ & 6. 43138800 & 2.53257800 & -2.12177900 \\
\hline $\mathrm{C}$ & 8.52625700 & -2.92221600 & -2.28816900 \\
\hline $\mathrm{H}$ & 7. 54040800 & -4.54912700 & -1.23035600 \\
\hline $\mathrm{H}$ & 9.27830900 & -1.13209200 & -3.20792300 \\
\hline $\mathrm{H}$ & 8. 23888800 & 1. 07204000 & -3.01398100 \\
\hline$U$ & 9. 32638200 & -3.55648400 & -2.67799600 \\
\hline
\end{tabular}




$\begin{array}{lrrr}\mathrm{C} & 5.64042405 & 4.04573339 & 5.43181200 \\ \mathrm{H} & 4.85398736 & 4.63790338 & 5.92688280 \\ \mathrm{H} & 6.26540624 & 4.74355322 & 4.84848479 \\ \mathrm{H} & 6.27377834 & 3.58715864 & 6.20795139 \\ \mathrm{C} & 5.04109621 & 2.96543369 & 4.53223078 \\ \mathrm{O} & 6.10500638 & 2.37883919 & 3.96240831 \\ \mathrm{C} & 3.86111000 & 2.29519400 & 4.02143600 \\ \mathrm{H} & 3.89361800 & 1.23658400 & 4.32511900 \\ \mathrm{H} & 2.96702000 & 2.72849000 & 4.49923900 \\ \mathrm{C} & 3.73495700 & 2.37747600 & 2.50087600 \\ \mathrm{H} & 3.63727500 & 3.42033500 & 2.15628300 \\ \mathrm{H} & 4.66298400 & 1.99630900 & 2.04096300 \\ \mathrm{C} & 2.57634600 & 1.59495200 & 1.91521200 \\ 0 & 2.03517200 & 0.66812800 & 2.59653100 \\ 0 & 2.16519200 & 1.83814400 & 0.74676300 \\ \mathrm{H} & -0.46149500 & 1.28212800 & 1.91680900\end{array}$

\section{IntBR}

Ir

$\mathrm{P}$

0

$\mathrm{N}$

C

C

C

C

C

C

C

C

C

C

C

C

C

C

C

C

C

C

C

C

C

C
0. 43290700

$-1.07212000$

2. 74591900

1. 99394300

3. 19559400

3. 45070400

1. 88008500

0. 87526900

1. 09397700

0. 13827200

$-1.05463700$

$-1.27621100$

$-2.44453700$

$-2.25055800$

$-0.72474600$

$-0.32339900$

0. 05096200

$-0.03340600$

$-0.11824100$

0. 07682800

$-0.10484700$

$-0.46655000$

$-0.65059000$

$-0.46878000$

$-2.78212200$

$-3.71900900$
$-0.07131200$

0. 17713800

$-2.81149400$

$-1.16343500$

$-0.59627100$

$-1.62461100$

$-2.38307300$

$-3.33249900$

$-3.91872100$

$-4.76435400$

$-5.02910600$

$-4.44171800$

$-4.65559500$

$-3.56652400$

$-3.21652900$

$-3.58369000$

$-4.18566600$

$-3.54450600$

$-2.07373700$

$-1.05412400$

0. 26193000

0. 52910900

$-0.47500500$

$-1.83079200$

$-0.48540600$

$-0.41106400$
1. 33521800

$-0.29992300$

$-1.15808600$

0. 15751900

$-0.50066300$

$-1.61818000$

$-0.22136500$

0. 31076700

1. 56673400

2. 13239000

1. 45180300

0. 20517800

$-0.72778600$

$-1.80071500$

$-1.79364500$

$-0.36872100$

$-2.74716500$

-4. 12954800

$-3.80702500$

$-4.73742800$

$-4.33107100$

$-3.00999200$

$-2.03529000$

$-2.45536100$

$-0.02924900$

$-1.07439700$ 


\begin{tabular}{|c|c|c|c|}
\hline $\mathrm{C}$ & -5.03697800 & -0.85441300 & -0.89944200 \\
\hline $\mathrm{C}$ & -5.38461600 & -1.37018700 & 0.36196300 \\
\hline $\mathrm{C}$ & -4.47987500 & -1.45230800 & 1. 43104000 \\
\hline $\mathrm{C}$ & -3.16678200 & -1.00116300 & 1. 21233800 \\
\hline $\mathrm{C}$ & -4.94314700 & -2.01907500 & 2. 78918300 \\
\hline $\mathrm{C}$ & -3.80571200 & -2.03705600 & 3. 82643400 \\
\hline $\mathrm{C}$ & -5.44838300 & -3.46824200 & 2. 60073300 \\
\hline $\mathrm{C}$ & -6.09190200 & -1.14481400 & 3. 34402700 \\
\hline $\mathrm{C}$ & -6.08702600 & -0.81604100 & -2.03014800 \\
\hline $\mathrm{C}$ & -5.54248300 & -0.14959700 & -3.30660000 \\
\hline $\mathrm{C}$ & -6.50762800 & -2.26240200 & -2.38121600 \\
\hline $\mathrm{C}$ & -7.32912800 & -0.02083200 & -1.56577300 \\
\hline $\mathrm{C}$ & -1.39379600 & 2. 00566500 & -0.51741900 \\
\hline $\mathrm{C}$ & -0.31931400 & 2. 85983900 & -0.84284400 \\
\hline $\mathrm{C}$ & -0.49417800 & 4. 24419800 & -0.93402100 \\
\hline $\mathrm{C}$ & -1.76768200 & 4. 76763000 & -0.63114600 \\
\hline $\mathrm{C}$ & -2.84882900 & 3.95885500 & -0.27056700 \\
\hline $\mathrm{C}$ & -2.64285200 & 2.56553400 & -0.23269600 \\
\hline $\mathrm{C}$ & -4.24081200 & 4. 53034600 & 0.07380500 \\
\hline $\mathrm{C}$ & -4.63249400 & 4. 10699600 & 1. 50866900 \\
\hline $\mathrm{C}$ & -4.27625200 & 6.06761400 & 0.00237300 \\
\hline $\mathrm{C}$ & -5.28321700 & 3.97857000 & -0.92595600 \\
\hline $\mathrm{C}$ & 0.64855200 & 5. 20193800 & -1.33640100 \\
\hline $\mathrm{C}$ & 1. 90409400 & 4. 44589400 & -1.80979600 \\
\hline $\mathrm{C}$ & 1. 04234500 & 6.07237500 & -0.11942500 \\
\hline $\mathrm{C}$ & 0.17278300 & 6.11572100 & -2.49005200 \\
\hline $\mathrm{H}$ & -0.34668200 & -1.36901500 & 1. 76003900 \\
\hline $\mathrm{H}$ & -0.64905800 & 0.70398500 & 2. 20174300 \\
\hline $\mathrm{H}$ & 2. 95108700 & 0.39795900 & -0.89321900 \\
\hline $\mathrm{H}$ & 2. 99782300 & -1.32361900 & -2.57602400 \\
\hline $\mathrm{H}$ & 4. 50341000 & -1.88720800 & -1.77479100 \\
\hline $\mathrm{H}$ & 2. 01229400 & -3.68721300 & 2. 11004200 \\
\hline $\mathrm{H}$ & 0.32290200 & -5.21233400 & 3. 11184800 \\
\hline $\mathrm{H}$ & -1.80283100 & -5.69666900 & 1. 88855200 \\
\hline $\mathrm{H}$ & -2.39866600 & -5.67168000 & -1.16010000 \\
\hline $\mathrm{H}$ & -3.41842300 & -4.57460100 & -0.21937500 \\
\hline $\mathrm{H}$ & -2.82638400 & -2.67687200 & -1.52425600 \\
\hline $\mathrm{H}$ & -2.59070400 & -3.87725600 & -2.80039500 \\
\hline $\mathrm{H}$ & 1. 10196700 & -4.22969400 & -2.42738400 \\
\hline $\mathrm{H}$ & -0.35466300 & -5.20755800 & -2.70089000 \\
\hline $\mathrm{H}$ & 0.82494000 & -3.78924800 & -4.77561100 \\
\hline $\mathrm{H}$ & -0.94005300 & -3.86299900 & -4.67589100 \\
\hline $\mathrm{H}$ & 0.35417600 & -1.29544500 & -5.76737100 \\
\hline $\mathrm{H}$ & 0.02719900 & 1. 09036400 & -5.03154500 \\
\hline $\mathrm{H}$ & -0.61601900 & 1.56967700 & -2.73627100 \\
\hline
\end{tabular}




\begin{tabular}{|c|c|c|c|}
\hline H & -3.40449400 & -0.00201900 & -2.03388900 \\
\hline $\mathrm{H}$ & -6.40686500 & -1.72161300 & 0.51614100 \\
\hline $\mathrm{H}$ & -2.41995100 & -1.04422800 & 2. 00179900 \\
\hline $\mathrm{H}$ & -3.42152500 & -1.02631600 & 4. 03262000 \\
\hline $\mathrm{H}$ & -2.95886500 & -2.65859400 & 3. 49753400 \\
\hline $\mathrm{H}$ & -4.17622700 & -2.45191700 & 4. 77732500 \\
\hline H & -6.29481300 & -3.52371900 & 1. 89879400 \\
\hline $\mathrm{H}$ & -5.78789700 & -3.88572900 & 3. 56299800 \\
\hline $\mathrm{H}$ & -4.64671000 & -4.11757600 & 2. 21392100 \\
\hline H & -5.76080600 & -0.10408800 & 3. 48801700 \\
\hline $\mathrm{H}$ & -6.43354400 & -1.53089600 & 4. 31866300 \\
\hline H & -6.96183800 & -1.12940900 & 2. 66946700 \\
\hline $\mathrm{H}$ & -5.23218600 & 0.89126400 & -3.12600700 \\
\hline H & -6.32568900 & -0.13288800 & -4.08077400 \\
\hline H & -4.68124200 & -0.69528800 & -3.72189200 \\
\hline $\mathrm{H}$ & -5.64450700 & -2.84949100 & -2.73354200 \\
\hline H & -7.26647900 & -2.25974200 & -3.18112200 \\
\hline $\mathrm{H}$ & -6.93815900 & -2.78651400 & -1.51412600 \\
\hline $\mathrm{H}$ & -7.06195700 & 1. 01607300 & -1.30753900 \\
\hline $\mathrm{H}$ & -7.80653700 & -0.47352500 & -0.68343000 \\
\hline $\mathrm{H}$ & -8.08406900 & 0.01340200 & -2.36831300 \\
\hline $\mathrm{H}$ & 0.66846200 & 2. 43155500 & -0.99216500 \\
\hline $\mathrm{H}$ & -1.90549100 & 5.84685300 & -0.67779000 \\
\hline $\mathrm{H}$ & -3.47288000 & 1. 91552900 & 0.03908000 \\
\hline $\mathrm{H}$ & -4.67273400 & 3. 01275800 & 1. 61873000 \\
\hline $\mathrm{H}$ & -5.62690100 & 4. 50591700 & 1. 76950400 \\
\hline $\mathrm{H}$ & -3.90761100 & 4. 49039700 & 2. 24427300 \\
\hline $\mathrm{H}$ & -5.28431200 & 6.42959200 & 0.25990200 \\
\hline $\mathrm{H}$ & -4.04128900 & 6.43889000 & -1.00736100 \\
\hline $\mathrm{H}$ & -3.56875400 & 6.52817300 & 0.70939100 \\
\hline $\mathrm{H}$ & -5.33339000 & 2. 87950100 & -0.89715500 \\
\hline $\mathrm{H}$ & -5.03565900 & 4. 27666700 & -1.95740300 \\
\hline $\mathrm{H}$ & -6.28877400 & 4. 36591800 & -0.69148400 \\
\hline $\mathrm{H}$ & 1. 68495700 & 3. 79079500 & -2.66896100 \\
\hline $\mathrm{H}$ & 2. 34975400 & 3. 84455500 & -1.00410700 \\
\hline $\mathrm{H}$ & 2. 66668900 & 5. 17200400 & -2.13456200 \\
\hline $\mathrm{H}$ & 0.17816100 & 6.61975200 & 0.28954400 \\
\hline $\mathrm{H}$ & 1. 80183200 & 6.81580900 & -0.41428900 \\
\hline $\mathrm{H}$ & 1. 47860100 & 5. 45162400 & 0.67736200 \\
\hline $\mathrm{H}$ & -0.67644400 & 6. 75368900 & -2.20028100 \\
\hline $\mathrm{H}$ & -0.13618000 & 5. 52291800 & -3.36649000 \\
\hline $\mathrm{H}$ & 0.99110200 & 6. 78369200 & -2.80478200 \\
\hline $\mathrm{C}$ & 4. 33096700 & -0.43975100 & 0.53724200 \\
\hline $\mathrm{H}$ & 4. 54189200 & -1.42175400 & 0.98732900 \\
\hline . & 3.92115700 & 0.19354100 & 1. 33399900 \\
\hline
\end{tabular}




\begin{tabular}{lrrr} 
C & 5.58650900 & 0.21126600 & -0.00916500 \\
$\mathrm{C}$ & 6.73044700 & -0.55276700 & -0.43083900 \\
$\mathrm{C}$ & 5.62054300 & 1.59563300 & -0.10990300 \\
$\mathrm{C}$ & 6.80690200 & -1.97555300 & -0.33698600 \\
$\mathrm{C}$ & 7.87459000 & 0.14095700 & -0.96505000 \\
$\mathrm{C}$ & 6.74809300 & 2.26814500 & -0.63864200 \\
$\mathrm{H}$ & 4.76713600 & 2.18726600 & 0.24206500 \\
$\mathrm{C}$ & 7.92687500 & -2.66978200 & -0.74787800 \\
$\mathrm{H}$ & 5.96495700 & -2.53514700 & 0.07301200 \\
$\mathrm{C}$ & 9.01271500 & -0.60678300 & -1.38146000 \\
$\mathrm{C}$ & 7.85019000 & 1.55900400 & -1.06214600 \\
$\mathrm{H}$ & 6.73489400 & 3.35927300 & -0.70093400 \\
$\mathrm{C}$ & 9.04415800 & -1.98061700 & -1.27922500 \\
$\mathrm{H}$ & 7.95270000 & -3.75910900 & -0.65977400 \\
$\mathrm{H}$ & 9.87147000 & -0.06345900 & -1.78598700 \\
$\mathrm{H}$ & 8.72428700 & 2.07387100 & -1.47073500 \\
$\mathrm{H}$ & 9.92650900 & -2.53881800 & -1.60246900 \\
$\mathrm{C}$ & 2.98355800 & -0.40233100 & 5.11319000 \\
$\mathrm{H}$ & 3.94833000 & 0.06330300 & 5.36584100 \\
$\mathrm{H}$ & 3.12553200 & -1.45529100 & 4.83842300 \\
$\mathrm{H}$ & 2.35329000 & -0.35417700 & 6.01819400 \\
$\mathrm{C}$ & 2.28786500 & 0.34059800 & 3.99509200 \\
$\mathrm{O}$ & 1.74287600 & -0.31487200 & 3.11272100 \\
$\mathrm{C}$ & 2.31652300 & 1.84940700 & 4.08486000 \\
$\mathrm{H}$ & 1.99913600 & 2.10027900 & 5.11471700 \\
$\mathrm{H}$ & 3.38534000 & 2.13717000 & 4.05000600 \\
$\mathrm{C}$ & 1.53682000 & 2.66627900 & 3.05205600 \\
$\mathrm{H}$ & 0.48690100 & 2.34516100 & 3.01792400 \\
$\mathrm{H}$ & 1.57825000 & 3.71921900 & 3.36563200 \\
$\mathrm{C}$ & 2.18402600 & 2.57066200 & 1.65659400 \\
O & 3.09287500 & 3.36253000 & 1.38133500 \\
$\mathrm{O}$ & 1.77137200 & 1.64103000 & 0.87478200 \\
& & & \\
\hline
\end{tabular}

\section{IntCR}

$\begin{array}{rrrr}\text { Ir } & -0.65180500 & -0.18164600 & -1.55144200 \\ \text { P } & 0.91939000 & 0.13441400 & 0.42544900 \\ 0 & -2.77137500 & -2.84793700 & 0.92393300 \\ \mathrm{~N} & -1.97728000 & -1.22334000 & -0.37563500 \\ \mathrm{C} & -3.23062700 & -0.65190900 & 0.19826700 \\ \mathrm{C} & -3.53017800 & -1.66627100 & 1.31332100 \\ \mathrm{C} & -1.85971000 & -2.44045800 & 0.03753000 \\ \mathrm{C} & -0.81224200 & -3.38969100 & -0.39688500 \\ \mathrm{C} & -0.93354100 & -4.00784600 & -1.65168600 \\ \mathrm{C} & 0.06882500 & -4.85639900 & -2.12177200\end{array}$




\begin{tabular}{|c|c|c|c|}
\hline $\mathrm{C}$ & 1. 21166100 & -5.08714000 & -1.34803000 \\
\hline $\mathrm{C}$ & 1. 33180300 & -4.47406200 & -0.10035800 \\
\hline $\mathrm{C}$ & 2. 42812200 & -4.65804600 & 0.92253300 \\
\hline $\mathrm{C}$ & 2. 12582200 & -3.57070500 & 1. 97164700 \\
\hline $\mathrm{C}$ & 0.59777300 & -3.25702700 & 1. 84262300 \\
\hline $\mathrm{C}$ & 0.32645300 & -3.61943200 & 0.38547100 \\
\hline $\mathrm{C}$ & -0.22891200 & -4.26095200 & 2. 71444100 \\
\hline $\mathrm{C}$ & -0.25572500 & -3.65129500 & 4. 11373700 \\
\hline $\mathrm{C}$ & -0.16585700 & -2.17222200 & 3. 83328600 \\
\hline $\mathrm{C}$ & -0.41653700 & -1.17679300 & 4. 77591400 \\
\hline $\mathrm{C}$ & -0.20901300 & 0.14949400 & 4. 41544900 \\
\hline $\mathrm{C}$ & 0.21900300 & 0.45208400 & 3. 12268700 \\
\hline $\mathrm{C}$ & 0.44953100 & -0.52705500 & 2. 13248100 \\
\hline $\mathrm{C}$ & 0.26106400 & -1.89327200 & 2.51057100 \\
\hline $\mathrm{C}$ & 2. 64914000 & -0.47923800 & 0.20167900 \\
\hline $\mathrm{C}$ & 3.57286800 & -0.38380700 & 1. 25662900 \\
\hline $\mathrm{C}$ & 4. 89918300 & -0.80620500 & 1. 09548700 \\
\hline $\mathrm{C}$ & 5. 26734400 & -1.31759700 & -0.16232400 \\
\hline C & 4. 37649100 & -1.41639000 & -1.24141400 \\
\hline $\mathrm{C}$ & 3. 05230500 & -0.99183400 & -1.03589600 \\
\hline $\mathrm{C}$ & 4. 86893200 & -1.96440100 & -2.59672800 \\
\hline $\mathrm{C}$ & 3. 74424200 & -1.99938500 & -3.64703100 \\
\hline $\mathrm{C}$ & 5. 40619900 & -3.40237500 & -2.41228800 \\
\hline $\mathrm{C}$ & 6.00261200 & -1.05886100 & -3.13225700 \\
\hline $\mathrm{C}$ & 5. 93937500 & -0.74499800 & 2. 23389900 \\
\hline $\mathrm{C}$ & 5. 36681800 & -0.10135700 & 3. 50972000 \\
\hline $\mathrm{C}$ & 6. 39900600 & -2.18037200 & 2. 58064500 \\
\hline $\mathrm{C}$ & 7. 16077200 & 0.08833700 & 1. 78121400 \\
\hline $\mathrm{C}$ & 1. 16651600 & 1. 96619100 & 0.62871700 \\
\hline $\mathrm{C}$ & 0.08686300 & 2. 78882100 & 1. 01363100 \\
\hline $\mathrm{C}$ & 0.23443700 & 4. 17562800 & 1. 12301200 \\
\hline $\mathrm{C}$ & 1. 47599300 & 4. 73439400 & 0.75763500 \\
\hline $\mathrm{C}$ & 2. 55305700 & 3. 95896500 & 0.31877600 \\
\hline $\mathrm{C}$ & 2.38201400 & 2.56190700 & 0.27735300 \\
\hline $\mathrm{C}$ & 3. 90463200 & 4.57040300 & -0.10648200 \\
\hline $\mathrm{C}$ & 4. 20240000 & 4. 18321900 & -1.57381300 \\
\hline $\mathrm{C}$ & 3. 90887400 & 6. 10631200 & -0.00539700 \\
\hline $\mathrm{C}$ & 5.02708200 & 4. 02609000 & 0.80714300 \\
\hline $\mathrm{C}$ & -0.89091800 & 5. 09700100 & 1. 64163400 \\
\hline $\mathrm{C}$ & -2.14296200 & 4. 30594100 & 2. 06283100 \\
\hline $\mathrm{C}$ & -1.30557400 & 6.09362300 & 0.53358800 \\
\hline $\mathrm{C}$ & -0.37109800 & 5. 87656400 & 2. 87285400 \\
\hline $\mathrm{H}$ & 0.12649100 & -1.48088700 & -1.96649700 \\
\hline $\mathrm{H}$ & -3.00884500 & 0.34796500 & 0.58607500 \\
\hline $\mathrm{H}$ & -3.14638000 & -1.34514700 & 2. 29390100 \\
\hline
\end{tabular}




\begin{tabular}{|c|c|c|c|}
\hline H & -4.58518000 & -1.95021600 & 1. 40182100 \\
\hline $\mathrm{H}$ & -1.81083200 & -3.80068000 & -2.26774400 \\
\hline $\mathrm{H}$ & -0.03858100 & -5.33199600 & -3.09952100 \\
\hline $\mathrm{H}$ & 1.99825400 & -5.75367300 & -1.71277800 \\
\hline H & 2. 37296800 & -5.67377100 & 1. 35424500 \\
\hline $\mathrm{H}$ & 3. 43558000 & -4.55493700 & 0.48922300 \\
\hline H & 2. 69998300 & -2.66708600 & 1. 73800200 \\
\hline $\mathrm{H}$ & 2. 39157900 & -3.87104300 & 2. 99640500 \\
\hline $\mathrm{H}$ & -1.25263200 & -4.31358400 & 2. 31689100 \\
\hline H & 0.19609000 & -5.27505700 & 2. 67512500 \\
\hline $\mathrm{H}$ & -1.15681200 & -3.92099600 & 4. 68755000 \\
\hline H & 0.61128500 & -3.97332200 & 4. 71883000 \\
\hline $\mathrm{H}$ & -0.75121600 & -1.44378000 & 5. 78212000 \\
\hline H & -0.37306000 & 0.95873200 & 5. 13136900 \\
\hline H & 0.38582400 & 1. 49858200 & 2. 88326300 \\
\hline $\mathrm{H}$ & 3. 23939600 & 0.02448900 & 2. 21006300 \\
\hline $\mathrm{H}$ & 6. 29775000 & -1.64865100 & -0.30668300 \\
\hline $\mathrm{H}$ & 2. 31837100 & -1.03731000 & -1.83673500 \\
\hline $\mathrm{H}$ & 3. 33262400 & -0.99705100 & -3.83879900 \\
\hline $\mathrm{H}$ & 2.91395100 & -2.65238900 & -3.33665300 \\
\hline $\mathrm{H}$ & 4. 13680300 & -2.38794500 & -4.60012100 \\
\hline $\mathrm{H}$ & 6.24796200 & -3.44243400 & -1.70367900 \\
\hline $\mathrm{H}$ & 5.76340900 & -3.80590000 & -3.37407000 \\
\hline $\mathrm{H}$ & 4. 61676400 & -4.07256900 & -2.03594800 \\
\hline $\mathrm{H}$ & 5. 64723600 & -0.02606400 & -3.27491200 \\
\hline $\mathrm{H}$ & 6. 36583100 & -1.43117300 & -4.10439800 \\
\hline $\mathrm{H}$ & 6. 86334900 & -1.02581600 & -2.44665700 \\
\hline $\mathrm{H}$ & 5. 03301400 & 0.93297400 & 3. 33371600 \\
\hline $\mathrm{H}$ & 6.14195000 & -0.07131700 & 4. 29155300 \\
\hline $\mathrm{H}$ & 4. 51526600 & -0.67133200 & 3. 91231100 \\
\hline $\mathrm{H}$ & 5. 55113100 & -2.79376800 & 2. 92530800 \\
\hline $\mathrm{H}$ & 7. 15259600 & -2.15988200 & 3. 38517500 \\
\hline $\mathrm{H}$ & 6.84992400 & -2.68818000 & 1. 71426500 \\
\hline $\mathrm{H}$ & 6. 86461700 & 1. 11828300 & 1. 52668000 \\
\hline $\mathrm{H}$ & 7. 65531200 & -0.34601200 & 0.89916000 \\
\hline $\mathrm{H}$ & 7. 91005100 & 0.14003500 & 2. 58809200 \\
\hline $\mathrm{H}$ & -0.87965100 & 2.33705500 & 1. 22031500 \\
\hline $\mathrm{H}$ & 1. 59121500 & 5.81562500 & 0.81952800 \\
\hline $\mathrm{H}$ & 3. 21043200 & 1.93513700 & -0.04862000 \\
\hline $\mathrm{H}$ & 4. 25135800 & 3. 09233100 & -1.70994800 \\
\hline $\mathrm{H}$ & 5. 16872300 & 4. 60603200 & -1.89573300 \\
\hline $\mathrm{H}$ & 3. 42036400 & 4. 56706700 & -2.24797500 \\
\hline $\mathrm{H}$ & 4. 88799700 & 6. 49812200 & -0.32354100 \\
\hline $\mathrm{H}$ & 3. 73526600 & 6.45170000 & 1. 02579700 \\
\hline . & 3. 14375100 & 6.56257300 & -0.65253400 \\
\hline
\end{tabular}




\begin{tabular}{|c|c|c|c|}
\hline H & 5. 10380400 & 2. 92955700 & 0.75223200 \\
\hline $\mathrm{H}$ & 4. 84492000 & 4. 29824900 & 1. 85929500 \\
\hline $\mathrm{H}$ & 6.00350900 & 4. 44495700 & 0.51177100 \\
\hline $\mathrm{H}$ & -1.91917600 & 3. 58107100 & 2. 86292500 \\
\hline $\mathrm{H}$ & -2.58963400 & 3. 77839300 & 1. 20723900 \\
\hline $\mathrm{H}$ & -2.90115600 & 5.00249100 & 2. 45537400 \\
\hline H & -0.45040700 & 6.68640600 & 0.17176900 \\
\hline $\mathrm{H}$ & -2.05702400 & 6.80051300 & 0.92383700 \\
\hline $\mathrm{H}$ & -1.75519400 & 5.55837600 & -0.31615000 \\
\hline H & 0.48804300 & 6.52032500 & 2. 62847100 \\
\hline $\mathrm{H}$ & -0.05783800 & 5. 18947200 & 3. 67606600 \\
\hline $\mathrm{H}$ & -1.16630400 & 6.52610200 & 3. 27429400 \\
\hline $\mathrm{C}$ & -4.31636400 & -0.51374100 & -0.88985400 \\
\hline $\mathrm{H}$ & -4.51134200 & -1.49948200 & -1.33811100 \\
\hline H & -3.88265600 & 0.11610800 & -1.67684200 \\
\hline $\mathrm{C}$ & -5.58536400 & 0.13947600 & -0.37523000 \\
\hline $\mathrm{C}$ & -6.75836300 & -0.61798300 & -0.02872900 \\
\hline $\mathrm{C}$ & -5.59701700 & 1. 51929600 & -0.21865100 \\
\hline $\mathrm{C}$ & -6.85460300 & -2.03549800 & -0.17201100 \\
\hline $\mathrm{C}$ & -7.91143300 & 0.07786000 & 0.48386900 \\
\hline $\mathrm{C}$ & -6.73446400 & 2. 19344600 & 0.28583000 \\
\hline $\mathrm{H}$ & -4.71560300 & 2. 10990600 & -0.49463800 \\
\hline $\mathrm{C}$ & -8.00064300 & -2.72298400 & 0.17298300 \\
\hline $\mathrm{H}$ & -6.00807900 & -2.59662600 & -0.57040700 \\
\hline $\mathrm{C}$ & -9.07733900 & -0.66278900 & 0.82993200 \\
\hline $\mathrm{C}$ & -7.86682600 & 1. 49074900 & 0.63315500 \\
\hline $\mathrm{H}$ & -6.70259100 & 3. 28072400 & 0.39170600 \\
\hline $\mathrm{C}$ & -9.12693700 & -2.03190100 & 0.68225100 \\
\hline $\mathrm{H}$ & -8.04087100 & -3.80821600 & 0.04770000 \\
\hline $\mathrm{H}$ & -9.94250900 & -0.11760500 & 1. 21789500 \\
\hline $\mathrm{H}$ & -8.74854800 & 2. 00704200 & 1. 02308300 \\
\hline $\mathrm{H}$ & -10.03042800 & -2.58459900 & 0.95203600 \\
\hline $\mathrm{C}$ & 0.18386500 & -0.09430800 & -4.62808300 \\
\hline $\mathrm{H}$ & -0.74476700 & -0.37034300 & -5.14730800 \\
\hline $\mathrm{H}$ & 0.69599600 & -1.00260400 & -4.29065100 \\
\hline $\mathrm{H}$ & 0.83481100 & 0.43084200 & -5.35036700 \\
\hline $\mathrm{C}$ & -0.08023200 & 0.85097900 & -3.46781600 \\
\hline 0 & 0.82299500 & 0.97805200 & -2.55941000 \\
\hline $\mathrm{C}$ & -0.98146400 & 2. 03600700 & -3.79845100 \\
\hline $\mathrm{H}$ & -0.50965000 & 2.50638100 & -4.68364100 \\
\hline $\mathrm{H}$ & -1.95728300 & 1. 66376400 & -4.14973100 \\
\hline $\mathrm{C}$ & -1.14449200 & 3. 09880700 & -2.71154200 \\
\hline $\mathrm{H}$ & -0.15342300 & 3. 33180100 & -2.28770900 \\
\hline $\mathrm{H}$ & -1.54219200 & 4. 02145000 & -3.15628900 \\
\hline $\mathrm{C}$ & -2.06852900 & 2. 71675000 & -1.54890100 \\
\hline
\end{tabular}




$\begin{array}{lrrr}0 & -2.92534300 & 3.52303800 & -1.17163300 \\ 0 & -1.88448800 & 1.56977700 & -0.99440500 \\ \mathrm{H} & -1.68558700 & -0.55720900 & -2.71301800\end{array}$

\section{TSDR}

\begin{tabular}{|c|c|c|c|}
\hline Ir & -0.56996800 & -0.12886700 & -1.41234400 \\
\hline $\mathrm{P}$ & 0.92396900 & 0.10903900 & 0.44293800 \\
\hline 0 & -2.84463800 & -2.81495500 & 0.94013700 \\
\hline $\mathrm{N}$ & -1.95494200 & -1.16532800 & -0.27214000 \\
\hline $\mathrm{C}$ & -3.21360600 & -0.58695000 & 0.28363300 \\
\hline $\mathrm{C}$ & -3.55115400 & -1.61692100 & 1. 37085200 \\
\hline $\mathrm{C}$ & -1.89399000 & -2.40087500 & 0.09141400 \\
\hline $\mathrm{C}$ & -0.87825400 & -3.37014100 & -0.37347200 \\
\hline $\mathrm{C}$ & -1.03633800 & -3.96865400 & -1.63359900 \\
\hline $\mathrm{C}$ & -0.06053200 & -4.83021200 & -2.13616900 \\
\hline $\mathrm{C}$ & 1. 09162500 & -5.09719700 & -1.38851700 \\
\hline $\mathrm{C}$ & 1. 24679600 & -4.50668700 & -0.13380200 \\
\hline $\mathrm{C}$ & 2. 35690700 & -4.72788400 & 0.86644700 \\
\hline $\mathrm{C}$ & 2. 09788400 & -3.64707000 & 1. 93403200 \\
\hline $\mathrm{C}$ & 0.57495700 & -3.29830800 & 1. 83703300 \\
\hline $\mathrm{C}$ & 0.26949500 & -3.63787700 & 0.38188600 \\
\hline $\mathrm{C}$ & -0.26037600 & -4.28937800 & 2. 71456600 \\
\hline $\mathrm{C}$ & -0.24212000 & -3.69480200 & 4. 12043900 \\
\hline $\mathrm{C}$ & -0.12514400 & -2.21539200 & 3. 85186000 \\
\hline $\mathrm{C}$ & -0.33871100 & -1.22261800 & 4. 80641500 \\
\hline $\mathrm{C}$ & -0.11879400 & 0.10296700 & 4. 45087600 \\
\hline $\mathrm{C}$ & 0.28565600 & 0.40816500 & 3. 15106700 \\
\hline $\mathrm{C}$ & 0.48186400 & -0.56849900 & 2. 15101500 \\
\hline $\mathrm{C}$ & 0.28000700 & -1.93392400 & 2. 52294300 \\
\hline $\mathrm{C}$ & 2. 64133000 & -0.51041300 & 0.15683800 \\
\hline $\mathrm{C}$ & 3.57386100 & -0.50650200 & 1. 20810700 \\
\hline $\mathrm{C}$ & 4. 90240200 & -0.89999000 & 0.99631600 \\
\hline $\mathrm{C}$ & 5. 26264400 & -1.29045400 & -0.30612300 \\
\hline $\mathrm{C}$ & 4. 36126000 & -1.29671400 & -1.38128000 \\
\hline $\mathrm{C}$ & 3. 03750800 & -0.89941000 & -1.12714700 \\
\hline $\mathrm{C}$ & 4. 83939700 & -1.71623000 & -2.78644700 \\
\hline $\mathrm{C}$ & 3. 69292500 & -1.69913000 & -3.81366100 \\
\hline $\mathrm{C}$ & 5. 41833900 & -3.14877100 & -2.73286000 \\
\hline $\mathrm{C}$ & 5. 93564500 & -0.73581700 & -3.26465100 \\
\hline $\mathrm{C}$ & 5.95528700 & -0.92474000 & 2. 12495400 \\
\hline $\mathrm{C}$ & 5.38159600 & -0.43113400 & 3. 46573500 \\
\hline $\mathrm{C}$ & 6.46245700 & -2.37277600 & 2. 31952800 \\
\hline $\mathrm{C}$ & 7. 14513800 & -0.01307900 & 1. 74594800 \\
\hline C & 1. 19601200 & 1. 93637600 & 0.66714500 \\
\hline
\end{tabular}




\begin{tabular}{|c|c|c|c|}
\hline $\mathrm{C}$ & 0.11942500 & 2. 76650100 & 1. 04398900 \\
\hline $\mathrm{C}$ & 0.28087200 & 4. 15001200 & 1. 16818300 \\
\hline $\mathrm{C}$ & 1.53676500 & 4. 69716400 & 0.83452600 \\
\hline $\mathrm{C}$ & 2. 61469300 & 3.91379600 & 0.41297000 \\
\hline $\mathrm{C}$ & 2. 42719400 & 2.51925000 & 0.35098500 \\
\hline $\mathrm{C}$ & 3.98434400 & 4. 51310800 & 0.02982400 \\
\hline $\mathrm{C}$ & 4. 32912600 & 4. 12051400 & -1.42566900 \\
\hline $\mathrm{C}$ & 3. 99765600 & 6.04920500 & 0.12724000 \\
\hline $\mathrm{C}$ & 5.07023800 & 3. 96134200 & 0.98232500 \\
\hline $\mathrm{C}$ & -0.84750800 & 5.08108100 & 1. 66189300 \\
\hline $\mathrm{C}$ & -2.11431700 & 4. 30051000 & 2. 05785800 \\
\hline $\mathrm{C}$ & -1.23074300 & 6. 07689300 & 0.54183300 \\
\hline $\mathrm{C}$ & -0.34901200 & 5. 86110900 & 2. 90141800 \\
\hline $\mathrm{H}$ & 0.19210400 & -1.44595700 & -1.80313100 \\
\hline $\mathrm{H}$ & -2.99709400 & 0.40600900 & 0.69182700 \\
\hline $\mathrm{H}$ & -3.14786300 & -1.33695000 & 2. 35678800 \\
\hline $\mathrm{H}$ & -4.61655100 & -1.85793300 & 1. 45964900 \\
\hline $\mathrm{H}$ & -1.92218300 & -3.73670200 & -2.22868900 \\
\hline $\mathrm{H}$ & -0.19668200 & -5.28878200 & -3.11855400 \\
\hline $\mathrm{H}$ & 1.85768500 & -5.77314200 & -1.77872800 \\
\hline $\mathrm{H}$ & 2. 28697300 & -5.74725200 & 1. 28733800 \\
\hline $\mathrm{H}$ & 3. 35867000 & -4.64218200 & 0.41616000 \\
\hline $\mathrm{H}$ & 2. 68655900 & -2.75313700 & 1. 69818300 \\
\hline $\mathrm{H}$ & 2.37773900 & -3.96453200 & 2. 94991100 \\
\hline $\mathrm{H}$ & -1.29315700 & -4.30855900 & 2. 33687900 \\
\hline $\mathrm{H}$ & 0.13476300 & -5.31453100 & 2. 65519600 \\
\hline $\mathrm{H}$ & -1.13618700 & -3.94954900 & 4. 71175700 \\
\hline $\mathrm{H}$ & 0.63056300 & -4.04317300 & 4. 70233100 \\
\hline $\mathrm{H}$ & -0.65718800 & -1.49137500 & 5. 81741600 \\
\hline $\mathrm{H}$ & -0.25645400 & 0.90947600 & 5. 17533100 \\
\hline $\mathrm{H}$ & 0.45934200 & 1. 45410400 & 2. 91348800 \\
\hline $\mathrm{H}$ & 3. 24869300 & -0.18896100 & 2. 19821900 \\
\hline $\mathrm{H}$ & 6. 29402300 & -1.59846500 & -0.48942700 \\
\hline $\mathrm{H}$ & 2.30003600 & -0.86923200 & -1.92611900 \\
\hline $\mathrm{H}$ & 3. 24304000 & -0.70001700 & -3.91493700 \\
\hline $\mathrm{H}$ & 2. 89133200 & -2.40274800 & -3.53987700 \\
\hline $\mathrm{H}$ & 4. 07621400 & -1.99684900 & -4.80255100 \\
\hline $\mathrm{H}$ & 6. 28154300 & -3.22368300 & -2.05358100 \\
\hline $\mathrm{H}$ & 5. 75734400 & -3.46365500 & -3.73349700 \\
\hline $\mathrm{H}$ & 4. 65706300 & -3.86829900 & -2.39105100 \\
\hline $\mathrm{H}$ & 5. 54634200 & 0.29322000 & -3.31840900 \\
\hline $\mathrm{H}$ & 6. 29304600 & -1.01740500 & -4.26893700 \\
\hline $\mathrm{H}$ & 6.80639900 & -0.73079400 & -2.59079500 \\
\hline $\mathrm{H}$ & 5. 01848000 & 0.60627400 & 3. 40030800 \\
\hline $\mathrm{H}$ & 6. 16578200 & -0.45892500 & 4. 23854800 \\
\hline
\end{tabular}




\begin{tabular}{|c|c|c|c|}
\hline H & 4. 55030400 & -1.06195000 & 3. 81706300 \\
\hline $\mathrm{H}$ & 5. 63632100 & -3.04742300 & 2. 59552700 \\
\hline $\mathrm{H}$ & 7. 21614100 & -2.41217000 & 3. 12322100 \\
\hline $\mathrm{H}$ & 6.92982400 & -2.77104000 & 1. 40584600 \\
\hline $\mathrm{H}$ & 6.81643600 & 1. 02896600 & 1. 60774600 \\
\hline $\mathrm{H}$ & 7. 63329100 & -0.33726500 & 0.81442800 \\
\hline H & 7. 90733500 & -0.02593800 & 2. 54220900 \\
\hline $\mathrm{H}$ & -0.85534600 & 2. 32229800 & 1. 22575400 \\
\hline $\mathrm{H}$ & 1. 66284800 & 5. 77648600 & 0.90799500 \\
\hline H & 3. 25540900 & 1. 88565500 & 0.03821400 \\
\hline $\mathrm{H}$ & 4. 37953900 & 3. 02913500 & -1.55754100 \\
\hline $\mathrm{H}$ & 5. 30760500 & 4. 53864900 & -1.71506700 \\
\hline $\mathrm{H}$ & 3. 57222600 & 4. 50547500 & -2.12723800 \\
\hline $\mathrm{H}$ & 4. 98878000 & 6. 43228800 & -0.16314800 \\
\hline H & 3. 79705200 & 6. 39930100 & 1. 15188700 \\
\hline $\mathrm{H}$ & 3. 25528500 & 6.50927200 & -0.54333100 \\
\hline $\mathrm{H}$ & 5. 13714400 & 2. 86387000 & 0.93289800 \\
\hline $\mathrm{H}$ & 4. 85531900 & 4. 23845100 & 2. 02692800 \\
\hline $\mathrm{H}$ & 6.06033800 & 4. 36920000 & 0.71883500 \\
\hline $\mathrm{H}$ & -1.91254700 & 3. 57451100 & 2. 86280100 \\
\hline $\mathrm{H}$ & -2.54870100 & 3. 77597000 & 1. 19407800 \\
\hline $\mathrm{H}$ & -2.87446400 & 5. 00385100 & 2. 43420600 \\
\hline $\mathrm{H}$ & -0.36307000 & 6.65975000 & 0.19391600 \\
\hline $\mathrm{H}$ & -1.98334500 & 6. 79221000 & 0.91396700 \\
\hline $\mathrm{H}$ & -1.66933700 & 5. 54387700 & -0.31496300 \\
\hline $\mathrm{H}$ & 0.52005000 & 6. 49780900 & 2. 67430100 \\
\hline $\mathrm{H}$ & -0.05885200 & 5. 17427800 & 3. 71346700 \\
\hline $\mathrm{H}$ & -1.14797200 & 6.51776200 & 3. 28320100 \\
\hline $\mathrm{C}$ & -4.27042400 & -0.43335000 & -0.83248500 \\
\hline $\mathrm{H}$ & -4.44709100 & -1.41335300 & -1.30072600 \\
\hline $\mathrm{H}$ & -3.81660700 & 0.21016300 & -1.59662000 \\
\hline $\mathrm{C}$ & -5.56044200 & 0.20371200 & -0.35182800 \\
\hline $\mathrm{C}$ & -6.73158800 & -0.57048900 & -0.03651500 \\
\hline $\mathrm{C}$ & -5.59718400 & 1. 58341900 & -0.19991200 \\
\hline $\mathrm{C}$ & -6.80291600 & -1.98952400 & -0.17961300 \\
\hline $\mathrm{C}$ & -7.90877600 & 0.10928600 & 0.44144900 \\
\hline $\mathrm{C}$ & -6.75802300 & 2. 24165800 & 0.27149400 \\
\hline $\mathrm{H}$ & -4.71849600 & 2. 18616100 & -0.45776400 \\
\hline $\mathrm{C}$ & -7.94865700 & -2.69288000 & 0.13294800 \\
\hline $\mathrm{H}$ & -5.93609400 & -2.53956400 & -0.54879800 \\
\hline $\mathrm{C}$ & -9.07351400 & -0.64756500 & 0.75476200 \\
\hline $\mathrm{C}$ & -7.88902900 & 1. 52297800 & 0.58899500 \\
\hline $\mathrm{H}$ & -6.74573000 & 3. 32959100 & 0.37483200 \\
\hline $\mathrm{C}$ & -9.09924200 & -2.01739500 & 0.60765500 \\
\hline 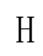 & -7.96925100 & -3.77885400 & 0.00937700 \\
\hline
\end{tabular}




$\begin{array}{rrrr}\mathrm{H} & -9.95733400 & -0.11442500 & 1.11654800 \\ \mathrm{H} & -8.78898900 & 2.02692600 & 0.95250900 \\ \mathrm{H} & -10.00221500 & -2.58270500 & 0.85183300 \\ \mathrm{C} & -0.18757100 & -0.26854600 & -4.74468900 \\ \mathrm{H} & -1.13694400 & -0.52683000 & -5.23706900 \\ \mathrm{H} & 0.31446900 & -1.18415900 & -4.40901500 \\ \mathrm{H} & 0.45888900 & 0.23616200 & -5.48288700 \\ \mathrm{C} & -0.38811400 & 0.69184300 & -3.57640300 \\ \mathrm{O} & 0.64494900 & 0.89564300 & -2.80255800 \\ \mathrm{C} & -1.27158300 & 1.90424700 & -3.89142900 \\ \mathrm{H} & -0.84613500 & 2.31841900 & -4.82532500 \\ \mathrm{H} & -2.29092500 & 1.57261500 & -4.14930400 \\ \mathrm{C} & -1.28734300 & 3.01519600 & -2.83757800 \\ \mathrm{H} & -0.24769600 & 3.22651000 & -2.53812900 \\ \mathrm{H} & -1.70561100 & 3.92940400 & -3.28074000 \\ \mathrm{C} & -2.09276300 & 2.71616400 & -1.56719400 \\ 0 & -2.94930700 & 3.52571500 & -1.19549000 \\ \mathrm{O} & -1.82117600 & 1.63219200 & -0.92743200 \\ \mathrm{H} & -1.43950300 & -0.24910100 & -2.84294000\end{array}$

\section{IntER}

$\begin{array}{rrrr}\text { Ir } & 0.67838800 & -0.52385800 & 1.10249400 \\ \text { P } & -0.87323400 & 0.20099700 & -0.41006200 \\ \text { O } & 1.76774900 & -3.92811800 & -1.24613600 \\ \mathrm{~N} & 1.52687800 & -1.96832400 & -0.19249200 \\ \mathrm{C} & 2.72790500 & -1.76620100 & -1.05216700 \\ \mathrm{C} & 2.94093500 & -3.17950800 & -1.64777300 \\ \mathrm{C} & 1.07486300 & -3.15296300 & -0.40074100 \\ \mathrm{C} & -0.16694800 & -3.70155700 & 0.19217800 \\ \mathrm{C} & -0.11528200 & -4.33342300 & 1.44466600 \\ \mathrm{C} & -1.28310000 & -4.81104300 & 2.04358700 \\ \mathrm{C} & -2.51416000 & -4.66863300 & 1.39478400 \\ \mathrm{C} & -2.56488400 & -4.04460000 & 0.14680100 \\ \mathrm{C} & -3.76032600 & -3.88065200 & -0.76150900 \\ \mathrm{C} & -3.25176100 & -2.92333900 & -1.85707700 \\ \mathrm{C} & -1.69480600 & -3.07939100 & -1.88382800 \\ \mathrm{C} & -1.40007600 & -3.54326800 & -0.45791000 \\ \mathrm{C} & -1.29190200 & -4.25031300 & -2.84221900 \\ \mathrm{C} & -1.18880300 & -3.61935000 & -4.22975300 \\ \mathrm{C} & -0.77552500 & -2.20277000 & -3.91739000 \\ \mathrm{C} & -0.24796500 & -1.30549500 & -4.84516200 \\ \mathrm{C} & 0.04676700 & -0.01052500 & -4.43230900 \\ \mathrm{C} & -0.19627100 & 0.36175000 & -3.10923200 \\ \mathrm{C} & -0.73059300 & -0.52209600 & -2.14641800\end{array}$




\begin{tabular}{|c|c|c|c|}
\hline $\mathrm{C}$ & -1.01649800 & -1.85688500 & -2.56525800 \\
\hline $\mathrm{C}$ & -2.68088100 & 0.13098100 & -0.04047200 \\
\hline $\mathrm{C}$ & -3.61604700 & 0.47833000 & -1.03051200 \\
\hline $\mathrm{C}$ & -4.99126500 & 0.47893700 & -0.75921400 \\
\hline $\mathrm{C}$ & -5.39434800 & 0.10664400 & 0.53463800 \\
\hline $\mathrm{C}$ & -4.49244900 & -0.25712200 & 1. 54669400 \\
\hline $\mathrm{C}$ & -3.12134300 & -0.23181100 & 1. 23813200 \\
\hline $\mathrm{C}$ & -5.02949100 & -0.66224800 & 2.93558800 \\
\hline $\mathrm{C}$ & -3.90056300 & -1.05114100 & 3. 90581000 \\
\hline $\mathrm{C}$ & -5.97606500 & -1.87617000 & 2. 78782800 \\
\hline $\mathrm{C}$ & -5.80767800 & 0.52479000 & 3. 54966400 \\
\hline $\mathrm{C}$ & -6.05054700 & 0.86394200 & -1.81374000 \\
\hline $\mathrm{C}$ & -5.41799800 & 1. 20908000 & -3.17415500 \\
\hline $\mathrm{C}$ & -7.02712400 & -0.31658800 & -2.02303400 \\
\hline $\mathrm{C}$ & -6.83842900 & 2. 09938000 & -1.31919400 \\
\hline $\mathrm{C}$ & -0.52745900 & 2. 01867000 & -0.61804100 \\
\hline $\mathrm{C}$ & 0.82324700 & 2. 41010000 & -0.55550300 \\
\hline $\mathrm{C}$ & 1. 20040000 & 3. 75269800 & -0.61793900 \\
\hline $\mathrm{C}$ & 0.17543900 & 4. 71104200 & -0.74990900 \\
\hline $\mathrm{C}$ & -1.17870600 & 4. 36710600 & -0.80574200 \\
\hline $\mathrm{C}$ & -1.51305400 & 2. 99964000 & -0.73435300 \\
\hline $\mathrm{C}$ & -2.30159700 & 5. 41843500 & -0.92543900 \\
\hline $\mathrm{C}$ & -3.25907000 & 5.28898300 & 0.28198100 \\
\hline $\mathrm{C}$ & -1.75582200 & 6.85776300 & -0.95117200 \\
\hline $\mathrm{C}$ & -3.08877100 & 5. 17989300 & -2.23459000 \\
\hline $\mathrm{C}$ & 2. 66930800 & 4. 20935700 & -0.51263200 \\
\hline $\mathrm{C}$ & 3. 63810300 & 3. 02456500 & -0.34550200 \\
\hline $\mathrm{C}$ & 2. 82441400 & 5. 13150900 & 0.71941700 \\
\hline $\mathrm{C}$ & 3. 06230800 & 4. 98453300 & -1.79120200 \\
\hline $\mathrm{H}$ & -0.37701500 & -1.57091200 & 1. 49541900 \\
\hline $\mathrm{H}$ & 2. 42945100 & -1.05048700 & -1.83775200 \\
\hline $\mathrm{H}$ & 2. 99653800 & -3.20546900 & -2.74362200 \\
\hline $\mathrm{H}$ & 3. 82575900 & -3.68308200 & -1.22815300 \\
\hline $\mathrm{H}$ & 0.84414300 & -4.42813000 & 1. 95751800 \\
\hline $\mathrm{H}$ & -1.22991800 & -5.29555500 & 3. 02137900 \\
\hline $\mathrm{H}$ & -3.42751800 & -5.05452900 & 1. 85603200 \\
\hline $\mathrm{H}$ & -4.05070900 & -4.86281700 & -1.17642600 \\
\hline $\mathrm{H}$ & -4.64573700 & -3.48730800 & -0.23760200 \\
\hline $\mathrm{H}$ & -3.50657900 & -1.89322700 & -1.58657800 \\
\hline $\mathrm{H}$ & -3.69521600 & -3.12135700 & -2.84473100 \\
\hline $\mathrm{H}$ & -0.30659700 & -4.63463900 & -2.54058200 \\
\hline $\mathrm{H}$ & -2.00563100 & -5.08549000 & -2.78048500 \\
\hline $\mathrm{H}$ & -0.47491200 & -4.13670300 & -4.89039200 \\
\hline $\mathrm{H}$ & -2.16169500 & -3.62144600 & -4.75415100 \\
\hline $\mathrm{H}$ & -0.07529500 & -1.62104700 & -5.87784300 \\
\hline
\end{tabular}




\begin{tabular}{|c|c|c|c|}
\hline H & 0.46258000 & 0.71964800 & -5.13114600 \\
\hline $\mathrm{H}$ & 0.03976000 & 1. 38394600 & -2.81796600 \\
\hline $\mathrm{H}$ & -3.25701300 & 0.73735200 & -2.02591800 \\
\hline $\mathrm{H}$ & -6.46182700 & 0.09687800 & 0.76296500 \\
\hline $\mathrm{H}$ & -2.37751700 & -0.49444500 & 1. 98752000 \\
\hline $\mathrm{H}$ & -3.20868900 & -0.21577000 & 4. 08803100 \\
\hline H & -3.31375400 & -1.90501700 & 3. 53332800 \\
\hline $\mathrm{H}$ & -4.33049900 & -1.34201600 & 4. 87717100 \\
\hline $\mathrm{H}$ & -6.83508400 & -1.65791100 & 2. 13481700 \\
\hline H & -6.37484600 & -2.17333400 & 3. 77161200 \\
\hline $\mathrm{H}$ & -5.44179700 & -2.74181200 & 2. 36423400 \\
\hline H & -5.15405100 & 1. 40261500 & 3. 67302100 \\
\hline $\mathrm{H}$ & -6.20121600 & 0.25237000 & 4. 54270900 \\
\hline H & -6.66267600 & 0.82732400 & 2. 92533600 \\
\hline H & -4.73491700 & 2. 06979400 & -3.10481600 \\
\hline $\mathrm{H}$ & -6.20673600 & 1. 47387600 & -3.89557500 \\
\hline H & -4.85587200 & 0.36116900 & -3.59555100 \\
\hline $\mathrm{H}$ & -6.49362200 & -1.21400800 & -2.37470300 \\
\hline $\mathrm{H}$ & -7.78753000 & -0.05653500 & -2.77746800 \\
\hline $\mathrm{H}$ & -7.55793000 & -0.58345000 & -1.09652400 \\
\hline $\mathrm{H}$ & -6.16897600 & 2. 96106600 & -1.16799600 \\
\hline $\mathrm{H}$ & -7.35323400 & 1.90495100 & -0.36593800 \\
\hline $\mathrm{H}$ & -7.60354100 & 2. 38919100 & -2.05808800 \\
\hline $\mathrm{H}$ & 1. 58834700 & 1. 64255300 & -0.43936300 \\
\hline $\mathrm{H}$ & 0.45746400 & 5. 76217100 & -0.79362800 \\
\hline $\mathrm{H}$ & -2.56192700 & 2. 70723500 & -0.74900700 \\
\hline $\mathrm{H}$ & -3.73185000 & 4. 29632900 & 0.32970500 \\
\hline $\mathrm{H}$ & -4.06393900 & 6.03955800 & 0.21535900 \\
\hline $\mathrm{H}$ & -2.72177800 & 5. 44701700 & 1. 23034800 \\
\hline $\mathrm{H}$ & -2.59133900 & 7. 57057000 & -1.03413900 \\
\hline $\mathrm{H}$ & -1.08910300 & 7. 03104500 & -1.81033200 \\
\hline $\mathrm{H}$ & -1.20202200 & 7. 10371000 & -0.03181400 \\
\hline $\mathrm{H}$ & -3.53467300 & 4. 17397700 & -2.26614900 \\
\hline $\mathrm{H}$ & -2.43341100 & 5.28314200 & -3.11424700 \\
\hline $\mathrm{H}$ & -3.90801400 & 5.91136000 & -2.33263500 \\
\hline $\mathrm{H}$ & 3.60440600 & 2. 33621300 & -1.20486000 \\
\hline $\mathrm{H}$ & 3. 43014600 & 2. 44799700 & 0.56903000 \\
\hline $\mathrm{H}$ & 4. 67190000 & 3. 39505500 & -0.26855100 \\
\hline $\mathrm{H}$ & 2. 19965400 & 6. 03472500 & 0.64092100 \\
\hline $\mathrm{H}$ & 3. 87186600 & 5. 45905500 & 0.82223800 \\
\hline $\mathrm{H}$ & 2. 53972100 & 4. 60283200 & 1. 64232600 \\
\hline $\mathrm{H}$ & 2. 42983000 & 5.87223600 & -1.94656700 \\
\hline $\mathrm{H}$ & 2. 97126900 & 4. 34603500 & -2.68470600 \\
\hline $\mathrm{H}$ & 4. 10773900 & 5. 32776100 & -1.72548200 \\
\hline$C$ & 3.92030200 & -1.21434600 & -0.27370200 \\
\hline
\end{tabular}




$\begin{array}{lrrr}\mathrm{H} & 4.16310900 & -1.92649800 & 0.53122800 \\ \mathrm{H} & 3.59114300 & -0.31955500 & 0.27314200 \\ \mathrm{C} & 5.16833300 & -0.88688400 & -1.06402100 \\ \mathrm{C} & 6.32122400 & -0.41316000 & -0.33781600 \\ \mathrm{C} & 5.25057200 & -1.01077400 & -2.44058600 \\ \mathrm{C} & 6.30867000 & -0.21616000 & 1.07530000 \\ \mathrm{C} & 7.52834100 & -0.11509400 & -1.05896400 \\ \mathrm{C} & 6.44140200 & -0.70628800 & -3.14962600 \\ \mathrm{H} & 4.38634900 & -1.34554400 & -3.01818900 \\ \mathrm{C} & 7.43574700 & 0.23775800 & 1.73341400 \\ \mathrm{H} & 5.41064700 & -0.39886400 & 1.67192600 \\ \mathrm{C} & 8.66969800 & 0.34381900 & -0.34454900 \\ \mathrm{C} & 7.55837900 & -0.27582000 & -2.47397700 \\ \mathrm{H} & 6.46203300 & -0.82062700 & -4.23659000 \\ \mathrm{C} & 8.62795400 & 0.51544100 & 1.02336400 \\ \mathrm{H} & 7.39424300 & 0.38510100 & 2.81540400 \\ \mathrm{H} & 9.58371600 & 0.56284900 & -0.90402700 \\ \mathrm{H} & 8.48205600 & -0.04545800 & -3.01212000 \\ \mathrm{H} & 9.51188300 & 0.87081700 & 1.55977500 \\ \mathrm{C} & -1.10979200 & 2.15870700 & 3.93388900 \\ \mathrm{H} & -1.48679800 & 2.15046000 & 4.97031000 \\ \mathrm{H} & -1.96733300 & 2.19961100 & 3.24471500 \\ \mathrm{H} & -0.51906600 & 3.07743100 & 3.78507900 \\ \mathrm{C} & -0.24503500 & 0.92850000 & 3.62665300 \\ \mathrm{O} & 0.13782700 & 1.01225900 & 2.28307500 \\ \mathrm{C} & 0.93249900 & 0.84435000 & 4.61543700 \\ \mathrm{H} & 1.58857800 & 1.71968700 & 4.46224100 \\ \mathrm{H} & 0.52056700 & 0.93819100 & 5.63593900 \\ \mathrm{C} & 1.77200900 & -0.44469300 & 4.56713000 \\ \mathrm{H} & 2.35272000 & -0.52982400 & 5.49701900 \\ \mathrm{C} & 1.10215600 & -1.32019200 & 4.50189300 \\ \mathrm{H} & 2.78878700 & -0.49463900 & 3.42936900 \\ \mathrm{H} & 3.97005300 & -0.25433200 & 3.63730000 \\ & 2.35725300 & -0.83297700 & 2.23883900 \\ \mathrm{H} & -0.02332400 & 3.80246200\end{array}$

\section{IntAS}

$\begin{array}{rrrr}\text { Ir } & 0.71946000 & 0.20678900 & -1.16537300 \\ \mathrm{P} & -1.09286100 & -0.56750300 & -0.07115500 \\ 0 & 2.97449900 & -0.55283400 & 2.54912800 \\ \mathrm{~N} & 2.18401600 & -0.25917300 & 0.47485300 \\ \mathrm{C} & 3.26727400 & -1.25769100 & 0.32559800 \\ \mathrm{C} & 3.56146600 & -1.63957900 & 1.79098300 \\ \mathrm{C} & 2.14283600 & 0.09584700 & 1.70829400\end{array}$




\begin{tabular}{|c|c|c|c|}
\hline C & 1. 30050800 & 1. 16617300 & 2. 30071600 \\
\hline $\mathrm{C}$ & 1. 65169100 & 2.50681900 & 2. 07132700 \\
\hline $\mathrm{C}$ & 0.92864900 & 3.54288700 & 2. 66559100 \\
\hline $\mathrm{C}$ & -0.17470200 & 3. 26023300 & 3. 47751200 \\
\hline $\mathrm{C}$ & -0.54962000 & 1. 93227600 & 3. 68025900 \\
\hline $\mathrm{C}$ & -1.69248400 & 1. 39604200 & 4. 51229900 \\
\hline $\mathrm{C}$ & -1.77387100 & -0.08752600 & 4. 09572600 \\
\hline $\mathrm{C}$ & -0.34565600 & -0.45986800 & 3. 58744000 \\
\hline $\mathrm{C}$ & 0.18453000 & 0.88345200 & 3. 10511500 \\
\hline $\mathrm{C}$ & 0.53054800 & -0.95495400 & 4. 78844300 \\
\hline $\mathrm{C}$ & 0.32178500 & -2.47085900 & 4. 83457600 \\
\hline $\mathrm{C}$ & 0.10128000 & -2.80899900 & 3. 38038300 \\
\hline $\mathrm{C}$ & 0.29493600 & -4.05539400 & 2. 79369300 \\
\hline $\mathrm{C}$ & 0.09373700 & -4.18354700 & 1. 41813900 \\
\hline $\mathrm{C}$ & -0.34250100 & -3.08639200 & 0.67959200 \\
\hline $\mathrm{C}$ & -0.60794600 & -1.82116400 & 1. 25854800 \\
\hline $\mathrm{C}$ & -0.31657400 & -1.67487400 & 2. 63854400 \\
\hline $\mathrm{C}$ & -2.27013000 & 0.73597600 & 0.54572700 \\
\hline C & -3.50344300 & 0.40929800 & 1. 13442400 \\
\hline C & -4.45286400 & 1. 39851100 & 1. 42515700 \\
\hline $\mathrm{C}$ & -4.13935700 & 2. 72188800 & 1. 07012700 \\
\hline $\mathrm{C}$ & -2.93118400 & 3. 08549300 & 0.45954500 \\
\hline $\mathrm{C}$ & -1.98867600 & 2. 07007000 & 0.21916300 \\
\hline $\mathrm{C}$ & -2.70167200 & 4. 55272200 & 0.03834700 \\
\hline $\mathrm{C}$ & -1.31090200 & 4. 76588400 & -0.58352400 \\
\hline $\mathrm{C}$ & -2.83239500 & 5. 48116900 & 1. 26714500 \\
\hline $\mathrm{C}$ & -3.76675200 & 4. 94561000 & -1.01285800 \\
\hline $\mathrm{C}$ & -5.82226400 & 1. 08598300 & 2. 06508000 \\
\hline $\mathrm{C}$ & -5.95546700 & -0.39415600 & 2. 46431700 \\
\hline $\mathrm{C}$ & -6.01580400 & 1. 94270600 & 3. 33754000 \\
\hline $\mathrm{C}$ & -6.94324100 & 1. 41581600 & 1. 05157300 \\
\hline $\mathrm{C}$ & -2.30406000 & -1.56859400 & -1.09632600 \\
\hline $\mathrm{C}$ & -2.43567700 & -1.25251800 & -2.45822200 \\
\hline $\mathrm{C}$ & -3.36021800 & -1.91568800 & -3.27385000 \\
\hline $\mathrm{C}$ & -4.14425100 & -2.92922000 & -2.69317400 \\
\hline $\mathrm{C}$ & -4.03995500 & -3.28145800 & -1.34210800 \\
\hline $\mathrm{C}$ & -3.11932800 & -2.56899400 & -0.55100700 \\
\hline $\mathrm{C}$ & -4.85958100 & -4.43206200 & -0.71831800 \\
\hline $\mathrm{C}$ & -5.68318200 & -3.90727200 & 0.47869800 \\
\hline $\mathrm{C}$ & -5.83708600 & -5.06648300 & -1.72460900 \\
\hline $\mathrm{C}$ & -3.89185200 & -5.53460400 & -0.22764300 \\
\hline $\mathrm{C}$ & -3.52537900 & -1.58594000 & -4.77192600 \\
\hline $\mathrm{C}$ & -2.59647900 & -0.44366200 & -5.22143200 \\
\hline $\mathrm{C}$ & -4.98553400 & -1.15641200 & -5.04575500 \\
\hline $\mathrm{C}$ & -3.19265300 & -2.83929700 & -5.61458700 \\
\hline
\end{tabular}




\begin{tabular}{|c|c|c|c|}
\hline H & -0.22278000 & 0.54156500 & -2.39094500 \\
\hline $\mathrm{H}$ & 2.87648600 & -2.11708900 & -0.23645000 \\
\hline $\mathrm{H}$ & 3. 05602400 & -2.56959600 & 2. 09466900 \\
\hline $\mathrm{H}$ & 4. 62723700 & -1.70083600 & 2. 04317300 \\
\hline H & 2. 49277200 & 2. 73507300 & 1. 41709100 \\
\hline $\mathrm{H}$ & 1. 22614100 & 4. 57844800 & 2. 48466700 \\
\hline $\mathrm{H}$ & -0.74088800 & 4. 07213500 & 3. 94210600 \\
\hline $\mathrm{H}$ & -1.46764900 & 1.50710800 & 5. 58856200 \\
\hline $\mathrm{H}$ & -2.63489500 & 1. 93601000 & 4. 32895800 \\
\hline H & -2.49078100 & -0.19545900 & 3. 27420400 \\
\hline $\mathrm{H}$ & -2.10286300 & -0.74985600 & 4. 91089700 \\
\hline H & 1. 58649900 & -0.74075900 & 4. 57281000 \\
\hline H & 0.26636100 & -0.43849800 & 5. 72358100 \\
\hline $\mathrm{H}$ & 1. 17229200 & -3.01373800 & 5. 27629100 \\
\hline H & -0.57063700 & -2.74132600 & 5. 42849500 \\
\hline $\mathrm{H}$ & 0.62294300 & -4.90683800 & 3. 39636200 \\
\hline H & 0.27610000 & -5.13603500 & 0.91404300 \\
\hline $\mathrm{H}$ & -0.48336600 & -3.21831100 & -0.39343900 \\
\hline $\mathrm{H}$ & -3.73181100 & -0.63438700 & 1. 34312000 \\
\hline H & -4.88130700 & 3. 49962500 & 1. 26162500 \\
\hline $\mathrm{H}$ & -1.03286700 & 2. 30420200 & -0.24852900 \\
\hline H & -1.16988500 & 4. 16783400 & -1.49557100 \\
\hline $\mathrm{H}$ & -0.50121100 & 4. 49348000 & 0.10833800 \\
\hline $\mathrm{H}$ & -1.18787000 & 5. 82715100 & -0.85465600 \\
\hline $\mathrm{H}$ & -3.82597000 & 5. 41338300 & 1. 73635000 \\
\hline $\mathrm{H}$ & -2.67788200 & 6.53201200 & 0.97133700 \\
\hline $\mathrm{H}$ & -2.08079600 & 5. 22903000 & 2. 03102700 \\
\hline $\mathrm{H}$ & -3.69869800 & 4. 29878200 & -1.90182000 \\
\hline $\mathrm{H}$ & -3.61839500 & 5.98858800 & -1.33897900 \\
\hline H & -4.79026800 & 4. 86346100 & -0.61521400 \\
\hline $\mathrm{H}$ & -5.88633600 & -1.06434400 & 1. 59512700 \\
\hline $\mathrm{H}$ & -6.93607300 & -0.56683200 & 2. 93531500 \\
\hline $\mathrm{H}$ & -5.18278200 & -0.69461300 & 3. 18958800 \\
\hline $\mathrm{H}$ & -5.23461900 & 1. 72590500 & 4. 08369700 \\
\hline $\mathrm{H}$ & -6.99337600 & 1. 72799400 & 3. 79976300 \\
\hline $\mathrm{H}$ & -5.98309100 & 3. 02118900 & 3. 12148500 \\
\hline $\mathrm{H}$ & -6.83493300 & 0.81430200 & 0.13523300 \\
\hline $\mathrm{H}$ & -6.93053700 & 2. 47664800 & 0.75809200 \\
\hline $\mathrm{H}$ & -7.93339100 & 1. 20075000 & 1. 48683700 \\
\hline $\mathrm{H}$ & -1.78606300 & -0.48488400 & -2.87134500 \\
\hline $\mathrm{H}$ & -4.85028000 & -3.46367700 & -3.32710300 \\
\hline $\mathrm{H}$ & -3.01685700 & -2.83013200 & 0.50298100 \\
\hline $\mathrm{H}$ & -5.04321400 & -3.48651600 & 1. 26864800 \\
\hline $\mathrm{H}$ & -6.26932900 & -4.72463100 & 0.93028400 \\
\hline 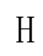 & -6.38716200 & -3.12216400 & 0. 15987900 \\
\hline
\end{tabular}




\begin{tabular}{|c|c|c|c|}
\hline H & -6.40560200 & -5.87183900 & -1.23327100 \\
\hline $\mathrm{H}$ & -5.31309700 & -5.51151300 & -2.58455100 \\
\hline $\mathrm{H}$ & -6.56475800 & -4.33451800 & -2.10877100 \\
\hline $\mathrm{H}$ & -3.18947000 & -5.15779200 & 0.53147300 \\
\hline H & -3.29675200 & -5.93586500 & -1.06343400 \\
\hline $\mathrm{H}$ & -4.45484500 & -6.36989500 & 0.22125300 \\
\hline $\mathrm{H}$ & -1.53401800 & -0.69426600 & -5.08081900 \\
\hline H & -2.79813900 & 0.48945100 & -4.67297300 \\
\hline $\mathrm{H}$ & -2.75093200 & -0.23930600 & -6.29278000 \\
\hline H & -5.70484200 & -1.95036500 & -4.79234200 \\
\hline $\mathrm{H}$ & -5.12152200 & -0.91404400 & -6.11278100 \\
\hline H & -5.25052700 & -0.26313300 & -4.45795300 \\
\hline H & -3.85462100 & -3.68561800 & -5.37454100 \\
\hline $\mathrm{H}$ & -2.15459500 & -3.16510400 & -5.44234900 \\
\hline H & -3.30705100 & -2.62208100 & -6.68950900 \\
\hline $\mathrm{C}$ & 4. 45451000 & -0.64890600 & -0.45474800 \\
\hline $\mathrm{H}$ & 4. 84352200 & 0.21024200 & 0.11123400 \\
\hline $\mathrm{H}$ & 4. 04399900 & -0.23548700 & -1.38745000 \\
\hline $\mathrm{C}$ & 5.54285000 & -1.65442900 & -0.77420600 \\
\hline $\mathrm{C}$ & 6. 76973200 & -1.74358100 & -0.02829400 \\
\hline $\mathrm{C}$ & 5. 33373200 & -2.53733300 & -1.82364400 \\
\hline $\mathrm{C}$ & 7. 09699000 & -0.87104000 & 1. 05302400 \\
\hline $\mathrm{C}$ & 7. 73327500 & -2.75371000 & -0.38538600 \\
\hline $\mathrm{C}$ & 6.27997300 & -3.52925100 & -2.17036900 \\
\hline $\mathrm{H}$ & 4. 41115400 & -2.45661800 & -2.40590500 \\
\hline $\mathrm{C}$ & 8. 28662200 & -0.98852100 & 1. 74220500 \\
\hline $\mathrm{H}$ & 6. 39895500 & -0.08420400 & 1. 34044500 \\
\hline $\mathrm{C}$ & 8.95061900 & -2.84698100 & 0.34768000 \\
\hline $\mathrm{C}$ & 7. 45681100 & -3.63804800 & -1.46335700 \\
\hline $\mathrm{H}$ & 6. 07271300 & -4.20287000 & -3.00573700 \\
\hline $\mathrm{C}$ & 9. 22558800 & -1.98839000 & 1. 38942700 \\
\hline $\mathrm{H}$ & 8.50816600 & -0.30143500 & 2. 56285900 \\
\hline $\mathrm{H}$ & 9.67087400 & -3.61930400 & 0.06349700 \\
\hline $\mathrm{H}$ & 8. 19666700 & -4.39993100 & -1.72338200 \\
\hline $\mathrm{H}$ & 10.16534900 & -2.07256900 & 1. 94095600 \\
\hline $\mathrm{C}$ & 2. 83891600 & 7. 24446900 & -2.98517500 \\
\hline $\mathrm{H}$ & 1. 86710300 & 7. 45534200 & -2.51031900 \\
\hline $\mathrm{H}$ & 2. 65803200 & 7. 18826100 & -4.07255900 \\
\hline $\mathrm{H}$ & 3.54805200 & 8. 05774100 & -2.78187100 \\
\hline $\mathrm{C}$ & 3. 41205600 & 5. 92364600 & -2.50413200 \\
\hline 0 & 4. 57129300 & 5. 81868900 & -2.16639000 \\
\hline $\mathrm{C}$ & 2. 44414700 & 4. 75035300 & -2.45252600 \\
\hline $\mathrm{H}$ & 1. 78532800 & 4. 91800900 & -1.58036700 \\
\hline $\mathrm{H}$ & 1. 77231300 & 4. 78604900 & -3.32757400 \\
\hline $\mathrm{C}$ & 3. 12223600 & 3. 39161700 & -2.31536800 \\
\hline
\end{tabular}




$\begin{array}{rrrr}\mathrm{H} & 3.59433200 & 3.07162900 & -3.25560600 \\ \mathrm{H} & 3.93944800 & 3.48012900 & -1.57874500 \\ \mathrm{C} & 2.19013500 & 2.29367700 & -1.84393400 \\ 0 & 1.26987900 & 2.52947400 & -1.01702200 \\ 0 & 2.37350300 & 1.10758900 & -2.26761000 \\ \mathrm{H} & 0.76561200 & -1.26991900 & -1.67737100\end{array}$

\section{IntBS}

Ir

$\mathrm{P}$

0

N

C

C

C

C

C

C

C

C

C

C

C

C

C

C

C

C

C

C

C

C

C

C

C

C

C

C

C

C

C

C

$\mathrm{C}$

C
0. 67958000

$-0.99377000$

3. 03282000

2. 26837600

3. 48784500

3. 75388300

2. 14100000

1. 14429100

1. 23444700

0. 36117700

$-0.62244600$

$-0.74936800$

$-1.72559900$

$-1.60065900$

$-0.16598700$

0. 11866700

0. 82815100

0. 76200300

0. 43649000

0. 58222400

0. 21075900

$-0.29004000$

$-0.44757900$

$-0.06653100$

$-2.39963900$

$-3.41569700$

$-4.56335300$

$-4.67374400$

$-3.68636500$

$-2.52894600$

$-3.83229800$

$-3.65484400$

$-2.74969100$

$-5.21390700$

$-5.70074400$

$-5.40861400$
0. 57990400

1. 08365700

0. 36698200

$-2.10125000$

$-0.62365800$

$-0.00377700$

$-0.88602400$

$-1.78683200$

$-2.81972700$

$-3.45359800$

$-4.48935100$

$-4.89900900$

$-4.23567000$

$-4.51470500$

$-3.26747000$

$-2.69737900$

$-3.18930000$

$-3.37299800$

$-2.51039200$

$-1.14207500$

0. 04846500

1. 23924900

1. 21302900

0. 02753500

$-1.19607700$

$-0.77575100$

$-0.99981700$

$-1.72826000$

$-2.20751500$

$-2.00421100$

$-1.29241600$

$-2.50728400$

$-1.31559500$

$-3.56959900$

$-3.13447600$

$-2.00752400$

$-1.41231200$
$-0.42751700$

$-1.48858800$

0. 00858400

$-0.56019600$

$-1.79213800$

$-0.52245900$

$-0.17241200$

1. 07527400

1. 41048000

0. 50781900

$-0.71412600$

$-1.83280400$

$-2.72848800$

-2. 46943900

$-1.05800000$

$-3.46950400$

$-4.73029800$

$-4.18266400$

$-4.89082200$

$-4.27515100$

$-2.97448300$

$-2.22188400$

$-2.85741800$

$-0.06499900$

$-1.01662800$

$-0.68666500$

0. 63540100

1. 60549200

1. 22613900

3. 05653400

4. 02680800

3. 35251400

3. 31951000

$-1.69415800$

$-3.08381400$ 


\begin{tabular}{|c|c|c|c|}
\hline $\mathrm{C}$ & -5.88643600 & -3.53428300 & -1.85586400 \\
\hline $\mathrm{C}$ & -7.01965100 & -1.39104300 & -1.17210900 \\
\hline $\mathrm{C}$ & -1.89112400 & 2. 03222200 & -0.52862300 \\
\hline $\mathrm{C}$ & -1.13498100 & 3. 22283700 & -0.49532700 \\
\hline $\mathrm{C}$ & -1.73770400 & 4. 48212100 & -0.50021300 \\
\hline $\mathrm{C}$ & -3.14505400 & 4. 53958000 & -0.51311200 \\
\hline $\mathrm{C}$ & -3.93642200 & 3. 38969200 & -0.52501200 \\
\hline $\mathrm{C}$ & -3.28437200 & 2. 13821100 & -0.53950100 \\
\hline $\mathrm{C}$ & -5.47876000 & 3. 44372700 & -0.52202800 \\
\hline $\mathrm{C}$ & -6.02533500 & 2. 63898400 & 0.67983600 \\
\hline $\mathrm{C}$ & -6.01398700 & 4. 88331700 & -0.41334800 \\
\hline $\mathrm{C}$ & -6.01184700 & 2. 83220900 & -1.83813800 \\
\hline $\mathrm{C}$ & -0.92083300 & 5. 79085100 & -0.46530900 \\
\hline $\mathrm{C}$ & 0.59668900 & 5. 53146300 & -0.46551900 \\
\hline $\mathrm{C}$ & -1.27339900 & 6.58102300 & 0.81658400 \\
\hline $\mathrm{C}$ & -1.26332200 & 6.64748700 & -1.70602500 \\
\hline $\mathrm{H}$ & 3. 25754400 & 1. 02949500 & -0.85261700 \\
\hline $\mathrm{H}$ & 3. 32492500 & -0.46125900 & -2.71399400 \\
\hline $\mathrm{H}$ & 4. 80830900 & -1.13798900 & -1.95900300 \\
\hline $\mathrm{H}$ & 1. 97917600 & -3.13183300 & 1. 80157400 \\
\hline $\mathrm{H}$ & 0.44184400 & -4.94214400 & 2. 39961800 \\
\hline $\mathrm{H}$ & -1.30210500 & -5.71658900 & 0.76426200 \\
\hline $\mathrm{H}$ & -1.43653100 & -5.43569800 & -2.37154700 \\
\hline $\mathrm{H}$ & -2.75426300 & -4.67186600 & -1.47122500 \\
\hline $\mathrm{H}$ & -2.34449300 & -2.52856700 & -2.41712800 \\
\hline $\mathrm{H}$ & -1.76888400 & -3.47655600 & -3.79590600 \\
\hline $\mathrm{H}$ & 1. 84176100 & -3.34359900 & -3.04994400 \\
\hline $\mathrm{H}$ & 0.56712900 & -4.42832300 & -3.63982600 \\
\hline $\mathrm{H}$ & 1. 69538200 & -2.52050900 & -5.31598600 \\
\hline $\mathrm{H}$ & -0.04112100 & -2.84419400 & -5.41262500 \\
\hline $\mathrm{H}$ & 0.97765700 & 0.03838400 & -5.91044700 \\
\hline $\mathrm{H}$ & 0.30625800 & 2. 19481600 & -4.79714700 \\
\hline $\mathrm{H}$ & -0.57101700 & 2. 16466800 & -2.53117100 \\
\hline $\mathrm{H}$ & -3.30266100 & -0.57962300 & -2.01562000 \\
\hline $\mathrm{H}$ & -5.57338600 & -2.75793600 & 0.90758100 \\
\hline $\mathrm{H}$ & -1.71013300 & -1.14473500 & 1.93311600 \\
\hline $\mathrm{H}$ & -4.41235700 & -0.53607500 & 3. 84358900 \\
\hline $\mathrm{H}$ & -2.66186400 & -0.85440100 & 3. 92525200 \\
\hline $\mathrm{H}$ & -3.76071900 & -1.65575000 & 5. 07018400 \\
\hline $\mathrm{H}$ & -2.85756500 & -4.43313000 & 2. 67711500 \\
\hline $\mathrm{H}$ & -2.85187600 & -3.93502600 & 4. 38807000 \\
\hline $\mathrm{H}$ & -1.72877700 & -3.17645700 & 3. 24100600 \\
\hline $\mathrm{H}$ & -6.03534700 & -2.42351000 & 3. 13466800 \\
\hline $\mathrm{H}$ & -5.28103900 & -3.45106900 & 4. 37214700 \\
\hline $\mathrm{H}$ & -5.38731300 & -4.02829700 & 2. 69944500 \\
\hline
\end{tabular}




\begin{tabular}{|c|c|c|c|}
\hline H & -5.30239500 & -0.31713300 & -3.05011200 \\
\hline $\mathrm{H}$ & -6.23919400 & -1.64075300 & -3.77017500 \\
\hline $\mathrm{H}$ & -4.49065900 & -1.82914900 & -3.52588800 \\
\hline $\mathrm{H}$ & -4.96944300 & -4.00654800 & -2.24174200 \\
\hline H & -6.70241900 & -3.74939100 & -2.56547000 \\
\hline $\mathrm{H}$ & -6.13894900 & -4.02134900 & -0.90181200 \\
\hline $\mathrm{H}$ & -6.92789000 & -0.30042900 & -1.05071800 \\
\hline $\mathrm{H}$ & -7.31277800 & -1.81221200 & -0.19860400 \\
\hline $\mathrm{H}$ & -7.84223000 & -1.58552600 & -1.88016000 \\
\hline H & -0.05322200 & 3. 14461600 & -0.43903700 \\
\hline $\mathrm{H}$ & -3.62642800 & 5.51652500 & -0.50046800 \\
\hline H & -3.89077000 & 1. 23605800 & -0.53581900 \\
\hline H & -5.72504100 & 1. 58132300 & 0.64223400 \\
\hline $\mathrm{H}$ & -7.12740400 & 2. 67170400 & 0.69422600 \\
\hline H & -5.66042600 & 3. 05560000 & 1. 63218900 \\
\hline $\mathrm{H}$ & -7.11527800 & 4. 87016500 & -0.40165900 \\
\hline H & -5.70278900 & 5. 50631100 & -1.26640400 \\
\hline $\mathrm{H}$ & -5.67880000 & 5. 37676000 & 0.51238800 \\
\hline $\mathrm{H}$ & -5.68626300 & 1. 78896200 & -1.96334900 \\
\hline H & -5.65403300 & 3. 40059300 & -2.71147200 \\
\hline $\mathrm{H}$ & -7.11440800 & 2.84346300 & -1.85247600 \\
\hline H & 0.92147700 & 4. 99143000 & -1.36862500 \\
\hline $\mathrm{H}$ & 0.91507200 & 4. 94733700 & 0.41151300 \\
\hline $\mathrm{H}$ & 1. 13731400 & 6. 49099300 & -0.44156800 \\
\hline $\mathrm{H}$ & -2.34130900 & 6.84471900 & 0.85715600 \\
\hline $\mathrm{H}$ & -0.69684200 & 7. 51992200 & 0.86170300 \\
\hline $\mathrm{H}$ & -1.03929200 & 5.99268600 & 1. 71791500 \\
\hline $\mathrm{H}$ & -2.33233500 & 6.90644200 & -1.74669800 \\
\hline $\mathrm{H}$ & -1.01290500 & 6. 11283100 & -2.63628700 \\
\hline $\mathrm{H}$ & -0.69319000 & 7. 59126200 & -1.69254700 \\
\hline $\mathrm{C}$ & 4. 62046900 & 0.01159600 & 0.49191700 \\
\hline $\mathrm{H}$ & 4. 81793200 & -1.02079500 & 0.81419100 \\
\hline $\mathrm{H}$ & 4. 21906100 & 0.53777300 & 1. 36802700 \\
\hline $\mathrm{C}$ & 5.88222200 & 0.70662700 & 0.02060100 \\
\hline $\mathrm{C}$ & 7. 04787700 & -0.01070900 & -0.42278700 \\
\hline $\mathrm{C}$ & 5.90450000 & 2. 09367500 & 0.00189000 \\
\hline $\mathrm{C}$ & 7. 13581300 & -1.43543700 & -0.43405300 \\
\hline $\mathrm{C}$ & 8. 19600300 & 0.73297600 & -0.87532400 \\
\hline $\mathrm{C}$ & 7. 03111400 & 2. 82119900 & -0.44692800 \\
\hline $\mathrm{H}$ & 5. 02365500 & 2. 64138200 & 0.35004700 \\
\hline $\mathrm{C}$ & 8.27455000 & -2.08489200 & -0.86473400 \\
\hline $\mathrm{H}$ & 6.28865000 & -2.03214100 & -0.09406800 \\
\hline $\mathrm{C}$ & 9. 35478400 & 0.03006600 & -1.31240300 \\
\hline $\mathrm{C}$ & 8. 15594300 & 2. 15397600 & -0.87838700 \\
\hline$U$ & 7. 00359600 & 3. 91384600 & -0.44458100 \\
\hline
\end{tabular}




$\begin{array}{lrrr}\mathrm{C} & 9.39833400 & -1.34690200 & -1.31005000 \\ \mathrm{H} & 8.31011000 & -3.17723500 & -0.85946000 \\ \mathrm{H} & 10.21754900 & 0.60968900 & -1.65242800 \\ \mathrm{H} & 9.03374200 & 2.70557500 & -1.22570800 \\ \mathrm{H} & 10.29554300 & -1.87133200 & -1.64820000 \\ \mathrm{C} & 3.43284400 & 1.79948600 & 4.44990500 \\ \mathrm{H} & 3.25839500 & 2.81479000 & 4.84552700 \\ \mathrm{H} & 4.31262800 & 1.80916200 & 3.79396400 \\ \mathrm{H} & 3.61530800 & 1.14876200 & 5.32060900 \\ \mathrm{C} & 2.20447800 & 1.34036100 & 3.71110400 \\ \mathrm{O} & 2.27015600 & 1.15814100 & 2.49347300 \\ \mathrm{C} & 0.95102000 & 1.08941700 & 4.49574000 \\ \mathrm{H} & 0.95683600 & 1.71845800 & 5.40036400 \\ \mathrm{H} & 0.09137100 & 1.35961100 & 3.86535400 \\ \mathrm{C} & 0.83606800 & -0.39428000 & 4.90471200 \\ \mathrm{H} & 1.71291900 & -0.72312300 & 5.48602600 \\ \mathrm{H} & -0.02404100 & -0.49658600 & 5.58755000 \\ \mathrm{C} & 0.61513000 & -1.42877400 & 3.77047700 \\ \mathrm{O} & 0.73590800 & -2.60957300 & 4.09675300 \\ \mathrm{O} & 0.26162100 & -1.00383600 & 2.61379900 \\ \mathrm{H} & -0.35250300 & 1.53784300 & 1.80381800 \\ \mathrm{H} & 1.12278100 & 1.74980900 & 0.13246900\end{array}$

\section{IntCS}

$\begin{array}{rrrr}\text { Ir } & 0.94490600 & 0.60260200 & 1.27721100 \\ \mathrm{P} & -0.90647700 & 0.20705300 & -0.49884500 \\ 0 & 3.14535900 & -2.20704900 & -0.99255700 \\ \mathrm{~N} & 2.29292400 & -0.57832000 & 0.26255400 \\ \mathrm{C} & 3.56489900 & -0.02397600 & -0.28513000 \\ \mathrm{C} & 3.86648600 & -1.02355000 & -1.40676800 \\ \mathrm{C} & 2.19755000 & -1.81017800 & -0.12702700 \\ \mathrm{C} & 1.21022800 & -2.82349500 & 0.28469700 \\ \mathrm{C} & 1.27347500 & -3.33869000 & 1.58857300 \\ \mathrm{C} & 0.40838000 & -4.35675700 & 1.98905700 \\ \mathrm{C} & -0.54534500 & -4.85554900 & 1.09965900 \\ \mathrm{C} & -0.63676100 & -4.31915800 & -0.18592900 \\ \mathrm{C} & -1.57286800 & -4.72234700 & -1.30097700 \\ \mathrm{C} & -1.39773500 & -3.59440300 & -2.33670400 \\ \mathrm{C} & 0.02620400 & -2.99417000 & -2.08097200 \\ \mathrm{C} & 0.23619500 & -3.30450300 & -0.60513200 \\ \mathrm{C} & 1.07300900 & -3.79014900 & -2.92770800 \\ \mathrm{C} & 1.07459000 & -3.10790500 & -4.29713400 \\ \mathrm{C} & 0.69840100 & -1.68425100 & -3.96518400 \\ \mathrm{C} & 0.83178600 & -0.60284000 & -4.83374000\end{array}$




\begin{tabular}{|c|c|c|c|}
\hline $\mathrm{C}$ & 0.38108400 & 0.64537700 & -4.41618600 \\
\hline $\mathrm{C}$ & -0.16789900 & 0.79097200 & -3.14203200 \\
\hline $\mathrm{C}$ & -0.29311800 & -0.27561600 & -2.22494500 \\
\hline $\mathrm{C}$ & 0.14369100 & -1.56274000 & -2.66666600 \\
\hline $\mathrm{C}$ & -2.43684500 & -0.80174600 & -0.25340700 \\
\hline $\mathrm{C}$ & -3.35094300 & -0.93215600 & -1.31904300 \\
\hline $\mathrm{C}$ & -4.57208700 & -1.58759200 & -1.14480100 \\
\hline $\mathrm{C}$ & -4.86499500 & -2.08350000 & 0.14214100 \\
\hline $\mathrm{C}$ & -3.99138200 & -1.96366100 & 1. 22957600 \\
\hline $\mathrm{C}$ & -2.74913000 & -1.32632600 & 1. 00453900 \\
\hline $\mathrm{C}$ & -4.36159400 & -2.49264700 & 2. 63217200 \\
\hline $\mathrm{C}$ & -4.22373500 & -1.35175400 & 3. 66658600 \\
\hline $\mathrm{C}$ & -3.40942100 & -3.64533900 & 3. 02364300 \\
\hline $\mathrm{C}$ & -5.80950900 & -3.01549300 & 2. 68860500 \\
\hline $\mathrm{C}$ & -5.58277300 & -1.79585400 & -2.29265500 \\
\hline $\mathrm{C}$ & -5.13205600 & -1.11172200 & -3.59625700 \\
\hline $\mathrm{C}$ & -5.73111200 & -3.31107800 & -2.56620100 \\
\hline $\mathrm{C}$ & -6.95987500 & -1.21457600 & -1.89792700 \\
\hline C & -1.62975200 & 1.92523200 & -0.69203000 \\
\hline $\mathrm{C}$ & -0.77224900 & 3. 02222900 & -0.91747100 \\
\hline $\mathrm{C}$ & -1.25436400 & 4. 33157800 & -0.97177900 \\
\hline $\mathrm{C}$ & -2.63075900 & 4. 53732000 & -0.75009700 \\
\hline $\mathrm{C}$ & -3.50992600 & 3. 48512100 & -0.48690800 \\
\hline $\mathrm{C}$ & -2.98593500 & 2. 17620700 & -0.47095200 \\
\hline $\mathrm{C}$ & -5.01268000 & 3. 70266300 & -0.21397100 \\
\hline $\mathrm{C}$ & -5.37047900 & 3. 13261800 & 1. 17840400 \\
\hline $\mathrm{C}$ & -5.40282900 & 5. 19151800 & -0.23523000 \\
\hline $\mathrm{C}$ & -5.83850400 & 2. 97237200 & -1.29762400 \\
\hline $\mathrm{C}$ & -0.33763000 & 5.54499100 & -1.23529600 \\
\hline $\mathrm{C}$ & 1. 12197300 & 5. 12937300 & -1.49244300 \\
\hline $\mathrm{C}$ & -0.36288100 & 6.48233000 & -0.00536100 \\
\hline $\mathrm{C}$ & -0.84093800 & 6.31661000 & -2.47732800 \\
\hline $\mathrm{H}$ & 3. 36818900 & 0.97786100 & -0.68426300 \\
\hline $\mathrm{H}$ & 3. 45324700 & -0.70409100 & -2.37676600 \\
\hline $\mathrm{H}$ & 4. 92522500 & -1.28337900 & -1.52053300 \\
\hline $\mathrm{H}$ & 1. 99774900 & -2.93211400 & 2. 29250500 \\
\hline $\mathrm{H}$ & 0.45800600 & -4.72714700 & 3. 01399800 \\
\hline $\mathrm{H}$ & -1.22748100 & -5.64959000 & 1. 41433000 \\
\hline $\mathrm{H}$ & -1.27956400 & -5.70591900 & -1.71117900 \\
\hline $\mathrm{H}$ & -2.61693100 & -4.82048800 & -0.96357100 \\
\hline $\mathrm{H}$ & -2.15282700 & -2.82118700 & -2.16191300 \\
\hline $\mathrm{H}$ & -1.51211000 & -3.93679400 & -3.37651300 \\
\hline $\mathrm{H}$ & 2. 06277100 & -3.69427600 & -2.46247100 \\
\hline $\mathrm{H}$ & 0.82529000 & -4.86148200 & -2.96604800 \\
\hline $\mathrm{H}$ & 2. 04272700 & -3.17953800 & -4.81880100 \\
\hline
\end{tabular}




\begin{tabular}{|c|c|c|c|}
\hline H & 0.32061800 & -3.54822100 & -4.97474300 \\
\hline $\mathrm{H}$ & 1. 26848000 & -0.74307800 & -5.82651100 \\
\hline $\mathrm{H}$ & 0.44895000 & 1. 51457000 & -5.07555700 \\
\hline $\mathrm{H}$ & -0.51860800 & 1. 77990400 & -2.85908700 \\
\hline H & -3.09216700 & -0.50604300 & -2.28788300 \\
\hline $\mathrm{H}$ & -5.82079800 & -2.58295700 & 0.29215700 \\
\hline $\mathrm{H}$ & -2.01790400 & -1.23748300 & 1. 80848800 \\
\hline $\mathrm{H}$ & -4.84355900 & -0.48318100 & 3. 38984500 \\
\hline $\mathrm{H}$ & -3.18006900 & -1.02844900 & 3. 77173800 \\
\hline H & -4.54980000 & -1.70276300 & 4. 65908100 \\
\hline $\mathrm{H}$ & -3.47136900 & -4.46796300 & 2. 29269100 \\
\hline H & -3.69471200 & -4.04676400 & 4. 01034800 \\
\hline H & -2.36601100 & -3.30764000 & 3. 10251700 \\
\hline $\mathrm{H}$ & -6.54377900 & -2.23623900 & 2. 42743900 \\
\hline H & -6.03651500 & -3.35398400 & 3. 71142100 \\
\hline $\mathrm{H}$ & -5.96878800 & -3.87593700 & 2. 01924200 \\
\hline H & -5.01961900 & -0.02327700 & -3.47189300 \\
\hline $\mathrm{H}$ & -5.88379200 & -1.27913900 & -4.38373200 \\
\hline $\mathrm{H}$ & -4.17530500 & -1.51297700 & -3.96496900 \\
\hline H & -4.76814800 & -3.75571700 & -2.86364600 \\
\hline H & -6.45283600 & -3.48782200 & -3.38094600 \\
\hline H & -6.09037100 & -3.85297100 & -1.67794400 \\
\hline $\mathrm{H}$ & -6.89099300 & -0.13421900 & -1.69608900 \\
\hline $\mathrm{H}$ & -7.37265100 & -1.69779200 & -0.99962500 \\
\hline $\mathrm{H}$ & -7.68568700 & -1.36083400 & -2.71475100 \\
\hline $\mathrm{H}$ & 0.29287700 & 2. 83635800 & -1.03024600 \\
\hline $\mathrm{H}$ & -3.01329900 & 5. 55683100 & -0.77341800 \\
\hline $\mathrm{H}$ & -3.65658500 & 1. 34562100 & -0.26150500 \\
\hline $\mathrm{H}$ & -5.16123200 & 2. 05495600 & 1. 25013800 \\
\hline H & -6.44272200 & 3. 27947000 & 1. 38967000 \\
\hline $\mathrm{H}$ & -4.79425300 & 3. 63680300 & 1. 97031200 \\
\hline $\mathrm{H}$ & -6.48026300 & 5. 29626600 & -0.03181500 \\
\hline $\mathrm{H}$ & -5.20589600 & 5. 65658300 & -1.21396400 \\
\hline $\mathrm{H}$ & -4.86472100 & 5. 76817400 & 0.53339000 \\
\hline $\mathrm{H}$ & -5.62627000 & 1. 89284700 & -1.31118900 \\
\hline $\mathrm{H}$ & -5.61448200 & 3. 37141300 & -2.29997900 \\
\hline $\mathrm{H}$ & -6.91824400 & 3. 09960700 & -1.11364600 \\
\hline $\mathrm{H}$ & 1. 21327500 & 4. 47252900 & -2.37174700 \\
\hline $\mathrm{H}$ & 1. 55881200 & 4. 60374800 & -0.62952500 \\
\hline $\mathrm{H}$ & 1. 73681700 & 6. 02371600 & -1.68089400 \\
\hline $\mathrm{H}$ & -1.37745700 & 6.85169300 & 0.20790700 \\
\hline $\mathrm{H}$ & 0.28357400 & 7. 35959300 & -0.17433800 \\
\hline $\mathrm{H}$ & -0.00199100 & 5. 96019100 & 0.89470000 \\
\hline $\mathrm{H}$ & -1.86889200 & 6.68695200 & -2.34570000 \\
\hline . & -0.82845300 & 5.67527400 & -3.37320800 \\
\hline
\end{tabular}




\begin{tabular}{|c|c|c|c|}
\hline $\mathrm{H}$ & -0.19700700 & 7. 18953400 & -2.67514600 \\
\hline $\mathrm{C}$ & 4. 64412400 & 0.06680200 & 0.81651700 \\
\hline $\mathrm{H}$ & 4. 77989700 & -0.92647200 & 1. 26710600 \\
\hline $\mathrm{H}$ & 4. 23760200 & 0.70886900 & 1. 61099400 \\
\hline C & 5. 95508900 & 0.64620100 & 0.32361100 \\
\hline $\mathrm{C}$ & 7. 09425800 & -0.17351900 & 0.00724000 \\
\hline $\mathrm{C}$ & 6. 05058300 & 2. 01959900 & 0.15538300 \\
\hline $\mathrm{C}$ & 7. 10712000 & -1.59327700 & 0.15580200 \\
\hline $\mathrm{C}$ & 8. 29385900 & 0.45840300 & -0.47991200 \\
\hline $\mathrm{C}$ & 7. 22853700 & 2. 63670000 & -0.32584900 \\
\hline $\mathrm{H}$ & 5. 19070400 & 2. 64643700 & 0.40972600 \\
\hline $\mathrm{C}$ & 8.22337500 & -2.34117200 & -0.15847000 \\
\hline $\mathrm{H}$ & 6.21989600 & -2.10757500 & 0.52732700 \\
\hline $\mathrm{C}$ & 9. 42736200 & -0.34432600 & -0.79358400 \\
\hline $\mathrm{C}$ & 8. 32921900 & 1. 87067300 & -0.63885900 \\
\hline $\mathrm{H}$ & 7. 25961100 & 3. 72295200 & -0.44156900 \\
\hline $\mathrm{C}$ & 9. 39804800 & -1.71302300 & -0.63934100 \\
\hline $\mathrm{H}$ & 8. 20151900 & -3.42649000 & -0.03191200 \\
\hline $\mathrm{H}$ & 10.33007500 & 0.15113700 & -1.16154700 \\
\hline $\mathrm{H}$ & 9. 24619200 & 2. 33660900 & -1.00944500 \\
\hline $\mathrm{H}$ & 10. 27673200 & -2.31500200 & -0.88378600 \\
\hline $\mathrm{C}$ & 1. 19953900 & 3. 39828900 & 2. 78899700 \\
\hline $\mathrm{H}$ & 0.63527700 & 4. 16289800 & 3. 35275900 \\
\hline $\mathrm{H}$ & 1. 25362500 & 3. 70666600 & 1. 73909800 \\
\hline $\mathrm{H}$ & 2. 21179700 & 3. 34398700 & 3. 21448100 \\
\hline $\mathrm{C}$ & 0.45607800 & 2. 08143800 & 2. 93098000 \\
\hline 0 & -0.54236600 & 1. 84924300 & 2. 16217800 \\
\hline $\mathrm{C}$ & 0.50442200 & 1. 45230100 & 4. 31973900 \\
\hline $\mathrm{H}$ & 1. 53691900 & 1. 13935600 & 4. 54255400 \\
\hline $\mathrm{H}$ & 0.28995900 & 2. 28091300 & 5. 02275700 \\
\hline C & -0.49324500 & 0.32716000 & 4. 56476600 \\
\hline $\mathrm{H}$ & -0.53107900 & 0.08203400 & 5. 63611800 \\
\hline $\mathrm{H}$ & -1.50248300 & 0.67802100 & 4. 28925000 \\
\hline $\mathrm{C}$ & -0.27734200 & -0.99753600 & 3. 81231100 \\
\hline 0 & -0.71691400 & -2.02514700 & 4. 33110900 \\
\hline 0 & 0.29559300 & -0.96706500 & 2. 65905200 \\
\hline $\mathrm{H}$ & 2. 21785200 & 0.90369700 & 2. 17943700 \\
\hline $\mathrm{H}$ & 1. 44378000 & 1. 76014200 & 0.32393600 \\
\hline
\end{tabular}

\section{TSDS}

Ir

$\mathrm{P}$

0

N
0. 89061400

$-0.91852000$

3. 15957900

2. 22138500
0. 64057000

0. 23828800

$-2.31059100$

$-0.60089900$
1. 00873300

$-0.53542000$

$-1.06378100$

0. 01695800 


\begin{tabular}{|c|c|c|c|}
\hline $\mathrm{C}$ & 3. 51787600 & -0.07398200 & -0.49626100 \\
\hline $\mathrm{C}$ & 3. 91022800 & -1.15865200 & -1.50707600 \\
\hline $\mathrm{C}$ & 2. 15191000 & -1.85269400 & -0.29534100 \\
\hline $\mathrm{C}$ & 1. 13427900 & -2.84659400 & 0.10209000 \\
\hline $\mathrm{C}$ & 1. 18491300 & -3.39431700 & 1. 39350700 \\
\hline $\mathrm{C}$ & 0.30930100 & -4.41625800 & 1. 76074900 \\
\hline $\mathrm{C}$ & -0.63438400 & -4.89608700 & 0.84895700 \\
\hline $\mathrm{C}$ & -0.71271700 & -4.32616500 & -0.42207900 \\
\hline $\mathrm{C}$ & -1.62856100 & -4.70624800 & -1.56137700 \\
\hline $\mathrm{C}$ & -1.46211500 & -3.53910200 & -2.55288400 \\
\hline $\mathrm{C}$ & -0.04665800 & -2.93097000 & -2.26700500 \\
\hline $\mathrm{C}$ & 0.16109700 & -3.29631100 & -0.80329700 \\
\hline $\mathrm{C}$ & 1. 01053600 & -3.67236700 & -3.14744400 \\
\hline $\mathrm{C}$ & 0.98226800 & -2.94288100 & -4.49233600 \\
\hline $\mathrm{C}$ & 0.58226000 & -1.53925100 & -4.10535600 \\
\hline $\mathrm{C}$ & 0.68270100 & -0.42451600 & -4.93530900 \\
\hline $\mathrm{C}$ & 0.22277700 & 0.80121000 & -4.46416400 \\
\hline $\mathrm{C}$ & -0.29948900 & 0.89264300 & -3.17417100 \\
\hline $\mathrm{C}$ & -0.39481500 & -0.21013300 & -2.29755400 \\
\hline $\mathrm{C}$ & 0.04628800 & -1.47488400 & -2.79464000 \\
\hline $\mathrm{C}$ & -2.34527000 & -0.85759000 & -0.11943700 \\
\hline $\mathrm{C}$ & -3.34318800 & -1.10600200 & -1.08350200 \\
\hline $\mathrm{C}$ & -4.51200000 & -1.79539700 & -0.74706600 \\
\hline $\mathrm{C}$ & -4.66405400 & -2.20189200 & 0.59466600 \\
\hline $\mathrm{C}$ & -3.70410200 & -1.95841700 & 1. 58384500 \\
\hline $\mathrm{C}$ & -2.52072400 & -1.29154000 & 1. 19752300 \\
\hline $\mathrm{C}$ & -3.92196900 & -2.36280600 & 3. 05756500 \\
\hline $\mathrm{C}$ & -3.82216200 & -1.10161300 & 3. 94706900 \\
\hline $\mathrm{C}$ & -2.84466600 & -3.38072500 & 3. 49451900 \\
\hline $\mathrm{C}$ & -5.30662200 & -2.99752300 & 3. 28650500 \\
\hline $\mathrm{C}$ & -5.62327800 & -2.11335700 & -1.77147100 \\
\hline $\mathrm{C}$ & -5.31612400 & -1.52892700 & -3.16245900 \\
\hline $\mathrm{C}$ & -5.76646100 & -3.64699100 & -1.91116900 \\
\hline $\mathrm{C}$ & -6.96856200 & -1.52335200 & -1.28876000 \\
\hline $\mathrm{C}$ & -1.77119200 & 1. 90790900 & -0.67118100 \\
\hline $\mathrm{C}$ & -0.99550100 & 3. 07026300 & -0.85916000 \\
\hline $\mathrm{C}$ & -1.57115300 & 4. 34165500 & -0.89387700 \\
\hline $\mathrm{C}$ & -2.96194600 & 4. 44070500 & -0.69458700 \\
\hline $\mathrm{C}$ & -3.76624400 & 3. 32031100 & -0.47601300 \\
\hline C & -3.14744900 & 2. 05330500 & -0.47572500 \\
\hline $\mathrm{C}$ & -5.28762600 & 3. 42152000 & -0.23999700 \\
\hline $\mathrm{C}$ & -5.64544500 & 2. 76631800 & 1. 11402100 \\
\hline $\mathrm{C}$ & -5.78076200 & 4. 87959600 & -0.21024100 \\
\hline $\mathrm{C}$ & -6.02804200 & 2. 68714100 & -1.38115300 \\
\hline $\mathrm{C}$ & -0.73669400 & 5. 62314400 & -1.09805400 \\
\hline
\end{tabular}




\begin{tabular}{|c|c|c|c|}
\hline $\mathrm{C}$ & 0.74040400 & 5. 31518300 & -1.40393100 \\
\hline $\mathrm{C}$ & -0.79799900 & 6. 47457900 & 0.19184200 \\
\hline $\mathrm{C}$ & -1.30712600 & 6. 43917600 & -2.28081000 \\
\hline $\mathrm{H}$ & 3. 33356700 & 0.88688500 & -0.99229400 \\
\hline $\mathrm{H}$ & 3.58145000 & -0.91591900 & -2.53033800 \\
\hline $\mathrm{H}$ & 4. 97560000 & -1.41727800 & -1.51392600 \\
\hline $\mathrm{H}$ & 1. 89658200 & -3.00791600 & 2. 12041100 \\
\hline H & 0.35382000 & -4.81000900 & 2. 77757300 \\
\hline $\mathrm{H}$ & -1.31643200 & -5.70200300 & 1. 13391800 \\
\hline $\mathrm{H}$ & -1.30946500 & -5.66753500 & -2.00403800 \\
\hline $\mathrm{H}$ & -2.67404800 & -4.83954900 & -1.24098200 \\
\hline $\mathrm{H}$ & -2.22644600 & -2.78204200 & -2.34950200 \\
\hline $\mathrm{H}$ & -1.56956800 & -3.84167800 & -3.60548200 \\
\hline $\mathrm{H}$ & 2. 00197200 & -3.57217200 & -2.68555400 \\
\hline $\mathrm{H}$ & 0.78763300 & -4.74737300 & -3.22068000 \\
\hline $\mathrm{H}$ & 1. 94517000 & -2.97648300 & -5.02726700 \\
\hline $\mathrm{H}$ & 0.22935100 & -3.37555400 & -5.17593100 \\
\hline $\mathrm{H}$ & 1. 10448300 & -0.52093300 & -5.93972300 \\
\hline $\mathrm{H}$ & 0.26854600 & 1. 69511000 & -5.09150700 \\
\hline $\mathrm{H}$ & -0.64664200 & 1. 86757600 & -2.84131000 \\
\hline $\mathrm{H}$ & -3.19820200 & -0.73235700 & -2.09674500 \\
\hline $\mathrm{H}$ & -5.57946300 & -2.72333800 & 0.86993600 \\
\hline $\mathrm{H}$ & -1.72322400 & -1.10704600 & 1. 91845200 \\
\hline $\mathrm{H}$ & -4.58265600 & -0.35447600 & 3. 66681000 \\
\hline $\mathrm{H}$ & -2.83397800 & -0.62727500 & 3. 87158400 \\
\hline $\mathrm{H}$ & -3.97854000 & -1.36932000 & 5. 00471700 \\
\hline $\mathrm{H}$ & -2.88310100 & -4.28292900 & 2. 86373400 \\
\hline $\mathrm{H}$ & -3.02352800 & -3.68893900 & 4. 53802300 \\
\hline $\mathrm{H}$ & -1.82629300 & -2.96807600 & 3. 45206700 \\
\hline $\mathrm{H}$ & -6.12825500 & -2.31547400 & 3. 01473500 \\
\hline $\mathrm{H}$ & -5.42399700 & -3.24647300 & 4. 35259900 \\
\hline $\mathrm{H}$ & -5.43274500 & -3.93230100 & 2. 71724600 \\
\hline $\mathrm{H}$ & -5.22499700 & -0.43186600 & -3.13660300 \\
\hline $\mathrm{H}$ & -6.13185500 & -1.77653100 & -3.85988400 \\
\hline $\mathrm{H}$ & -4.38584500 & -1.93774000 & -3.58627700 \\
\hline $\mathrm{H}$ & -4.83120700 & -4.10129500 & -2.27421100 \\
\hline $\mathrm{H}$ & -6.56492200 & -3.89570800 & -2.62975300 \\
\hline $\mathrm{H}$ & -6.02089000 & -4.12315700 & -0.95187600 \\
\hline $\mathrm{H}$ & -6.90544700 & -0.42990900 & -1.17690800 \\
\hline $\mathrm{H}$ & -7.27944700 & -1.94201000 & -0.31988200 \\
\hline $\mathrm{H}$ & -7.76680800 & -1.74424500 & -2.01638300 \\
\hline $\mathrm{H}$ & 0.08120300 & 2. 96401700 & -0.95748400 \\
\hline $\mathrm{H}$ & -3.41863200 & 5. 42953700 & -0.69898300 \\
\hline $\mathrm{H}$ & -3.75985800 & 1. 17177700 & -0.30139600 \\
\hline $\mathrm{H}$ & -5.36802200 & 1. 70213200 & 1. 14450000 \\
\hline
\end{tabular}




\begin{tabular}{|c|c|c|c|}
\hline H & -6.73041700 & 2. 83341200 & 1. 29870100 \\
\hline $\mathrm{H}$ & -5.12599800 & 3.27028600 & 1.94437100 \\
\hline H & -6.86748600 & 4. 90045000 & -0.03211800 \\
\hline $\mathrm{H}$ & -5.59515700 & 5. 39912500 & -1.16346900 \\
\hline $\mathrm{H}$ & -5.30321000 & 5. 45920600 & 0.59531700 \\
\hline $\mathrm{H}$ & -5.73514200 & 1. 62833100 & -1.43919800 \\
\hline $\mathrm{H}$ & -5.80747200 & 3. 14936000 & -2.35669000 \\
\hline H & -7.11883600 & 2. 72596600 & -1.22438200 \\
\hline $\mathrm{H}$ & 0.85243000 & 4. 70594800 & -2.31467000 \\
\hline H & 1. 23029900 & 4. 78071300 & -0.57610800 \\
\hline H & 1. 29250800 & 6.25499000 & -1.56279300 \\
\hline $\mathrm{H}$ & -1.83033600 & 6. 76553500 & 0.43973900 \\
\hline H & -0.20823100 & 7. 39913500 & 0.07486100 \\
\hline $\mathrm{H}$ & -0.39404000 & 5. 91459600 & 1. 04990600 \\
\hline H & -2.35176400 & 6.74086300 & -2.11184100 \\
\hline $\mathrm{H}$ & -1.27370100 & 5. 85731100 & -3.21596900 \\
\hline $\mathrm{H}$ & -0.71844600 & 7. 35904500 & -2.43181200 \\
\hline $\mathrm{C}$ & 4. 52482600 & 0.13292400 & 0.65663700 \\
\hline $\mathrm{H}$ & 4. 65265600 & -0.81716900 & 1. 19415900 \\
\hline $\mathrm{H}$ & 4. 05752500 & 0.82600600 & 1. 37095000 \\
\hline $\mathrm{C}$ & 5.85024400 & 0.70713900 & 0.19720800 \\
\hline $\mathrm{C}$ & 7. 02953200 & -0.09991300 & 0.03160800 \\
\hline $\mathrm{C}$ & 5. 91958500 & 2. 06189700 & -0.09211100 \\
\hline $\mathrm{C}$ & 7. 07075800 & -1.49898500 & 0.31194400 \\
\hline $\mathrm{C}$ & 8. 24127300 & 0.52434200 & -0.43518100 \\
\hline $\mathrm{C}$ & 7. 10953300 & 2. 67117500 & -0.55314100 \\
\hline $\mathrm{H}$ & 5. 02779600 & 2. 68037000 & 0.04666700 \\
\hline $\mathrm{C}$ & 8. 22536900 & -2.23493900 & 0.14087800 \\
\hline $\mathrm{H}$ & 6. 17510800 & -2.00630200 & 0.67250000 \\
\hline $\mathrm{C}$ & 9. 41445800 & -0.26563600 & -0.59969900 \\
\hline $\mathrm{C}$ & 8.24888000 & 1. 91656700 & -0.72265000 \\
\hline $\mathrm{H}$ & 7. 11875500 & 3. 74264900 & -0.76804900 \\
\hline $\mathrm{C}$ & 9.41202600 & -1.61470600 & -0.32047000 \\
\hline $\mathrm{H}$ & 8. 22449200 & -3.30422100 & 0.36675800 \\
\hline $\mathrm{H}$ & 10.32587000 & 0.22387600 & -0.95392900 \\
\hline $\mathrm{H}$ & 9. 17543900 & 2.37678500 & -1.07618300 \\
\hline $\mathrm{H}$ & 10.32106300 & -2.20701300 & -0.45090500 \\
\hline $\mathrm{C}$ & 1. 80306300 & 3. 42831700 & 2. 59781300 \\
\hline $\mathrm{H}$ & 1. 29357600 & 4. 22005300 & 3. 17300100 \\
\hline $\mathrm{H}$ & 1. 81082700 & 3. 71754400 & 1. 54015600 \\
\hline $\mathrm{H}$ & 2. 83564300 & 3. 34510300 & 2. 96899800 \\
\hline $\mathrm{C}$ & 1. 01081500 & 2. 13889200 & 2. 79533300 \\
\hline 0 & -0.13892200 & 2. 05600100 & 2. 18845200 \\
\hline $\mathrm{C}$ & 1. 11841000 & 1. 53663800 & 4. 20109000 \\
\hline $\mathrm{H}$ & 2. 15552400 & 1. 21617400 & 4. 39418200 \\
\hline
\end{tabular}




$\begin{array}{rrrr}\mathrm{H} & 0.93551800 & 2.38622200 & 4.88638500 \\ \mathrm{C} & 0.11594500 & 0.42977800 & 4.52316600 \\ \mathrm{H} & 0.13597800 & 0.21694800 & 5.60115800 \\ \mathrm{H} & -0.89342900 & 0.79844900 & 4.27523600 \\ \mathrm{C} & 0.30041100 & -0.91232800 & 3.79541600 \\ 0 & 0.23697600 & -1.95296900 & 4.44815100 \\ \mathrm{O} & 0.45091800 & -0.88658100 & 2.51153700 \\ \mathrm{H} & 2.05929200 & 1.20902800 & 2.06165900 \\ \mathrm{H} & 1.32969000 & 1.75266400 & -0.02753800\end{array}$

\section{IntES}

Ir

$\mathrm{P}$

0

$\mathrm{N}$

C

C

C

C

C

C

C

C

C

C

C

C

C

C

C

C

C

C

C

C

C

C

C

C

C

C

C

C

C
0. 83164200

$-0.95145200$

2. 37023800

1. 89102000

3. 11249000

3. 27113200

1. 61347700

0. 53746500

0. 80086100

$-0.19653600$

$-1.46501500$

$-1.73077800$

$-2.99386900$

$-2.77733500$

$-1.22823500$

$-0.74269100$

$-0.65033000$

$-0.79781100$

$-0.69243200$

$-0.46830500$

$-0.44414100$

$-0.63321400$

$-0.84901800$

$-0.88205900$

$-2.65855400$

$-3.73298100$

$-5.04231600$

$-5.24514000$

$-4.20387600$

$-2.89706200$

$-4.43420700$

$-4.00520600$

$-3.57897400$
$-0.34786900$

0. 23364400

$-3.17468100$

$-1.37175500$

$-0.97312200$

$-2.15363500$

$-2.58951400$

$-3.35623800$

$-3.93850000$

$-4.65339100$

$-4.79461800$

$-4.20240700$

$-4.28416100$

$-3.22493500$

$-3.02771400$

$-3.46641400$

$-4.01609300$

$-3.29065100$

$-1.83821100$

$-0.78505500$

0. 51314100

0. 73258100

$-0.31029600$

$-1.64345600$

$-0.24315400$

$-0.15388400$

$-0.43253200$

$-0.80189600$

$-0.88063300$

$-0.59067500$

$-1.24000900$

$-0.04089600$

-2. 47322000
0. 85002300

$-0.50169800$

$-1.87556100$

$-0.64588900$

$-1.39004200$

$-2.38153900$

$-0.93857600$

$-0.27038200$

0. 98240000

1. 65005100

1. 07775300

$-0.15817100$

$-0.98167600$

$-2.08123600$

$-2.20602400$

$-0.83097500$

$-3.27132800$

$-4.60932600$

$-4.21322400$

$-5.09925800$

$-4.60140900$

$-3.23575900$

$-2.30950900$

$-2.82371100$

0. 02399000

$-0.88335600$

$-0.47847900$

0. 86797900

1. 79931700

1. 35510200

3. 28094600

4. 15826100

3. 65173500 


\begin{tabular}{|c|c|c|c|}
\hline $\mathrm{C}$ & -5.90742900 & -1.56453700 & 3. 58583500 \\
\hline $\mathrm{C}$ & -6.24756000 & -0.35434900 & -1.43960400 \\
\hline $\mathrm{C}$ & -5.83539700 & 0.09082200 & -2.85438000 \\
\hline $\mathrm{C}$ & -6.91076900 & -1.74762800 & -1.54594000 \\
\hline $\mathrm{C}$ & -7.27833700 & 0.66208900 & -0.89618400 \\
\hline $\mathrm{C}$ & -1.07277000 & 2. 09741100 & -0.56668700 \\
\hline $\mathrm{C}$ & 0.09487900 & 2. 85232200 & -0.79759000 \\
\hline $\mathrm{C}$ & 0.07974700 & 4. 24870600 & -0.78100000 \\
\hline $\mathrm{C}$ & -1.14698300 & 4. 88815000 & -0.50802800 \\
\hline $\mathrm{C}$ & -2.32485200 & 4. 17897000 & -0.25944300 \\
\hline $\mathrm{C}$ & -2.26560100 & 2. 77088500 & -0.29803400 \\
\hline $\mathrm{C}$ & -3.66636600 & 4. 87074800 & 0.05914700 \\
\hline $\mathrm{C}$ & -4.16514800 & 4. 40721900 & 1. 44766400 \\
\hline $\mathrm{C}$ & -3.54325500 & 6. 40501300 & 0.08104400 \\
\hline $\mathrm{C}$ & -4.70861100 & 4. 48688900 & -1.01663700 \\
\hline $\mathrm{C}$ & 1. 34450500 & 5.09716600 & -1.02723800 \\
\hline $\mathrm{C}$ & 2.58566900 & 4. 22976700 & -1.30482100 \\
\hline $\mathrm{C}$ & 1. 63189300 & 5.96042400 & 0.22350700 \\
\hline $\mathrm{C}$ & 1. 11648700 & 6. 01913700 & -2.24752400 \\
\hline $\mathrm{H}$ & 2. 90347100 & -0.03106700 & -1.91906400 \\
\hline $\mathrm{H}$ & 2. 95275900 & -1.90737800 & -3.40622600 \\
\hline $\mathrm{H}$ & 4. 28411600 & -2.57619800 & -2.40146700 \\
\hline $\mathrm{H}$ & 1. 78946600 & -3.82046500 & 1. 43062800 \\
\hline $\mathrm{H}$ & 0.02140000 & -5.10167300 & 2. 62211300 \\
\hline $\mathrm{H}$ & -2.24103500 & -5.36553400 & 1. 59463700 \\
\hline $\mathrm{H}$ & -3.10079400 & -5.30006000 & -1.40319400 \\
\hline $\mathrm{H}$ & -3.90213400 & -4.09191900 & -0.38875600 \\
\hline $\mathrm{H}$ & -3.23691700 & -2.28255100 & -1.76703600 \\
\hline $\mathrm{H}$ & -3.22844800 & -3.50410200 & -3.04535900 \\
\hline $\mathrm{H}$ & 0.41529500 & -4.18901200 & -3.06230800 \\
\hline $\mathrm{H}$ & -1.15780700 & -4.99176500 & -3.23289600 \\
\hline $\mathrm{H}$ & -0.03671400 & -3.58577200 & -5.34922900 \\
\hline $\mathrm{H}$ & -1.78082900 & -3.48499900 & -5.07534000 \\
\hline $\mathrm{H}$ & -0.32337100 & -0.98332300 & -6.16485900 \\
\hline $\mathrm{H}$ & -0.28009300 & 1. 36472800 & -5.26656000 \\
\hline $\mathrm{H}$ & -0.61346700 & 1. 76169400 & -2.88346200 \\
\hline $\mathrm{H}$ & -3.52846300 & 0.14019100 & -1.91242100 \\
\hline $\mathrm{H}$ & -6.26088500 & -1.02380700 & 1. 19347300 \\
\hline $\mathrm{H}$ & -2.05696800 & -0.58390600 & 2. 05342200 \\
\hline $\mathrm{H}$ & -4.61077700 & 0.85123300 & 3. 93093500 \\
\hline $\mathrm{H}$ & -2.94892300 & 0.22033700 & 3. 99902900 \\
\hline $\mathrm{H}$ & -4.13935900 & -0.27919000 & 5. 22633600 \\
\hline $\mathrm{H}$ & -3.86077600 & -3.34788300 & 3. 04358400 \\
\hline $\mathrm{H}$ & -3.72550400 & -2.73509600 & 4. 71247900 \\
\hline $\mathrm{H}$ & -2.50582000 & -2.28797100 & 3. 49843300 \\
\hline
\end{tabular}




\begin{tabular}{|c|c|c|c|}
\hline H & -6.57011800 & -0.70834000 & 3. 38361300 \\
\hline $\mathrm{H}$ & -6.01887400 & -1.81935500 & 4. 65144800 \\
\hline $\mathrm{H}$ & -6.27077400 & -2.42522600 & 3. 00171000 \\
\hline $\mathrm{H}$ & -5.37428300 & 1.09079200 & -2.85335700 \\
\hline H & -6.72346800 & 0.13795300 & -3.50406600 \\
\hline $\mathrm{H}$ & -5.12510200 & -0.61156900 & -3.31766100 \\
\hline $\mathrm{H}$ & -6.20231500 & -2.49384000 & -1.93986400 \\
\hline $\mathrm{H}$ & -7.77752600 & -1.71117600 & -2.22623100 \\
\hline $\mathrm{H}$ & -7.26937400 & -2.10791500 & -0.56968300 \\
\hline H & -6.83682600 & 1. 66797800 & -0.81461300 \\
\hline $\mathrm{H}$ & -7.65247700 & 0.37899500 & 0.09928300 \\
\hline H & -8.14726900 & 0.72704100 & -1.57155100 \\
\hline H & 1. 02887300 & 2. 32839200 & -0.98952700 \\
\hline $\mathrm{H}$ & -1.16731000 & 5. 97676900 & -0.48187200 \\
\hline H & -3.16895700 & 2. 19768600 & -0.09878700 \\
\hline $\mathrm{H}$ & -4.32441600 & 3. 31913600 & 1. 48711800 \\
\hline H & -5.12325500 & 4. 89450000 & 1. 69297000 \\
\hline $\mathrm{H}$ & -3.43899300 & 4. 66661200 & 2. 23413000 \\
\hline $\mathrm{H}$ & -4.52195600 & 6.85261800 & 0.31512500 \\
\hline $\mathrm{H}$ & -3.22183200 & 6. 80770800 & -0.89240100 \\
\hline $\mathrm{H}$ & -2.83168100 & 6. 75009200 & 0.84735700 \\
\hline H & -4.87369200 & 3. 39942000 & -1.05622700 \\
\hline $\mathrm{H}$ & -4.38334300 & 4. 81375500 & -2.01729800 \\
\hline $\mathrm{H}$ & -5.67880900 & 4. 96458000 & -0.80148100 \\
\hline $\mathrm{H}$ & 2. 46122200 & 3. 60789700 & -2.20551500 \\
\hline $\mathrm{H}$ & 2. 82423000 & 3.56642100 & -0.45928100 \\
\hline $\mathrm{H}$ & 3. 46164900 & 4. 87591900 & -1.47150900 \\
\hline $\mathrm{H}$ & 0.80073900 & 6.64486300 & 0.45206700 \\
\hline $\mathrm{H}$ & 2. 53468800 & 6. 57347900 & 0.06748800 \\
\hline $\mathrm{H}$ & 1. 79933000 & 5. 32763500 & 1. 10921000 \\
\hline $\mathrm{H}$ & 0.26385900 & 6. 69912900 & -2.09806800 \\
\hline $\mathrm{H}$ & 0.91952300 & 5. 42979200 & -3.15758400 \\
\hline $\mathrm{H}$ & 2. 00861600 & 6. 63989700 & -2.43202300 \\
\hline $\mathrm{C}$ & 4. 27977200 & -0.79277900 & -0.41662900 \\
\hline $\mathrm{H}$ & 4. 48275700 & -1.77175700 & 0.05289200 \\
\hline $\mathrm{H}$ & 3.92492900 & -0.17964900 & 0.42329400 \\
\hline $\mathrm{C}$ & 5.56760800 & -0.22702600 & -0.96845500 \\
\hline $\mathrm{C}$ & 6.65820500 & -0.01431900 & -0.04922100 \\
\hline $\mathrm{C}$ & 5. 74718400 & 0.08886500 & -2.30441900 \\
\hline $\mathrm{C}$ & 6.54723200 & -0.28523900 & 1. 34752500 \\
\hline $\mathrm{C}$ & 7. 90462100 & 0.49740600 & -0.54913600 \\
\hline $\mathrm{C}$ & 6. 97692300 & 0.60174600 & -2.79250500 \\
\hline $\mathrm{H}$ & 4. 93279500 & -0.04969200 & -3.01975500 \\
\hline $\mathrm{C}$ & 7. 61691800 & -0.07009700 & 2. 19496100 \\
\hline 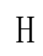 & 5. 61410600 & -0.65207700 & 1. 78252900 \\
\hline
\end{tabular}




$\begin{array}{rrrr}\text { C } & 8.98428700 & 0.70191600 & 0.35437700 \\ \mathrm{C} & 8.03445000 & 0.79618300 & -1.93593700 \\ \mathrm{H} & 7.07557600 & 0.83760500 & -3.85535600 \\ \mathrm{C} & 8.84698200 & 0.42295900 & 1.69802400 \\ \mathrm{H} & 7.50203200 & -0.28090200 & 3.26101500 \\ \mathrm{H} & 9.92879200 & 1.08939700 & -0.03826700 \\ \mathrm{H} & 8.98722300 & 1.18587200 & -2.30497200 \\ \mathrm{H} & 9.68440000 & 0.58642500 & 2.38159300 \\ \mathrm{C} & -0.27864400 & 2.19213900 & 3.99257600 \\ \mathrm{H} & -1.26460800 & 1.86775400 & 4.36427600 \\ \mathrm{H} & -0.44388600 & 2.91419200 & 3.17818300 \\ \mathrm{H} & 0.24469600 & 2.70727700 & 4.81562700 \\ \mathrm{C} & 0.52587300 & 0.99313300 & 3.48498800 \\ \mathrm{O} & -0.17976500 & 0.37458800 & 2.42955300 \\ \mathrm{C} & 0.82886700 & -0.00115100 & 4.62009800 \\ \mathrm{H} & 1.49740300 & 0.48899600 & 5.34980700 \\ \mathrm{H} & -0.10912700 & -0.23499100 & 5.15603300 \\ \mathrm{C} & 1.46567200 & -1.32005400 & 4.15096400 \\ \mathrm{H} & 1.77213900 & -1.91304900 & 5.02517500 \\ \mathrm{H} & 0.71507900 & -1.90184200 & 3.59072500 \\ \mathrm{C} & 2.70179700 & -1.11893300 & 3.27176000 \\ \mathrm{O} & 3.82951000 & -1.25510600 & 3.72518200 \\ \mathrm{O} & 2.49829700 & -0.80043500 & 2.01674900 \\ \mathrm{H} & 1.49581200 & 1.37837900 & 3.11465500 \\ \mathrm{H} & 1.54519600 & 0.90432600 & 0.32056200\end{array}$

\section{IntFR}

$\begin{array}{rrrr}\text { Ir } & 0.72035500 & -0.04550100 & 1.07614400 \\ \mathrm{P} & -0.94690900 & 0.02763400 & -0.50614000 \\ \mathrm{O} & 2.18822800 & -3.39549700 & -0.94924800 \\ \mathrm{~N} & 1.79979700 & -1.46607100 & 0.08399800 \\ \mathrm{C} & 3.01273900 & -1.19555000 & -0.75233700 \\ \mathrm{C} & 3.03445900 & -2.44644200 & -1.65549600 \\ \mathrm{C} & 1.47319200 & -2.71091900 & -0.06586600 \\ \mathrm{C} & 0.37010900 & -3.40018100 & 0.63431100 \\ \mathrm{C} & 0.51641400 & -3.75503900 & 1.98667000 \\ \mathrm{C} & -0.52835400 & -4.38797700 & 2.66266300 \\ \mathrm{C} & -1.73471800 & -4.65538700 & 2.00385900 \\ \mathrm{C} & -1.88547300 & -4.28567200 & 0.66638800 \\ \mathrm{C} & -3.06799700 & -4.51445300 & -0.24520300 \\ \mathrm{C} & -2.75454400 & -3.62541300 & -1.46591000 \\ \mathrm{C} & -1.19454100 & -3.49379800 & -1.50071000 \\ \mathrm{C} & -0.83782600 & -3.65726700 & -0.02800800 \\ \mathrm{C} & -0.58776600 & -4.70230800 & -2.29102200\end{array}$




\begin{tabular}{|c|c|c|c|}
\hline $\mathrm{C}$ & -0.58749600 & -4.25887900 & -3.75468700 \\
\hline $\mathrm{C}$ & -0.42314700 & -2.76353200 & -3.64839100 \\
\hline $\mathrm{C}$ & -0.04415700 & -1.92110700 & -4.69346300 \\
\hline $\mathrm{C}$ & 0.03671100 & -0.55146700 & -4.46035000 \\
\hline $\mathrm{C}$ & -0.25808000 & -0.04925900 & -3.19222000 \\
\hline $\mathrm{C}$ & -0.64031700 & -0.87915100 & -2.11497400 \\
\hline $\mathrm{C}$ & -0.72495900 & -2.28399800 & -2.35089400 \\
\hline $\mathrm{C}$ & -2.70130400 & -0.31192000 & -0.07799100 \\
\hline $\mathrm{C}$ & -3.67055000 & -0.18652100 & -1.08931200 \\
\hline $\mathrm{C}$ & -5.03093000 & -0.37822600 & -0.81146500 \\
\hline $\mathrm{C}$ & -5.37971000 & -0.70561100 & 0.51083700 \\
\hline $\mathrm{C}$ & -4.44159900 & -0.85195700 & 1. 54436400 \\
\hline $\mathrm{C}$ & -3.08801600 & -0.64441500 & 1. 22622900 \\
\hline $\mathrm{C}$ & -4.92015600 & -1.22534900 & 2. 96304100 \\
\hline $\mathrm{C}$ & -3.74777800 & -1.40398100 & 3. 94486100 \\
\hline $\mathrm{C}$ & -5.70679600 & -2.55575100 & 2. 91121500 \\
\hline $\mathrm{C}$ & -5.84139900 & -0.10513800 & 3. 50019800 \\
\hline $\mathrm{C}$ & -6.12880500 & -0.25365000 & -1.88781700 \\
\hline $\mathrm{C}$ & -5.54942100 & 0.08900800 & -3.27228500 \\
\hline $\mathrm{C}$ & -6.88915600 & -1.59566700 & -2.00075700 \\
\hline $\mathrm{C}$ & -7.11652400 & 0.86543700 & -1.48282900 \\
\hline $\mathrm{C}$ & -0.90051200 & 1.84793600 & -0.88485400 \\
\hline $\mathrm{C}$ & 0.36751800 & 2. 44451300 & -1.07336600 \\
\hline $\mathrm{C}$ & 0.50740300 & 3. 82701800 & -1.23135700 \\
\hline $\mathrm{C}$ & -0.66700200 & 4. 60769400 & -1.18463100 \\
\hline $\mathrm{C}$ & -1.93906800 & 4. 05953700 & -0.99024700 \\
\hline $\mathrm{C}$ & -2.03658300 & 2. 66025500 & -0.83675700 \\
\hline $\mathrm{C}$ & -3.21844500 & 4. 91934000 & -0.93443800 \\
\hline $\mathrm{C}$ & -3.91815000 & 4. 71053100 & 0.42880500 \\
\hline $\mathrm{C}$ & -2.92167300 & 6.42098600 & -1.09764000 \\
\hline $\mathrm{C}$ & -4.17024800 & 4. 48782500 & -2.07441200 \\
\hline $\mathrm{C}$ & 1. 87459500 & 4. 50649600 & -1.44466900 \\
\hline $\mathrm{C}$ & 3. 02978400 & 3. 48890300 & -1.47465400 \\
\hline $\mathrm{C}$ & 2. 13915800 & 5. 49259500 & -0.28175500 \\
\hline $\mathrm{C}$ & 1. 85415300 & 5. 27271500 & -2.78765500 \\
\hline $\mathrm{H}$ & -0.26256200 & -1.10247400 & 1. 63073700 \\
\hline $\mathrm{H}$ & 2. 82385700 & -0.28222100 & -1.33288200 \\
\hline $\mathrm{H}$ & 2. 58638600 & -2.28389800 & -2.64743900 \\
\hline $\mathrm{H}$ & 4. 02988500 & -2.89308400 & -1.76192400 \\
\hline $\mathrm{H}$ & 1. 45336600 & -3.53639400 & 2. 50334900 \\
\hline $\mathrm{H}$ & -0.39999000 & -4.67404800 & 3. 70903500 \\
\hline $\mathrm{H}$ & -2.54933300 & -5.15644800 & 2.53355100 \\
\hline $\mathrm{H}$ & -3.12733400 & -5.58233500 & -0.52107300 \\
\hline $\mathrm{H}$ & -4.02923100 & -4.25994900 & 0.22724400 \\
\hline $\mathrm{H}$ & -3.20407800 & -2.63633400 & -1.32300000 \\
\hline
\end{tabular}




\begin{tabular}{|c|c|c|c|}
\hline H & -3.14891200 & -4.03153400 & -2.40899200 \\
\hline $\mathrm{H}$ & 0.44554900 & -4.86997000 & -1.95607400 \\
\hline $\mathrm{H}$ & -1.15037300 & -5.62898100 & -2.10696200 \\
\hline $\mathrm{H}$ & 0.20647100 & -4.73289500 & -4.35238000 \\
\hline $\mathrm{H}$ & -1.54274400 & -4.49755300 & -4.25573900 \\
\hline $\mathrm{H}$ & 0.17940200 & -2.33601000 & -5.67980700 \\
\hline H & 0.32375900 & 0.13581000 & -5.25960300 \\
\hline $\mathrm{H}$ & -0.19583300 & 1. 02725300 & -3.04593700 \\
\hline $\mathrm{H}$ & -3.35318500 & 0.05512500 & -2.10321300 \\
\hline H & -6.43473400 & -0.85882000 & 0.74361800 \\
\hline $\mathrm{H}$ & -2.32384000 & -0.75010500 & 1. 99406900 \\
\hline H & -3.17099600 & -0.47486300 & 4. 07207100 \\
\hline $\mathrm{H}$ & -3.05600600 & -2.19742200 & 3. 62186600 \\
\hline $\mathrm{H}$ & -4.13395700 & -1.68443600 & 4. 93675900 \\
\hline H & -6.59552800 & -2.48946700 & 2. 26598700 \\
\hline $\mathrm{H}$ & -6.05236500 & -2.83419200 & 3. 91945800 \\
\hline H & -5.07488900 & -3.37464900 & 2. 53211700 \\
\hline H & -5.30756700 & 0.85710200 & 3. 55495000 \\
\hline $\mathrm{H}$ & -6.19716500 & -0.35351800 & 4. 51293000 \\
\hline $\mathrm{H}$ & -6.72745400 & 0.04003500 & 2. 86389400 \\
\hline $\mathrm{H}$ & -5.02347400 & 1. 05668000 & -3.27443000 \\
\hline $\mathrm{H}$ & -6.36486100 & 0.15972900 & -4.00804600 \\
\hline $\mathrm{H}$ & -4.85082000 & -0.68328000 & -3.63087800 \\
\hline $\mathrm{H}$ & -6.21016200 & -2.41396700 & -2.28914600 \\
\hline $\mathrm{H}$ & -7.67688400 & -1.52429100 & -2.76743300 \\
\hline $\mathrm{H}$ & -7.37488600 & -1.87826300 & -1.05450200 \\
\hline $\mathrm{H}$ & -6.60402600 & 1. 83713200 & -1.39992500 \\
\hline $\mathrm{H}$ & -7.60380400 & 0.65905200 & -0.51773900 \\
\hline $\mathrm{H}$ & -7.91106300 & 0.96648200 & -2.23928900 \\
\hline $\mathrm{H}$ & 1. 25855100 & 1. 81721200 & -1.09930200 \\
\hline $\mathrm{H}$ & -0.56947800 & 5.68585700 & -1.30053000 \\
\hline $\mathrm{H}$ & -3.01317600 & 2.21004500 & -0.66596400 \\
\hline $\mathrm{H}$ & -4.20512000 & 3. 66036400 & 0.59206800 \\
\hline $\mathrm{H}$ & -4.83620900 & 5.31697000 & 0.48392900 \\
\hline $\mathrm{H}$ & -3.26296100 & 5. 01690400 & 1. 26035000 \\
\hline $\mathrm{H}$ & -3.86217700 & 6.99075500 & -1.04949300 \\
\hline $\mathrm{H}$ & -2.45190800 & 6.64667200 & -2.06742700 \\
\hline $\mathrm{H}$ & -2.26593800 & 6.80263900 & -0.29954100 \\
\hline $\mathrm{H}$ & -4.46178800 & 3. 42959100 & -1.98939700 \\
\hline $\mathrm{H}$ & -3.69828900 & 4. 62923000 & -3.05953600 \\
\hline $\mathrm{H}$ & -5.09282200 & 5. 08963000 & -2.05147500 \\
\hline $\mathrm{H}$ & 2.91974100 & 2. 76257700 & -2.29707200 \\
\hline $\mathrm{H}$ & 3. 11680100 & 2. 94955600 & -0.51951600 \\
\hline $\mathrm{H}$ & 3.98093000 & 4. 01807500 & -1.63798300 \\
\hline . & 1. 35804300 & 6.26529100 & -0.20722000 \\
\hline
\end{tabular}




$\begin{array}{rrrr}\mathrm{H} & 3.09901500 & 6.00981900 & -0.43988600 \\ \mathrm{H} & 2.20123300 & 4.95631300 & 0.67707800 \\ \mathrm{H} & 1.08213300 & 6.05710800 & -2.80885000 \\ \mathrm{H} & 1.66536100 & 4.59173500 & -3.63337000 \\ \mathrm{H} & 2.82603500 & 5.76228700 & -2.95815900 \\ \mathrm{C} & 4.27667200 & -1.04389300 & 0.09980300 \\ \mathrm{H} & 4.42385100 & -1.99214900 & 0.64714700 \\ \mathrm{H} & 4.08153800 & -0.27876400 & 0.86558000 \\ \mathrm{C} & 5.54110400 & -0.70274000 & -0.66856700 \\ \mathrm{C} & 6.80068700 & -0.71669100 & 0.03056800 \\ \mathrm{C} & 5.53151700 & -0.36729500 & -2.01259900 \\ \mathrm{C} & 6.90993100 & -1.02365100 & 1.41763200 \\ \mathrm{C} & 8.00575300 & -0.40245200 & -0.68684300 \\ \mathrm{C} & 6.72127500 & -0.05048900 & -2.71641400 \\ \mathrm{H} & 4.59343300 & -0.33314600 & -2.57090200 \\ \mathrm{C} & 8.13436200 & -1.03226700 & 2.05565100 \\ \mathrm{H} & 6.01356300 & -1.24941600 & 1.99665200 \\ \mathrm{C} & 9.25126800 & -0.42319200 & 0.00033800 \\ \mathrm{C} & 7.93363900 & -0.07248200 & -2.06968400 \\ \mathrm{H} & 6.66400700 & 0.20840400 & -3.77637600 \\ \mathrm{C} & 9.31907000 & -0.73251400 & 1.34200100 \\ \mathrm{H} & 8.19018000 & -1.27013300 & 3.12085400 \\ \mathrm{H} & -0.15610100 & 2.41576600 & 1.76885000 \\ \mathrm{H} & & & \\ \mathrm{H} & & & \\ \mathrm{H} & & & \end{array}$




\section{IntGR1}

\begin{tabular}{|c|c|c|c|}
\hline Ir & 0.48165300 & 0.30011600 & 1. 14685400 \\
\hline $\mathrm{P}$ & -1.27678200 & 0.10216800 & -0.40269100 \\
\hline 0 & 2. 08990900 & -3.09512100 & -0.76041300 \\
\hline $\mathrm{N}$ & 1. 66845500 & -1.13794700 & 0.20089400 \\
\hline C & 2. 86677000 & -0.89506800 & -0.66130300 \\
\hline C & 2. 80036900 & -2.11979600 & -1.58094200 \\
\hline C & 1. 39547500 & -2.40520800 & 0.13629000 \\
\hline C & 0.40090600 & -3.11148800 & 0.96763200 \\
\hline $\mathrm{C}$ & 0.66338900 & -3.30594800 & 2. 33601600 \\
\hline C & -0.27820800 & -3.94079100 & 3. 14724800 \\
\hline C & -1.49945600 & -4.36604800 & 2. 60985000 \\
\hline C & -1.76773200 & -4.15335500 & 1. 25683200 \\
\hline C & -2.98596300 & -4.56405900 & 0.46401800 \\
\hline C & -2.82108600 & -3.80149700 & -0.86682300 \\
\hline C & -1.28441000 & -3.54586800 & -1.02266800 \\
\hline C & -0.82227100 & -3.52785400 & 0.42686500 \\
\hline C & -0.62452100 & -4.77512700 & -1.73327200 \\
\hline C & -0.76074200 & -4.48540400 & -3.22804900 \\
\hline C & -0.72033800 & -2.97860900 & -3.27819000 \\
\hline C & -0.48298300 & -2.22456100 & -4.42689100 \\
\hline C & -0.50589300 & -0.83671000 & -4.33640800 \\
\hline C & -0.75997000 & -0.23043300 & -3.10603000 \\
\hline C & -0.99639400 & -0.96657300 & -1.92337200 \\
\hline C & -0.97625400 & -2.39258900 & -2.01413900 \\
\hline C & -2.93161900 & -0.36463000 & 0.24796300 \\
\hline C & -3.99060000 & -0.50730100 & -0.66672300 \\
\hline C & -5.29283600 & -0.78135200 & -0.22564700 \\
\hline C & -5.49493300 & -0.89721000 & 1. 16124200 \\
\hline C & -4.46504300 & -0.76342600 & 2. 10457200 \\
\hline C & -3.17290600 & -0.49814800 & 1. 62068100 \\
\hline C & -4.77441100 & -0.90895600 & 3. 60871500 \\
\hline C & -3.51972500 & -0.72495700 & 4. 48173100 \\
\hline C & -5.34970100 & -2.31880100 & 3. 87902800 \\
\hline $\mathrm{C}$ & -5.81289700 & 0.15960100 & 4. 02300600 \\
\hline C & -6.47923200 & -0.97028900 & -1.19395200 \\
\hline C & -6.06276900 & -0.79123800 & -2.66504400 \\
\hline C & -7.05063400 & -2.39755000 & -1.02263200 \\
\hline C & -7.58025700 & 0.06622700 & -0.87084100 \\
\hline C & -1.50349900 & 1. 84384100 & -1.00601300 \\
\hline C & -0.36411500 & 2. 55306400 & -1.44289700 \\
\hline C & -0.45448000 & 3.88785600 & -1.84774300 \\
\hline C & -1.71824800 & 4. 50854300 & -1.77721600 \\
\hline C & -2.86627300 & 3. 84768500 & -1.32962600 \\
\hline C & -2.73836700 & 2. 49725800 & -0.94674800 \\
\hline
\end{tabular}




\begin{tabular}{|c|c|c|c|}
\hline $\mathrm{C}$ & -4.24430200 & 4. 53553000 & -1.24208200 \\
\hline $\mathrm{C}$ & -4.74196700 & 4. 49765700 & 0.22173100 \\
\hline $\mathrm{C}$ & -4.19281000 & 6. 00536100 & -1.69614300 \\
\hline $\mathrm{C}$ & -5.24810200 & 3. 78570800 & -2.14825900 \\
\hline $\mathrm{C}$ & 0.76889400 & 4. 69252000 & -2.33119500 \\
\hline $\mathrm{C}$ & 2. 02267500 & 3. 81056100 & -2.47623100 \\
\hline $\mathrm{C}$ & 1. 07108400 & 5. 80349400 & -1.29790900 \\
\hline $\mathrm{C}$ & 0.46549700 & 5. 33325400 & -3.70487100 \\
\hline $\mathrm{H}$ & -0.28602000 & -0.83190600 & 1. 88101500 \\
\hline $\mathrm{H}$ & 2. 73728000 & 0.04514400 & -1.21005400 \\
\hline $\mathrm{H}$ & 2. 19418900 & -1.95365600 & -2.48449800 \\
\hline $\mathrm{H}$ & 3. 77494000 & -2.54194800 & -1.84919900 \\
\hline $\mathrm{H}$ & 1. 60686000 & -2.95597800 & 2. 76026800 \\
\hline $\mathrm{H}$ & -0.05915500 & -4.10032800 & 4. 20535500 \\
\hline $\mathrm{H}$ & -2.23449900 & -4.86499800 & 3. 24704800 \\
\hline $\mathrm{H}$ & -2.98437800 & -5.65819700 & 0.31338900 \\
\hline $\mathrm{H}$ & -3.92986800 & -4.32012400 & 0.97562500 \\
\hline $\mathrm{H}$ & -3.34809700 & -2.84360700 & -0.80562400 \\
\hline $\mathrm{H}$ & -3.23056900 & -4.34561900 & -1.73046600 \\
\hline $\mathrm{H}$ & 0.43953300 & -4.82116000 & -1.46231500 \\
\hline $\mathrm{H}$ & -1.08949400 & -5.72039300 & -1.41776000 \\
\hline $\mathrm{H}$ & 0.03037300 & -4.95180700 & -3.83543500 \\
\hline $\mathrm{H}$ & -1.72255800 & -4.84985000 & -3.63124300 \\
\hline $\mathrm{H}$ & -0.28863000 & -2.72234200 & -5.38050100 \\
\hline $\mathrm{H}$ & -0.33261900 & -0.21500600 & -5.21782400 \\
\hline $\mathrm{H}$ & -0.78472200 & 0.85605100 & -3.07708300 \\
\hline $\mathrm{H}$ & -3.78778300 & -0.40545600 & -1.73231100 \\
\hline $\mathrm{H}$ & -6.50377600 & -1.10585700 & 1. 52094600 \\
\hline $\mathrm{H}$ & -2.34625400 & -0.40657700 & 2. 32058600 \\
\hline $\mathrm{H}$ & -3.07792800 & 0.27727100 & 4. 36333200 \\
\hline $\mathrm{H}$ & -2.74408000 & -1.47364200 & 4. 25660200 \\
\hline $\mathrm{H}$ & -3.78738200 & -0.83786300 & 5. 54331000 \\
\hline $\mathrm{H}$ & -6.28276100 & -2.49973600 & 3. 32383300 \\
\hline $\mathrm{H}$ & -5.57419600 & -2.44131600 & 4. 95057400 \\
\hline $\mathrm{H}$ & -4.62938700 & -3.10163800 & 3. 59213900 \\
\hline $\mathrm{H}$ & -5.42856900 & 1. 17664900 & 3. 84564600 \\
\hline $\mathrm{H}$ & -6.04707400 & 0.06840100 & 5. 09565400 \\
\hline $\mathrm{H}$ & -6.75811500 & 0.05561000 & 3. 46913500 \\
\hline $\mathrm{H}$ & -5.67002300 & 0.21809500 & -2.86469300 \\
\hline $\mathrm{H}$ & -6.93670400 & -0.93667700 & -3.31805900 \\
\hline $\mathrm{H}$ & -5.30013500 & -1.52432800 & -2.97155600 \\
\hline $\mathrm{H}$ & -6.28937600 & -3.16057400 & -1.25136500 \\
\hline $\mathrm{H}$ & -7.90025800 & -2.55283600 & -1.70644900 \\
\hline $\mathrm{H}$ & -7.41304800 & -2.57855600 & 0.00073600 \\
\hline $\mathrm{H}$ & -7.20546300 & 1. 09520800 & -0.98882300 \\
\hline
\end{tabular}




\begin{tabular}{|c|c|c|c|}
\hline H & -7.96012700 & -0.03763600 & 0.15680100 \\
\hline $\mathrm{H}$ & -8.43568900 & -0.06192000 & -1.55298100 \\
\hline H & 0.60441000 & 2. 05746000 & -1.45381200 \\
\hline $\mathrm{H}$ & -1.79669200 & 5.55161900 & -2.07911600 \\
\hline $\mathrm{H}$ & -3.61633700 & 1.96208700 & -0.58984900 \\
\hline H & -4.84621700 & 3. 46791300 & 0.59710600 \\
\hline $\mathrm{H}$ & -5.72847600 & 4. 98150400 & 0.30305300 \\
\hline H & -4.04761000 & 5. 03179000 & 0.88966900 \\
\hline $\mathrm{H}$ & -5.19526500 & 6.45314100 & -1.61724100 \\
\hline H & -3.87289700 & 6.10267900 & -2.74528500 \\
\hline H & -3.51511500 & 6.60777400 & -1.07138800 \\
\hline $\mathrm{H}$ & -5.36797400 & 2. 73346000 & -1.84771200 \\
\hline H & -4.92134300 & 3.80052400 & -3.20011300 \\
\hline $\mathrm{H}$ & -6.24084600 & 4. 26077100 & -2.09552800 \\
\hline H & 1. 87056200 & 3. 00478800 & -3.21309900 \\
\hline $\mathrm{H}$ & 2. 31847100 & 3. 35613700 & -1.51907900 \\
\hline H & 2. 86519900 & 4. 42311600 & -2.83272200 \\
\hline H & 0.21976800 & 6.49029500 & -1.17316000 \\
\hline $\mathrm{H}$ & 1. 93808700 & 6.40267600 & -1.62010500 \\
\hline H & 1. 30341400 & 5. 36951000 & -0.31264600 \\
\hline $\mathrm{H}$ & -0.37931300 & 6.03705100 & -3.66244400 \\
\hline H & 0.22752500 & 4. 56603800 & -4.45902700 \\
\hline $\mathrm{H}$ & 1. 34206800 & 5. 89703500 & -4.06172100 \\
\hline $\mathrm{C}$ & 4. 15995100 & -0.84554700 & 0.18326300 \\
\hline $\mathrm{H}$ & 4. 26041900 & -1.79731800 & 0.72364800 \\
\hline $\mathrm{H}$ & 4. 03990100 & -0.08051500 & 0.95870900 \\
\hline $\mathrm{C}$ & 5. 39097700 & -0.53925400 & -0.64976100 \\
\hline $\mathrm{C}$ & 6.34786400 & -1.54862200 & -1.01469100 \\
\hline $\mathrm{C}$ & 5.58396900 & 0.76440300 & -1.08234000 \\
\hline $\mathrm{C}$ & 6.25118800 & -2.91512600 & -0.61314200 \\
\hline $\mathrm{C}$ & 7. 47731200 & -1.16943300 & -1.82505800 \\
\hline $\mathrm{C}$ & 6. 69302500 & 1. 13137900 & -1.87894800 \\
\hline $\mathrm{H}$ & 4. 86103600 & 1.53347000 & -0.79480100 \\
\hline $\mathrm{C}$ & 7. 19911800 & -3.84552600 & -0.98768700 \\
\hline $\mathrm{H}$ & 5. 41796800 & -3.24317700 & 0.01018600 \\
\hline $\mathrm{C}$ & 8. 43631400 & -2.15562700 & -2.19220700 \\
\hline $\mathrm{C}$ & 7. 62075700 & 0.18155500 & -2.24373500 \\
\hline $\mathrm{H}$ & 6.81059600 & 2. 17039900 & -2.19516800 \\
\hline $\mathrm{C}$ & 8.30474600 & -3.46552000 & -1.78651500 \\
\hline $\mathrm{H}$ & 7. 09930800 & -4.88329900 & -0.66007100 \\
\hline $\mathrm{H}$ & 9. 28803300 & -1.85031600 & -2.80574900 \\
\hline $\mathrm{H}$ & 8. 48391200 & 0.45376600 & -2.85659100 \\
\hline $\mathrm{H}$ & 9. 05026500 & -4.21031200 & -2.07454000 \\
\hline $\mathrm{C}$ & 5. 25924800 & 3. 71555500 & 5. 61925000 \\
\hline H & 4. 44714600 & 4. 36915600 & 5. 97472500 \\
\hline
\end{tabular}




$\begin{array}{lrrr}\mathrm{H} & 6.22035300 & 4.18806500 & 5.87075500 \\ \mathrm{H} & 5.18716300 & 2.76344500 & 6.17447800 \\ \mathrm{C} & 5.16614300 & 3.47313100 & 4.11549700 \\ \mathrm{O} & 6.28184000 & 2.73389200 & 3.63551800 \\ \mathrm{C} & 3.84652800 & 2.79918000 & 3.70902600 \\ \mathrm{H} & 3.76330300 & 1.82431500 & 4.21994900 \\ \mathrm{H} & 2.99689000 & 3.40845900 & 4.06189700 \\ \mathrm{C} & 3.75754200 & 2.58600900 & 2.19644100 \\ \mathrm{H} & 3.72832600 & 3.54056800 & 1.64827100 \\ \mathrm{H} & 4.68126900 & 2.07674000 & 1.86656100 \\ \mathrm{C} & 2.59204500 & 1.75569000 & 1.73185700 \\ \mathrm{O} & 2.07527100 & 1.92076200 & 0.58812600 \\ \mathrm{O} & 2.11072000 & 0.83575300 & 2.47846100 \\ \mathrm{H} & 5.23466200 & 4.44504900 & 3.59509700 \\ \mathrm{H} & 6.37906300 & 1.94515300 & 4.18796900 \\ \mathrm{H} & -0.47501700 & 1.09011500 & 2.27720400 \\ \mathrm{H} & -0.36212300 & 1.69247500 & 1.61185700\end{array}$

\section{IntGR2}

$\begin{array}{rrrr}\text { Ir } & 0.38569900 & -0.36783700 & 1.37211800 \\ \text { P } & -1.09737300 & 0.21870400 & -0.33852600 \\ \text { O } & 2.49635300 & -2.91727400 & -1.23048500 \\ \text { N } & 1.73444800 & -1.22119100 & -0.00336500 \\ \text { C } & 2.82499800 & -0.60380500 & -0.82462600 \\ \text { C } & 3.39944200 & -1.83574400 & -1.56525800 \\ \text { C } & 1.64022000 & -2.47122000 & -0.32921100 \\ \text { C } & 0.62226700 & -3.41690900 & 0.17651800 \\ \text { C } & 0.84470400 & -4.10479000 & 1.37990300 \\ \text { C } & -0.14600000 & -4.95047900 & 1.88529400 \\ \text { C } & -1.35733300 & -5.10946900 & 1.20252300 \\ \text { C } & -1.57155300 & -4.42268200 & 0.00451800 \\ \text { C } & -2.75703000 & -4.51158900 & -0.92768800 \\ \text { C } & -2.52242100 & -3.35256900 & -1.91649800 \\ \text { C } & -0.97640300 & -3.10348200 & -1.92162200 \\ \text { C } & -0.58721400 & -3.56855500 & -0.51871100 \\ \text { C } & -0.28057500 & -4.08191800 & -2.92783000 \\ \text { C } & -0.34239300 & -3.38270400 & -4.28335800 \\ \text { C } & -0.33345400 & -1.92342700 & -3.90675300 \\ \text { C } & -0.10525600 & -0.88033600 & -4.80454100 \\ \text { C } & -0.19985500 & 0.43054400 & -4.35305300 \\ \text { C } & -0.50418400 & 0.67292600 & -3.01318800 \\ \text { C } & -0.71643300 & -0.36209000 & -2.07678800 \\ \text { C } & -0.63720100 & -1.71350000 & -2.53869000 \\ \text { C } & -2.85257000 & -0.28009000 & -0.07591400\end{array}$




\begin{tabular}{|c|c|c|c|}
\hline $\mathrm{C}$ & -3.78087100 & 0.00574300 & -1.09310100 \\
\hline $\mathrm{C}$ & -5.13484200 & -0.32615800 & -0.94950400 \\
\hline $\mathrm{C}$ & -5.52533100 & -0.95198000 & 0.24839200 \\
\hline $\mathrm{C}$ & -4.63111600 & -1.25312000 & 1. 28640700 \\
\hline $\mathrm{C}$ & -3.28131500 & -0.90865600 & 1. 09982200 \\
\hline $\mathrm{C}$ & -5.14118800 & -1.93643300 & 2. 57217800 \\
\hline $\mathrm{C}$ & -4.00895600 & -2.19269300 & 3. 58336600 \\
\hline $\mathrm{C}$ & -5.78494800 & -3.29573600 & 2. 21304500 \\
\hline $\mathrm{C}$ & -6.19747300 & -1.02919100 & 3. 24580600 \\
\hline $\mathrm{C}$ & -6.18078900 & -0.04567400 & -2.04805900 \\
\hline $\mathrm{C}$ & -5.57609300 & 0.70649100 & -3.24697300 \\
\hline $\mathrm{C}$ & -6.75276400 & -1.39109800 & -2.55416500 \\
\hline $\mathrm{C}$ & -7.32622100 & 0.81444200 & -1.46593700 \\
\hline $\mathrm{C}$ & -1.16396200 & 2. 07651300 & -0.36295200 \\
\hline $\mathrm{C}$ & 0.00252300 & 2. 81990900 & -0.65686400 \\
\hline $\mathrm{C}$ & -0.00302100 & 4. 21999500 & -0.62048200 \\
\hline $\mathrm{C}$ & -1.19527500 & 4. 86055300 & -0.22037700 \\
\hline $\mathrm{C}$ & -2.35494700 & 4. 16070200 & 0.12121300 \\
\hline $\mathrm{C}$ & -2.32138900 & 2. 75339400 & 0.02920800 \\
\hline $\mathrm{C}$ & -3.65026000 & 4. 86151400 & 0.57783900 \\
\hline $\mathrm{C}$ & -4.00926100 & 4. 38224500 & 2. 00422300 \\
\hline $\mathrm{C}$ & -3.50627900 & 6.39370600 & 0.60451600 \\
\hline $\mathrm{C}$ & -4.79901600 & 4. 49906100 & -0.39194900 \\
\hline $\mathrm{C}$ & 1. 20642200 & 5.07664800 & -1.04985900 \\
\hline $\mathrm{C}$ & 2. 45217100 & 4. 22115600 & -1.33774900 \\
\hline $\mathrm{C}$ & 1.56475500 & 6.09438300 & 0.05697000 \\
\hline $\mathrm{C}$ & 0.83122100 & 5.84073500 & -2.34274500 \\
\hline $\mathrm{H}$ & -0.39689900 & -1.69083300 & 1. 31316000 \\
\hline $\mathrm{H}$ & 2. 33840800 & 0.07806300 & -1.54232400 \\
\hline $\mathrm{H}$ & 3. 41422400 & -1.73066900 & -2.65693100 \\
\hline $\mathrm{H}$ & 4. 39903700 & -2.11991200 & -1.21155700 \\
\hline $\mathrm{H}$ & 1. 77860600 & -3.93977600 & 1. 92152800 \\
\hline $\mathrm{H}$ & 0.02880100 & -5.48474100 & 2. 82187900 \\
\hline $\mathrm{H}$ & -2.12680100 & -5.77737700 & 1. 59921600 \\
\hline $\mathrm{H}$ & -2.76160000 & -5.49112400 & -1.43771500 \\
\hline $\mathrm{H}$ & -3.72193300 & -4.42951100 & -0.40359700 \\
\hline $\mathrm{H}$ & -3.03448600 & -2.45269700 & -1.55524200 \\
\hline $\mathrm{H}$ & -2.90443500 & -3.56073600 & -2.92688500 \\
\hline $\mathrm{H}$ & 0.76921400 & -4.21445200 & -2.62838300 \\
\hline $\mathrm{H}$ & -0.75240500 & -5.07496600 & -2.91613100 \\
\hline $\mathrm{H}$ & 0.49012300 & -3.65370500 & -4.95133500 \\
\hline $\mathrm{H}$ & -1.27256500 & -3.62157900 & -4.82933600 \\
\hline $\mathrm{H}$ & 0.12812700 & -1.09911900 & -5.84980000 \\
\hline $\mathrm{H}$ & -0.04579900 & 1. 27105700 & -5.03372800 \\
\hline $\mathrm{H}$ & -0.59281600 & 1.70959700 & -2.69798600 \\
\hline
\end{tabular}




\begin{tabular}{|c|c|c|c|}
\hline H & -3.43601600 & 0.48721500 & -2.00736900 \\
\hline $\mathrm{H}$ & -6.57592300 & -1.21892700 & 0.37489000 \\
\hline $\mathrm{H}$ & -2.55874300 & -1.15410300 & 1. 87306600 \\
\hline $\mathrm{H}$ & -3.53386500 & -1.25628200 & 3. 91704200 \\
\hline $\mathrm{H}$ & -3.22710300 & -2.85107900 & 3. 17319600 \\
\hline $\mathrm{H}$ & -4.41621800 & -2.68566500 & 4. 47896100 \\
\hline $\mathrm{H}$ & -6.63267200 & -3.18283300 & 1. 52058600 \\
\hline H & -6.16399400 & -3.79121300 & 3. 12099900 \\
\hline $\mathrm{H}$ & -5.05115300 & -3.96958800 & 1. 74265600 \\
\hline H & -5.76656800 & -0.05293600 & 3. 51967300 \\
\hline H & -6.57592500 & -1.50210400 & 4. 16601800 \\
\hline $\mathrm{H}$ & -7.06162700 & -0.84316200 & 2. 59019500 \\
\hline H & -5.15894000 & 1. 68252000 & -2.95264600 \\
\hline $\mathrm{H}$ & -6.35782800 & 0.89667000 & -3.99799700 \\
\hline H & -4.78135000 & 0.12746400 & -3.74287000 \\
\hline $\mathrm{H}$ & -5.95963000 & -2.02182800 & -2.98663500 \\
\hline $\mathrm{H}$ & -7.50952000 & -1.21584300 & -3.33541300 \\
\hline H & -7.23547900 & -1.96351100 & -1.74734300 \\
\hline $\mathrm{H}$ & -6.94891100 & 1. 78015800 & -1.09357300 \\
\hline H & -7.84456900 & 0.31277100 & -0.63490500 \\
\hline $\mathrm{H}$ & -8.07817600 & 1. 02310200 & -2.24340500 \\
\hline $\mathrm{H}$ & 0.90824400 & 2. 29167000 & -0.95493000 \\
\hline $\mathrm{H}$ & -1.20661200 & 5.94834300 & -0.18476000 \\
\hline $\mathrm{H}$ & -3.21846800 & 2. 18533600 & 0.27015600 \\
\hline $\mathrm{H}$ & -4.17573800 & 3. 29475400 & 2. 04575800 \\
\hline $\mathrm{H}$ & -4.93360400 & 4. 87192300 & 2. 34965300 \\
\hline $\mathrm{H}$ & -3.20880200 & 4. 63082200 & 2. 71925000 \\
\hline $\mathrm{H}$ & -4.45198100 & 6.84850600 & 0.93624900 \\
\hline $\mathrm{H}$ & -3.27772100 & 6.80501800 & -0.39104400 \\
\hline $\mathrm{H}$ & -2.72165200 & 6.72399400 & 1. 30310200 \\
\hline $\mathrm{H}$ & -4.99164500 & 3. 41555000 & -0.41794500 \\
\hline $\mathrm{H}$ & -4.57156600 & 4. 82766000 & -1.41843500 \\
\hline $\mathrm{H}$ & -5.73204500 & 4. 99340200 & -0.07803300 \\
\hline $\mathrm{H}$ & 2. 28982100 & 3.52503800 & -2.17557000 \\
\hline $\mathrm{H}$ & 2. 76499100 & 3. 63628200 & -0.45830700 \\
\hline $\mathrm{H}$ & 3. 29657400 & 4. 87029500 & -1.61420300 \\
\hline $\mathrm{H}$ & 0.73694300 & 6.78493000 & 0.27584500 \\
\hline $\mathrm{H}$ & 2. 42544900 & 6.70649200 & -0.25525100 \\
\hline $\mathrm{H}$ & 1. 83683700 & 5.58751100 & 0.99592700 \\
\hline $\mathrm{H}$ & -0.03261900 & 6.50519500 & -2.18985300 \\
\hline $\mathrm{H}$ & 0.57871700 & 5. 14346800 & -3.15738900 \\
\hline $\mathrm{H}$ & 1. 67642800 & 6.46277800 & -2.67813300 \\
\hline $\mathrm{C}$ & 3.88857900 & 0.16651600 & -0.02074500 \\
\hline $\mathrm{H}$ & 4. 11219800 & -0.41922100 & 0.87433100 \\
\hline $\mathrm{H}$ & 3. 44507400 & 1. 10982500 & 0.32145900 \\
\hline
\end{tabular}




$\begin{array}{lrrr}\text { C } & 5.14977500 & 0.44369400 & -0.81993800 \\ \mathrm{C} & 6.37497700 & -0.26739500 & -0.56305900 \\ \mathrm{C} & 5.12124900 & 1.39728300 & -1.82608100 \\ \mathrm{C} & 6.51150800 & -1.26261300 & 0.45350400 \\ \mathrm{C} & 7.53109100 & 0.03475700 & -1.36838100 \\ \mathrm{C} & 6.25800100 & 1.68892200 & -2.61600100 \\ \mathrm{H} & 4.19599800 & 1.94968500 & -2.01168100 \\ \mathrm{C} & 7.71088200 & -1.91600200 & 0.65507500 \\ \mathrm{H} & 5.66578400 & -1.52140400 & 1.09361300 \\ \mathrm{C} & 8.75067500 & -0.65920700 & -1.12820900 \\ \mathrm{C} & 7.43969400 & 1.01913100 & -2.39061100 \\ \mathrm{H} & 6.19347600 & 2.44871800 & -3.39865900 \\ \mathrm{C} & 8.84302500 & -1.61489400 & -0.14027900 \\ \mathrm{H} & 7.78681900 & -2.67177900 & 1.44073400 \\ \mathrm{H} & 9.61886700 & -0.41758200 & -1.74715100 \\ \mathrm{H} & 8.32587600 & 1.23645600 & -2.99260900 \\ \mathrm{H} & 9.78561800 & -2.13964200 & 0.03281700 \\ \mathrm{C} & 2.21487600 & 3.52611400 & 3.31742000 \\ \mathrm{H} & 2.42246800 & 3.82740800 & 4.35500400 \\ \mathrm{H} & 1.25530200 & 3.98771000 & 3.02960600 \\ \mathrm{H} & 3.01191000 & 3.93984300 & 2.67834300 \\ \mathrm{C} & 2.15454000 & 2.00657700 & 3.21009700 \\ \mathrm{O} & 1.72529300 & 1.65440000 & 1.85014800 \\ \mathrm{C} & 3.51192800 & 1.35953800 & 3.53608200 \\ \mathrm{H} & 4.15794800 & 1.45424200 & 2.64821800 \\ \mathrm{H} & 3.98480500 & 1.97955400 & 4.31501000 \\ \mathrm{C} & 3.51581900 & -0.09641600 & 4.05208100 \\ \mathrm{H} & 4.55323000 & -0.42612400 & 4.20491500 \\ \mathrm{H} & 2.99724100 & -0.13518400 & 5.02414700 \\ \mathrm{C} & 2.80917300 & -1.05887300 & 3.10340800 \\ \mathrm{O} & 3.40029200 & -1.90639200 & 2.44717900 \\ \mathrm{O} & 1.52414100 & -0.81844200 & 3.06116700 \\ \mathrm{H} & 1.39042900 & 1.61762200 & 3.90228600 \\ \mathrm{H} & & \\ \mathrm{H} & & 2.42404500 & 1.45381600 \\ \mathrm{H} & & -0.23380300 & 2.64770700 \\ & 0.59508900 & 2.35039500\end{array}$

\section{TSHR1}

$\begin{array}{cccc}\text { Ir } & 0.46875500 & 0.08429400 & 0.82897500 \\ \mathrm{P} & -1.44444800 & 0.27720500 & -0.44076700 \\ 0 & 2.27574300 & -2.30781600 & -2.17760000 \\ \mathrm{~N} & 1.58544500 & -0.73843300 & -0.76098000 \\ \mathrm{C} & 2.69031900 & -0.08185400 & -1.50884300 \\ \mathrm{C} & 2.94094700 & -1.10078400 & -2.64141500\end{array}$




\begin{tabular}{|c|c|c|c|}
\hline $\mathrm{C}$ & 1. 48719600 & -1.96302400 & -1.16565400 \\
\hline $\mathrm{C}$ & 0.59750400 & -2.99012200 & -0.57443300 \\
\hline $\mathrm{C}$ & 1. 02054600 & -3.65999900 & 0.58848600 \\
\hline $\mathrm{C}$ & 0.19725100 & -4.62138800 & 1. 17989800 \\
\hline $\mathrm{C}$ & -1.05406000 & -4.91723000 & 0.62877400 \\
\hline $\mathrm{C}$ & -1.47855200 & -4.23872600 & -0.51484600 \\
\hline $\mathrm{C}$ & -2.75157400 & -4.44061100 & -1.30169300 \\
\hline $\mathrm{C}$ & -2.77606000 & -3.23374100 & -2.26003800 \\
\hline $\mathrm{C}$ & -1.28332900 & -2.78898300 & -2.42815900 \\
\hline $\mathrm{C}$ & -0.66323900 & -3.26834300 & -1.12172100 \\
\hline $\mathrm{C}$ & -0.62114500 & -3.57210200 & -3.60994600 \\
\hline C & -0.95301400 & -2.75809200 & -4.86222900 \\
\hline $\mathrm{C}$ & -1.05164000 & -1.35073000 & -4.32821500 \\
\hline $\mathrm{C}$ & -1.04757900 & -0.19173300 & -5.10434100 \\
\hline $\mathrm{C}$ & -1.19569300 & 1. 04094600 & -4.47586200 \\
\hline $\mathrm{C}$ & -1.33305200 & 1. 09677400 & -3.08813600 \\
\hline $\mathrm{C}$ & -1.32952100 & -0.05983400 & -2.27675200 \\
\hline $\mathrm{C}$ & -1.18838500 & -1.32401700 & -2.91988100 \\
\hline C & -2.98813400 & -0.49339500 & 0.16636300 \\
\hline C & -4.15441800 & -0.38371000 & -0.61075600 \\
\hline $\mathrm{C}$ & -5.37676800 & -0.88589800 & -0.14224700 \\
\hline $\mathrm{C}$ & -5.38084000 & -1.50272600 & 1. 12173100 \\
\hline $\mathrm{C}$ & -4.23540700 & -1.63296300 & 1. 92303800 \\
\hline $\mathrm{C}$ & -3.03111700 & -1.11394300 & 1. 42075300 \\
\hline $\mathrm{C}$ & -4.33200400 & -2.32133200 & 3. 29966500 \\
\hline $\mathrm{C}$ & -2.97297000 & -2.36754000 & 4. 02132000 \\
\hline $\mathrm{C}$ & -4.83283000 & -3.77253400 & 3. 11347100 \\
\hline $\mathrm{C}$ & -5.32772700 & -1.54274700 & 4. 19102100 \\
\hline $\mathrm{C}$ & -6.68534200 & -0.78155600 & -0.95210300 \\
\hline $\mathrm{C}$ & -6.47493600 & -0.08480600 & -2.30856300 \\
\hline $\mathrm{C}$ & -7.23956400 & -2.20080700 & -1.21739100 \\
\hline $\mathrm{C}$ & -7.72168600 & 0.03336000 & -0.14356100 \\
\hline $\mathrm{C}$ & -1.69603600 & 2. 10210800 & -0.26438600 \\
\hline $\mathrm{C}$ & -0.55361600 & 2. 92376100 & -0.38792300 \\
\hline $\mathrm{C}$ & -0.62087900 & 4. 29797100 & -0.14059900 \\
\hline $\mathrm{C}$ & -1.87236800 & 4. 82961100 & 0.23590000 \\
\hline $\mathrm{C}$ & -3.02401400 & 4. 04845700 & 0.37293800 \\
\hline $\mathrm{C}$ & -2.91208300 & 2. 66526000 & 0.12023000 \\
\hline $\mathrm{C}$ & -4.38636500 & 4. 63537500 & 0.79266300 \\
\hline $\mathrm{C}$ & -4.84398800 & 3. 96732600 & 2. 11067900 \\
\hline $\mathrm{C}$ & -4.32201200 & 6.15636200 & 1. 01901700 \\
\hline $\mathrm{C}$ & -5.42674300 & 4. 35449800 & -0.31676500 \\
\hline $\mathrm{C}$ & 0.59762800 & 5. 23383700 & -0.26289300 \\
\hline $\mathrm{C}$ & 1. 87260700 & 4. 47794400 & -0.67665600 \\
\hline $\mathrm{C}$ & 0.86077900 & 5.91246400 & 1. 10189000 \\
\hline
\end{tabular}




\begin{tabular}{|c|c|c|c|}
\hline $\mathrm{C}$ & 0.30798500 & 6.31478000 & -1.33079400 \\
\hline $\mathrm{H}$ & 0.10756300 & -1.33459800 & 1. 27568300 \\
\hline H & 2. 32852000 & 0.87071100 & -1.92498300 \\
\hline $\mathrm{H}$ & 2. 46782000 & -0.80965000 & -3.59076200 \\
\hline $\mathrm{H}$ & 3. 99767200 & -1.33600100 & -2.81292200 \\
\hline $\mathrm{H}$ & 1. 97684900 & -3.40112500 & 1. 04523700 \\
\hline $\mathrm{H}$ & 0.53801200 & -5.13884700 & 2. 07950600 \\
\hline $\mathrm{H}$ & -1.69170200 & -5.67734100 & 1. 08815700 \\
\hline $\mathrm{H}$ & -2.70559200 & -5.39835600 & -1.84982800 \\
\hline $\mathrm{H}$ & -3.64634400 & -4.48881900 & -0.66163900 \\
\hline $\mathrm{H}$ & -3.35043300 & -2.41815100 & -1.80493100 \\
\hline $\mathrm{H}$ & -3.24342200 & -3.45951000 & -3.22980100 \\
\hline $\mathrm{H}$ & 0.46848700 & -3.59519100 & -3.46093100 \\
\hline $\mathrm{H}$ & -0.96941700 & -4.61448600 & -3.65028700 \\
\hline $\mathrm{H}$ & -0.20010800 & -2.85923500 & -5.65941700 \\
\hline $\mathrm{H}$ & -1.91941300 & -3.06113900 & -5.30327100 \\
\hline $\mathrm{H}$ & -0.93896200 & -0.25759700 & -6.19012200 \\
\hline $\mathrm{H}$ & -1.20834400 & 1. 96614100 & -5.05675100 \\
\hline $\mathrm{H}$ & -1.45336100 & 2. 07616600 & -2.62910700 \\
\hline $\mathrm{H}$ & -4.10033100 & 0.09646500 & -1.58758900 \\
\hline $\mathrm{H}$ & -6.32459800 & -1.90083100 & 1. 49849300 \\
\hline $\mathrm{H}$ & -2.11588500 & -1.19209000 & 2. 00325400 \\
\hline $\mathrm{H}$ & -2.57643200 & -1.35872900 & 4. 21694900 \\
\hline $\mathrm{H}$ & -2.21899600 & -2.92988600 & 3. 44844700 \\
\hline $\mathrm{H}$ & -3.08692500 & -2.86813900 & 4. 99492400 \\
\hline $\mathrm{H}$ & -5.82647500 & -3.81003400 & 2. 64175300 \\
\hline $\mathrm{H}$ & -4.91107500 & -4.27816700 & 4. 08910500 \\
\hline $\mathrm{H}$ & -4.13867600 & -4.35348900 & 2. 48538900 \\
\hline $\mathrm{H}$ & -4.99498400 & -0.50380700 & 4. 34405100 \\
\hline $\mathrm{H}$ & -5.40945100 & -2.02173200 & 5. 17976500 \\
\hline $\mathrm{H}$ & -6.33763400 & -1.51185000 & 3. 75480000 \\
\hline $\mathrm{H}$ & -6.10995700 & 0.94790700 & -2.19161700 \\
\hline $\mathrm{H}$ & -7.43080900 & -0.03298000 & -2.85156500 \\
\hline $\mathrm{H}$ & -5.76376300 & -0.63044300 & -2.94861300 \\
\hline $\mathrm{H}$ & -6.52421100 & -2.80436500 & -1.79851800 \\
\hline $\mathrm{H}$ & -8.17787600 & -2.14323600 & -1.79169800 \\
\hline $\mathrm{H}$ & -7.45737000 & -2.74225600 & -0.28424900 \\
\hline $\mathrm{H}$ & -7.35562100 & 1. 05270700 & 0.05824500 \\
\hline $\mathrm{H}$ & -7.95533000 & -0.43720800 & 0.82342400 \\
\hline $\mathrm{H}$ & -8.66481400 & 0.11775300 & -0.70668500 \\
\hline $\mathrm{H}$ & 0.39329700 & 2. 48097400 & -0.70931800 \\
\hline $\mathrm{H}$ & -1.93681900 & 5. 89868000 & 0.43140500 \\
\hline $\mathrm{H}$ & -3.78589900 & 2. 02575700 & 0.23945300 \\
\hline $\mathrm{H}$ & -4.95717200 & 2. 87747800 & 2. 00557600 \\
\hline $\mathrm{H}$ & -5.81884900 & 4. 37321000 & 2. 42494900 \\
\hline
\end{tabular}




\begin{tabular}{|c|c|c|c|}
\hline H & -4.12245700 & 4. 15464500 & 2. 92170100 \\
\hline $\mathrm{H}$ & -5.31513700 & 6.52959600 & 1. 31185900 \\
\hline $\mathrm{H}$ & -4.02474900 & 6.69766700 & 0.10731000 \\
\hline $\mathrm{H}$ & -3.62111000 & 6.42492200 & 1. 82466400 \\
\hline $\mathrm{H}$ & -5.56004300 & 3. 27621500 & -0.49497400 \\
\hline $\mathrm{H}$ & -5.12795500 & 4. 81974300 & -1.26951100 \\
\hline H & -6.40798800 & 4. 76674700 & -0.03219700 \\
\hline $\mathrm{H}$ & 1. 76345100 & 3. 99072200 & -1.65900400 \\
\hline $\mathrm{H}$ & 2. 15649500 & 3.71406700 & 0.06496300 \\
\hline H & 2. 71366200 & 5. 18358200 & -0.75437700 \\
\hline $\mathrm{H}$ & 0.00236900 & 6.51347800 & 1. 43727400 \\
\hline H & 1. 72863600 & 6.58741800 & 1. 03090700 \\
\hline $\mathrm{H}$ & 1. 07452400 & 5. 16527100 & 1. 88258200 \\
\hline $\mathrm{H}$ & -0.56672600 & 6.92930100 & -1.07028300 \\
\hline $\mathrm{H}$ & 0.11816700 & 5. 86037400 & -2.31627800 \\
\hline $\mathrm{H}$ & 1. 17028000 & 6.99319800 & -1.43009500 \\
\hline $\mathrm{C}$ & 3. 89676600 & 0.19609500 & -0.58256400 \\
\hline $\mathrm{H}$ & 4. 15373000 & -0.73124400 & -0.05290700 \\
\hline $\mathrm{H}$ & 3.56225700 & 0.90081300 & 0.19458200 \\
\hline $\mathrm{C}$ & 5. 08804700 & 0.78329300 & -1.31565200 \\
\hline $\mathrm{C}$ & 6.22169100 & -0.01446100 & -1.69993500 \\
\hline $\mathrm{C}$ & 5. 07103700 & 2. 13106200 & -1.64084000 \\
\hline $\mathrm{C}$ & 6.35050900 & -1.40318500 & -1.39617000 \\
\hline $\mathrm{C}$ & 7. 29688500 & 0.61396500 & -2.42420400 \\
\hline $\mathrm{C}$ & 6.12817900 & 2. 74442900 & -2.35219300 \\
\hline $\mathrm{H}$ & 4. 22047200 & 2. 74435900 & -1.32958700 \\
\hline $\mathrm{C}$ & 7. 45942400 & -2.12590100 & -1.78691300 \\
\hline $\mathrm{H}$ & 5.56664100 & -1.91342000 & -0.83459000 \\
\hline $\mathrm{C}$ & 8.42575100 & -0.16236800 & -2.81139000 \\
\hline $\mathrm{C}$ & 7. 21891800 & 1. 99834100 & -2.73862000 \\
\hline $\mathrm{H}$ & 6. 07593100 & 3. 81037200 & -2.58597100 \\
\hline $\mathrm{C}$ & 8. 50918900 & -1.50270400 & -2.50417200 \\
\hline $\mathrm{H}$ & 7. 53124200 & -3.18675300 & -1.53477400 \\
\hline $\mathrm{H}$ & 9. 23320600 & 0.33001300 & -3.35972800 \\
\hline $\mathrm{H}$ & 8. 04343900 & 2. 46090100 & -3.28719900 \\
\hline $\mathrm{H}$ & 9.38271600 & -2.08491700 & -2.80668100 \\
\hline $\mathrm{C}$ & 6. 39295900 & -1.50106100 & 6. 31215200 \\
\hline $\mathrm{H}$ & 5. 76676000 & -2.20013400 & 6. 88917700 \\
\hline $\mathrm{H}$ & 7. 12362800 & -1.05837400 & 7. 00958900 \\
\hline $\mathrm{H}$ & 6. 94943200 & -2.07298700 & 5. 55260800 \\
\hline $\mathrm{C}$ & 5.54882100 & -0.41561300 & 5. 64808700 \\
\hline 0 & 6.35707100 & 0.48739600 & 4. 90398000 \\
\hline $\mathrm{C}$ & 4. 52719200 & -0.99413700 & 4. 66907400 \\
\hline $\mathrm{H}$ & 5.06500000 & -1.56794100 & 3. 89686400 \\
\hline $\mathrm{H}$ & 3. 87943700 & -1.71085200 & 5. 19934300 \\
\hline
\end{tabular}




$\begin{array}{lrrr}\mathrm{C} & 3.68291800 & 0.08539200 & 3.99688700 \\ \mathrm{H} & 3.05978400 & 0.63446000 & 4.72431600 \\ \mathrm{H} & 4.34174100 & 0.84913200 & 3.55048200 \\ \mathrm{C} & 2.77414300 & -0.42817200 & 2.90249800 \\ \mathrm{O} & 2.76337300 & -1.56468800 & 2.48727700 \\ \mathrm{O} & 1.98773300 & 0.54350700 & 2.38486200 \\ \mathrm{H} & 4.99767400 & 0.13735300 & 6.43974700 \\ \mathrm{H} & -0.34603200 & 0.69823000 & 2.17595500 \\ \mathrm{H} & 0.67643200 & 0.60843700 & 2.50766800 \\ \mathrm{H} & 7.00802700 & 0.88083700 & 5.49920500\end{array}$

\section{TSHR2}

\begin{tabular}{|c|c|c|c|}
\hline Ir & -0.34537400 & -0.76213400 & -1.15162400 \\
\hline $\mathrm{P}$ & 1. 06206100 & 0.22744200 & 0.36037900 \\
\hline 0 & -1.72794600 & -3.53529400 & 1. 73924700 \\
\hline $\mathrm{N}$ & -1.37789300 & -1.80578100 & 0.37736700 \\
\hline C & -2.55441300 & -1.38072900 & 1. 18582600 \\
\hline C & -2.70390500 & -2.55720000 & 2. 18443900 \\
\hline C & -1.03087300 & -2.99521600 & 0.75071100 \\
\hline C & 0.08822700 & -3.76371500 & 0.16322300 \\
\hline C & -0.14196700 & -4.55739200 & -0.97100900 \\
\hline C & 0.92637600 & -5.19849800 & -1.60293000 \\
\hline C & 2. 22845200 & -5.04467600 & -1.11592100 \\
\hline C & 2. 45365100 & -4.25556700 & 0.01487300 \\
\hline $\mathrm{C}$ & 3. 75402600 & -4.02223900 & 0.74723500 \\
\hline C & 3. 40948100 & -2.89035200 & 1. 73485600 \\
\hline C & 1. 86653200 & -2.99359600 & 1. 97770400 \\
\hline C & 1. 38959600 & -3.60701800 & 0.66281600 \\
\hline $\mathrm{C}$ & 1. 55867400 & -4.05540000 & 3. 08739100 \\
\hline C & 1. 66342700 & -3.30461700 & 4. 41094500 \\
\hline C & 1. 28096800 & -1.89890600 & 4. 02615600 \\
\hline C & 0.97549400 & -0.89168700 & 4. 94127000 \\
\hline C & 0.72806400 & 0.39136900 & 4. 46979800 \\
\hline C & 0.77217800 & 0.63679600 & 3. 09709600 \\
\hline C & 1. 04786700 & -0.36787100 & 2. 14314100 \\
\hline C & 1. 32393600 & -1.68579600 & 2. 62511900 \\
\hline C & 2. 84642500 & 0.20831000 & -0.09250700 \\
\hline C & 3. 76961200 & 0.73698800 & 0.82724500 \\
\hline C & 5. 13982600 & 0.76414100 & 0.53541900 \\
\hline C & 5. 54830500 & 0.24209900 & -0.70539200 \\
\hline $\mathrm{C}$ & 4. 65793800 & -0.29535600 & -1.64692500 \\
\hline $\mathrm{C}$ & 3. 29201000 & -0.30468500 & -1.31678900 \\
\hline $\mathrm{C}$ & 5. 19010800 & -0.84653700 & -2.98556800 \\
\hline $\mathrm{C}$ & 4. 06372900 & -1.41483700 & -3.86825200 \\
\hline
\end{tabular}




\begin{tabular}{|c|c|c|c|}
\hline $\mathrm{C}$ & 6. 20688000 & -1.97854800 & -2.70952600 \\
\hline $\mathrm{C}$ & 5. 88899200 & 0.29311300 & -3.76328300 \\
\hline $\mathrm{C}$ & 6. 19002700 & 1. 32415800 & 1. 51647900 \\
\hline $\mathrm{C}$ & 5. 54886000 & 1. 87717100 & 2. 80215500 \\
\hline $\mathrm{C}$ & 7. 16991600 & 0.19431900 & 1. 91107400 \\
\hline $\mathrm{C}$ & 6.97283800 & 2. 46983100 & 0.83377200 \\
\hline $\mathrm{C}$ & 0.60305800 & 2. 02664300 & 0.40756700 \\
\hline $\mathrm{C}$ & -0.65875100 & 2. 41719100 & 0.91350900 \\
\hline $\mathrm{C}$ & -1.04107000 & 3. 76463700 & 0.94850000 \\
\hline $\mathrm{C}$ & -0.15120100 & 4. 70942900 & 0.39390500 \\
\hline $\mathrm{C}$ & 1. 08254900 & 4. 35673300 & -0.16195900 \\
\hline $\mathrm{C}$ & 1. 45114600 & 2. 99471800 & -0.13245100 \\
\hline $\mathrm{C}$ & 2. 05004400 & 5. 39077200 & -0.77160100 \\
\hline $\mathrm{C}$ & 2. 35368200 & 5. 00598700 & -2.23858700 \\
\hline $\mathrm{C}$ & 1. 46724600 & 6.81522100 & -0.75746800 \\
\hline $\mathrm{C}$ & 3. 36518700 & 5. 39513900 & 0.04301200 \\
\hline $\mathrm{C}$ & -2.33795100 & 4. 24276200 & 1. 63505800 \\
\hline $\mathrm{C}$ & -3.26466600 & 3. 07159800 & 2. 00542400 \\
\hline $\mathrm{C}$ & -3.12349900 & 5. 20799800 & 0.71913200 \\
\hline $\mathrm{C}$ & -1.94423800 & 4. 98806800 & 2.93405600 \\
\hline $\mathrm{H}$ & 0.70087300 & -1.88794900 & -1.15842700 \\
\hline $\mathrm{H}$ & -2.28136800 & -0.45372500 & 1. 71358800 \\
\hline $\mathrm{H}$ & -2.45875900 & -2.29517500 & 3. 22321900 \\
\hline $\mathrm{H}$ & -3.69481800 & -3.02881600 & 2. 15043900 \\
\hline $\mathrm{H}$ & -1.15276500 & -4.64083900 & -1.37566900 \\
\hline $\mathrm{H}$ & 0.73982700 & -5.81182800 & -2.48715700 \\
\hline $\mathrm{H}$ & 3. 06288800 & -5.54745400 & -1.61217900 \\
\hline $\mathrm{H}$ & 4. 06253500 & -4.94401100 & 1. 27141700 \\
\hline $\mathrm{H}$ & 4. 58266100 & -3.75599000 & 0.07244600 \\
\hline $\mathrm{H}$ & 3. 63869000 & -1.91940400 & 1. 27713700 \\
\hline $\mathrm{H}$ & 3.97468600 & -2.94769200 & 2. 67683100 \\
\hline $\mathrm{H}$ & 0.52944000 & -4.42009700 & 2. 95080500 \\
\hline $\mathrm{H}$ & 2. 22625200 & -4.92565800 & 3. 01122400 \\
\hline $\mathrm{H}$ & 1. 01762000 & -3.72030900 & 5. 20015100 \\
\hline $\mathrm{H}$ & 2. 69316000 & -3.31462200 & 4. 81064300 \\
\hline $\mathrm{H}$ & 0.95407300 & -1.11216400 & 6. 01171700 \\
\hline $\mathrm{H}$ & 0.50876600 & 1. 21053900 & 5. 15861500 \\
\hline $\mathrm{H}$ & 0.59742600 & 1. 65779500 & 2. 76928200 \\
\hline $\mathrm{H}$ & 3. 40999100 & 1. 11990500 & 1. 78128000 \\
\hline $\mathrm{H}$ & 6. 61268100 & 0.25337400 & -0.94597100 \\
\hline $\mathrm{H}$ & 2.56903900 & -0.72585600 & -2.01025700 \\
\hline $\mathrm{H}$ & 3.32390800 & -0.64518300 & -4.13944500 \\
\hline $\mathrm{H}$ & 3.53166200 & -2.24624600 & -3.37949900 \\
\hline $\mathrm{H}$ & 4. 48787000 & -1.80297800 & -4.80686700 \\
\hline $\mathrm{H}$ & 7. 07053600 & -1.62725100 & -2.12490600 \\
\hline
\end{tabular}




\begin{tabular}{|c|c|c|c|}
\hline H & 6.59363900 & -2.38374300 & -3.65810300 \\
\hline $\mathrm{H}$ & 5. 73814700 & -2.80690400 & -2.15439100 \\
\hline $\mathrm{H}$ & 5. 18759600 & 1. 11493800 & -3.97830300 \\
\hline $\mathrm{H}$ & 6.27690000 & -0.08162700 & -4.72393200 \\
\hline H & 6.73886100 & 0.71341900 & -3.20443300 \\
\hline $\mathrm{H}$ & 4. 85398300 & 2. 70603700 & 2. 59391700 \\
\hline $\mathrm{H}$ & 6. 33337100 & 2. 26675000 & 3. 46870000 \\
\hline $\mathrm{H}$ & 5. 00226400 & 1. 10036000 & 3. 35945600 \\
\hline $\mathrm{H}$ & 6.64096300 & -0.63201500 & 2. 41230300 \\
\hline H & 7. 93501800 & 0.57720800 & 2. 60527700 \\
\hline $\mathrm{H}$ & 7. 69429700 & -0.22240400 & 1. 03779000 \\
\hline H & 6. 29969100 & 3. 29052800 & 0.53865200 \\
\hline H & 7. 50428500 & 2. 13008600 & -0.06798100 \\
\hline $\mathrm{H}$ & 7. 72568900 & 2. 88184400 & 1. 52445400 \\
\hline H & -1.31918800 & 1. 66107300 & 1. 33872400 \\
\hline $\mathrm{H}$ & -0.43824900 & 5. 75915900 & 0.41267700 \\
\hline $\mathrm{H}$ & 2. 41882900 & 2. 69458300 & -0.53316000 \\
\hline $\mathrm{H}$ & 2. 82575000 & 4. 01488800 & -2.31915300 \\
\hline $\mathrm{H}$ & 3. 04401000 & 5. 73724900 & -2.68814000 \\
\hline $\mathrm{H}$ & 1. 43395900 & 4. 99408200 & -2.84511500 \\
\hline $\mathrm{H}$ & 2. 18563800 & 7.51315800 & -1.21350100 \\
\hline H & 1. 27002000 & 7. 17302400 & 0.26500700 \\
\hline $\mathrm{H}$ & 0.53166500 & 6.88429800 & -1.33441500 \\
\hline $\mathrm{H}$ & 3. 86171600 & 4. 41276700 & 0.03026100 \\
\hline $\mathrm{H}$ & 3. 17911500 & 5. 66718500 & 1. 09409100 \\
\hline $\mathrm{H}$ & 4. 07100700 & 6. 12947300 & -0.37678500 \\
\hline $\mathrm{H}$ & -2.80273700 & 2. 39187900 & 2. 73829400 \\
\hline $\mathrm{H}$ & -3.56002000 & 2. 47850600 & 1. 12543900 \\
\hline $\mathrm{H}$ & -4.18930900 & 3. 45455500 & 2. 46219600 \\
\hline $\mathrm{H}$ & -2.54351200 & 6. 10463900 & 0.45519700 \\
\hline $\mathrm{H}$ & -4.03593300 & 5.55098100 & 1. 23114400 \\
\hline $\mathrm{H}$ & -3.43032400 & 4. 71532000 & -0.21586800 \\
\hline $\mathrm{H}$ & -1.30981100 & 5. 86353400 & 2. 72685600 \\
\hline $\mathrm{H}$ & -1.39166300 & 4. 32752500 & 3. 62109400 \\
\hline $\mathrm{H}$ & -2.84586700 & 5. 34409700 & 3. 45767700 \\
\hline $\mathrm{C}$ & -3.78481200 & -1.15468900 & 0.30723600 \\
\hline $\mathrm{H}$ & -3.99233700 & -2.09348700 & -0.22988700 \\
\hline $\mathrm{H}$ & -3.51053400 & -0.43563500 & -0.47942800 \\
\hline $\mathrm{C}$ & -5.03388400 & -0.66815300 & 1. 01649700 \\
\hline $\mathrm{C}$ & -6.26000400 & -0.57971500 & 0.26456600 \\
\hline $\mathrm{C}$ & -5.03975600 & -0.28757500 & 2. 34839200 \\
\hline $\mathrm{C}$ & -6.34933200 & -0.95675500 & -1.10810200 \\
\hline $\mathrm{C}$ & -7.44668100 & -0.09431000 & 0.91411700 \\
\hline $\mathrm{C}$ & -6.21447900 & 0.18453700 & 2. 98857600 \\
\hline 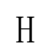 & -4.12578100 & -0.33930500 & 2. 94439100 \\
\hline
\end{tabular}




$\begin{array}{lrrr}\mathrm{C} & -7.54330400 & -0.85369700 & -1.79588200 \\ \mathrm{H} & -5.47375600 & -1.34530700 & -1.63145900 \\ \mathrm{C} & -8.65762700 & -0.00115700 & 0.17309600 \\ \mathrm{C} & -7.39232600 & 0.28121500 & 2.28648500 \\ \mathrm{H} & -6.17399900 & 0.46846700 & 4.04299600 \\ \mathrm{C} & -8.70857600 & -0.37043000 & -1.15421500 \\ \mathrm{H} & -7.59010900 & -1.15645500 & -2.84503500 \\ \mathrm{H} & -9.55346600 & 0.36802600 & 0.67952200 \\ \mathrm{H} & -8.30222700 & 0.64380500 & 2.77170800 \\ \mathrm{H} & -9.64618700 & -0.29680900 & -1.71051500 \\ \mathrm{C} & -2.59793600 & 3.12862600 & -2.68744100 \\ \mathrm{H} & -2.67692200 & 3.64116200 & -3.65775600 \\ \mathrm{H} & -1.82894900 & 3.65246300 & -2.09792900 \\ \mathrm{H} & -3.56664000 & 3.22370000 & -2.17146300 \\ \mathrm{C} & -2.23558800 & 1.66364800 & -2.89483400 \\ \mathrm{O} & -2.01506600 & 1.02823400 & -1.59792100 \\ \mathrm{C} & -3.32816400 & 0.91744800 & -3.66593800 \\ \mathrm{H} & -4.19574400 & 0.78113600 & -2.99878700 \\ \mathrm{H} & -3.66257000 & 1.58237000 & -4.47853800 \\ \mathrm{C} & -2.93544400 & -0.42773500 & -4.30470600 \\ \mathrm{H} & -3.80228200 & -0.81699300 & -4.85814200 \\ \mathrm{H} & -2.10874100 & -0.28127200 & -5.01716800 \\ \mathrm{C} & -2.54399300 & -1.46273200 & -3.26423300 \\ \mathrm{O} & -3.35724300 & -2.14756800 & -2.68280900 \\ \mathrm{O} & -1.23222000 & -1.54554200 & -3.01539600 \\ \mathrm{H} & -1.28448000 & 1.60870600 & -3.45641000 \\ \mathrm{H} & -1.75544700 & 1.72020600 & -0.96606900 \\ \mathrm{H} & -0.24372400 & -0.57635800 & -2.91700400 \\ \mathrm{H} & 0.46983400 & -0.02839200 & -2.45867400\end{array}$

\section{TSHR3}

Ir
P
0
N
C
C
C
C
C
C
C
C
C

$\begin{array}{rrr}-0.62658900 & -0.00511200 & -1.38927900 \\ 0.86358400 & 0.03531600 & 0.45647400 \\ -2.86908800 & -2.87501200 & 0.67526500 \\ -1.99492600 & -1.12715800 & -0.37737700 \\ -3.23140100 & -0.58345300 & 0.26445200 \\ -3.57276800 & -1.72778200 & 1.23568300 \\ -1.93608400 & -2.39953200 & -0.13896300 \\ -0.91695700 & -3.31734100 & -0.69133200 \\ -1.03276700 & -3.77009300 & -2.01718400 \\ -0.04641400 & -4.59045300 & -2.56790500 \\ 1.07118200 & -4.95550400 & -1.80778700 \\ 1.18862600 & -4.50011800 & -0.49350500 \\ 2.25940400 & -4.82759400 & 0.52018100\end{array}$




\begin{tabular}{|c|c|c|c|}
\hline $\mathrm{C}$ & 1. 97779500 & -3.84237000 & 1. 67338100 \\
\hline $\mathrm{C}$ & 0.45974700 & -3.47854600 & 1. 56109800 \\
\hline $\mathrm{C}$ & 0.19937900 & -3.67939700 & 0.07412000 \\
\hline $\mathrm{C}$ & -0.39071200 & -4.54484600 & 2. 33201800 \\
\hline $\mathrm{C}$ & -0.44630700 & -4.04165500 & 3. 77304200 \\
\hline $\mathrm{C}$ & -0.37219500 & -2.54544800 & 3. 60277700 \\
\hline $\mathrm{C}$ & -0.70752900 & -1.61735900 & 4. 58791500 \\
\hline $\mathrm{C}$ & -0.55322200 & -0.26300400 & 4. 31416100 \\
\hline $\mathrm{C}$ & -0.07137500 & 0.13674800 & 3. 06748300 \\
\hline $\mathrm{C}$ & 0.26934700 & -0.77783400 & 2. 04598300 \\
\hline $\mathrm{C}$ & 0.10980000 & -2.17128400 & 2. 32367100 \\
\hline $\mathrm{C}$ & 2. 55519200 & -0.61700000 & 0.16333500 \\
\hline $\mathrm{C}$ & 3. 44284000 & -0.68053200 & 1. 25206000 \\
\hline $\mathrm{C}$ & 4. 76668400 & -1.10748500 & 1. 07873100 \\
\hline $\mathrm{C}$ & 5. 16345400 & -1.46755300 & -0.22124600 \\
\hline $\mathrm{C}$ & 4. 30716500 & -1.41799200 & -1.33211400 \\
\hline $\mathrm{C}$ & 2. 98832300 & -0.98301000 & -1.11699300 \\
\hline $\mathrm{C}$ & 4. 83060800 & -1.83694100 & -2.72161800 \\
\hline $\mathrm{C}$ & 3. 75776000 & -1.69028300 & -3.81541700 \\
\hline $\mathrm{C}$ & 5. 27334600 & -3.31809800 & -2.67744900 \\
\hline $\mathrm{C}$ & 6. 03733600 & -0.94969800 & -3.10627300 \\
\hline $\mathrm{C}$ & 5. 77431200 & -1.19785500 & 2. 24377600 \\
\hline $\mathrm{C}$ & 5. 15270400 & -0.76528100 & 3. 58417600 \\
\hline $\mathrm{C}$ & 6.26311600 & -2.65846400 & 2. 38547900 \\
\hline $\mathrm{C}$ & 6.98196800 & -0.27799100 & 1.94928100 \\
\hline C & 1. 11253700 & 1. 82747200 & 0.84319300 \\
\hline $\mathrm{C}$ & -0.02951500 & 2. 63849300 & 1. 00374100 \\
\hline $\mathrm{C}$ & 0.08587300 & 3. 99926000 & 1. 30291700 \\
\hline $\mathrm{C}$ & 1. 38416900 & 4. 53717700 & 1. 39561900 \\
\hline $\mathrm{C}$ & 2.54217600 & 3. 77745500 & 1. 19608900 \\
\hline $\mathrm{C}$ & 2.38502400 & 2. 40466200 & 0.92472300 \\
\hline $\mathrm{C}$ & 3. 95925700 & 4. 38351400 & 1. 26359200 \\
\hline $\mathrm{C}$ & 4. 67497500 & 4. 15817100 & -0.08882500 \\
\hline $\mathrm{C}$ & 3. 93423500 & 5. 89613400 & 1. 54829800 \\
\hline $\mathrm{C}$ & 4. 75994300 & 3. 69342000 & 2. 39201400 \\
\hline $\mathrm{C}$ & -1.13570800 & 4. 91161700 & 1. 52537700 \\
\hline $\mathrm{C}$ & -2.46243900 & 4. 15531400 & 1. 33174900 \\
\hline $\mathrm{C}$ & -1.09363600 & 6. 08224200 & 0.51463300 \\
\hline $\mathrm{C}$ & -1.09441300 & 5. 46830000 & 2. 96761500 \\
\hline $\mathrm{H}$ & 0.13140600 & -1.29143200 & -1.76179000 \\
\hline $\mathrm{H}$ & -2.97431300 & 0.33230300 & 0.81509800 \\
\hline $\mathrm{H}$ & -3.16821600 & -1.56499900 & 2. 24581100 \\
\hline $\mathrm{H}$ & -4.63883600 & -1.97301400 & 1. 29222000 \\
\hline $\mathrm{H}$ & -1.89739000 & -3.47581100 & -2.61619000 \\
\hline $\mathrm{H}$ & -0.15117700 & -4.94652200 & -3.59521900 \\
\hline
\end{tabular}




\begin{tabular}{|c|c|c|c|}
\hline H & 1. 83943200 & -5.60316700 & -2.23854700 \\
\hline $\mathrm{H}$ & 2. 15894800 & -5.87884100 & 0.84349800 \\
\hline $\mathrm{H}$ & 3. 27749700 & -4.72135300 & 0.11446500 \\
\hline $\mathrm{H}$ & 2.58246300 & -2.93951300 & 1. 53788900 \\
\hline $\mathrm{H}$ & 2. 22318300 & -4.25439700 & 2. 66323800 \\
\hline $\mathrm{H}$ & -1.40630400 & -4.56949100 & 1.91124700 \\
\hline H & 0.03494500 & -5.55326400 & 2. 22627200 \\
\hline $\mathrm{H}$ & -1.35053500 & -4.36453100 & 4. 31188400 \\
\hline $\mathrm{H}$ & 0.41629100 & -4.39578900 & 4. 36547600 \\
\hline H & -1.07773000 & -1.95654800 & 5. 55889500 \\
\hline $\mathrm{H}$ & -0.79841500 & 0.49231900 & 5. 06437100 \\
\hline H & 0.04983800 & 1. 20340900 & 2. 89616300 \\
\hline $\mathrm{H}$ & 3. 08750100 & -0.39790200 & 2. 24234700 \\
\hline $\mathrm{H}$ & 6. 19051700 & -1.80372000 & -0.37297100 \\
\hline H & 2. 28882000 & -0.92771700 & -1.94765400 \\
\hline $\mathrm{H}$ & 3. 42202200 & -0.64637900 & -3.92038500 \\
\hline $\mathrm{H}$ & 2. 87621700 & -2.32006100 & -3.61805400 \\
\hline $\mathrm{H}$ & 4. 17282600 & -1.99939600 & -4.78679200 \\
\hline $\mathrm{H}$ & 6.07315700 & -3.48920700 & -1.94127600 \\
\hline $\mathrm{H}$ & 5. 65701000 & -3.63302900 & -3.66110200 \\
\hline $\mathrm{H}$ & 4. 42802500 & -3.97533900 & -2.41776200 \\
\hline $\mathrm{H}$ & 5. 75181200 & 0.11357500 & -3.14752500 \\
\hline $\mathrm{H}$ & 6.42189700 & -1.23694100 & -4.09789600 \\
\hline $\mathrm{H}$ & 6.86769800 & -1.04592700 & -2.39070000 \\
\hline $\mathrm{H}$ & 4. 80401700 & 0.27904100 & 3. 56100300 \\
\hline $\mathrm{H}$ & 5.90570000 & -0.84012000 & 4. 38340200 \\
\hline $\mathrm{H}$ & 4. 30378700 & -1.40465400 & 3. 87361000 \\
\hline $\mathrm{H}$ & 5. 42342000 & -3.34005300 & 2. 59576600 \\
\hline $\mathrm{H}$ & 6.98152000 & -2.74151000 & 3. 21646600 \\
\hline $\mathrm{H}$ & 6.76875400 & -3.01667400 & 1. 47581800 \\
\hline $\mathrm{H}$ & 6. 66696000 & 0.77271900 & 1. 84945600 \\
\hline $\mathrm{H}$ & 7. 50366000 & -0.56223100 & 1. 02278800 \\
\hline $\mathrm{H}$ & 7. 71339500 & -0.33427500 & 2. 77115000 \\
\hline $\mathrm{H}$ & -1.01949500 & 2. 19733200 & 0.89464100 \\
\hline $\mathrm{H}$ & 1. 48350600 & 5. 59805200 & 1. 61915400 \\
\hline $\mathrm{H}$ & 3. 26787500 & 1. 78597500 & 0.77440000 \\
\hline $\mathrm{H}$ & 4. 77722600 & 3. 08872800 & -0.32992700 \\
\hline $\mathrm{H}$ & 5. 68843500 & 4. 58968300 & -0.06209800 \\
\hline $\mathrm{H}$ & 4. 12382400 & 4. 64025700 & -0.91198600 \\
\hline $\mathrm{H}$ & 4. 96398300 & 6.28346400 & 1.58358900 \\
\hline $\mathrm{H}$ & 3. 46552700 & 6.12654600 & 2. 51761800 \\
\hline $\mathrm{H}$ & 3. 39932600 & 6. 45548900 & 0.76513000 \\
\hline $\mathrm{H}$ & 4. 85235500 & 2. 60886400 & 2. 22718500 \\
\hline $\mathrm{H}$ & 4. 27792900 & 3.84589100 & 3. 37068900 \\
\hline . & 5. 77903900 & 4. 10865100 & 2. 44834900 \\
\hline
\end{tabular}




\begin{tabular}{|c|c|c|c|}
\hline H & -2.58120400 & 3. 33736200 & 2. 06158100 \\
\hline $\mathrm{H}$ & -2.54170900 & 3. 73803700 & 0.31693900 \\
\hline $\mathrm{H}$ & -3.30628900 & 4. 84633000 & 1. 48167700 \\
\hline $\mathrm{H}$ & -0.20171000 & 6.71219900 & 0.65422100 \\
\hline $\mathrm{H}$ & -1.97520100 & 6.72938700 & 0.65039800 \\
\hline $\mathrm{H}$ & -1.08978100 & 5. 70163000 & -0.51664800 \\
\hline H & -0.18315800 & 6. 05579500 & 3. 15787700 \\
\hline $\mathrm{H}$ & -1.13245000 & 4. 65620200 & 3. 71183400 \\
\hline $\mathrm{H}$ & -1.95731400 & 6. 13021800 & 3. 14492600 \\
\hline $\mathrm{C}$ & -4.31942600 & -0.25718300 & -0.78410000 \\
\hline $\mathrm{H}$ & -4.50340000 & -1.15238600 & -1.39613900 \\
\hline $\mathrm{H}$ & -3.91484200 & 0.51547100 & -1.45169400 \\
\hline $\mathrm{C}$ & -5.59794000 & 0.26989800 & -0.15947400 \\
\hline $\mathrm{C}$ & -6.75097200 & -0.56034800 & 0.06211800 \\
\hline $\mathrm{C}$ & -5.64164700 & 1. 60415200 & 0.21664200 \\
\hline $\mathrm{C}$ & -6.81966800 & -1.93629500 & -0.31165100 \\
\hline $\mathrm{C}$ & -7.91275200 & 0.02123600 & 0.68445600 \\
\hline $\mathrm{C}$ & -6.78392600 & 2. 16985500 & 0.82918000 \\
\hline $\mathrm{H}$ & -4.77319200 & 2. 24008100 & 0.02156100 \\
\hline $\mathrm{C}$ & -7.95190700 & -2.69101300 & -0.08052600 \\
\hline $\mathrm{H}$ & -5.96771800 & -2.41108200 & -0.80103200 \\
\hline $\mathrm{C}$ & -9.06343600 & -0.78694800 & 0.90795500 \\
\hline $\mathrm{C}$ & -7.89639900 & 1. 39211400 & 1. 06115600 \\
\hline $\mathrm{H}$ & -6.77926600 & 3. 22611500 & 1. 10820300 \\
\hline $\mathrm{C}$ & -9.08742500 & -2.11374900 & 0.53771100 \\
\hline $\mathrm{H}$ & -7.97538800 & -3.74086900 & -0.38311500 \\
\hline $\mathrm{H}$ & -9.93689200 & -0.32985500 & 1. 38048600 \\
\hline $\mathrm{H}$ & -8.78587800 & 1.81921300 & 1. 53150400 \\
\hline $\mathrm{H}$ & -9.97896500 & -2.72008400 & 0.71376900 \\
\hline $\mathrm{C}$ & 1. 86942800 & 2. 79773200 & -3.96044700 \\
\hline $\mathrm{H}$ & 2. 08697800 & 2. 98936700 & -5.02195900 \\
\hline $\mathrm{H}$ & 2. 81854100 & 2.58430500 & -3.44684900 \\
\hline $\mathrm{H}$ & 1. 43244600 & 3. 71101600 & -3.52728400 \\
\hline $\mathrm{C}$ & 0.91231500 & 1. 62047800 & -3.83059300 \\
\hline 0 & 0.73313900 & 1. 36188500 & -2.38698900 \\
\hline $\mathrm{C}$ & -0.42164500 & 1. 79041000 & -4.57727700 \\
\hline $\mathrm{H}$ & -0.17139200 & 1. 95369200 & -5.63860700 \\
\hline $\mathrm{H}$ & -0.95446300 & 0.82853200 & -4.55417800 \\
\hline $\mathrm{C}$ & -1.35755200 & 2.92452000 & -4.09793700 \\
\hline $\mathrm{H}$ & -0.97439600 & 3. 90678200 & -4.40666200 \\
\hline $\mathrm{H}$ & -2.34710900 & 2. 78108400 & -4.55822900 \\
\hline $\mathrm{C}$ & -1.46885800 & 2.91644400 & -2.57269100 \\
\hline 0 & -0.78657000 & 3. 70751800 & -1.91893700 \\
\hline 0 & -2.17136300 & 1. 95751600 & -2.03597300 \\
\hline $\mathrm{H}$ & 1. 39563500 & 0.70412400 & -4.20053000 \\
\hline
\end{tabular}




$\begin{array}{rrrr}\mathrm{H} & -1.66616200 & -0.22331400 & -2.81965900 \\ \mathrm{H} & -1.93883700 & 0.62114600 & -2.60356600 \\ \mathrm{H} & 0.59474300 & 2.23281000 & -1.94961700\end{array}$

\section{TSHR4}

\begin{tabular}{|c|c|c|c|}
\hline Ir & 0.47580400 & 0.02366900 & 1. 30744200 \\
\hline $\mathrm{P}$ & -1.04303400 & 0.21259900 & -0.41340500 \\
\hline 0 & 2. 70185000 & -2.63343400 & -1.12057300 \\
\hline $\mathrm{N}$ & 1.90350100 & -0.99778900 & 0.15577300 \\
\hline $\mathrm{C}$ & 3. 09550100 & -0.39800100 & -0.52150300 \\
\hline $\mathrm{C}$ & 3. 34085300 & -1.42691600 & -1.63564000 \\
\hline $\mathrm{C}$ & 1.83695700 & -2.24452500 & -0.19344100 \\
\hline $\mathrm{C}$ & 0.87316400 & -3.22504300 & 0.34997000 \\
\hline $\mathrm{C}$ & 1. 08398800 & -3.77574000 & 1. 62552500 \\
\hline $\mathrm{C}$ & 0.14718500 & -4.65305500 & 2. 17479000 \\
\hline $\mathrm{C}$ & -1.01621000 & -4.97556300 & 1. 46601200 \\
\hline $\mathrm{C}$ & -1.22898200 & -4.41991400 & 0.20317500 \\
\hline $\mathrm{C}$ & -2.36706400 & -4.67825400 & -0.75534100 \\
\hline $\mathrm{C}$ & -2.17955600 & -3.59513100 & -1.83744300 \\
\hline $\mathrm{C}$ & -0.66011200 & -3.22082500 & -1.80569600 \\
\hline $\mathrm{C}$ & -0.28946000 & -3.54256700 & -0.36366700 \\
\hline $\mathrm{C}$ & 0.14103700 & -4.20800200 & -2.72132400 \\
\hline $\mathrm{C}$ & 0.08737600 & -3.58876900 & -4.11565700 \\
\hline $\mathrm{C}$ & 0.01679000 & -2.11288400 & -3.81743300 \\
\hline $\mathrm{C}$ & 0.27904700 & -1.10620600 & -4.74632200 \\
\hline $\mathrm{C}$ & 0.13745000 & 0.22027200 & -4.35652800 \\
\hline $\mathrm{C}$ & -0.26090500 & 0.51375800 & -3.05226900 \\
\hline $\mathrm{C}$ & -0.52478500 & -0.48172300 & -2.08526200 \\
\hline $\mathrm{C}$ & -0.37678100 & -1.84880000 & -2.48183100 \\
\hline $\mathrm{C}$ & -2.70340000 & -0.50535600 & -0.08478100 \\
\hline $\mathrm{C}$ & -3.64450400 & -0.47961900 & -1.13003000 \\
\hline $\mathrm{C}$ & -4.95142700 & -0.94739900 & -0.93559300 \\
\hline $\mathrm{C}$ & -5.27957600 & -1.43642800 & 0.34151300 \\
\hline $\mathrm{C}$ & -4.36977000 & -1.47705800 & 1. 40883800 \\
\hline $\mathrm{C}$ & -3.06816500 & -1.00290300 & 1. 17191400 \\
\hline $\mathrm{C}$ & -4.81630300 & -2.02475800 & 2. 77985500 \\
\hline $\mathrm{C}$ & -3.67930400 & -1.98945800 & 3. 81689600 \\
\hline $\mathrm{C}$ & -5.28159400 & -3.49112000 & 2. 62255000 \\
\hline $\mathrm{C}$ & -5.98787800 & -1.16810800 & 3. 31473500 \\
\hline $\mathrm{C}$ & -6.01007800 & -0.95482700 & -2.05801900 \\
\hline $\mathrm{C}$ & -5.47472100 & -0.34430100 & -3.36568500 \\
\hline $\mathrm{C}$ & -6.43679400 & -2.41423900 & -2.34268700 \\
\hline $\mathrm{C}$ & -7.24402500 & -0.13723600 & -1.61002700 \\
\hline $\mathrm{C}$ & -1.36343400 & 2. 02027800 & -0.64800200 \\
\hline
\end{tabular}




\begin{tabular}{|c|c|c|c|}
\hline $\mathrm{C}$ & -0.26627600 & 2. 87988500 & -0.85526400 \\
\hline $\mathrm{C}$ & -0.44232000 & 4. 26007900 & -0.97275600 \\
\hline $\mathrm{C}$ & -1.75209900 & 4. 76611500 & -0.85141300 \\
\hline $\mathrm{C}$ & -2.86385200 & 3. 95031500 & -0.61924600 \\
\hline $\mathrm{C}$ & -2.64766100 & 2. 56151600 & -0.52208300 \\
\hline $\mathrm{C}$ & -4.29403300 & 4. 50922900 & -0.47005500 \\
\hline $\mathrm{C}$ & -4.86431700 & 4. 09975200 & 0.90765500 \\
\hline $\mathrm{C}$ & -4.33401800 & 6.04499300 & -0.56938300 \\
\hline $\mathrm{C}$ & -5.18743800 & 3. 92909900 & -1.59079700 \\
\hline $\mathrm{C}$ & 0.73755100 & 5. 22832000 & -1.18785400 \\
\hline $\mathrm{C}$ & 2. 07372500 & 4. 48379900 & -1.36557200 \\
\hline $\mathrm{C}$ & 0.85565900 & 6. 14946300 & 0.04943200 \\
\hline $\mathrm{C}$ & 0.48634700 & 6. 08447100 & -2.45014600 \\
\hline $\mathrm{H}$ & -0.20460600 & -1.34226500 & 1. 56858600 \\
\hline $\mathrm{H}$ & 2.82555500 & 0.58310000 & -0.93477500 \\
\hline $\mathrm{H}$ & 2. 83100100 & -1.17011500 & -2.57638000 \\
\hline $\mathrm{H}$ & 4. 39529200 & -1.65129200 & -1.82720900 \\
\hline $\mathrm{H}$ & 1. 97952700 & -3.50599800 & 2. 18893900 \\
\hline $\mathrm{H}$ & 0.32501900 & -5.08530500 & 3. 16209900 \\
\hline $\mathrm{H}$ & -1.74703000 & -5.66557800 & 1. 89610300 \\
\hline $\mathrm{H}$ & -2.28034100 & -5.69676900 & -1.17345200 \\
\hline $\mathrm{H}$ & -3.35403600 & -4.62276800 & -0.27053500 \\
\hline $\mathrm{H}$ & -2.77926100 & -2.71539800 & -1.58162500 \\
\hline $\mathrm{H}$ & -2.49484800 & -3.92536600 & -2.83816900 \\
\hline $\mathrm{H}$ & 1. 18464400 & -4.25006900 & -2.37772300 \\
\hline $\mathrm{H}$ & -0.26614700 & -5.22809300 & -2.66880800 \\
\hline $\mathrm{H}$ & 0.95140900 & -3.85473400 & -4.74414900 \\
\hline $\mathrm{H}$ & -0.81448400 & -3.90350000 & -4.67072100 \\
\hline $\mathrm{H}$ & 0.58315400 & -1.36450300 & -5.76402600 \\
\hline $\mathrm{H}$ & 0.32663200 & 1. 03553600 & -5.05859700 \\
\hline $\mathrm{H}$ & -0.38189400 & 1. 56166100 & -2.79103500 \\
\hline $\mathrm{H}$ & -3.34655500 & -0.09232500 & -2.10370500 \\
\hline $\mathrm{H}$ & -6.29344800 & -1.80316300 & 0.51033300 \\
\hline $\mathrm{H}$ & -2.33001700 & -1.02564400 & 1. 96977700 \\
\hline $\mathrm{H}$ & -3.32554300 & -0.96346900 & 4. 00546200 \\
\hline $\mathrm{H}$ & -2.81768200 & -2.60033500 & 3. 50439300 \\
\hline $\mathrm{H}$ & -4.03905900 & -2.39213300 & 4. 77599400 \\
\hline $\mathrm{H}$ & -6.12644000 & -3.58497200 & 1. 92350800 \\
\hline $\mathrm{H}$ & -5.61111800 & -3.89446800 & 3. 59338100 \\
\hline $\mathrm{H}$ & -4.46331000 & -4.12912600 & 2. 25174100 \\
\hline $\mathrm{H}$ & -5.68685700 & -0.11547700 & 3. 43620900 \\
\hline $\mathrm{H}$ & -6.31784200 & -1.54224300 & 4. 29710300 \\
\hline $\mathrm{H}$ & -6.85916400 & -1.19283100 & 2. 64281200 \\
\hline $\mathrm{H}$ & -5.16842100 & 0.70550400 & -3.23655600 \\
\hline $\mathrm{H}$ & -6.26266900 & -0.36386000 & -4.13378200 \\
\hline
\end{tabular}




\begin{tabular}{|c|c|c|c|}
\hline H & -4.61609300 & -0.90711900 & -3.76414900 \\
\hline $\mathrm{H}$ & -5.57980200 & -3.02087400 & -2.67660300 \\
\hline $\mathrm{H}$ & -7.19939100 & -2.44059100 & -3.13733500 \\
\hline $\mathrm{H}$ & -6.86799600 & -2.90007900 & -1.45412800 \\
\hline $\mathrm{H}$ & -6.97296400 & 0.90910800 & -1.39823700 \\
\hline $\mathrm{H}$ & -7.71270500 & -0.55308800 & -0.70532900 \\
\hline H & -8.00732900 & -0.13450700 & -2.40424400 \\
\hline $\mathrm{H}$ & 0.73792800 & 2. 46663300 & -0.89865700 \\
\hline $\mathrm{H}$ & -1.89649200 & 5. 84240900 & -0.93017900 \\
\hline H & -3.49634800 & 1. 90553200 & -0.33741000 \\
\hline $\mathrm{H}$ & -4.91312000 & 3. 00646100 & 1. 02752100 \\
\hline H & -5.88676600 & 4. 49182500 & 1. 03083100 \\
\hline $\mathrm{H}$ & -4.24685200 & 4. 50196900 & 1. 72647900 \\
\hline H & -5.37080300 & 6. 39611600 & -0.45310300 \\
\hline H & -3.97351200 & 6. 40632900 & -1.54498900 \\
\hline $\mathrm{H}$ & -3.73416900 & 6.52544400 & 0.21921400 \\
\hline $\mathrm{H}$ & -5.24180600 & 2. 83048100 & -1.54442800 \\
\hline $\mathrm{H}$ & -4.80528900 & 4. 20604100 & -2.58608900 \\
\hline $\mathrm{H}$ & -6.21512900 & 4. 31677300 & -1.50260900 \\
\hline $\mathrm{H}$ & 2. 05630200 & 3. 81882600 & -2.24466600 \\
\hline $\mathrm{H}$ & 2. 33417000 & 3. 88643400 & -0.47876600 \\
\hline $\mathrm{H}$ & 2. 88379800 & 5. 21200700 & -1.52490800 \\
\hline $\mathrm{H}$ & -0.05214500 & 6. 75389100 & 0.20018200 \\
\hline $\mathrm{H}$ & 1. 70218100 & 6.84495100 & -0.06890400 \\
\hline $\mathrm{H}$ & 1. 02394700 & 5. 55683600 & 0.96192600 \\
\hline $\mathrm{H}$ & -0.43004600 & 6.68862300 & -2.37001600 \\
\hline $\mathrm{H}$ & 0.39488800 & 5. 45234900 & -3.34811400 \\
\hline $\mathrm{H}$ & 1. 32514300 & 6. 78041900 & -2.61112000 \\
\hline $\mathrm{C}$ & 4. 26368300 & -0.22108000 & 0.47862800 \\
\hline $\mathrm{H}$ & 4. 42778900 & -1.17597500 & 1. 00183600 \\
\hline $\mathrm{H}$ & 3.94612500 & 0.53694100 & 1. 21186400 \\
\hline $\mathrm{C}$ & 5.54343000 & 0.28710300 & -0.15903500 \\
\hline $\mathrm{C}$ & 6. 60931600 & -0.59262800 & -0.55678700 \\
\hline $\mathrm{C}$ & 5. 67690300 & 1. 65383800 & -0.35559900 \\
\hline $\mathrm{C}$ & 6.58597000 & -2.00664000 & -0.36198400 \\
\hline $\mathrm{C}$ & 7. 77986700 & -0.02312200 & -1.17323400 \\
\hline $\mathrm{C}$ & 6.82737200 & 2. 20485800 & -0.96677800 \\
\hline $\mathrm{H}$ & 4. 88553400 & 2. 31765400 & 0.00267300 \\
\hline $\mathrm{C}$ & 7. 64089000 & -2.80653800 & -0.75316300 \\
\hline $\mathrm{H}$ & 5. 72322600 & -2.47823100 & 0.11198900 \\
\hline $\mathrm{C}$ & 8. 84798900 & -0.87823500 & -1.56660100 \\
\hline $\mathrm{C}$ & 7.85537400 & 1. 38298800 & -1.37167500 \\
\hline $\mathrm{H}$ & 6.89774500 & 3. 28649500 & -1.10381500 \\
\hline $\mathrm{C}$ & 8. 78525600 & -2.23978900 & -1.36452200 \\
\hline 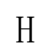 & 7. 59525500 & -3.88548000 & -0.58520200 \\
\hline
\end{tabular}




$\begin{array}{rrrr}\mathrm{H} & 9.72931500 & -0.42953900 & -2.03257500 \\ \mathrm{H} & 8.75003100 & 1.80020200 & -1.84122900 \\ \mathrm{H} & 9.61474400 & -2.88220900 & -1.66905300 \\ \mathrm{C} & 0.50781100 & -0.10581400 & 4.98516500 \\ \mathrm{H} & 0.51277300 & -0.02342200 & 6.08222800 \\ \mathrm{H} & 0.12159300 & -1.10064400 & 4.71877200 \\ \mathrm{H} & -0.18605000 & 0.65331200 & 4.59665000 \\ \mathrm{C} & 1.92219900 & 0.07767600 & 4.46438900 \\ \mathrm{O} & 1.94455900 & -0.28271700 & 3.04109700 \\ \mathrm{C} & 2.53392200 & 1.46595700 & 4.69907100 \\ \mathrm{H} & 2.47827300 & 1.66744400 & 5.78173100 \\ \mathrm{H} & 3.60781500 & 1.43337300 & 4.44871700 \\ \mathrm{C} & 1.90572900 & 2.62777300 & 3.91040300 \\ \mathrm{H} & 0.82671000 & 2.72247700 & 4.10305200 \\ \mathrm{H} & 2.38255800 & 3.56268100 & 4.24311600 \\ \mathrm{C} & 2.18905900 & 2.47289300 & 2.41194500 \\ \mathrm{O} & 3.34946200 & 2.49268800 & 2.01822500 \\ \mathrm{O} & 1.16198500 & 2.27005700 & 1.62770500 \\ \mathrm{H} & 2.56690700 & -0.66808900 & 4.96076400 \\ \mathrm{H} & -0.70603400 & 0.77384400 & 2.28303700 \\ \mathrm{H} & -0.07739000 & 1.46624700 & 2.12182400 \\ \mathrm{H} & 2.84989700 & -0.17913000 & 2.70624800\end{array}$

\section{IntIR}

$\begin{array}{rrrr}\text { Ir } & 0.40479800 & -0.34809100 & 0.80243400 \\ \text { P } & -1.43847700 & 0.40673000 & -0.22955200 \\ \text { O } & 2.40718500 & -1.36745500 & -2.91513600 \\ \mathrm{~N} & 1.70326200 & -0.43889600 & -1.00687100 \\ \mathrm{C} & 2.66402600 & 0.55176200 & -1.54954300 \\ \mathrm{C} & 3.18654000 & -0.14768600 & -2.83077700 \\ \mathrm{C} & 1.63786300 & -1.42781800 & -1.82787500 \\ \mathrm{C} & 0.77705100 & -2.62262200 & -1.65189000 \\ \mathrm{C} & 1.27079300 & -3.70115400 & -0.89863700 \\ \mathrm{C} & 0.47533000 & -4.82746800 & -0.67526700 \\ \mathrm{C} & -0.81708200 & -4.89205800 & -1.20599100 \\ \mathrm{C} & -1.30381900 & -3.82322200 & -1.96122900 \\ \mathrm{C} & -2.61630700 & -3.73474800 & -2.70251200 \\ \mathrm{C} & -2.68489200 & -2.25910600 & -3.14056800 \\ \mathrm{C} & -1.20496800 & -1.74961900 & -3.18479800 \\ \mathrm{C} & -0.52132300 & -2.67627400 & -2.18137900 \\ \mathrm{C} & -0.57767400 & -2.03670000 & -4.59031300 \\ \mathrm{C} & -0.95168200 & -0.83220600 & -5.45375900 \\ \mathrm{C} & -1.04682900 & 0.28382400 & -4.44407000 \\ \mathrm{C} & -1.07684000 & 1.64343400 & -4.75555800\end{array}$




\begin{tabular}{|c|c|c|c|}
\hline $\mathrm{C}$ & -1.22843500 & 2.56776100 & -3.72750300 \\
\hline $\mathrm{C}$ & -1.34456500 & 2. 11979300 & -2.41064700 \\
\hline $\mathrm{C}$ & -1.31064700 & 0.75079600 & -2.06592400 \\
\hline $\mathrm{C}$ & -1.14996200 & -0.19972500 & -3.11790100 \\
\hline $\mathrm{C}$ & -3.01814200 & -0.49602700 & 0.00880300 \\
\hline $\mathrm{C}$ & -4.14452100 & -0.09134600 & -0.72838200 \\
\hline $\mathrm{C}$ & -5.39178600 & -0.69887000 & -0.52681200 \\
\hline $\mathrm{C}$ & -5.46253000 & -1.72679800 & 0.43018900 \\
\hline $\mathrm{C}$ & -4.35805300 & -2.16088300 & 1. 17988900 \\
\hline $\mathrm{C}$ & -3.12775100 & -1.52112100 & 0.95611000 \\
\hline $\mathrm{C}$ & -4.52505400 & -3.30162700 & 2. 20406400 \\
\hline $\mathrm{C}$ & -3.20178000 & -3.64212200 & 2. 91361500 \\
\hline $\mathrm{C}$ & -5.02561100 & -4.57267400 & 1. 47910700 \\
\hline $\mathrm{C}$ & -5.55654000 & -2.88001700 & 3. 27644800 \\
\hline $\mathrm{C}$ & -6.65599400 & -0.28694600 & -1.30890100 \\
\hline $\mathrm{C}$ & -6.37796400 & 0.85423200 & -2.30425700 \\
\hline $\mathrm{C}$ & -7.18629100 & -1.50380900 & -2.10293500 \\
\hline $\mathrm{C}$ & -7.74096600 & 0.19020800 & -0.31539500 \\
\hline C & -1.69986700 & 2. 04583500 & 0.59452100 \\
\hline $\mathrm{C}$ & -0.57229700 & 2. 87545900 & 0.77591600 \\
\hline $\mathrm{C}$ & -0.66214600 & 4. 06871100 & 1. 49946700 \\
\hline $\mathrm{C}$ & -1.91786100 & 4. 40697200 & 2. 04585900 \\
\hline $\mathrm{C}$ & -3.05474700 & 3. 60791700 & 1. 89432700 \\
\hline $\mathrm{C}$ & -2.92202900 & 2.41358300 & 1. 15691400 \\
\hline $\mathrm{C}$ & -4.42134600 & 3. 97409100 & 2.50756400 \\
\hline $\mathrm{C}$ & -4.83951600 & 2. 87071800 & 3. 50801900 \\
\hline $\mathrm{C}$ & -4.38439600 & 5. 31776400 & 3. 25742100 \\
\hline $\mathrm{C}$ & -5.47784500 & 4. 07776900 & 1. 38316900 \\
\hline $\mathrm{C}$ & 0.53800800 & 5. 01016100 & 1. 72399200 \\
\hline $\mathrm{C}$ & 1. 81180900 & 4. 50404800 & 1. 02688600 \\
\hline $\mathrm{C}$ & 0.82454600 & 5. 12022200 & 3. 23980000 \\
\hline $\mathrm{C}$ & 0.20749800 & 6.41152200 & 1. 15862300 \\
\hline $\mathrm{H}$ & -0.08032600 & -1.79361300 & 0.64064800 \\
\hline $\mathrm{H}$ & 2. 09695100 & 1. 45982000 & -1.81611000 \\
\hline $\mathrm{H}$ & 3. 01756400 & 0.42888500 & -3.74928700 \\
\hline $\mathrm{H}$ & 4. 24693500 & -0.42991900 & -2.77287500 \\
\hline $\mathrm{H}$ & 2. 27642300 & -3.64028200 & -0.47810300 \\
\hline $\mathrm{H}$ & 0.86966700 & -5.66124800 & -0.08953700 \\
\hline $\mathrm{H}$ & -1.43446500 & -5.78006700 & -1.04581000 \\
\hline $\mathrm{H}$ & -2.60190800 & -4.42028000 & -3.56842200 \\
\hline $\mathrm{H}$ & -3.47781000 & -4.03134100 & -2.08382100 \\
\hline $\mathrm{H}$ & -3.24456800 & -1.68335600 & -2.39502800 \\
\hline $\mathrm{H}$ & -3.19123300 & -2.11754600 & -4.10699100 \\
\hline $\mathrm{H}$ & 0.51624900 & -2.09161100 & -4.48999500 \\
\hline $\mathrm{H}$ & -0.91931400 & -3.00046000 & -4.99556000 \\
\hline
\end{tabular}




\begin{tabular}{|c|c|c|c|}
\hline H & -0.22072700 & -0.62556100 & -6.25108300 \\
\hline $\mathrm{H}$ & -1.92836700 & -0.97112700 & -5.95121900 \\
\hline $\mathrm{H}$ & -0.99155100 & 1. 97018900 & -5.79532100 \\
\hline $\mathrm{H}$ & -1.26361800 & 3. 63906500 & -3.93919500 \\
\hline $\mathrm{H}$ & -1.47462600 & 2.86620600 & -1.62920700 \\
\hline $\mathrm{H}$ & -4.03788900 & 0.70180700 & -1.46763800 \\
\hline H & -6.42564100 & -2.21224500 & 0.59751400 \\
\hline $\mathrm{H}$ & -2.24348200 & -1.81236700 & 1. 51793300 \\
\hline $\mathrm{H}$ & -2.80735700 & -2.78618400 & 3. 48348600 \\
\hline H & -2.42636200 & -3.96937000 & 2. 20319100 \\
\hline $\mathrm{H}$ & -3.36481200 & -4.46485300 & 3. 62671600 \\
\hline H & -5.99905300 & -4.41494300 & 0.99055300 \\
\hline $\mathrm{H}$ & -5.14693500 & -5.40033200 & 2. 19627100 \\
\hline H & -4.30939500 & -4.89614400 & 0.70672500 \\
\hline H & -5.22432200 & -1.97659900 & 3. 81217400 \\
\hline $\mathrm{H}$ & -5.68996400 & -3.68463200 & 4. 01740300 \\
\hline H & -6.54388300 & -2.66694300 & 2. 83927900 \\
\hline $\mathrm{H}$ & -6.02360800 & 1. 76673900 & -1.79933700 \\
\hline $\mathrm{H}$ & -7.30476000 & 1. 11491700 & -2.83781900 \\
\hline $\mathrm{H}$ & -5.63194200 & 0.56953500 & -3.06265600 \\
\hline $\mathrm{H}$ & -6.43581600 & -1.86588700 & -2.82375400 \\
\hline $\mathrm{H}$ & -8.09123800 & -1.22687300 & -2.66710600 \\
\hline $\mathrm{H}$ & -7.45212900 & -2.34423000 & -1.44384000 \\
\hline $\mathrm{H}$ & -7.39444600 & 1. 06097400 & 0.26373300 \\
\hline $\mathrm{H}$ & -8.02324200 & -0.59765300 & 0.39906800 \\
\hline $\mathrm{H}$ & -8.65264000 & 0.48656400 & -0.85845300 \\
\hline $\mathrm{H}$ & 0.37907900 & 2. 58454300 & 0.32420200 \\
\hline $\mathrm{H}$ & -1.99830400 & 5. 33170800 & 2. 61467700 \\
\hline $\mathrm{H}$ & -3.78554100 & 1. 76110600 & 1. 03516300 \\
\hline $\mathrm{H}$ & -4.92879600 & 1. 88608100 & 3. 02411300 \\
\hline $\mathrm{H}$ & -5.81742600 & 3. 11151900 & 3. 95513600 \\
\hline $\mathrm{H}$ & -4.10671700 & 2. 77839100 & 4. 32531900 \\
\hline $\mathrm{H}$ & -5.37957800 & 5.53704700 & 3. 67344600 \\
\hline $\mathrm{H}$ & -4.11509200 & 6.15400500 & 2. 59336900 \\
\hline $\mathrm{H}$ & -3.67443100 & 5.30246300 & 4. 09895300 \\
\hline $\mathrm{H}$ & -5.59506700 & 3. 12888800 & 0.83756700 \\
\hline $\mathrm{H}$ & -5.20558200 & 4. 85630600 & 0.65298300 \\
\hline $\mathrm{H}$ & -6.46078200 & 4. 33973100 & 1. 80645800 \\
\hline $\mathrm{H}$ & 1. 67447400 & 4. 42279100 & -0.06311500 \\
\hline $\mathrm{H}$ & 2. 13183500 & 3.52209300 & 1. 41063100 \\
\hline $\mathrm{H}$ & 2. 64042100 & 5. 20668300 & 1. 20254000 \\
\hline $\mathrm{H}$ & -0.03559100 & 5.52597500 & 3. 79318000 \\
\hline $\mathrm{H}$ & 1. 68074600 & 5. 78989500 & 3. 42031400 \\
\hline $\mathrm{H}$ & 1. 06561700 & 4. 13525600 & 3. 67063100 \\
\hline . & -0.66938400 & 6.85993100 & 1. 64893400 \\
\hline
\end{tabular}




$\begin{array}{rrrr}\mathrm{H} & 0.00058600 & 6.36486600 & 0.07753000 \\ \mathrm{H} & 1.05715900 & 7.09592200 & 1.31251800 \\ \mathrm{C} & 3.75838800 & 0.92742900 & -0.53111400 \\ \mathrm{H} & 4.15274200 & 0.00067200 & -0.10000700 \\ \mathrm{H} & 3.27660000 & 1.47494900 & 0.29637500 \\ \mathrm{C} & 4.86079400 & 1.78600500 & -1.12484800 \\ \mathrm{C} & 6.16934100 & 1.25822800 & -1.40670600 \\ \mathrm{C} & 4.59090800 & 3.11258800 & -1.42485700 \\ \mathrm{C} & 6.55750700 & -0.08670800 & -1.12449900 \\ \mathrm{C} & 7.15278000 & 2.12695100 & -2.00212300 \\ \mathrm{C} & 5.55883400 & 3.96212800 & -2.00897900 \\ \mathrm{H} & 3.60286400 & 3.52132000 & -1.19624900 \\ \mathrm{C} & 7.82598200 & -0.54501700 & -1.41725300 \\ \mathrm{H} & 5.84942600 & -0.77432500 & -0.65975700 \\ \mathrm{C} & 8.45161700 & 1.61953600 & -2.28934300 \\ \mathrm{C} & 6.81528200 & 3.47683200 & -2.29424700 \\ \mathrm{H} & 5.30437100 & 5.00190700 & -2.22782600 \\ \mathrm{C} & 8.78538500 & 0.31316700 & -2.00721700 \\ \mathrm{H} & 8.09422600 & -1.57937100 & -1.18798200 \\ \mathrm{H} & 9.18527000 & 2.29224300 & -2.74138000 \\ \mathrm{H} & 7.57235200 & 4.12327900 & -2.74564100 \\ \mathrm{H} & & & \\ \mathrm{H} & & & \\ \mathrm{H} & & & \end{array}$




\section{IntJR}

Ir

$\mathrm{P}$

0

N

C

C

C

C

C

C

C

C

C

C

C

C

C

C

C

C

C

C

C

C

C

C

$\mathrm{C}$

C

C

C

C

C

C

C

C

C

C

C

C

C

C

C

C

C
0. 43920700

$-1.23575000$

2. 12002000

1. 77699800

2. 91252000

2. 84993000

1. 46847100

0. 45765300

0. 73956200

$-0.21116100$

$-1.46353200$

$-1.75234000$

$-3.00711000$

$-2.84316000$

$-1.30575700$

$-0.80259500$

$-0.68961100$

$-0.83933400$

$-0.76442300$

$-0.51705400$

$-0.51184100$

$-0.76217300$

$-1.01749300$

$-1.00174700$

$-2.94389600$

$-3.98347500$

$-5.29782400$

$-5.53435800$

$-4.52292400$

$-3.22066000$

$-4.86129200$

$-3.61645100$

$-5.46865800$

$-5.88444400$

$-6.46357400$

$-6.01005800$

$-7.06559900$

$-7.55493200$

$-1.47815900$

$-0.32323700$

$-0.40999300$

$-1.69240600$

$-2.86089800$

$-2.73063900$
0. 20042200

0. 11801200

$-3.26233500$

$-1.31540200$

$-1.06009900$

$-2.28699500$

$-2.55631000$

$-3.24590400$

$-3.48240100$

$-4.08168300$

$-4.43907700$

-4. 18762100

$-4.53572100$

$-3.76432800$

$-3.54362700$

$-3.58438800$

$-4.76699800$

$-4.43508600$

$-2.92826300$

$-2.15455000$

$-0.76951800$

$-0.19130400$

$-0.94289400$

$-2.36707600$

$-0.30338100$

$-0.51506200$

$-0.75019000$

$-0.76328000$

$-0.55640100$

$-0.32394100$

$-0.58790700$

$-0.36675100$

$-1.96271500$

0. 52667500

$-1.00242700$

$-0.95193800$

$-2.40144100$

0.07341300

1. 85573100

2. 61034200

3. 93951300

4. 51451800

3. 80625800

2. 46284600
1. 18760600

$-0.31311300$

$-0.86509800$

0. 18837600

$-0.72569900$

$-1.65479400$

0. 08098100

0. 91343000

2. 26866300

3. 09643500

2. 58522800

1. 24307900

0. 47696800

$-0.84802100$

$-1.03532600$

0. 40231100

$-1.79248300$

$-3.27624500$

$-3.28593600$

$-4.41864000$

$-4.29218300$

$-3.04726700$

$-1.87869800$

$-2.00635800$

0. 27003900

$-0.65137000$

$-0.22207000$

1. 16366500

2. 11421800

1. 64185600

3. 61920100

4. 49760400

3. 98264000

3. 93834100

$-1.20229500$

$-2.67270100$

$-0.93329600$

$-0.99683800$

$-0.97188800$

$-1.25942300$

$-1.68525400$

$-1.79077600$

$-1.49715500$

$-1.09238900$ 


\begin{tabular}{|c|c|c|c|}
\hline $\mathrm{C}$ & -4.26582100 & 4. 43714800 & -1.59457300 \\
\hline $\mathrm{C}$ & -4.96544100 & 4. 35059900 & -0.21824600 \\
\hline $\mathrm{C}$ & -4.21623600 & 5. 91773100 & -2.01327500 \\
\hline $\mathrm{C}$ & -5.10147400 & 3. 66942800 & -2.64477400 \\
\hline $\mathrm{C}$ & 0.83802500 & 4. 79538500 & -1.98689900 \\
\hline $\mathrm{C}$ & 2. 12724200 & 3. 95376000 & -2.00944900 \\
\hline $\mathrm{C}$ & 0.97785900 & 5.87006400 & -0.88275400 \\
\hline $\mathrm{C}$ & 0.69067000 & 5. 48733200 & -3.36115700 \\
\hline $\mathrm{H}$ & -0.37305000 & -0.87612600 & 1.95510100 \\
\hline $\mathrm{H}$ & 2. 73284700 & -0.12395000 & -1.26958700 \\
\hline $\mathrm{H}$ & 2. 26699200 & -2.09688200 & -2.56985400 \\
\hline $\mathrm{H}$ & 3. 82530900 & -2.71404700 & -1.91803400 \\
\hline $\mathrm{H}$ & 1. 70503300 & -3.17450800 & 2. 67475500 \\
\hline $\mathrm{H}$ & 0.02412500 & -4.26020900 & 4. 14836100 \\
\hline $\mathrm{H}$ & -2.20855800 & -4.91143400 & 3. 23175200 \\
\hline $\mathrm{H}$ & -3.05893700 & -5.62759200 & 0.31388700 \\
\hline $\mathrm{H}$ & -3.92629700 & -4.25422100 & 1. 01504400 \\
\hline $\mathrm{H}$ & -3.34083200 & -2.79337400 & -0.76423600 \\
\hline $\mathrm{H}$ & -3.28506300 & -4.28570900 & -1.71090700 \\
\hline $\mathrm{H}$ & 0.37711900 & -4.84180200 & -1.54057400 \\
\hline $\mathrm{H}$ & -1.17253900 & -5.70948500 & -1.49314800 \\
\hline $\mathrm{H}$ & -0.06678500 & -4.90351800 & -3.90680600 \\
\hline $\mathrm{H}$ & -1.81578400 & -4.76694000 & -3.67443400 \\
\hline $\mathrm{H}$ & -0.33359400 & -2.63474200 & -5.38389400 \\
\hline $\mathrm{H}$ & -0.31983000 & -0.12816700 & -5.15617300 \\
\hline $\mathrm{H}$ & -0.76090400 & 0.89488100 & -2.98876700 \\
\hline $\mathrm{H}$ & -3.75280400 & -0.49738500 & -1.71610300 \\
\hline $\mathrm{H}$ & -6.55180700 & -0.94520900 & 1. 51588600 \\
\hline $\mathrm{H}$ & -2.39884600 & -0.15182700 & 2. 33351400 \\
\hline $\mathrm{H}$ & -3.14705300 & 0.61109200 & 4. 31073300 \\
\hline $\mathrm{H}$ & -2.85366800 & -1.14330300 & 4. 33389800 \\
\hline $\mathrm{H}$ & -3.90252900 & -0.39908800 & 5. 56087900 \\
\hline $\mathrm{H}$ & -6.39721500 & -2.16700700 & 3. 42716500 \\
\hline $\mathrm{H}$ & -5.70988900 & -2.00389500 & 5. 05761300 \\
\hline $\mathrm{H}$ & -4.75885900 & -2.77634000 & 3. 76307000 \\
\hline $\mathrm{H}$ & -5.47246900 & 1.51954800 & 3. 69785800 \\
\hline $\mathrm{H}$ & -6.14259200 & 0.51668200 & 5. 01018100 \\
\hline $\mathrm{H}$ & -6.81948500 & 0.40435300 & 3. 36995200 \\
\hline $\mathrm{H}$ & -5.59588800 & 0.03154800 & -2.94381600 \\
\hline $\mathrm{H}$ & -6.87042900 & -1.14031300 & -3.33402000 \\
\hline $\mathrm{H}$ & -5.24838000 & -1.71497700 & -2.89595000 \\
\hline $\mathrm{H}$ & -6.31010700 & -3.19053400 & -1.07618700 \\
\hline $\mathrm{H}$ & -7.90043700 & -2.60117800 & -1.62512800 \\
\hline $\mathrm{H}$ & -7.45473700 & -2.49210700 & 0.09221900 \\
\hline $\mathrm{H}$ & -7.15719200 & 1. 08197400 & -1.19199900 \\
\hline
\end{tabular}




\begin{tabular}{|c|c|c|c|}
\hline H & -7.95527300 & 0.06424700 & 0.02829600 \\
\hline $\mathrm{H}$ & -8.39882100 & -0.09771100 & -1.68543100 \\
\hline H & 0.65288100 & 2. 15154800 & -1.11644600 \\
\hline $\mathrm{H}$ & -1.76818800 & 5.55530000 & -2.10208200 \\
\hline $\mathrm{H}$ & -3.62722300 & 1.89517600 & -0.85039700 \\
\hline H & -5.08189800 & 3.31008100 & 0.12028900 \\
\hline $\mathrm{H}$ & -5.97108300 & 4. 80051600 & -0.26815600 \\
\hline H & -4.38885300 & 4. 88892600 & 0.55068600 \\
\hline $\mathrm{H}$ & -5.23904200 & 6. 32313800 & -2.06739100 \\
\hline H & -3.75622000 & 6.05087700 & -3.00491300 \\
\hline H & -3.65582400 & 6.53103400 & -1.29043200 \\
\hline $\mathrm{H}$ & -5.20178600 & 2. 60425400 & -2.38668800 \\
\hline H & -4.63577700 & 3. 72983000 & -3.64147100 \\
\hline $\mathrm{H}$ & -6.11683900 & 4. 09351200 & -2.71696600 \\
\hline $\mathrm{H}$ & 2. 08576800 & 3. 17475000 & -2.78835200 \\
\hline $\mathrm{H}$ & 2. 31839100 & 3. 46316100 & -1.04403900 \\
\hline H & 2.98725800 & 4. 60366900 & -2.23891000 \\
\hline H & 0.09189600 & 6.52258200 & -0.83455300 \\
\hline $\mathrm{H}$ & 1. 85658900 & 6.50851100 & -1.07419100 \\
\hline H & 1. 10486200 & 5. 39892900 & 0.10427000 \\
\hline $\mathrm{H}$ & -0.17162000 & 6.17032400 & -3.39920900 \\
\hline H & 0.56841800 & 4. 74612000 & -4.16744500 \\
\hline $\mathrm{H}$ & 1.59032700 & 6.08414700 & -3.58404400 \\
\hline $\mathrm{C}$ & 4. 22971500 & -0.93768000 & 0.07401500 \\
\hline $\mathrm{H}$ & 4. 44740300 & -1.90660900 & 0.54579000 \\
\hline $\mathrm{H}$ & 4. 04800600 & -0.23850900 & 0.89959400 \\
\hline $\mathrm{C}$ & 5. 38891300 & -0.43611000 & -0.76466400 \\
\hline $\mathrm{C}$ & 6.42889300 & -1.29648300 & -1.25936400 \\
\hline $\mathrm{C}$ & 5. 42219000 & 0.91358200 & -1.08536800 \\
\hline $\mathrm{C}$ & 6. 49898000 & -2.69315100 & -0.97262700 \\
\hline $\mathrm{C}$ & 7. 46648200 & -0.73164200 & -2.08452900 \\
\hline $\mathrm{C}$ & 6. 44330800 & 1. 46374800 & -1.89398800 \\
\hline $\mathrm{H}$ & 4. 63321900 & 1. 56544700 & -0.69892200 \\
\hline $\mathrm{C}$ & 7. 51428000 & -3.48331600 & -1.47137500 \\
\hline $\mathrm{H}$ & 5. 73995300 & -3.15276100 & -0.33835800 \\
\hline $\mathrm{C}$ & 8. 49893900 & -1.57661000 & -2.58256500 \\
\hline $\mathrm{C}$ & 7. 44490700 & 0.65704500 & -2.38711100 \\
\hline $\mathrm{H}$ & 6. 43138800 & 2. 53257800 & -2.12177900 \\
\hline $\mathrm{C}$ & 8.52625700 & -2.92221600 & -2.28816900 \\
\hline $\mathrm{H}$ & 7. 54040800 & -4.54912700 & -1.23035600 \\
\hline $\mathrm{H}$ & 9. 27830900 & -1.13209200 & -3.20792300 \\
\hline $\mathrm{H}$ & 8. 23888800 & 1. 07204000 & -3.01398100 \\
\hline $\mathrm{H}$ & 9.32638200 & -3.55648400 & -2.67799600 \\
\hline $\mathrm{C}$ & 5. 19507400 & 2. 93635300 & 6. 08352200 \\
\hline H & 4. 32824500 & 3. 42317200 & 6. 55876700 \\
\hline
\end{tabular}




$\begin{array}{lrrr}\mathrm{H} & 6.10395800 & 3.44601900 & 6.44683500 \\ \mathrm{H} & 5.23448100 & 1.88759400 & 6.41874200 \\ \mathrm{C} & 5.10383900 & 3.00107200 & 4.55939100 \\ \mathrm{O} & 6.23276600 & 2.37863000 & 3.95474600 \\ \mathrm{C} & 3.86111000 & 2.29519400 & 4.02143600 \\ \mathrm{H} & 3.89361800 & 1.23658400 & 4.32511900 \\ \mathrm{H} & 2.96702000 & 2.72849000 & 4.49923900 \\ \mathrm{C} & 3.73495700 & 2.37747600 & 2.50087600 \\ \mathrm{H} & 3.63727500 & 3.42033500 & 2.15628300 \\ \mathrm{H} & 4.66298400 & 1.99630900 & 2.04096300 \\ \mathrm{C} & 2.57634600 & 1.59495200 & 1.91521200 \\ \mathrm{O} & 2.03517200 & 0.66812800 & 2.59653100 \\ \mathrm{O} & 2.16519200 & 1.83814400 & 0.74676300 \\ \mathrm{H} & 5.05729800 & 4.06930800 & 4.25403200 \\ \mathrm{H} & -0.46149500 & 1.28212800 & 1.91680900 \\ \mathrm{H} & 7.02688300 & 2.85099200 & 4.23463500\end{array}$


The Cartesian coordinates $(\AA)$ for the optimized structures in supporting information.

\section{IntKR1}

\begin{tabular}{|c|c|c|c|}
\hline Ir & -0.54254100 & -0.26058200 & -1.47168900 \\
\hline $\mathrm{P}$ & 0.85907200 & 0.07623800 & 0.38734500 \\
\hline 0 & -2.96194500 & -2.97001600 & 0.79407000 \\
\hline $\mathrm{N}$ & -2.02289200 & -1.30957900 & -0.36824900 \\
\hline $\mathrm{C}$ & -3.26954300 & -0.71247100 & 0.18835700 \\
\hline $\mathrm{C}$ & -3.66840700 & -1.77208900 & 1. 22987000 \\
\hline $\mathrm{C}$ & -1.99484300 & -2.55139800 & -0.03121300 \\
\hline $\mathrm{C}$ & -0.98156700 & -3.51604500 & -0.51279400 \\
\hline $\mathrm{C}$ & -1.15620900 & -4.12042800 & -1.76711800 \\
\hline $\mathrm{C}$ & -0.16688800 & -4.95073900 & -2.29735000 \\
\hline $\mathrm{C}$ & 1. 01145500 & -5.18254000 & -1.58058800 \\
\hline $\mathrm{C}$ & 1. 18066500 & -4.58965700 & -0.32811300 \\
\hline $\mathrm{C}$ & 2. 31822800 & -4.78459300 & 0.64612100 \\
\hline $\mathrm{C}$ & 2. 05091200 & -3.72047700 & 1. 72872800 \\
\hline $\mathrm{C}$ & 0.51832200 & -3.41127200 & 1. 66338400 \\
\hline $\mathrm{C}$ & 0.19349100 & -3.74838900 & 0.21151700 \\
\hline $\mathrm{C}$ & -0.27846600 & -4.44328300 & 2. 53082100 \\
\hline $\mathrm{C}$ & -0.26763300 & -3.87640700 & 3. 94596000 \\
\hline $\mathrm{C}$ & -0.20376700 & -2.38997900 & 3. 70328000 \\
\hline $\mathrm{C}$ & -0.45399700 & -1.43001500 & 4. 68241600 \\
\hline $\mathrm{C}$ & -0.27917800 & -0.09032900 & 4. 36198500 \\
\hline $\mathrm{C}$ & 0.12351800 & 0.25848700 & 3. 07272000 \\
\hline $\mathrm{C}$ & 0.35562900 & -0.68324600 & 2. 04723500 \\
\hline $\mathrm{C}$ & 0.19231200 & -2.06640800 & 2. 38037100 \\
\hline $\mathrm{C}$ & 2.59847300 & -0.50198600 & 0.17186900 \\
\hline $\mathrm{C}$ & 3. 45723500 & -0.48545200 & 1. 28421000 \\
\hline $\mathrm{C}$ & 4. 79726700 & -0.87667000 & 1. 16453500 \\
\hline $\mathrm{C}$ & 5. 23937500 & -1.28799900 & -0.10564800 \\
\hline $\mathrm{C}$ & 4. 41140200 & -1.30670000 & -1.23826200 \\
\hline $\mathrm{C}$ & 3. 07634100 & -0.89615300 & -1.08219200 \\
\hline $\mathrm{C}$ & 4. 98137000 & -1.75446500 & -2.60013400 \\
\hline $\mathrm{C}$ & 3. 90006100 & -1.78438100 & -3.69546700 \\
\hline $\mathrm{C}$ & 5.57871200 & -3.17493000 & -2.47302000 \\
\hline $\mathrm{C}$ & 6. 09036500 & -0.76865900 & -3.03569800 \\
\hline $\mathrm{C}$ & 5. 77531200 & -0.88313500 & 2. 35853300 \\
\hline $\mathrm{C}$ & 5. 11677600 & -0.36926800 & 3. 65193400 \\
\hline $\mathrm{C}$ & 6. 26992100 & -2.32695800 & 2. 60843500 \\
\hline $\mathrm{C}$ & 6.98559400 & 0.02525300 & 2. 04211400 \\
\hline $\mathrm{C}$ & 1. 05605000 & 1.89926600 & 0.67738900 \\
\hline $\mathrm{C}$ & -0.03752400 & 2. 68603400 & 1. 09273700 \\
\hline $\mathrm{C}$ & 0.09855000 & 4. 06329100 & 1. 29426500 \\
\hline $\mathrm{C}$ & 1. 34554700 & 4. 64925800 & 0.99614800 \\
\hline
\end{tabular}




\begin{tabular}{|c|c|c|c|}
\hline $\mathrm{C}$ & 2. 43766300 & 3.91080700 & 0.53124000 \\
\hline $\mathrm{C}$ & 2. 27480800 & 2.51945800 & 0.38876800 \\
\hline $\mathrm{C}$ & 3. 79786200 & 4. 55478700 & 0.18907400 \\
\hline $\mathrm{C}$ & 4. 14841800 & 4. 26379400 & -1.28809000 \\
\hline $\mathrm{C}$ & 3. 78856100 & 6.08125000 & 0.38715000 \\
\hline $\mathrm{C}$ & 4. 89194500 & 3.95775500 & 1. 10438600 \\
\hline $\mathrm{C}$ & -1.04439800 & 4. 94446100 & 1. 84248300 \\
\hline $\mathrm{C}$ & -2.30244000 & 4. 12126300 & 2. 17457000 \\
\hline $\mathrm{C}$ & -1.43281600 & 6.00916600 & 0.78952200 \\
\hline $\mathrm{C}$ & -0.56365300 & 5. 64236800 & 3. 13661700 \\
\hline $\mathrm{H}$ & 0.34461600 & -1.54407100 & -1.71037200 \\
\hline $\mathrm{H}$ & -3.02851400 & 0.25494300 & 0.64315500 \\
\hline $\mathrm{H}$ & -3.30618700 & -1.53007500 & 2. 24110600 \\
\hline $\mathrm{H}$ & -4.74010100 & -1.99889500 & 1. 26553700 \\
\hline $\mathrm{H}$ & -2.06670500 & -3.92102800 & -2.33721100 \\
\hline $\mathrm{H}$ & -0.31430700 & -5.41242600 & -3.27657800 \\
\hline $\mathrm{H}$ & 1. 78735100 & -5.83404000 & -1.99203000 \\
\hline $\mathrm{H}$ & 2. 28815300 & -5.80926600 & 1. 05858800 \\
\hline $\mathrm{H}$ & 3. 30706800 & -4.66479800 & 0.17558700 \\
\hline $\mathrm{H}$ & 2. 61215400 & -2.80984700 & 1. 49194200 \\
\hline $\mathrm{H}$ & 2. 35792400 & -4.04022500 & 2. 73605800 \\
\hline $\mathrm{H}$ & -1.31395500 & -4.48641200 & 2. 16175100 \\
\hline $\mathrm{H}$ & 0.14611100 & -5.45473700 & 2. 44696500 \\
\hline $\mathrm{H}$ & -1.14660600 & -4.17261300 & 4. 54040200 \\
\hline $\mathrm{H}$ & 0.62232900 & -4.20344100 & 4. 51381000 \\
\hline $\mathrm{H}$ & -0.76439900 & -1.73671100 & 5. 68503300 \\
\hline $\mathrm{H}$ & -0.44706400 & 0.69386100 & 5. 10419700 \\
\hline $\mathrm{H}$ & 0.26956400 & 1. 31459200 & 2. 86805500 \\
\hline $\mathrm{H}$ & 3. 06463700 & -0.16640100 & 2. 24882200 \\
\hline $\mathrm{H}$ & 6.27926600 & -1.60180400 & -0.21592300 \\
\hline $\mathrm{H}$ & 2. 39848400 & -0.83623000 & -1.93078200 \\
\hline $\mathrm{H}$ & 3. 45712500 & -0.79182500 & -3.86463000 \\
\hline $\mathrm{H}$ & 3. 08223200 & -2.47764700 & -3.44502600 \\
\hline $\mathrm{H}$ & 4. 34410200 & -2.11844800 & -4.64662800 \\
\hline $\mathrm{H}$ & 6.40162700 & -3.21805100 & -1.74294500 \\
\hline $\mathrm{H}$ & 5. 98064200 & -3.50939500 & -3.44337900 \\
\hline $\mathrm{H}$ & 4. 81024400 & -3.89902200 & -2.15748400 \\
\hline $\mathrm{H}$ & 5. 68948700 & 0.25172700 & -3.14171300 \\
\hline $\mathrm{H}$ & 6.51435600 & -1.07013300 & -4.00776600 \\
\hline $\mathrm{H}$ & 6.91634500 & -0.73134600 & -2.30830400 \\
\hline $\mathrm{H}$ & 4. 76045900 & 0.66739700 & 3. 54809200 \\
\hline $\mathrm{H}$ & 5. 84942100 & -0.38586800 & 4. 47414000 \\
\hline $\mathrm{H}$ & 4. 26378800 & -0.99434700 & 3. 95889300 \\
\hline $\mathrm{H}$ & 5. 42858200 & -2.99785400 & 2. 84506500 \\
\hline $\mathrm{H}$ & 6. 97328900 & -2.35172500 & 3. 45703400 \\
\hline
\end{tabular}




\begin{tabular}{|c|c|c|c|}
\hline H & 6. 79214900 & -2.74049300 & 1. 73201800 \\
\hline $\mathrm{H}$ & 6.66415800 & 1.06327900 & 1. 86269200 \\
\hline $\mathrm{H}$ & 7. 53578900 & -0.31422100 & 1. 15137000 \\
\hline $\mathrm{H}$ & 7. 69313300 & 0.03000100 & 2. 88742200 \\
\hline $\mathrm{H}$ & -1.00408700 & 2.21588300 & 1. 24997500 \\
\hline $\mathrm{H}$ & 1. 45321900 & 5. 72416200 & 1. 13387200 \\
\hline H & 3. 11030700 & 1. 91836200 & 0.03453200 \\
\hline $\mathrm{H}$ & 4. 19967300 & 3. 18459800 & -1.49573800 \\
\hline $\mathrm{H}$ & 5. 12692300 & 4. 70272800 & -1.54451800 \\
\hline H & 3. 39373400 & 4. 69597200 & -1.96386600 \\
\hline $\mathrm{H}$ & 4. 77377600 & 6.49605200 & 0.12146300 \\
\hline H & 3.58423000 & 6.36119700 & 1. 43243100 \\
\hline $\mathrm{H}$ & 3. 03949900 & 6.57367900 & -0.25225400 \\
\hline $\mathrm{H}$ & 4. 97945000 & 2. 86775600 & 0.97939800 \\
\hline H & 4. 67133200 & 4. 15851000 & 2. 16521800 \\
\hline $\mathrm{H}$ & 5. 87446600 & 4. 40084600 & 0.87156400 \\
\hline $\mathrm{H}$ & -2.09872500 & 3.35111100 & 2. 93704000 \\
\hline $\mathrm{H}$ & -2.71167300 & 3. 64253400 & 1. 27304000 \\
\hline $\mathrm{H}$ & -3.07933000 & 4. 78730800 & 2.58310800 \\
\hline $\mathrm{H}$ & -0.57404400 & 6.63724200 & 0.50312000 \\
\hline $\mathrm{H}$ & -2.20928000 & 6.67764800 & 1. 19807800 \\
\hline $\mathrm{H}$ & -1.83766000 & 5.52486200 & -0.11170600 \\
\hline $\mathrm{H}$ & 0.30497300 & 6.29594200 & 2. 96230200 \\
\hline $\mathrm{H}$ & -0.27922600 & 4. 90459700 & 3. 90500600 \\
\hline $\mathrm{H}$ & -1.37027100 & 6. 26881700 & 3. 55164400 \\
\hline $\mathrm{C}$ & -4.29651000 & -0.46679500 & -0.93969600 \\
\hline $\mathrm{H}$ & -4.51466000 & -1.42013200 & -1.44493600 \\
\hline $\mathrm{H}$ & -3.79474900 & 0.18522400 & -1.66626900 \\
\hline $\mathrm{C}$ & -5.55943700 & 0.22494800 & -0.46236600 \\
\hline $\mathrm{C}$ & -6.77546600 & -0.49052700 & -0.18215800 \\
\hline $\mathrm{C}$ & -5.52225900 & 1. 60107800 & -0.27970900 \\
\hline $\mathrm{C}$ & -6.92451900 & -1.89886200 & -0.36521600 \\
\hline $\mathrm{C}$ & -7.91929500 & 0.24166200 & 0.29936700 \\
\hline $\mathrm{C}$ & -6.65158000 & 2.31110300 & 0.19289900 \\
\hline $\mathrm{H}$ & -4.60775100 & 2. 15923000 & -0.51398900 \\
\hline $\mathrm{C}$ & -8.11122700 & -2.54503600 & -0.08353900 \\
\hline $\mathrm{H}$ & -6.08701200 & -2.48631800 & -0.74440400 \\
\hline $\mathrm{C}$ & -9.12775200 & -0.45686300 & 0.58086700 \\
\hline $\mathrm{C}$ & -7.82386300 & 1. 64831400 & 0.48112900 \\
\hline $\mathrm{H}$ & -6.58148300 & 3. 39420700 & 0.32088400 \\
\hline $\mathrm{C}$ & -9.22761900 & -1.81901900 & 0.39811000 \\
\hline $\mathrm{H}$ & -8.19149200 & -3.62424400 & -0.23817200 \\
\hline $\mathrm{H}$ & -9.98501400 & 0.11543800 & 0.94669100 \\
\hline $\mathrm{H}$ & -8.69936400 & 2. 19241200 & 0.84650600 \\
\hline . & -10.16332800 & -2.33916000 & 0.61814200 \\
\hline
\end{tabular}




$\begin{array}{lrrr}\mathrm{C} & 1.81524600 & 2.17476900 & -4.15120500 \\ \mathrm{H} & 1.71245000 & 2.53412600 & -5.18824500 \\ \mathrm{H} & 2.71842800 & 1.54878200 & -4.08656100 \\ \mathrm{H} & 1.96436300 & 3.04443900 & -3.49186000 \\ \mathrm{C} & 0.58585000 & 1.36854700 & -3.71341600 \\ \mathrm{O} & 0.86679400 & 0.84277300 & -2.44909300 \\ \mathrm{C} & -0.69845600 & 2.23618500 & -3.77266300 \\ \mathrm{H} & -0.66842700 & 2.82069300 & -4.70784800 \\ \mathrm{H} & -1.58402000 & 1.58571400 & -3.87667000 \\ \mathrm{C} & -0.90020100 & 3.18519700 & -2.57491300 \\ \mathrm{H} & 0.06107200 & 3.34212700 & -2.05647700 \\ \mathrm{H} & -1.25437400 & 4.16913800 & -2.91101200 \\ \mathrm{C} & -1.90364100 & 2.70471100 & -1.52065900 \\ \mathrm{O} & -2.76683800 & 3.48979100 & -1.11220400 \\ \mathrm{O} & -1.81032000 & 1.49589200 & -1.07049400 \\ \mathrm{H} & 0.45008800 & 0.55079300 & -4.46314000 \\ \mathrm{H} & -1.11596400 & -0.99499200 & -2.95259000 \\ \mathrm{H} & -1.60496700 & -0.29640400 & -2.91070200\end{array}$

\section{TSKR1}

Ir

$\mathrm{P}$

0

$\mathrm{N}$

C

C

C

C

C

C

C

C

C

C

C

C

C

C

C

C

C

C

C

C
$-0.59470900$

0. 88211700

$-2.96213300$

$-2.06129300$

$-3.29565100$

$-3.68779500$

$-2.01156300$

$-0.99040600$

$-1.15330300$

$-0.16325800$

1. 00527400

1. 16369100

2. 28852900

2. 01846200

0. 48998200

0. 17632000

$-0.32901900$

$-0.32168600$

$-0.22325500$

$-0.44759900$

$-0.23121500$

0. 18336200

0. 38701200

0. 18341900
$-0.26664900$

0. 06792800

$-2.94094700$

$-1.30724400$

$-0.69846800$

$-1.74267300$

$-2.53611900$

$-3.50176800$

-4. 10559200

$-4.94093300$

$-5.17882800$

$-4.58669400$

$-4.79570500$

$-3.73463300$

$-3.40834600$

$-3.73830600$

$-4.43288500$

$-3.87449300$

$-2.38798100$

$-1.43014100$

$-0.09307900$

0. 25631300

$-0.68220700$

$-2.06283700$
$-1.55603900$

0. 43235700

0. 81021300

$-0.41755500$

0. 14876200

1. 21088000

$-0.04006600$

$-0.50305500$

$-1.75985100$

$-2.27983600$

$-1.54942000$

$-0.29529600$

0. 69033900

1. 77408000

1. 69323200

0. 23552600

2. 54850900

3. 96735700

3. 73660100

4. 72395700

4. 41670900

3. 13151900

2. 09696200

2. 41738000 


\begin{tabular}{|c|c|c|c|}
\hline $\mathrm{C}$ & 2. 61846500 & -0.51533900 & 0.19764700 \\
\hline $\mathrm{C}$ & 3. 49211800 & -0.53127600 & 1. 29825100 \\
\hline $\mathrm{C}$ & 4. 82822700 & -0.92690200 & 1. 15142400 \\
\hline $\mathrm{C}$ & 5. 25163500 & -1.31055100 & -0.13379000 \\
\hline $\mathrm{C}$ & 4. 40925100 & -1.29730100 & -1.25568700 \\
\hline $\mathrm{C}$ & 3. 07907600 & -0.88146400 & -1.07203000 \\
\hline $\mathrm{C}$ & 4. 95850300 & -1.71406100 & -2.63578400 \\
\hline $\mathrm{C}$ & 3. 85736500 & -1.73674000 & -3.71135300 \\
\hline $\mathrm{C}$ & 5.57363700 & -3.12976800 & -2.54747100 \\
\hline $\mathrm{C}$ & 6.04827300 & -0.70740600 & -3.07238200 \\
\hline $\mathrm{C}$ & 5. 82272600 & -0.96445500 & 2. 33122300 \\
\hline $\mathrm{C}$ & 5. 18311500 & -0.48207200 & 3. 64600100 \\
\hline $\mathrm{C}$ & 6.31892600 & -2.41451000 & 2. 53827000 \\
\hline $\mathrm{C}$ & 7. 02943500 & -0.04952900 & 2. 01992900 \\
\hline $\mathrm{C}$ & 1.09574100 & 1. 88867500 & 0.71997300 \\
\hline $\mathrm{C}$ & 0.01139200 & 2. 68090800 & 1. 14895900 \\
\hline $\mathrm{C}$ & 0.15432100 & 4. 05853500 & 1. 34306600 \\
\hline $\mathrm{C}$ & 1. 39910500 & 4. 63974000 & 1. 02705300 \\
\hline $\mathrm{C}$ & 2. 48256200 & 3. 89564200 & 0.55063200 \\
\hline $\mathrm{C}$ & 2.31253100 & 2.50463000 & 0.41332900 \\
\hline $\mathrm{C}$ & 3.84113000 & 4. 53335600 & 0.19028400 \\
\hline $\mathrm{C}$ & 4. 16707100 & 4. 24590900 & -1.29325200 \\
\hline $\mathrm{C}$ & 3. 84344300 & 6.05905400 & 0.39429000 \\
\hline $\mathrm{C}$ & 4. 94649300 & 3. 92698900 & 1. 08578100 \\
\hline $\mathrm{C}$ & -0.98054500 & 4. 94480900 & 1. 89978000 \\
\hline $\mathrm{C}$ & -2.23600800 & 4. 12584400 & 2. 25164100 \\
\hline $\mathrm{C}$ & -1.37965500 & 6.00335900 & 0.84462400 \\
\hline $\mathrm{C}$ & -0.48425800 & 5. 65043200 & 3. 18369900 \\
\hline $\mathrm{H}$ & 0.35747900 & -1.52240700 & -1.70488200 \\
\hline $\mathrm{H}$ & -3.03747400 & 0.27038400 & 0.59087300 \\
\hline $\mathrm{H}$ & -3.33896600 & -1.47277300 & 2. 21953300 \\
\hline $\mathrm{H}$ & -4.75680900 & -1.98342500 & 1. 24244500 \\
\hline $\mathrm{H}$ & -2.05656400 & -3.90076900 & -2.33930500 \\
\hline $\mathrm{H}$ & -0.30273000 & -5.40210800 & -3.26044000 \\
\hline $\mathrm{H}$ & 1. 78206700 & -5.83545300 & -1.95089000 \\
\hline $\mathrm{H}$ & 2. 24403600 & -5.82204400 & 1. 09731000 \\
\hline $\mathrm{H}$ & 3.28355300 & -4.68322100 & 0.23116500 \\
\hline $\mathrm{H}$ & 2. 59145200 & -2.82827700 & 1. 54814300 \\
\hline $\mathrm{H}$ & 2. 31002000 & -4.06145500 & 2. 78365900 \\
\hline $\mathrm{H}$ & -1.36160600 & -4.45895600 & 2. 17053500 \\
\hline $\mathrm{H}$ & 0.08168300 & -5.44995200 & 2. 46268200 \\
\hline $\mathrm{H}$ & -1.21341400 & -4.15609000 & 4. 54986000 \\
\hline $\mathrm{H}$ & 0.55452000 & -4.22426100 & 4. 54302400 \\
\hline $\mathrm{H}$ & -0.76819800 & -1.73672600 & 5. 72339700 \\
\hline $\mathrm{H}$ & -0.37505600 & 0.68810600 & 5. 16716400 \\
\hline
\end{tabular}




\begin{tabular}{|c|c|c|c|}
\hline H & 0.36144100 & 1. 30957000 & 2. 93575100 \\
\hline H & 3. 11334200 & -0.23211200 & 2. 27473700 \\
\hline H & 6.28817100 & -1.62721400 & -0.26509500 \\
\hline $\mathrm{H}$ & 2. 39538100 & -0.79328400 & -1.91449100 \\
\hline H & 3. 39878500 & -0.74736500 & -3.85507500 \\
\hline $\mathrm{H}$ & 3. 05311800 & -2.44474600 & -3.45769300 \\
\hline H & 4. 28719900 & -2.04889600 & -4.67633900 \\
\hline H & 6.41322700 & -3.17768100 & -1.83698600 \\
\hline H & 5.95760300 & -3.44173900 & -3.53244500 \\
\hline H & 4. 82004900 & -3.86808100 & -2.22902200 \\
\hline H & 5. 63412300 & 0.31009300 & -3.15177800 \\
\hline H & 6.45836400 & -0.98540100 & -4.05736200 \\
\hline H & 6.88625400 & -0.67436800 & -2.35859000 \\
\hline H & 4. 82535500 & 0.55663500 & 3. 57203400 \\
\hline H & 5.92763100 & -0.51809300 & 4. 45683900 \\
\hline H & 4. 33447300 & -1.11442800 & 3. 95016900 \\
\hline H & 5. 47978500 & -3.09046800 & 2. 76823000 \\
\hline H & 7. 03261600 & -2.46152300 & 3. 37724500 \\
\hline H & 6.82986000 & -2.80623900 & 1. 64533500 \\
\hline H & 6.70683300 & 0.99313400 & 1. 87198000 \\
\hline H & 7. 56542500 & -0.36675100 & 1. 11246000 \\
\hline $\mathrm{H}$ & 7. 74974100 & -0.06702900 & 2. 85423600 \\
\hline $\mathrm{H}$ & -0.95303700 & 2. 21328500 & 1. 32547000 \\
\hline $\mathrm{H}$ & 1.51245300 & 5. 71465400 & 1. 16029700 \\
\hline $\mathrm{H}$ & 3. 14183000 & 1. 89952700 & 0.05098500 \\
\hline $\mathrm{H}$ & 4. 20965500 & 3. 16706900 & -1.50489800 \\
\hline H & 5. 14360800 & 4. 68078800 & -1.56376100 \\
\hline $\mathrm{H}$ & 3. 40409000 & 4. 68409900 & -1.95572200 \\
\hline $\mathrm{H}$ & 4. 82748200 & 6.46925200 & 0.11732000 \\
\hline $\mathrm{H}$ & 3. 65436500 & 6.33587700 & 1. 44328000 \\
\hline $\mathrm{H}$ & 3. 08904200 & 6.55854600 & -0.23325300 \\
\hline $\mathrm{H}$ & 5.02750000 & 2.83721100 & 0.95454100 \\
\hline $\mathrm{H}$ & 4. 74359300 & 4. 12376400 & 2. 15086600 \\
\hline $\mathrm{H}$ & 5.92716400 & 4. 36685700 & 0.83945200 \\
\hline $\mathrm{H}$ & -2.02484900 & 3. 36103700 & 3. 01732800 \\
\hline $\mathrm{H}$ & -2.65585700 & 3. 63989000 & 1. 35892200 \\
\hline $\mathrm{H}$ & -3.00775800 & 4. 79579800 & 2. 66346700 \\
\hline $\mathrm{H}$ & -0.52323400 & 6.62768000 & 0.54359900 \\
\hline $\mathrm{H}$ & -2.15028400 & 6.67595600 & 1. 25740100 \\
\hline $\mathrm{H}$ & -1.79556300 & 5.51445800 & -0.04903500 \\
\hline $\mathrm{H}$ & 0.38300400 & 6.30173400 & 2. 99495700 \\
\hline $\mathrm{H}$ & -0.19174900 & 4. 91708400 & 3. 95321300 \\
\hline $\mathrm{H}$ & -1.28513500 & 6.28057700 & 3. 60427600 \\
\hline $\mathrm{C}$ & -4.33631700 & -0.45014200 & -0.96479500 \\
\hline & -4.56763600 & -1.40297600 & -1.46448000 \\
\hline
\end{tabular}




\begin{tabular}{|c|c|c|c|}
\hline H & -3.84246100 & 0.19224800 & -1.70584600 \\
\hline $\mathrm{C}$ & -5.58688000 & 0.24900500 & -0.46543800 \\
\hline $\mathrm{C}$ & -6.80690800 & -0.45731500 & -0.17925400 \\
\hline $\mathrm{C}$ & -5.53264300 & 1. 62160400 & -0.26242900 \\
\hline $\mathrm{C}$ & -6.97286000 & -1.86174000 & -0.37712300 \\
\hline $\mathrm{C}$ & -7.93665500 & 0.28031300 & 0.32682700 \\
\hline $\mathrm{C}$ & -6.64821600 & 2. 33736100 & 0.23343500 \\
\hline $\mathrm{H}$ & -4.61353800 & 2. 17232500 & -0.49520900 \\
\hline $\mathrm{C}$ & -8.16213900 & -2.49931600 & -0.08694700 \\
\hline $\mathrm{H}$ & -6.14675000 & -2.45278800 & -0.77507300 \\
\hline $\mathrm{C}$ & -9.14841400 & -0.40930600 & 0.61599600 \\
\hline $\mathrm{C}$ & -7.82422700 & 1. 68327300 & 0.52617800 \\
\hline $\mathrm{H}$ & -6.56447300 & 3. 41756100 & 0.37686700 \\
\hline $\mathrm{C}$ & -9.26454400 & -1.76806000 & 0.41840500 \\
\hline $\mathrm{H}$ & -8.25546900 & -3.57571600 & -0.25331400 \\
\hline $\mathrm{H}$ & -9.99469900 & 0.16711000 & 1. 00043400 \\
\hline $\mathrm{H}$ & -8.68921600 & 2. 23144300 & 0.90999900 \\
\hline $\mathrm{H}$ & -10.20242400 & -2.28143600 & 0.64498400 \\
\hline $\mathrm{C}$ & 1. 78830800 & 2. 21214800 & -4.16803900 \\
\hline $\mathrm{H}$ & 1. 68472600 & 2. 61246800 & -5.18984200 \\
\hline $\mathrm{H}$ & 2. 68443000 & 1. 57362800 & -4.13383700 \\
\hline $\mathrm{H}$ & 1. 95257900 & 3. 05383900 & -3.47671400 \\
\hline $\mathrm{C}$ & 0.55090500 & 1. 40474800 & -3.75671900 \\
\hline 0 & 0.82699000 & 0.83488000 & -2.50922000 \\
\hline $\mathrm{C}$ & -0.72467400 & 2. 28360700 & -3.77499400 \\
\hline $\mathrm{H}$ & -0.69904800 & 2. 90437500 & -4.68668900 \\
\hline $\mathrm{H}$ & -1.61366200 & 1. 64096500 & -3.88931100 \\
\hline $\mathrm{C}$ & -0.89908800 & 3. 19212000 & -2.54149000 \\
\hline $\mathrm{H}$ & 0.07611000 & 3. 34405100 & -2.04806700 \\
\hline $\mathrm{H}$ & -1.27216100 & 4. 18144900 & -2.83864300 \\
\hline $\mathrm{C}$ & -1.86597400 & 2. 68510200 & -1.46794700 \\
\hline 0 & -2.73650100 & 3. 44636900 & -1.03680400 \\
\hline 0 & -1.73611400 & 1. 47901900 & -1.00536600 \\
\hline $\mathrm{H}$ & 0.40435200 & 0.61366200 & -4.53064800 \\
\hline $\mathrm{H}$ & -0.80835800 & -1.22484000 & -2.80373000 \\
\hline . & -1.55970300 & -0.29509100 & -2.87043800 \\
\hline
\end{tabular}

\section{IntLR1}

Ir

$\mathrm{P}$

0

N

C

C
$-0.64731000$
$-0.21927700$
$-1.59586000$
0. 93122900
0. 02338600
0. 46543900
$-2.97580500$
$-2.89089800$
0. 81919400
$-2.09724100$
$-1.26199500$
$-0.43493500$
$-3.32596200$
$-0.65520200$
0. 14379500
$-1.69271900$
1. 21888300 


\begin{tabular}{|c|c|c|c|}
\hline C & -2.03837300 & -2.48716700 & -0.04741400 \\
\hline $\mathrm{C}$ & -1.02674900 & -3.45467800 & -0.52828200 \\
\hline $\mathrm{C}$ & -1.20910100 & -4.02868800 & -1.79783100 \\
\hline $\mathrm{C}$ & -0.23552900 & -4.86418500 & -2.34789600 \\
\hline $\mathrm{C}$ & 0.93668900 & -5.13412000 & -1.63440600 \\
\hline $\mathrm{C}$ & 1. 11393400 & -4.57520100 & -0.36770900 \\
\hline $\mathrm{C}$ & 2. 24206400 & -4.83261100 & 0.60205700 \\
\hline $\mathrm{C}$ & 2.00005800 & -3.79833500 & 1. 71666900 \\
\hline $\mathrm{C}$ & 0.47747400 & -3.44179000 & 1. 65958100 \\
\hline $\mathrm{C}$ & 0.14392900 & -3.72473900 & 0.19493600 \\
\hline $\mathrm{C}$ & -0.35682600 & -4.47050100 & 2. 49477800 \\
\hline $\mathrm{C}$ & -0.32754700 & -3.94924000 & 3. 92823200 \\
\hline $\mathrm{C}$ & -0.18833900 & -2.46017100 & 3. 73725600 \\
\hline $\mathrm{C}$ & -0.36801300 & -1.52104700 & 4. 75135800 \\
\hline $\mathrm{C}$ & -0.10778600 & -0.18436800 & 4. 47507900 \\
\hline $\mathrm{C}$ & 0.30383800 & 0.18500500 & 3. 19438100 \\
\hline $\mathrm{C}$ & 0.46076300 & -0.73294500 & 2. 13333500 \\
\hline $\mathrm{C}$ & 0.21238500 & -2.11166100 & 2. 42288700 \\
\hline $\mathrm{C}$ & 2. 67680800 & -0.53470500 & 0.21756000 \\
\hline $\mathrm{C}$ & 3. 55891600 & -0.60824700 & 1. 30847100 \\
\hline $\mathrm{C}$ & 4. 89608200 & -0.98722800 & 1. 13069700 \\
\hline $\mathrm{C}$ & 5. 31300400 & -1.29784500 & -0.17628700 \\
\hline $\mathrm{C}$ & 4. 46279600 & -1.22797100 & -1.29034100 \\
\hline $\mathrm{C}$ & 3. 13209200 & -0.82766700 & -1.07404200 \\
\hline $\mathrm{C}$ & 5. 00600000 & -1.55674800 & -2.69650100 \\
\hline $\mathrm{C}$ & 3. 88628900 & -1.59721100 & -3.75227100 \\
\hline $\mathrm{C}$ & 5. 70126100 & -2.93725600 & -2.68540900 \\
\hline $\mathrm{C}$ & 6. 02837000 & -0.47086700 & -3.10618500 \\
\hline $\mathrm{C}$ & 5. 89953800 & -1.07906300 & 2. 29969600 \\
\hline $\mathrm{C}$ & 5. 26602800 & -0.67272900 & 3. 64273100 \\
\hline $\mathrm{C}$ & 6. 40920500 & -2.53369400 & 2. 42587200 \\
\hline $\mathrm{C}$ & 7. 09543000 & -0.13748700 & 2. 02845000 \\
\hline $\mathrm{C}$ & 1. 13737500 & 1. 84159000 & 0.77902300 \\
\hline $\mathrm{C}$ & 0.04595300 & 2. 61375500 & 1. 22649100 \\
\hline $\mathrm{C}$ & 0.16831200 & 3. 99155200 & 1. 43263900 \\
\hline $\mathrm{C}$ & 1. 40059700 & 4. 59576900 & 1. 11029600 \\
\hline $\mathrm{C}$ & 2. 49115500 & 3. 87249900 & 0.61805900 \\
\hline $\mathrm{C}$ & 2. 34164300 & 2. 48008000 & 0.46964900 \\
\hline $\mathrm{C}$ & 3.83667500 & 4. 53388200 & 0.25169700 \\
\hline $\mathrm{C}$ & 4. 15675100 & 4. 25887000 & -1.23558200 \\
\hline $\mathrm{C}$ & 3. 81594100 & 6. 05847600 & 0.46301000 \\
\hline $\mathrm{C}$ & 4. 95819300 & 3. 94133200 & 1. 13642200 \\
\hline $\mathrm{C}$ & -0.97389500 & 4. 85377500 & 2. 01198100 \\
\hline $\mathrm{C}$ & -2.21978200 & 4. 01486100 & 2. 35053600 \\
\hline $\mathrm{C}$ & -1.38811200 & 5. 93220900 & 0.98314600 \\
\hline
\end{tabular}




\begin{tabular}{|c|c|c|c|}
\hline $\mathrm{C}$ & -0.47986900 & 5.53617600 & 3. 30929300 \\
\hline $\mathrm{H}$ & 0.37460600 & -1.50992200 & -1.78262600 \\
\hline H & -3.06471800 & 0.31632400 & 0.57792100 \\
\hline $\mathrm{H}$ & -3.34128300 & -1.41267400 & 2. 22101100 \\
\hline $\mathrm{H}$ & -4.76877300 & -1.93712100 & 1. 26504900 \\
\hline $\mathrm{H}$ & -2.11474600 & -3.79700200 & -2.36297300 \\
\hline $\mathrm{H}$ & -0.39112300 & -5.30047200 & -3.33739500 \\
\hline H & 1. 70103000 & -5.79144100 & -2.05798400 \\
\hline $\mathrm{H}$ & 2. 17926100 & -5.86911300 & 0.97972800 \\
\hline $\mathrm{H}$ & 3. 23567800 & -4.72781200 & 0.13814700 \\
\hline $\mathrm{H}$ & 2. 58625200 & -2.89524200 & 1. 51063000 \\
\hline $\mathrm{H}$ & 2. 29459800 & -4.15740600 & 2. 71426700 \\
\hline $\mathrm{H}$ & -1.39228500 & -4.46445200 & 2. 12421400 \\
\hline $\mathrm{H}$ & 0.03089600 & -5.49389200 & 2. 38025200 \\
\hline $\mathrm{H}$ & -1.22444300 & -4.22221800 & 4. 50696600 \\
\hline $\mathrm{H}$ & 0.54065500 & -4.33902200 & 4. 49018100 \\
\hline $\mathrm{H}$ & -0.68702400 & -1.84147800 & 5. 74696600 \\
\hline $\mathrm{H}$ & -0.21429700 & 0.58065500 & 5. 24821700 \\
\hline $\mathrm{H}$ & 0.51550400 & 1. 23666100 & 3. 02174400 \\
\hline $\mathrm{H}$ & 3. 18544200 & -0.36398800 & 2. 30215600 \\
\hline $\mathrm{H}$ & 6. 35041800 & -1.60030900 & -0.33131500 \\
\hline $\mathrm{H}$ & 2. 44742500 & -0.68560500 & -1.91023500 \\
\hline $\mathrm{H}$ & 3. 37180700 & -0.63037000 & -3.85125200 \\
\hline $\mathrm{H}$ & 3. 12789700 & -2.35855300 & -3.51059900 \\
\hline $\mathrm{H}$ & 4. 31195800 & -1.85003600 & -4.73620300 \\
\hline $\mathrm{H}$ & 6.56065900 & -2.96950400 & -1.99848600 \\
\hline $\mathrm{H}$ & 6. 07709300 & -3.18248800 & -3.69208100 \\
\hline $\mathrm{H}$ & 4. 99875400 & -3.73114500 & -2.38423600 \\
\hline $\mathrm{H}$ & 5.55618600 & 0.52387400 & -3.13220400 \\
\hline $\mathrm{H}$ & 6. 43498200 & -0.68078900 & -4.10938800 \\
\hline $\mathrm{H}$ & 6.87551900 & -0.42207000 & -2.40417400 \\
\hline $\mathrm{H}$ & 4. 89691400 & 0.36445900 & 3. 62568000 \\
\hline $\mathrm{H}$ & 6. 01738800 & -0.74306300 & 4. 44495500 \\
\hline $\mathrm{H}$ & 4. 42613400 & -1.32883800 & 3. 91958700 \\
\hline $\mathrm{H}$ & 5.57710700 & -3.22743500 & 2. 62695400 \\
\hline $\mathrm{H}$ & 7. 13023400 & -2.61916400 & 3. 25548400 \\
\hline $\mathrm{H}$ & 6.91501900 & -2.87392100 & 1. 50925900 \\
\hline $\mathrm{H}$ & 6.76174700 & 0.90828700 & 1. 93665600 \\
\hline $\mathrm{H}$ & 7. 62858200 & -0.40159500 & 1. 10240800 \\
\hline $\mathrm{H}$ & 7. 82165400 & -0.19054800 & 2. 85616300 \\
\hline $\mathrm{H}$ & -0.90654800 & 2. 12603700 & 1. 41484500 \\
\hline $\mathrm{H}$ & 1. 49826900 & 5. 67112400 & 1. 25220600 \\
\hline $\mathrm{H}$ & 3. 17882700 & 1. 89129900 & 0.09776900 \\
\hline $\mathrm{H}$ & 4. 21832200 & 3. 18182000 & -1.45181600 \\
\hline $\mathrm{H}$ & 5. 12287500 & 4. 71311500 & -1.51169400 \\
\hline
\end{tabular}




\begin{tabular}{|c|c|c|c|}
\hline H & 3. 38060000 & 4. 68506700 & -1.89061700 \\
\hline $\mathrm{H}$ & 4. 79087300 & 6. 48607300 & 0.18015900 \\
\hline $\mathrm{H}$ & 3. 63088100 & 6.32709700 & 1. 51483500 \\
\hline $\mathrm{H}$ & 3. 04838100 & 6.54861800 & -0.15591700 \\
\hline $\mathrm{H}$ & 5. 05668000 & 2. 85371500 & 0.99892300 \\
\hline $\mathrm{H}$ & 4. 75906500 & 4. 12896900 & 2. 20384900 \\
\hline H & 5. 92965200 & 4. 39909700 & 0.88601200 \\
\hline $\mathrm{H}$ & -1.99923800 & 3. 23839200 & 3. 10146200 \\
\hline $\mathrm{H}$ & -2.63569200 & 3.53975200 & 1. 45027500 \\
\hline H & -2.99853500 & 4. 66838300 & 2. 77542200 \\
\hline $\mathrm{H}$ & -0.54028700 & 6.57394400 & 0.69512300 \\
\hline H & -2.16585000 & 6.58536100 & 1. 41331700 \\
\hline $\mathrm{H}$ & -1.79984100 & 5. 46112500 & 0.07819900 \\
\hline $\mathrm{H}$ & 0.38319000 & 6. 19608900 & 3. 13147200 \\
\hline H & -0.18076100 & 4. 78893400 & 4. 06267400 \\
\hline $\mathrm{H}$ & -1.28374300 & 6. 15242600 & 3. 74465500 \\
\hline $\mathrm{C}$ & -4.38008300 & -0.42145900 & -0.95979500 \\
\hline $\mathrm{H}$ & -4.60858200 & -1.37944300 & -1.45018100 \\
\hline $\mathrm{H}$ & -3.89959000 & 0.21422200 & -1.71533100 \\
\hline $\mathrm{C}$ & -5.63101100 & 0.27057900 & -0.45242300 \\
\hline $\mathrm{C}$ & -6.84818200 & -0.44106600 & -0.16761500 \\
\hline $\mathrm{C}$ & -5.58136200 & 1. 64204900 & -0.24185100 \\
\hline $\mathrm{C}$ & -7.00800100 & -1.84578000 & -0.36822200 \\
\hline $\mathrm{C}$ & -7.98072100 & 0.29086400 & 0.34043600 \\
\hline $\mathrm{C}$ & -6.69873900 & 2. 35250700 & 0.25725300 \\
\hline $\mathrm{H}$ & -4.66243400 & 2. 19392700 & -0.47048400 \\
\hline $\mathrm{C}$ & -8.19497900 & -2.48858500 & -0.08053800 \\
\hline $\mathrm{H}$ & -6.17868200 & -2.43258600 & -0.76559400 \\
\hline $\mathrm{C}$ & -9.19027600 & -0.40404100 & 0.62629900 \\
\hline $\mathrm{C}$ & -7.87311300 & 1.69336600 & 0.54546900 \\
\hline $\mathrm{H}$ & -6.61874900 & 3. 43217600 & 0.40672800 \\
\hline $\mathrm{C}$ & -9.30093200 & -1.76265600 & 0.42480900 \\
\hline $\mathrm{H}$ & -8.28374600 & -3.56506700 & -0.24882800 \\
\hline $\mathrm{H}$ & -10.03914200 & 0.16802100 & 1. 01151600 \\
\hline $\mathrm{H}$ & -8.74014800 & 2. 23718600 & 0.93078300 \\
\hline $\mathrm{H}$ & -10.23715000 & -2.28019600 & 0.64878100 \\
\hline $\mathrm{C}$ & 1. 74874000 & 2. 23710100 & -4.22077000 \\
\hline $\mathrm{H}$ & 1. 63930600 & 2. 66002300 & -5.23285700 \\
\hline $\mathrm{H}$ & 2. 63442700 & 1.58325400 & -4.21274100 \\
\hline $\mathrm{H}$ & 1. 93834000 & 3. 06130300 & -3.51450800 \\
\hline $\mathrm{C}$ & 0.50252200 & 1. 44299300 & -3.80903500 \\
\hline 0 & 0.78028900 & 0.85135600 & -2.57201300 \\
\hline $\mathrm{C}$ & -0.75606800 & 2. 34290500 & -3.78917700 \\
\hline $\mathrm{H}$ & -0.72571900 & 3. 00431000 & -4.67147000 \\
\hline $\mathrm{H}$ & -1.65560500 & 1. 71987600 & -3.91915100 \\
\hline
\end{tabular}




$\begin{array}{lrrr}\mathrm{C} & -0.89559200 & 3.20109700 & -2.51591100 \\ \mathrm{H} & 0.09268800 & 3.33112900 & -2.04318900 \\ \mathrm{H} & -1.27619500 & 4.20111900 & -2.76288300 \\ \mathrm{C} & -1.83476400 & 2.66386800 & -1.43889100 \\ 0 & -2.70712000 & 3.39594000 & -0.97358100 \\ 0 & -1.68963900 & 1.44734900 & -0.98113700 \\ \mathrm{H} & 0.33104200 & 0.66812400 & -4.59260000 \\ \mathrm{H} & -0.26588600 & -1.53510000 & -2.49677400 \\ \mathrm{H} & -1.60763700 & -0.24569900 & -2.86428600\end{array}$

\section{TSLR1}

Ir

$\mathrm{P}$

0

$\mathrm{N}$

C

C

C

C

C

C

C

C

C

C

C

C

C

C

C

C

C

C

C

C

C

C

C

C

C

C

C

C

C

$\begin{array}{rrr}-0.71250400 & -0.17976700 & -1.57633900 \\ 0.92574700 & 0.01635400 & 0.43180700 \\ -2.92226700 & -2.93976100 & 0.75803500 \\ -2.07730700 & -1.24593600 & -0.42460900 \\ -3.32748300 & -0.69173200 & 0.16724300 \\ -3.66681300 & -1.77234600 & 1.20824900 \\ -1.98889400 & -2.48606700 & -0.08765000 \\ -0.95654100 & -3.41762400 & -0.59288000 \\ -1.11692100 & -3.95926000 & -1.87915700 \\ -0.13351800 & -4.78098500 & -2.43125800 \\ 1.02804100 & -5.06588200 & -1.70573600 \\ 1.18569900 & -4.53366000 & -0.42524000 \\ 2.30274500 & -4.80146500 & 0.55516900 \\ 2.04111700 & -3.78520300 & 1.68263900 \\ 0.51586200 & -3.44119700 & 1.61318700 \\ 0.20335700 & -3.70125400 & 0.14099800 \\ -0.31393000 & -4.48754900 & 2.43031700 \\ -0.30632000 & -3.97691200 & 3.86866500 \\ -0.18638300 & -2.48424100 & 3.69066600 \\ -0.39286900 & -1.55255900 & 4.70647600 \\ -0.15246600 & -0.21023800 & 4.43759700 \\ 0.26550700 & 0.17215900 & 3.16254700 \\ 0.44974400 & -0.73880900 & 2.10035600 \\ 0.22388700 & -2.12197500 & 2.38314700 \\ 2.68543500 & -0.53494800 & 0.23843200 \\ 3.54802600 & -0.53933400 & 1.34779400 \\ 4.89309000 & -0.90938500 & 1.21810100 \\ 5.34392500 & -1.27676300 & -0.06272700 \\ 4.51616400 & -1.27770600 & -1.19508500 \\ 3.17431500 & -0.89580900 & -1.02213000 \\ 5.09344500 & -1.67265100 & -2.57023400 \\ 4.01239200 & -1.69894100 & -3.66614200 \\ 5.72658400 & -3.08061400 & -2.48548000\end{array}$




\begin{tabular}{|c|c|c|c|}
\hline $\mathrm{C}$ & 6.17605400 & -0.64668900 & -2.97890900 \\
\hline $\mathrm{C}$ & 5.87069600 & -0.93408800 & 2. 41216400 \\
\hline $\mathrm{C}$ & 5. 20289800 & -0.47103900 & 3. 71982300 \\
\hline $\mathrm{C}$ & 6.39030700 & -2.37595800 & 2. 61895700 \\
\hline $\mathrm{C}$ & 7. 06459300 & 0.00485500 & 2. 12395300 \\
\hline $\mathrm{C}$ & 1. 14291000 & 1. 83723800 & 0.75024700 \\
\hline $\mathrm{C}$ & 0.05775400 & 2. 61341100 & 1. 20777300 \\
\hline $\mathrm{C}$ & 0.18111200 & 3. 99301600 & 1. 40267500 \\
\hline $\mathrm{C}$ & 1. 40633700 & 4. 59770400 & 1. 05571100 \\
\hline $\mathrm{C}$ & 2. 48893500 & 3. 87239500 & 0.54939000 \\
\hline $\mathrm{C}$ & 2.34126700 & 2.47789000 & 0.41845000 \\
\hline $\mathrm{C}$ & 3. 82202400 & 4. 53487200 & 0.14200100 \\
\hline $\mathrm{C}$ & 4. 08922900 & 4. 26397900 & -1.35689600 \\
\hline $\mathrm{C}$ & 3. 80857000 & 6. 05872900 & 0.35878200 \\
\hline $\mathrm{C}$ & 4. 97457600 & 3. 93998500 & 0.98383200 \\
\hline $\mathrm{C}$ & -0.95193200 & 4. 85682500 & 1. 99763600 \\
\hline $\mathrm{C}$ & -2.20064000 & 4. 02307400 & 2. 33858800 \\
\hline $\mathrm{C}$ & -1.36836100 & 5. 94812900 & 0.98332600 \\
\hline $\mathrm{C}$ & -0.44133200 & 5.52316300 & 3. 29703500 \\
\hline $\mathrm{H}$ & 0.49202800 & -0.67939400 & -2.67496200 \\
\hline $\mathrm{H}$ & -3.09677200 & 0.27359300 & 0.62977400 \\
\hline $\mathrm{H}$ & -3.29950200 & -1.51954500 & 2. 21495300 \\
\hline $\mathrm{H}$ & -4.72869400 & -2.04012600 & 1. 25860700 \\
\hline $\mathrm{H}$ & -2.01177100 & -3.71274500 & -2.45453200 \\
\hline $\mathrm{H}$ & -0.27215400 & -5.19391500 & -3.43324100 \\
\hline $\mathrm{H}$ & 1. 79945400 & -5.71326900 & -2.13206700 \\
\hline $\mathrm{H}$ & 2. 24123500 & -5.84374300 & 0.91701400 \\
\hline $\mathrm{H}$ & 3. 30193200 & -4.68408600 & 0.10617800 \\
\hline $\mathrm{H}$ & 2. 62246800 & -2.87489100 & 1. 49640500 \\
\hline $\mathrm{H}$ & 2.32749700 & -4.15719000 & 2. 67794600 \\
\hline $\mathrm{H}$ & -1.34532800 & -4.49306800 & 2. 04890100 \\
\hline $\mathrm{H}$ & 0.08920200 & -5.50459200 & 2. 31235200 \\
\hline $\mathrm{H}$ & -1.20486800 & -4.26711800 & 4. 43641800 \\
\hline $\mathrm{H}$ & 0.56169800 & -4.35909300 & 4. 43614000 \\
\hline $\mathrm{H}$ & -0.71762800 & -1.88177100 & 5. 69736900 \\
\hline $\mathrm{H}$ & -0.28041800 & 0.54953200 & 5. 21274100 \\
\hline $\mathrm{H}$ & 0.46114100 & 1. 22805400 & 2. 99684900 \\
\hline $\mathrm{H}$ & 3. 15092800 & -0.24845900 & 2. 31926100 \\
\hline $\mathrm{H}$ & 6. 38900400 & -1.56923300 & -0.18115700 \\
\hline $\mathrm{H}$ & 2. 50555000 & -0.84563000 & -1.87678800 \\
\hline $\mathrm{H}$ & 3. 53495600 & -0.71664900 & -3.80088400 \\
\hline $\mathrm{H}$ & 3. 22097200 & -2.43137900 & -3.44208800 \\
\hline $\mathrm{H}$ & 4. 46590300 & -1.98353800 & -4.62867500 \\
\hline $\mathrm{H}$ & 6. 55405700 & -3.12236100 & -1.76073100 \\
\hline $\mathrm{H}$ & 6. 13159100 & -3.37838200 & -3.46643200 \\
\hline
\end{tabular}




\begin{tabular}{|c|c|c|c|}
\hline H & 4. 97858400 & -3.83221300 & -2.18559600 \\
\hline $\mathrm{H}$ & 5. 74924000 & 0.36581000 & -3.05600700 \\
\hline $\mathrm{H}$ & 6.60674200 & -0.90968300 & -3.95917800 \\
\hline $\mathrm{H}$ & 7. 00109400 & -0.60865300 & -2.25072300 \\
\hline H & 4. 82796200 & 0.56160300 & 3. 64674800 \\
\hline $\mathrm{H}$ & 5. 93582400 & -0.49911400 & 4. 54148400 \\
\hline $\mathrm{H}$ & 4. 36097500 & -1.11990000 & 4. 00714400 \\
\hline $\mathrm{H}$ & 5.56042100 & -3.06785800 & 2. 83454400 \\
\hline $\mathrm{H}$ & 7. 09329000 & -2.41394900 & 3. 46737300 \\
\hline H & 6.92024100 & -2.75428100 & 1. 73129100 \\
\hline $\mathrm{H}$ & 6.72478000 & 1.04196900 & 1. 97522900 \\
\hline H & 7. 62204400 & -0.29785700 & 1. 22434300 \\
\hline H & 7. 77131300 & -0.00270100 & 2. 96994900 \\
\hline $\mathrm{H}$ & -0.89255500 & 2. 12657000 & 1. 40824000 \\
\hline H & 1.50381200 & 5. 67434600 & 1. 18742800 \\
\hline $\mathrm{H}$ & 3. 17623300 & 1. 88880600 & 0.04162000 \\
\hline $\mathrm{H}$ & 4. 14750400 & 3. 18707100 & -1.57572900 \\
\hline $\mathrm{H}$ & 5. 04272900 & 4. 72229800 & -1.66806600 \\
\hline $\mathrm{H}$ & 3. 28765600 & 4. 68843700 & -1.98211200 \\
\hline $\mathrm{H}$ & 4. 77556900 & 6. 48677100 & 0.05046100 \\
\hline $\mathrm{H}$ & 3. 65286400 & 6.32308200 & 1. 41642700 \\
\hline $\mathrm{H}$ & 3. 02455300 & 6.55216600 & -0.23638900 \\
\hline $\mathrm{H}$ & 5. 06869600 & 2. 85287700 & 0.83998300 \\
\hline $\mathrm{H}$ & 4. 81445600 & 4. 12476900 & 2. 05826700 \\
\hline $\mathrm{H}$ & 5. 93614500 & 4. 39881700 & 0.69949700 \\
\hline $\mathrm{H}$ & -1.98017900 & 3. 23843400 & 3. 08087800 \\
\hline $\mathrm{H}$ & -2.62818800 & 3.55914600 & 1. 43784700 \\
\hline $\mathrm{H}$ & -2.97121500 & 4. 67825800 & 2. 77558700 \\
\hline $\mathrm{H}$ & -0.52049000 & 6.59070600 & 0.69727700 \\
\hline $\mathrm{H}$ & -2.14048200 & 6.59908600 & 1. 42659300 \\
\hline $\mathrm{H}$ & -1.78862500 & 5. 48901600 & 0.07608800 \\
\hline $\mathrm{H}$ & 0.42678400 & 6.17599600 & 3. 11724900 \\
\hline $\mathrm{H}$ & -0.14306800 & 4. 76657400 & 4. 04137500 \\
\hline $\mathrm{H}$ & -1.23571700 & 6. 14314900 & 3. 74438700 \\
\hline $\mathrm{C}$ & -4.38879700 & -0.46101000 & -0.93001900 \\
\hline $\mathrm{H}$ & -4.61906300 & -1.41942400 & -1.41894600 \\
\hline $\mathrm{H}$ & -3.91504300 & 0.17621400 & -1.68770300 \\
\hline $\mathrm{C}$ & -5.63551600 & 0.23081400 & -0.41218600 \\
\hline $\mathrm{C}$ & -6.84802400 & -0.48074700 & -0.10835600 \\
\hline $\mathrm{C}$ & -5.58546300 & 1. 60379400 & -0.20986700 \\
\hline $\mathrm{C}$ & -7.00975200 & -1.88596600 & -0.30411700 \\
\hline $\mathrm{C}$ & -7.97408000 & 0.25159400 & 0.41337000 \\
\hline $\mathrm{C}$ & -6.69687900 & 2. 31478900 & 0.30148000 \\
\hline $\mathrm{H}$ & -4.67186300 & 2. 15757800 & -0.45455900 \\
\hline$C$ & -8.19166600 & -2.52925100 & 0.00273500 \\
\hline
\end{tabular}




$\begin{array}{lrrr}\mathrm{H} & -6.18665600 & -2.47233800 & -0.71490100 \\ \mathrm{C} & -9.17831500 & -0.44395200 & 0.71956200 \\ \mathrm{C} & -7.86562500 & 1.65509200 & 0.61075100 \\ \mathrm{H} & -6.61641900 & 3.39536500 & 0.44397700 \\ \mathrm{C} & -9.29039300 & -1.80325800 & 0.52362300 \\ \mathrm{H} & -8.28221300 & -3.60604000 & -0.16264200 \\ \mathrm{H} & -10.02204500 & 0.12828400 & 1.11568400 \\ \mathrm{H} & -8.72766200 & 2.19932300 & 1.00659200 \\ \mathrm{H} & -10.22251500 & -2.32121500 & 0.76323500 \\ \mathrm{C} & 1.60024400 & 2.15393600 & -4.48224400 \\ \mathrm{H} & 1.39815800 & 2.63931600 & -5.45070900 \\ \mathrm{H} & 2.44796800 & 1.46349900 & -4.61225500 \\ \mathrm{H} & 1.90420300 & 2.92805000 & -3.75954800 \\ \mathrm{C} & 0.36951500 & 1.39644800 & -3.98100300 \\ \mathrm{O} & 0.76753900 & 0.70413100 & -2.80323300 \\ \mathrm{C} & -0.84167500 & 2.32363500 & -3.74642000 \\ \mathrm{H} & -0.89341800 & 3.02993400 & -4.59133300 \\ \mathrm{H} & -1.76217600 & 1.72343000 & -3.80633600 \\ \mathrm{C} & -0.81457400 & 3.11016400 & -2.41241100 \\ \mathrm{H} & 0.17403300 & 3.00626600 & -1.93564500 \\ \mathrm{H} & -0.98635700 & 4.17923600 & -2.59201100 \\ \mathrm{C} & -1.86811900 & 2.67655500 & -1.38761900 \\ \mathrm{O} & -2.72204200 & 3.47681300 & -1.00784700 \\ \mathrm{O} & -1.82247600 & 1.46459400 & -0.90860900 \\ \mathrm{H} & 0.07327900 & 0.66293800 & -4.75968600 \\ \mathrm{H} & 0.10733200 & -1.51488700 & -2.19415400 \\ \mathrm{H} & -1.70773200 & -0.28705300 & -2.81352300\end{array}$

\section{InKR2}

$\begin{array}{rrrr}\text { Ir } & 0.55905300 & 0.03361500 & 1.36474900 \\ \mathrm{P} & -1.04113900 & 0.16718400 & -0.39084100 \\ \mathrm{O} & 2.75652100 & -2.73742600 & -1.02687600 \\ \mathrm{~N} & 1.95603100 & -1.05393500 & 0.19279700 \\ \mathrm{C} & 3.20568000 & -0.51383500 & -0.41658600 \\ \mathrm{C} & 3.46546800 & -1.55888600 & -1.51047100 \\ \mathrm{C} & 1.85592000 & -2.29211600 & -0.14385100 \\ \mathrm{C} & 0.84797000 & -3.24479600 & 0.37085600 \\ \mathrm{C} & 1.02409100 & -3.79548800 & 1.65102900 \\ \mathrm{C} & 0.07473600 & -4.67325300 & 2.17623400 \\ \mathrm{C} & -1.06712000 & -5.00056000 & 1.43563000 \\ \mathrm{C} & -1.24854900 & -4.44043300 & 0.17051700 \\ \mathrm{C} & -2.35968500 & -4.70706200 & -0.81766400 \\ \mathrm{C} & -2.15295800 & -3.61882800 & -1.88935700 \\ \mathrm{C} & -0.63814800 & -3.22832400 & -1.81911300\end{array}$




\begin{tabular}{|c|c|c|c|}
\hline $\mathrm{C}$ & -0.29914600 & -3.55544200 & -0.36897300 \\
\hline $\mathrm{C}$ & 0.20020200 & -4.19463900 & -2.72038200 \\
\hline $\mathrm{C}$ & 0.14336300 & -3.58623800 & -4.11967000 \\
\hline $\mathrm{C}$ & 0.00237900 & -2.11184800 & -3.83493300 \\
\hline $\mathrm{C}$ & 0.18617400 & -1.10622700 & -4.78249700 \\
\hline $\mathrm{C}$ & -0.04661200 & 0.21208900 & -4.40977500 \\
\hline $\mathrm{C}$ & -0.43571100 & 0.49824400 & -3.10087400 \\
\hline $\mathrm{C}$ & -0.60397800 & -0.49144400 & -2.10922200 \\
\hline $\mathrm{C}$ & -0.38721200 & -1.85085700 & -2.49756900 \\
\hline $\mathrm{C}$ & -2.71499000 & -0.56727200 & -0.08207400 \\
\hline $\mathrm{C}$ & -3.68777400 & -0.49399800 & -1.09457700 \\
\hline $\mathrm{C}$ & -4.98449000 & -0.98389800 & -0.89149900 \\
\hline $\mathrm{C}$ & -5.27688400 & -1.54241400 & 0.36619400 \\
\hline $\mathrm{C}$ & -4.33618200 & -1.63025200 & 1. 40273100 \\
\hline $\mathrm{C}$ & -3.04328000 & -1.13705500 & 1. 15262900 \\
\hline $\mathrm{C}$ & -4.73991100 & -2.24538700 & 2. 75904200 \\
\hline $\mathrm{C}$ & -3.56875400 & -2.26701800 & 3. 75795300 \\
\hline $\mathrm{C}$ & -5.21911600 & -3.69993400 & 2. 54617400 \\
\hline $\mathrm{C}$ & -5.88817600 & -1.41278800 & 3. 37557000 \\
\hline $\mathrm{C}$ & -6.07036400 & -0.94852100 & -1.98753200 \\
\hline $\mathrm{C}$ & -5.58589600 & -0.23461200 & -3.26263300 \\
\hline $\mathrm{C}$ & -6.45844600 & -2.39834500 & -2.36100200 \\
\hline $\mathrm{C}$ & -7.31940500 & -0.20254500 & -1.46451800 \\
\hline $\mathrm{C}$ & -1.44716800 & 1. 97543000 & -0.59794800 \\
\hline $\mathrm{C}$ & -0.43441700 & 2. 87191100 & -0.99983400 \\
\hline $\mathrm{C}$ & -0.67314500 & 4. 24768800 & -1.07928700 \\
\hline $\mathrm{C}$ & -1.93737400 & 4. 71924300 & -0.67179000 \\
\hline $\mathrm{C}$ & -2.95318600 & 3. 86924600 & -0.22512700 \\
\hline $\mathrm{C}$ & -2.69225300 & 2. 48507600 & -0.21225400 \\
\hline $\mathrm{C}$ & -4.33343100 & 4. 38583700 & 0.23324100 \\
\hline $\mathrm{C}$ & -4.60351400 & 3. 92351300 & 1. 68410400 \\
\hline $\mathrm{C}$ & -4.42409700 & 5. 92230300 & 0.19775100 \\
\hline $\mathrm{C}$ & -5.42809500 & 3. 81711700 & -0.69904000 \\
\hline $\mathrm{C}$ & 0.39089800 & 5. 24720100 & -1.58150200 \\
\hline $\mathrm{C}$ & 1. 63618600 & 4. 53798900 & -2.14738000 \\
\hline $\mathrm{C}$ & 0.84233200 & 6. 14975300 & -0.40891900 \\
\hline $\mathrm{C}$ & -0.21884600 & 6. 12189400 & -2.70220700 \\
\hline $\mathrm{H}$ & -0.08891500 & -1.32552900 & 1. 83046200 \\
\hline $\mathrm{H}$ & 2. 99745600 & 0.48281900 & -0.82069900 \\
\hline $\mathrm{H}$ & 3. 01731900 & -1.28527800 & -2.47877300 \\
\hline $\mathrm{H}$ & 4. 52001900 & -1.82392700 & -1.64734200 \\
\hline $\mathrm{H}$ & 1. 89707200 & -3.50908100 & 2. 24071100 \\
\hline $\mathrm{H}$ & 0.22396200 & -5.09709600 & 3. 17210300 \\
\hline $\mathrm{H}$ & -1.80760200 & -5.69457700 & 1. 84319800 \\
\hline $\mathrm{H}$ & -2.25249700 & -5.72299000 & -1.23903200 \\
\hline
\end{tabular}




\begin{tabular}{|c|c|c|c|}
\hline H & -3.36015000 & -4.66165100 & -0.35934400 \\
\hline $\mathrm{H}$ & -2.76403900 & -2.74312100 & -1.64360800 \\
\hline $\mathrm{H}$ & -2.44141600 & -3.94515300 & -2.89998300 \\
\hline $\mathrm{H}$ & 1. 23963500 & -4.20026400 & -2.36270600 \\
\hline $\mathrm{H}$ & -0.17645100 & -5.22691700 & -2.66512500 \\
\hline $\mathrm{H}$ & 1. 03085400 & -3.81684300 & -4.73037300 \\
\hline H & -0.73295700 & -3.94623900 & -4.68887700 \\
\hline $\mathrm{H}$ & 0.49394300 & -1.35983200 & -5.80064200 \\
\hline $\mathrm{H}$ & 0.07066300 & 1. 02829800 & -5.12679600 \\
\hline H & -0.61898300 & 1. 53956300 & -2.85201100 \\
\hline $\mathrm{H}$ & -3.41753900 & -0.04563000 & -2.04992000 \\
\hline H & -6.28368600 & -1.92589200 & 0.54301600 \\
\hline $\mathrm{H}$ & -2.27529000 & -1.20213700 & 1. 91951300 \\
\hline H & -3.20346600 & -1.25385700 & 3. 98666200 \\
\hline H & -2.71887100 & -2.85878300 & 3. 38492000 \\
\hline $\mathrm{H}$ & -3.89766600 & -2.71852100 & 4. 70705000 \\
\hline H & -6.08635300 & -3.75633900 & 1. 87037400 \\
\hline $\mathrm{H}$ & -5.51808600 & -4.15024200 & 3. 50679700 \\
\hline $\mathrm{H}$ & -4.41599400 & -4.32000900 & 2. 11728300 \\
\hline $\mathrm{H}$ & -5.57621400 & -0.36892400 & 3. 53858100 \\
\hline $\mathrm{H}$ & -6.18714100 & -1.83352400 & 4. 34956400 \\
\hline $\mathrm{H}$ & -6.78049200 & -1.39940000 & 2. 73112600 \\
\hline $\mathrm{H}$ & -5.30018800 & 0.81019000 & -3.06495200 \\
\hline $\mathrm{H}$ & -6.39401100 & -0.22123500 & -4.01061200 \\
\hline $\mathrm{H}$ & -4.72368300 & -0.74384700 & -3.72050100 \\
\hline $\mathrm{H}$ & -5.58870000 & -2.95195300 & -2.74930100 \\
\hline $\mathrm{H}$ & -7.23783800 & -2.39933500 & -3.14065100 \\
\hline $\mathrm{H}$ & -6.85132100 & -2.95403800 & -1.49565700 \\
\hline $\mathrm{H}$ & -7.07483500 & 0.83514600 & -1.18799500 \\
\hline $\mathrm{H}$ & -7.75641900 & -0.69140000 & -0.58047500 \\
\hline $\mathrm{H}$ & -8.09919900 & -0.17079500 & -2.24277500 \\
\hline $\mathrm{H}$ & 0.55608400 & 2. 48508700 & -1.22243400 \\
\hline $\mathrm{H}$ & -2.12088100 & 5. 79204000 & -0.70359100 \\
\hline $\mathrm{H}$ & -3.47437300 & 1. 80335100 & 0.11725600 \\
\hline $\mathrm{H}$ & -4.60483800 & 2.82660000 & 1. 77498800 \\
\hline $\mathrm{H}$ & -5.58681000 & 4. 28566500 & 2. 02727500 \\
\hline $\mathrm{H}$ & -3.83803300 & 4. 31642100 & 2. 37207800 \\
\hline $\mathrm{H}$ & -5.41960400 & 6.24472200 & 0.54157600 \\
\hline $\mathrm{H}$ & -4.28255900 & 6.32063700 & -0.81900800 \\
\hline $\mathrm{H}$ & -3.67780600 & 6. 39271700 & 0.85669800 \\
\hline $\mathrm{H}$ & -5.44269100 & 2. 71657700 & -0.68719000 \\
\hline $\mathrm{H}$ & -5.26538700 & 4. 13964800 & -1.73993000 \\
\hline $\mathrm{H}$ & -6.42520300 & 4. 16859600 & -0.38579400 \\
\hline $\mathrm{H}$ & 1. 37618100 & 3. 86197700 & -2.97883900 \\
\hline . & 2. 17435500 & 3. 97091500 & -1.37319500 \\
\hline
\end{tabular}




\begin{tabular}{|c|c|c|c|}
\hline H & 2. 33509600 & 5. 29194800 & -2.54382900 \\
\hline $\mathrm{H}$ & -0.00822800 & 6.66903100 & 0.06108100 \\
\hline $\mathrm{H}$ & 1. 54537400 & 6. 91819000 & -0.77128400 \\
\hline $\mathrm{H}$ & 1. 36467500 & 5. 55712700 & 0.35661800 \\
\hline $\mathrm{H}$ & -1.06997000 & 6.72555600 & -2.35104800 \\
\hline $\mathrm{H}$ & -0.57074600 & 5. 50316600 & -3.54399500 \\
\hline H & 0.53997200 & 6. 82050200 & -3.09039200 \\
\hline $\mathrm{C}$ & 4. 31873500 & -0.40470900 & 0.64777400 \\
\hline $\mathrm{H}$ & 4. 47786100 & -1.39497300 & 1. 09782100 \\
\hline H & 3. 91976600 & 0.22019200 & 1. 45655900 \\
\hline $\mathrm{C}$ & 5. 60585400 & 0.17811500 & 0.09718500 \\
\hline $\mathrm{C}$ & 6.74538500 & -0.64077700 & -0.22475900 \\
\hline $\mathrm{C}$ & 5. 67701200 & 1. 54827400 & -0.11625500 \\
\hline $\mathrm{C}$ & 6. 77960400 & -2.05580000 & -0.03426200 \\
\hline $\mathrm{C}$ & 7. 92640600 & -0.01484500 & -0.76352100 \\
\hline $\mathrm{C}$ & 6.84049100 & 2. 15402400 & -0.64754100 \\
\hline $\mathrm{H}$ & 4. 82164900 & 2. 18524900 & 0.13455600 \\
\hline $\mathrm{C}$ & 7. 89378300 & -2.80416800 & -0.35613100 \\
\hline $\mathrm{H}$ & 5.90878700 & -2.56551800 & 0.38006100 \\
\hline $\mathrm{C}$ & 9. 05877500 & -0.81702900 & -1.08301500 \\
\hline $\mathrm{C}$ & 7. 94235300 & 1. 39191400 & -0.96616100 \\
\hline $\mathrm{H}$ & 6.85433000 & 3. 23670200 & -0.79664100 \\
\hline $\mathrm{C}$ & 9.04902300 & -2.18098400 & -0.88763900 \\
\hline $\mathrm{H}$ & 7. 88585600 & -3.88541600 & -0.19500200 \\
\hline $\mathrm{H}$ & 9. 94622000 & -0.32378100 & -1.48987400 \\
\hline $\mathrm{H}$ & 8.84485000 & 1. 85507800 & -1.37492400 \\
\hline $\mathrm{H}$ & 9.92740000 & -2.78157900 & -1.13753500 \\
\hline $\mathrm{C}$ & 0.78365700 & 0.60387500 & 4. 87448600 \\
\hline $\mathrm{H}$ & 0.99232800 & 0.99791000 & 5.88280800 \\
\hline $\mathrm{H}$ & 0.40941500 & -0.42662600 & 4. 97980600 \\
\hline $\mathrm{H}$ & -0.02553400 & 1. 22169200 & 4. 44990900 \\
\hline $\mathrm{C}$ & 2. 05858800 & 0.61209100 & 4. 01015200 \\
\hline 0 & 1. 97714600 & -0.15174100 & 2. 84562900 \\
\hline $\mathrm{C}$ & 2.59692600 & 2. 04540600 & 3. 78266600 \\
\hline $\mathrm{H}$ & 2. 62749500 & 2. 55613600 & 4. 76174800 \\
\hline $\mathrm{H}$ & 3. 64585100 & 1. 97708600 & 3. 44782300 \\
\hline $\mathrm{C}$ & 1. 83301000 & 2. 94011600 & 2. 78356800 \\
\hline $\mathrm{H}$ & 0.74238300 & 2. 81787500 & 2. 89918200 \\
\hline $\mathrm{H}$ & 2. 06497800 & 3. 99108800 & 3. 00589500 \\
\hline $\mathrm{C}$ & 2.24527600 & 2. 71995300 & 1. 32170800 \\
\hline 0 & 3. 05960800 & 3. 49710300 & 0.81559200 \\
\hline 0 & 1. 74022500 & 1. 72606600 & 0.66197700 \\
\hline $\mathrm{H}$ & 2. 83129700 & 0.10941300 & 4. 63253400 \\
\hline $\mathrm{H}$ & -0.58516300 & 0.30264500 & 2. 48790300 \\
\hline $\mathrm{H}$ & -0.35725600 & 1. 18006800 & 2. 10683900 \\
\hline
\end{tabular}




\section{TSKR2}

Ir

$\mathrm{P}$

0

$\mathrm{N}$

C

C

C

C

C

C

C

C

C

C

C

C

C

C

C

C

C

C

C

C

C

C

C

C

C

C

C

C

C

C

C

C

C

C

C

C

C

C

C

C
$-0.55176200$

1. 05325000

$-2.79658200$

$-1.99016300$

$-3.23707900$

$-3.51041200$

$-1.89313200$

$-0.88721100$

$-1.07420500$

$-0.12883400$

1. 01900600

1. 21048600

2. 32691800

2. 13053300

0. 61741200

0. 26701000

$-0.22264400$

$-0.15536200$

$-0.00108300$

$-0.16467700$

0. 08591100

0. 47043500

0. 61507500

0.38227600

2. 72475500

3. 69363500

4. 98880700

5. 28406400

4. 34773600

3. 05584100

4. 75610500

3. 58885900

5. 23414800

5. 90698900

6. 07031600

5. 58155600

6. 45592000

7. 32171600

1. 46543900

0. 46882500

0. 71537900

1. 96842000

2. 96657600

2. 70013300
0. 04885300

$-1.36952900$

0. 15961600

0. 38900400

$-2.74581600$

$-1.05607700$

1. 02559300

$-0.51686800$

$-1.57059500$

$-0.18532100$

$-2.29427100$

0. 42517400

$-3.24668600$

$-3.79566900$

$-4.67106300$

$-5.00039500$

$-4.44405800$

$-4.71912300$

$-3.63635000$

$-3.23731800$

$-3.55850400$

$-4.20121500$

$-3.59857900$

-2. 12448000

$-1.12229600$

0. 19448200

0. 48312000

$-0.50195200$

$-1.86072400$

$-0.58121800$

$-0.50596900$

$-1.00293500$

$-1.57091800$

$-1.66033500$

$-1.15980200$

$-2.28255500$

$-2.30854700$

$-3.73618100$

$-1.45297800$

$-0.96378100$

$-0.24347700$

-2. 41200900

-0. 22107100

1. 96601100

2. 86592500

4. 24049300

4. 70798800

3. 85454200

2. 47144700
1. 50886900

0. 14686300

$-0.37475500$

$-1.65430500$

-2. 19083900

$-1.46075800$

$-0.19536800$

0. 78435000

1. 86317800

1. 80287900

0. 35391500

2. 70508000

4. 10626300

3. 82711600

4. 78207900

4. 41628600

3. 10648700

2. 10665200

2. 48857000

0. 08316100

1. 09925000

0. 90515600

$-0.34763100$

$-1.38794800$

$-1.14664200$

$-2.73969900$

$-3.74307500$

$-2.51856500$

$-3.35559600$

2. 00525900

3. 27513100

2. 38718200

1. 48321700

0. 59796400

1. 03066400

1. 10524300

0. 66036200

0. 18147500

0. 17573200 


\begin{tabular}{|c|c|c|c|}
\hline $\mathrm{C}$ & 4. 33198100 & 4. 36597300 & -0.32434100 \\
\hline $\mathrm{C}$ & 4. 54270200 & 3. 91154100 & -1.78752400 \\
\hline $\mathrm{C}$ & 4. 43337900 & 5. 90161300 & -0.28250700 \\
\hline $\mathrm{C}$ & 5. 45857600 & 3. 78507100 & 0.56112200 \\
\hline $\mathrm{C}$ & -0.32845300 & 5. 24203800 & 1. 64428600 \\
\hline $\mathrm{C}$ & -1.56302300 & 4. 53560700 & 2. 23677200 \\
\hline $\mathrm{C}$ & -0.80569000 & 6. 15904300 & 0.49332500 \\
\hline $\mathrm{C}$ & 0.31733500 & 6. 10154000 & 2. 75651100 \\
\hline $\mathrm{H}$ & 0.03580600 & -1.34382800 & -1.82482500 \\
\hline $\mathrm{H}$ & -3.02524600 & 0.47429000 & 0.84127800 \\
\hline $\mathrm{H}$ & -3.07406300 & -1.30336900 & 2. 48436400 \\
\hline $\mathrm{H}$ & -4.56674800 & -1.83600600 & 1. 63191800 \\
\hline $\mathrm{H}$ & -1.95361500 & -3.51021000 & -2.23493500 \\
\hline $\mathrm{H}$ & -0.28673900 & -5.09227700 & -3.18648100 \\
\hline $\mathrm{H}$ & 1. 75582600 & -5.69379400 & -1.87592800 \\
\hline $\mathrm{H}$ & 2. 21860900 & -5.73706200 & 1. 20052800 \\
\hline $\mathrm{H}$ & 3.32483800 & -4.67475900 & 0.32046000 \\
\hline $\mathrm{H}$ & 2.74526100 & -2.76231200 & 1. 62033500 \\
\hline $\mathrm{H}$ & 2. 42187700 & -3.97012200 & 2. 87053400 \\
\hline $\mathrm{H}$ & -1.26379200 & -4.19913000 & 2. 35236500 \\
\hline $\mathrm{H}$ & 0.14709400 & -5.23570200 & 2. 64417800 \\
\hline $\mathrm{H}$ & -1.04299300 & -3.82319900 & 4. 71902400 \\
\hline $\mathrm{H}$ & 0.71930400 & -3.96887600 & 4. 67138300 \\
\hline $\mathrm{H}$ & -0.46893200 & -1.37800300 & 5. 80075400 \\
\hline $\mathrm{H}$ & -0.01318900 & 1. 00773000 & 5. 13939100 \\
\hline $\mathrm{H}$ & 0.66961000 & 1. 52291500 & 2. 86424400 \\
\hline $\mathrm{H}$ & 3. 42108400 & -0.05037600 & 2. 05044000 \\
\hline $\mathrm{H}$ & 6.28993400 & -1.95986300 & -0.51763600 \\
\hline $\mathrm{H}$ & 2. 29340500 & -1.22506000 & -1.91869600 \\
\hline $\mathrm{H}$ & 3. 22529900 & -1.29638600 & -3.97880700 \\
\hline $\mathrm{H}$ & 2. 73727800 & -2.89807300 & -3.37014800 \\
\hline $\mathrm{H}$ & 3.92114100 & -2.76548400 & -4.68838100 \\
\hline $\mathrm{H}$ & 6. 09798200 & -3.79008900 & -1.83825900 \\
\hline $\mathrm{H}$ & 5.53780700 & -4.19033700 & -3.47588100 \\
\hline $\mathrm{H}$ & 4. 42886800 & -4.35461500 & -2.09133300 \\
\hline $\mathrm{H}$ & 5. 59612300 & -0.40965100 & -3.52398200 \\
\hline $\mathrm{H}$ & 6. 20928200 & -1.87788000 & -4.32674900 \\
\hline $\mathrm{H}$ & 6. 79689300 & -1.43738700 & -2.70786800 \\
\hline $\mathrm{H}$ & 5. 29842000 & 0.80092900 & 3. 07176500 \\
\hline $\mathrm{H}$ & 6. 38650300 & -0.22814600 & 4. 02648900 \\
\hline $\mathrm{H}$ & 4. 71653300 & -0.74931300 & 3. 73148000 \\
\hline $\mathrm{H}$ & 5.58444100 & -2.96314300 & 2. 77511600 \\
\hline $\mathrm{H}$ & 7. 23246200 & -2.40956400 & 3. 16965500 \\
\hline $\mathrm{H}$ & 6.85172500 & -2.97231700 & 1. 52616800 \\
\hline $\mathrm{H}$ & 7. 07854300 & 0.81535300 & 1. 20077200 \\
\hline
\end{tabular}




\begin{tabular}{|c|c|c|c|}
\hline H & 7. 76150700 & -0.71454500 & 0.60310100 \\
\hline $\mathrm{H}$ & 8. 09873500 & -0.18595600 & 2. 26410900 \\
\hline $\mathrm{H}$ & -0.51582100 & 2. 48356800 & 1. 28370100 \\
\hline $\mathrm{H}$ & 2. 15709100 & 5.78004000 & 0.68802100 \\
\hline $\mathrm{H}$ & 3. 46882200 & 1. 78720500 & -0.17957800 \\
\hline $\mathrm{H}$ & 4. 53109500 & 2.81526500 & -1.88520600 \\
\hline H & 5.51510600 & 4. 26822800 & -2.16569500 \\
\hline $\mathrm{H}$ & 3. 75434300 & 4. 31488800 & -2.44279000 \\
\hline $\mathrm{H}$ & 5. 41713500 & 6.22018700 & -0.66190900 \\
\hline H & 4. 33275100 & 6.29391600 & 0.74145400 \\
\hline $\mathrm{H}$ & 3. 66584000 & 6.38078400 & -0.90995200 \\
\hline H & 5. 46696200 & 2. 68457300 & 0.54221100 \\
\hline $\mathrm{H}$ & 5. 33871200 & 4. 10218100 & 1. 60949500 \\
\hline H & 6. 44454400 & 4. 13318200 & 0.21096500 \\
\hline H & -1.28641100 & 3. 85361500 & 3. 05790500 \\
\hline $\mathrm{H}$ & -2.12197400 & 3.97445500 & 1. 47312800 \\
\hline $\mathrm{H}$ & -2.24841600 & 5. 29041100 & 2. 65455900 \\
\hline $\mathrm{H}$ & 0.03486300 & 6.67762200 & 0.00499400 \\
\hline $\mathrm{H}$ & -1.49304000 & 6.92864000 & 0.88244500 \\
\hline $\mathrm{H}$ & -1.35228600 & 5.57731500 & -0.26367700 \\
\hline $\mathrm{H}$ & 1. 16384400 & 6.70135600 & 2. 38819100 \\
\hline $\mathrm{H}$ & 0.68661600 & 5. 47192400 & 3. 58262100 \\
\hline $\mathrm{H}$ & -0.42507100 & 6.80252300 & 3. 17143500 \\
\hline $\mathrm{C}$ & -4.34246900 & -0.38925000 & -0.64522900 \\
\hline $\mathrm{H}$ & -4.50239700 & -1.37332900 & -1.10835000 \\
\hline $\mathrm{H}$ & -3.93477500 & 0.24331200 & -1.44410100 \\
\hline $\mathrm{C}$ & -5.63147400 & 0.19262500 & -0.09829000 \\
\hline $\mathrm{C}$ & -6.78039600 & -0.62360300 & 0.19557700 \\
\hline $\mathrm{C}$ & -5.69513600 & 1. 55899800 & 0.13996400 \\
\hline $\mathrm{C}$ & -6.82169300 & -2.03493000 & -0.01910000 \\
\hline $\mathrm{C}$ & -7.96359500 & 0.00133200 & 0.73072700 \\
\hline $\mathrm{C}$ & -6.86056000 & 2. 16380900 & 0.66794900 \\
\hline $\mathrm{H}$ & -4.83103800 & 2. 19283400 & -0.08740200 \\
\hline $\mathrm{C}$ & -7.94489700 & -2.78079600 & 0.27594600 \\
\hline $\mathrm{H}$ & -5.94894100 & -2.54369200 & -0.43042800 \\
\hline $\mathrm{C}$ & -9.10548300 & -0.79813500 & 1. 02193800 \\
\hline $\mathrm{C}$ & -7.97200300 & 1. 40431800 & 0.95841300 \\
\hline $\mathrm{H}$ & -6.86843300 & 3. 24358100 & 0.83723300 \\
\hline $\mathrm{C}$ & -9.10262200 & -2.15855700 & 0.80314800 \\
\hline $\mathrm{H}$ & -7.94215400 & -3.85923700 & 0.09684100 \\
\hline $\mathrm{H}$ & -9.99459000 & -0.30568900 & 1. 42612700 \\
\hline $\mathrm{H}$ & -8.87623500 & 1.86676700 & 1. 36413100 \\
\hline $\mathrm{H}$ & -9.98824700 & -2.75709600 & 1. 03145500 \\
\hline $\mathrm{C}$ & -0.77522500 & 0.70825000 & -4.85187900 \\
\hline 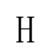 & -0.97417600 & 1. 14226000 & -5.84567900 \\
\hline
\end{tabular}




$\begin{array}{rrrr}\mathrm{H} & -0.41770500 & -0.32376500 & -4.99503000 \\ \mathrm{H} & 0.04129200 & 1.29463100 & -4.39873800 \\ \mathrm{C} & -2.05167600 & 0.70463000 & -3.99109000 \\ \mathrm{O} & -1.97825400 & -0.09032300 & -2.84645700 \\ \mathrm{C} & -2.57751400 & 2.13519000 & -3.72272100 \\ \mathrm{H} & -2.60149600 & 2.67621500 & -4.68555500 \\ \mathrm{H} & -3.62767000 & 2.06645700 & -3.39157700 \\ \mathrm{C} & -1.80599600 & 2.99141100 & -2.69602800 \\ \mathrm{H} & -0.71697800 & 2.86630500 & -2.81833100 \\ \mathrm{H} & -2.03549500 & 4.05019400 & -2.88061900 \\ \mathrm{C} & -2.21391800 & 2.72279400 & -1.24264200 \\ 0 & -3.02599800 & 3.47914400 & -0.70485600 \\ 0 & -1.70335800 & 1.70833700 & -0.61383300 \\ \mathrm{H} & -2.82861900 & 0.22559500 & -4.62663600 \\ \mathrm{H} & 0.58631400 & 0.09744000 & -2.46572700 \\ \mathrm{H} & 0.34463600 & 1.17123400 & -2.08372600\end{array}$

\section{IntLR2}

Ir

$\mathrm{P}$

0

N

C

C

C

C

C

C

C

C

C

C

C

C

C

C

C

C

C

C

C

C

C

C

$\begin{array}{rrr}-0.54005800 & -0.00969500 & -1.38748900 \\ 1.04912400 & 0.14248200 & 0.37621100 \\ -2.88024700 & -2.81830900 & 1.06318100 \\ -2.06981500 & -1.14134400 & -0.17458100 \\ -3.29865700 & -0.58457300 & 0.43883900 \\ -3.60596300 & -1.63940800 & 1.51910500 \\ -1.97834900 & -2.36997900 & 0.17535200 \\ -0.96721300 & -3.31384800 & -0.35811300 \\ -1.17979100 & -3.87468600 & -1.62961500 \\ -0.22169100 & -4.71311900 & -2.20366400 \\ 0.96188200 & -5.00061600 & -1.51521200 \\ 1.17540300 & -4.44135100 & -0.25389700 \\ 2.32533800 & -4.69793300 & 0.69061600 \\ 2.12658700 & -3.64328600 & 1.79627600 \\ 0.60785200 & -3.26148600 & 1.76952900 \\ 0.22487900 & -3.58437700 & 0.32836000 \\ -0.20889500 & -4.23676100 & 2.68127200 \\ -0.12904600 & -3.63965500 & 4.08369600 \\ 0.01529200 & -2.16366500 & 3.80996700 \\ -0.13266500 & -1.16909300 & 4.77568100 \\ 0.12181700 & 0.14893600 & 4.41854500 \\ 0.49508700 & 0.44625500 & 3.10710100 \\ 0.61876800 & -0.52900900 & 2.09521800 \\ 0.37966400 & -1.88966500 & 2.46846400 \\ 2.74710300 & -0.55577300 & 0.10284600 \\ 3.68319500 & -0.49136400 & 1.14961200\end{array}$

S137 


\begin{tabular}{|c|c|c|c|}
\hline $\mathrm{C}$ & 4. 99325700 & -0.95855500 & 0.98293400 \\
\hline $\mathrm{C}$ & 5.33652000 & -1.49260000 & -0.27244400 \\
\hline $\mathrm{C}$ & 4. 43558100 & -1.56736200 & -1.34459600 \\
\hline $\mathrm{C}$ & 3. 13099600 & -1.08802000 & -1.13200000 \\
\hline $\mathrm{C}$ & 4. 89623900 & -2.14553000 & -2.69898400 \\
\hline $\mathrm{C}$ & 3. 75320100 & -2.19295600 & -3.72931900 \\
\hline $\mathrm{C}$ & 5. 42331800 & -3.58521000 & -2.49855600 \\
\hline $\mathrm{C}$ & 6. 02895600 & -1.26078900 & -3.27035200 \\
\hline $\mathrm{C}$ & 6.04087800 & -0.92295500 & 2. 11552800 \\
\hline $\mathrm{C}$ & 5.50295300 & -0.23371400 & 3. 38257700 \\
\hline $\mathrm{C}$ & 6.43947000 & -2.37142700 & 2. 48299800 \\
\hline $\mathrm{C}$ & 7. 29451900 & -0.15035000 & 1. 64416900 \\
\hline $\mathrm{C}$ & 1. 42976400 & 1. 95645200 & 0.59271100 \\
\hline $\mathrm{C}$ & 0.44449800 & 2. 84725700 & 1. 06708400 \\
\hline $\mathrm{C}$ & 0.68366400 & 4. 22323200 & 1. 14041200 \\
\hline $\mathrm{C}$ & 1. 92005600 & 4. 70248700 & 0.66352500 \\
\hline $\mathrm{C}$ & 2.90758400 & 3. 85810900 & 0.14804500 \\
\hline $\mathrm{C}$ & 2. 64646700 & 2. 47448100 & 0.13592000 \\
\hline $\mathrm{C}$ & 4. 25343700 & 4. 37942500 & -0.39823800 \\
\hline $\mathrm{C}$ & 4. 39752800 & 3. 96832900 & -1.88217400 \\
\hline $\mathrm{C}$ & 4. 36486200 & 5. 91257500 & -0.31310500 \\
\hline $\mathrm{C}$ & 5. 41466500 & 3. 76709300 & 0.41869800 \\
\hline $\mathrm{C}$ & -0.35293400 & 5.21460400 & 1. 71108200 \\
\hline $\mathrm{C}$ & -1.57694000 & 4. 49677000 & 2. 31121300 \\
\hline $\mathrm{C}$ & -0.85108400 & 6.14549200 & 0.58017000 \\
\hline $\mathrm{C}$ & 0.30776600 & 6. 05990700 & 2. 82505900 \\
\hline $\mathrm{H}$ & -0.07573900 & -1.54728400 & -1.81581700 \\
\hline $\mathrm{H}$ & -3.06654500 & 0.39789600 & 0.86749500 \\
\hline $\mathrm{H}$ & -3.20145200 & -1.36780000 & 2. 50692300 \\
\hline $\mathrm{H}$ & -4.66619200 & -1.90330000 & 1. 61215700 \\
\hline $\mathrm{H}$ & -2.09212300 & -3.62650700 & -2.17595100 \\
\hline $\mathrm{H}$ & -0.40048800 & -5.14021400 & -3.19326500 \\
\hline $\mathrm{H}$ & 1. 70817300 & -5.66717500 & -1.95647800 \\
\hline $\mathrm{H}$ & 2. 25864900 & -5.72731500 & 1. 08668400 \\
\hline $\mathrm{H}$ & 3.30854600 & -4.61346900 & 0.20115300 \\
\hline $\mathrm{H}$ & 2. 72708300 & -2.75597200 & 1. 56758700 \\
\hline $\mathrm{H}$ & 2. 43623100 & -3.99642100 & 2. 79147000 \\
\hline $\mathrm{H}$ & -1.25536500 & -4.24342900 & 2. 34424600 \\
\hline $\mathrm{H}$ & 0.16994600 & -5.26748800 & 2. 61042400 \\
\hline $\mathrm{H}$ & -1.00893500 & -3.87226500 & 4. 70462200 \\
\hline $\mathrm{H}$ & 0.75359600 & -4.00748000 & 4. 63799600 \\
\hline $\mathrm{H}$ & -0.42455200 & -1.43264600 & 5. 79598400 \\
\hline $\mathrm{H}$ & 0.03747200 & 0.95628300 & 5. 15015800 \\
\hline $\mathrm{H}$ & 0.70343600 & 1. 48614200 & 2. 87354800 \\
\hline $\mathrm{H}$ & 3. 37242200 & -0.06712600 & 2. 10338700 \\
\hline
\end{tabular}




\begin{tabular}{|c|c|c|c|}
\hline H & 6. 35329900 & -1.86159300 & -0.42003700 \\
\hline $\mathrm{H}$ & 2. 40597600 & -1.11892300 & -1.94021000 \\
\hline $\mathrm{H}$ & 3. 35859500 & -1.19045500 & -3.95598500 \\
\hline $\mathrm{H}$ & 2. 91627300 & -2.82063800 & -3.38524200 \\
\hline H & 4. 12156600 & -2.62113000 & -4.67463700 \\
\hline $\mathrm{H}$ & 6.27703900 & -3.62231300 & -1.80467200 \\
\hline $\mathrm{H}$ & 5. 75984100 & -4.00745700 & -3.45943600 \\
\hline $\mathrm{H}$ & 4. 63502100 & -4.24259000 & -2.09798000 \\
\hline $\mathrm{H}$ & 5. 68187500 & -0.22720600 & -3.42704600 \\
\hline H & 6.37252400 & -1.65458800 & -4.24098000 \\
\hline $\mathrm{H}$ & 6.90019200 & -1.22372300 & -2.59848400 \\
\hline H & 5. 20832500 & 0.80927500 & 3. 18858300 \\
\hline H & 6.28492800 & -0.21882800 & 4. 15783500 \\
\hline $\mathrm{H}$ & 4. 63348900 & -0.76194700 & 3. 80343300 \\
\hline H & 5. 56761000 & -2.94230500 & 2. 84046100 \\
\hline $\mathrm{H}$ & 7. 19646800 & -2.37026400 & 3. 28441300 \\
\hline $\mathrm{H}$ & 6.86496800 & -2.91128800 & 1. 62307200 \\
\hline $\mathrm{H}$ & 7. 04181200 & 0.88663500 & 1. 37220000 \\
\hline $\mathrm{H}$ & 7. 76931800 & -0.62081000 & 0.76966400 \\
\hline $\mathrm{H}$ & 8.04698700 & -0.11610100 & 2. 44879300 \\
\hline $\mathrm{H}$ & -0.52577300 & 2. 45862700 & 1. 36018600 \\
\hline $\mathrm{H}$ & 2. 10372800 & 5. 77538500 & 0.69498900 \\
\hline $\mathrm{H}$ & 3. 40599600 & 1. 79837600 & -0.25308900 \\
\hline $\mathrm{H}$ & 4. 37138600 & 2.87545400 & -2.01030800 \\
\hline $\mathrm{H}$ & 5. 35579800 & 4. 32952600 & -2.29107200 \\
\hline $\mathrm{H}$ & 3.58443800 & 4. 39573800 & -2.49008600 \\
\hline $\mathrm{H}$ & 5. 33576000 & 6.23841100 & -0.71866700 \\
\hline $\mathrm{H}$ & 4. 30368600 & 6.27313700 & 0.72563600 \\
\hline $\mathrm{H}$ & 3. 57799400 & 6.41485300 & -0.89699300 \\
\hline $\mathrm{H}$ & 5. 42069300 & 2. 66786000 & 0.36161400 \\
\hline $\mathrm{H}$ & 5. 34009600 & 4. 04837400 & 1. 48144100 \\
\hline $\mathrm{H}$ & 6. 38559900 & 4. 12637500 & 0.03899100 \\
\hline $\mathrm{H}$ & -1.28952300 & 3.81178600 & 3. 12600400 \\
\hline $\mathrm{H}$ & -2.13347200 & 3. 93370700 & 1. 54757200 \\
\hline $\mathrm{H}$ & -2.26525100 & 5. 24352200 & 2. 73855900 \\
\hline $\mathrm{H}$ & -0.02070300 & 6.67906000 & 0.09084200 \\
\hline $\mathrm{H}$ & -1.54029200 & 6.90346200 & 0.98863300 \\
\hline $\mathrm{H}$ & -1.39859400 & 5.56923400 & -0.18057200 \\
\hline $\mathrm{H}$ & 1. 14947500 & 6.66343800 & 2. 45189200 \\
\hline $\mathrm{H}$ & 0.68877200 & 5. 41961600 & 3. 63751000 \\
\hline $\mathrm{H}$ & -0.42814900 & 6.75607400 & 3.25953000 \\
\hline $\mathrm{C}$ & -4.39083800 & -0.41640900 & -0.64030400 \\
\hline $\mathrm{H}$ & -4.58985100 & -1.39773900 & -1.09459800 \\
\hline $\mathrm{H}$ & -3.94789000 & 0.18930700 & -1.44282600 \\
\hline 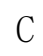 & -5.65751500 & 0.22992200 & -0.11427500 \\
\hline
\end{tabular}




$\begin{array}{lrrr}\mathrm{C} & -6.84710400 & -0.52627500 & 0.17692800 \\ \mathrm{C} & -5.65814100 & 1.60036800 & 0.10801700 \\ \mathrm{C} & -6.95568100 & -1.93565400 & -0.02592300 \\ \mathrm{C} & -8.00299000 & 0.16069700 & 0.69560400 \\ \mathrm{C} & -6.79686100 & 2.26695300 & 0.61916200 \\ \mathrm{H} & -4.76188000 & 2.18717000 & -0.11943200 \\ \mathrm{C} & -8.11608200 & -2.62326400 & 0.26632900 \\ \mathrm{H} & -6.10619100 & -2.48933400 & -0.42761300 \\ \mathrm{C} & -9.18453500 & -0.57962500 & 0.98503600 \\ \mathrm{C} & -7.94569300 & 1.56484100 & 0.90868400 \\ \mathrm{H} & -6.75446100 & 3.34772700 & 0.77662200 \\ \mathrm{C} & -9.24601800 & -1.94062400 & 0.77893000 \\ \mathrm{H} & -8.16481600 & -3.70205300 & 0.09615200 \\ \mathrm{H} & -10.05182400 & -0.04073000 & 1.37717000 \\ \mathrm{H} & -8.82966800 & 2.07460500 & 1.30212600 \\ \mathrm{H} & -10.16139000 & -2.49327200 & 1.00559200 \\ \mathrm{C} & -0.69484600 & 0.59969300 & -4.86635700 \\ \mathrm{H} & -0.83331700 & 1.07868600 & -5.84999200 \\ \mathrm{H} & -0.43338700 & -0.45726200 & -5.03718100 \\ \mathrm{H} & 0.16111000 & 1.08433400 & -4.37036400 \\ \mathrm{C} & -1.97850300 & 0.68863100 & -4.02323200 \\ \mathrm{O} & -1.96306100 & -0.09852500 & -2.87013700 \\ \mathrm{C} & -2.40909500 & 2.15050500 & -3.76021500 \\ \mathrm{H} & -2.36001900 & 2.70573000 & -4.71374800 \\ \mathrm{H} & -3.47100800 & 2.15677900 & -3.46065000 \\ \mathrm{C} & -1.60683000 & 2.92836000 & -2.69595900 \\ \mathrm{H} & -0.52655800 & 2.74758600 & -2.80566800 \\ \mathrm{H} & -1.78540400 & 4.00301500 & -2.84197000 \\ \mathrm{C} & -2.05130500 & 2.63111300 & -1.26474000 \\ \text { O } & -2.86224500 & 3.37296200 & -0.71456000 \\ \text { O } & -1.56180900 & 1.59617400 & -0.62987900 \\ \mathrm{H} & -2.78207000 & 0.25856800 & -4.66072900 \\ \mathrm{H} & 0.41970900 & -0.93813600 & -2.34195700 \\ \mathrm{H} & 0.44949300 & 0.92596100 & -2.18299800\end{array}$

\section{TSLR2}

Ir

$\mathrm{P}$

0

N

C

C

C

C
0. 47611300

$-1.02523000$

2. 89676400

2. 05918900

3. 27432600

3. 59956200

1. 98772000

0. 98385500
$-0.10302600$

0. 18269400

$-2.78598600$

$-1.16469000$

$-0.56589900$

$-1.58080300$

$-2.38220000$

$-3.35098900$
1. 35362500

$-0.38990300$

$-1.13279700$

0. 16190800

$-0.43747900$

$-1.55094400$

-0. 22959800

0. 26880700 


\begin{tabular}{|c|c|c|c|}
\hline $\mathrm{C}$ & 1. 20030800 & -3.96289400 & 1. 51388700 \\
\hline $\mathrm{C}$ & 0.23789700 & -4.81286500 & 2. 06287500 \\
\hline $\mathrm{C}$ & -0.95370700 & -5.06097700 & 1. 37357100 \\
\hline $\mathrm{C}$ & -1.16880300 & -4.45358700 & 0.13509100 \\
\hline $\mathrm{C}$ & -2.32684400 & -4.66526200 & -0.81060600 \\
\hline $\mathrm{C}$ & -2.12145700 & -3.57698100 & -1.88193000 \\
\hline $\mathrm{C}$ & -0.59924600 & -3.21165200 & -1.84978300 \\
\hline $\mathrm{C}$ & -0.21381200 & -3.58436900 & -0.42024400 \\
\hline $\mathrm{C}$ & 0.20517000 & -4.17164400 & -2.78894200 \\
\hline $\mathrm{C}$ & 0.13128300 & -3.53797300 & -4.17479000 \\
\hline $\mathrm{C}$ & 0.01390800 & -2.06786300 & -3.86093200 \\
\hline $\mathrm{C}$ & 0.19354900 & -1.05109500 & -4.79777100 \\
\hline $\mathrm{C}$ & -0.02805200 & 0.26318000 & -4.40657900 \\
\hline $\mathrm{C}$ & -0.41062000 & 0.53254000 & -3.09201200 \\
\hline $\mathrm{C}$ & -0.57508700 & -0.46814300 & -2.11068400 \\
\hline $\mathrm{C}$ & -0.35535700 & -1.82372900 & -2.51451000 \\
\hline $\mathrm{C}$ & -2.72854900 & -0.49481400 & -0.12290800 \\
\hline $\mathrm{C}$ & -3.65268000 & -0.46045400 & -1.18140700 \\
\hline C & -4.96967300 & -0.90755400 & -1.00867500 \\
\hline $\mathrm{C}$ & -5.32971900 & -1.38792200 & 0.26322000 \\
\hline $\mathrm{C}$ & -4.43964200 & -1.42848400 & 1. 34658000 \\
\hline $\mathrm{C}$ & -3.12827500 & -0.97164800 & 1. 12962000 \\
\hline $\mathrm{C}$ & -4.91725800 & -1.95344400 & 2. 71635600 \\
\hline $\mathrm{C}$ & -3.79365900 & -1.93538500 & 3. 76850100 \\
\hline $\mathrm{C}$ & -5.41504100 & -3.40981900 & 2. 56728900 \\
\hline $\mathrm{C}$ & -6.07502800 & -1.06513200 & 3. 22859400 \\
\hline $\mathrm{C}$ & -6.00802200 & -0.90436800 & -2.15074700 \\
\hline $\mathrm{C}$ & -5.44939400 & -0.27915500 & -3.44176500 \\
\hline $\mathrm{C}$ & -6.42792600 & -2.36056100 & -2.45980200 \\
\hline $\mathrm{C}$ & -7.25244200 & -0.09270800 & -1.72234000 \\
\hline $\mathrm{C}$ & -1.36227300 & 2. 00583800 & -0.59117800 \\
\hline $\mathrm{C}$ & -0.31648000 & 2. 88226000 & -0.94953200 \\
\hline $\mathrm{C}$ & -0.51870900 & 4. 26429000 & -1.01430200 \\
\hline $\mathrm{C}$ & -1.78998200 & 4. 76121700 & -0.66380300 \\
\hline $\mathrm{C}$ & -2.84412700 & 3. 92957300 & -0.27611900 \\
\hline $\mathrm{C}$ & -2.61068400 & 2.54078500 & -0.25593500 \\
\hline $\mathrm{C}$ & -4.23542600 & 4. 47160200 & 0.11495300 \\
\hline $\mathrm{C}$ & -4.58029800 & 4. 02253300 & 1. 55404500 \\
\hline $\mathrm{C}$ & -4.29877400 & 6.00894000 & 0.06593200 \\
\hline $\mathrm{C}$ & -5.29484100 & 3. 91514200 & -0.86431700 \\
\hline $\mathrm{C}$ & 0.59678500 & 5.24624100 & -1.43213400 \\
\hline $\mathrm{C}$ & 1. 86098200 & 4. 51595900 & -1.92200500 \\
\hline $\mathrm{C}$ & 0.98917900 & 6.11766200 & -0.21538700 \\
\hline $\mathrm{C}$ & 0.08515400 & 6.15302600 & -2.57547000 \\
\hline $\mathrm{H}$ & 0.43137000 & -1.29206300 & 2. 57191400 \\
\hline
\end{tabular}




\begin{tabular}{|c|c|c|c|}
\hline H & 3. 02334700 & 0.42424100 & -0.83712000 \\
\hline $\mathrm{H}$ & 3. 18644500 & -1.28236600 & -2.52742000 \\
\hline $\mathrm{H}$ & 4. 66348300 & -1.82233900 & -1.65808400 \\
\hline $\mathrm{H}$ & 2. 11636000 & -3.74170600 & 2. 06545000 \\
\hline H & 0.41867400 & -5.27899700 & 3. 03445600 \\
\hline $\mathrm{H}$ & -1.70475400 & -5.73504700 & 1. 79506600 \\
\hline $\mathrm{H}$ & -2.27602700 & -5.68171500 & -1.24104500 \\
\hline $\mathrm{H}$ & -3.30619900 & -4.58468700 & -0.31254600 \\
\hline $\mathrm{H}$ & -2.71092100 & -2.69187100 & -1.61970600 \\
\hline H & -2.44010900 & -3.89314300 & -2.88683700 \\
\hline $\mathrm{H}$ & 1. 25208500 & -4.19795500 & -2.45369400 \\
\hline H & -0.18437600 & -5.19975700 & -2.74370500 \\
\hline H & 1. 00445200 & -3.76961600 & -4.80545300 \\
\hline $\mathrm{H}$ & -0.76025300 & -3.87510000 & -4.73449200 \\
\hline H & 0.48784300 & -1.29443900 & -5.82242800 \\
\hline $\mathrm{H}$ & 0.08778000 & 1. 08813600 & -5.11383500 \\
\hline H & -0.59325300 & 1. 57067200 & -2.82910900 \\
\hline $\mathrm{H}$ & -3.32871300 & -0.07808700 & -2.14862900 \\
\hline $\mathrm{H}$ & -6.35130700 & -1.74167600 & 0.41536700 \\
\hline H & -2.40362000 & -0.97379300 & 1. 94003100 \\
\hline $\mathrm{H}$ & -3.41382300 & -0.91822100 & 3. 95008400 \\
\hline H & -2.94280700 & -2.56817400 & 3. 47204100 \\
\hline $\mathrm{H}$ & -4.17556300 & -2.32041200 & 4. 72700200 \\
\hline $\mathrm{H}$ & -6.25576000 & -3.49019000 & 1. 86109800 \\
\hline $\mathrm{H}$ & -5.76067900 & -3.79897700 & 3. 53900400 \\
\hline $\mathrm{H}$ & -4.60790900 & -4.06753400 & 2. 20670100 \\
\hline $\mathrm{H}$ & -5.74779200 & -0.01978200 & 3. 34521300 \\
\hline $\mathrm{H}$ & -6.42886300 & -1.42206900 & 4. 20977100 \\
\hline $\mathrm{H}$ & -6.93580700 & -1.07245800 & 2. 54225800 \\
\hline H & -5.14205400 & 0.76735300 & -3.29143800 \\
\hline $\mathrm{H}$ & -6.22385300 & -0.28826100 & -4.22464300 \\
\hline $\mathrm{H}$ & -4.58331300 & -0.83736600 & -3.82940900 \\
\hline $\mathrm{H}$ & -5.56319500 & -2.96043700 & -2.78572000 \\
\hline $\mathrm{H}$ & -7.17907900 & -2.38142900 & -3.26647800 \\
\hline $\mathrm{H}$ & -6.86844900 & -2.85664800 & -1.58132900 \\
\hline $\mathrm{H}$ & -6.98504000 & 0.95115500 & -1.49436700 \\
\hline $\mathrm{H}$ & -7.73831600 & -0.51702600 & -0.83065600 \\
\hline $\mathrm{H}$ & -7.99995400 & -0.08226600 & -2.53228100 \\
\hline $\mathrm{H}$ & 0.67085700 & 2. 47775600 & -1.15203800 \\
\hline $\mathrm{H}$ & -1.94798900 & 5. 83822100 & -0.69350700 \\
\hline $\mathrm{H}$ & -3.41879800 & 1.87393600 & 0.03932800 \\
\hline $\mathrm{H}$ & -4.60080300 & 2. 92636100 & 1. 65048200 \\
\hline $\mathrm{H}$ & -5.57332400 & 4. 40138800 & 1. 84768300 \\
\hline $\mathrm{H}$ & -3.84196900 & 4. 40786200 & 2. 27504700 \\
\hline 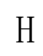 & -5.30517500 & 6. 34997800 & 0.35606800 \\
\hline
\end{tabular}




\begin{tabular}{|c|c|c|c|}
\hline H & -4.09859400 & 6. 39789200 & -0.94465700 \\
\hline $\mathrm{H}$ & -3.57979200 & 6.47199600 & 0.75954400 \\
\hline $\mathrm{H}$ & -5.32765800 & 2.81508800 & -0.84768700 \\
\hline $\mathrm{H}$ & -5.07882300 & 4. 22933300 & -1.89806700 \\
\hline H & -6.29964500 & 4. 28408600 & -0.59928500 \\
\hline $\mathrm{H}$ & 1. 64738100 & 3. 86512400 & -2.78593200 \\
\hline $\mathrm{H}$ & 2. 31790200 & 3. 91562000 & -1.12142500 \\
\hline $\mathrm{H}$ & 2. 60890100 & 5.25753700 & -2.24578700 \\
\hline $\mathrm{H}$ & 0.12343800 & 6.65934600 & 0.19771800 \\
\hline H & 1. 74247800 & 6.86648900 & -0.51217000 \\
\hline $\mathrm{H}$ & 1. 42935400 & 5. 49599900 & 0.57853600 \\
\hline H & -0.77774800 & 6.76634800 & -2.27286000 \\
\hline H & -0.21736300 & 5.55705000 & -3.45208900 \\
\hline $\mathrm{H}$ & 0.88211100 & 6.84402800 & -2.89506200 \\
\hline $\mathrm{C}$ & 4. 36511800 & -0.39815500 & 0.64448000 \\
\hline $\mathrm{H}$ & 4. 58137800 & -1.38104600 & 1. 08787300 \\
\hline $\mathrm{H}$ & 3.91894200 & 0.20240700 & 1. 44778700 \\
\hline $\mathrm{C}$ & 5. 62034900 & 0.28468300 & 0.13635100 \\
\hline $\mathrm{C}$ & 6.80367500 & -0.44730000 & -0.23031700 \\
\hline $\mathrm{C}$ & 5. 61280300 & 1. 66684100 & 0.00687900 \\
\hline $\mathrm{C}$ & 6.92346800 & -1.86502500 & -0.10930800 \\
\hline $\mathrm{C}$ & 7. 94243000 & 0.27512800 & -0.73787800 \\
\hline $\mathrm{C}$ & 6. 73551000 & 2. 36854900 & -0.49284900 \\
\hline $\mathrm{H}$ & 4. 72522500 & 2. 23380700 & 0.30833300 \\
\hline $\mathrm{C}$ & 8.07842500 & -2.52829000 & -0.47134500 \\
\hline $\mathrm{H}$ & 6. 08801900 & -2.44500900 & 0.28492300 \\
\hline $\mathrm{C}$ & 9. 11783500 & -0.44105300 & -1.10298200 \\
\hline $\mathrm{C}$ & 7. 87540100 & 1. 68962300 & -0.86205000 \\
\hline $\mathrm{H}$ & 6.68817700 & 3. 45722800 & -0.57729400 \\
\hline $\mathrm{C}$ & 9. 19015200 & -1.81129900 & -0.97687200 \\
\hline $\mathrm{H}$ & 8. 13701200 & -3.61448100 & -0.36271600 \\
\hline $\mathrm{H}$ & 9.97186000 & 0.12395700 & -1.48731700 \\
\hline $\mathrm{H}$ & 8. 74632200 & 2. 22628300 & -1.24874000 \\
\hline $\mathrm{H}$ & 10. 10072900 & -2.34512000 & -1.26062500 \\
\hline $\mathrm{C}$ & 0.34543700 & 0.20319600 & 4. 98426100 \\
\hline $\mathrm{H}$ & 0.45642800 & 0.69826900 & 5.96263700 \\
\hline $\mathrm{H}$ & -0.01943400 & -0.82117100 & 5. 16425200 \\
\hline $\mathrm{H}$ & -0.42535700 & 0.74170300 & 4. 41246900 \\
\hline $\mathrm{C}$ & 1. 68649100 & 0.15970800 & 4. 24270700 \\
\hline 0 & 1. 65408300 & -0.62042900 & 3. 06469100 \\
\hline $\mathrm{C}$ & 2. 30249400 & 1.55665700 & 4. 01900900 \\
\hline $\mathrm{H}$ & 2. 38113600 & 2. 03088200 & 5. 01289500 \\
\hline $\mathrm{H}$ & 3. 34214800 & 1. 42780500 & 3. 67301900 \\
\hline $\mathrm{C}$ & 1.57548200 & 2.54244400 & 3. 07130100 \\
\hline $\mathrm{H}$ & 0.48551800 & 2. 39845500 & 3. 11437000 \\
\hline
\end{tabular}




$\begin{array}{rrrr}\mathrm{H} & 1.79256400 & 3.56529700 & 3.40878300 \\ \mathrm{C} & 2.04895800 & 2.49276900 & 1.61484100 \\ \mathrm{O} & 2.87420100 & 3.31579200 & 1.22072200 \\ \mathrm{O} & 1.58160000 & 1.57328200 & 0.81578100 \\ \mathrm{H} & 2.39517200 & -0.36903300 & 4.91382400 \\ \mathrm{H} & -0.28260700 & -1.51148600 & 1.88437500 \\ \mathrm{H} & -0.59868100 & 0.70471700 & 2.18566200\end{array}$

\section{TSMR2}

$\begin{array}{rrrr}\text { Ir } & -0.51339400 & -0.32711000 & -1.52377400 \\ \text { P } & 0.85092600 & 0.08057300 & 0.33647400 \\ \text { O } & -2.90713600 & -2.78985800 & 0.94598100 \\ \text { N } & -1.95250000 & -1.22139800 & -0.32099200 \\ \text { C } & -3.19474300 & -0.58117000 & 0.20541600 \\ \text { C } & -3.53909000 & -1.53996100 & 1.35234100 \\ \text { C } & -1.95120400 & -2.45024600 & 0.07434500 \\ \text { C } & -0.99422400 & -3.47648700 & -0.39273500 \\ \text { C } & -1.21642400 & -4.10360100 & -1.62859600 \\ \text { C } & -0.27151800 & -4.98724400 & -2.15265700 \\ \text { C } & 0.91453800 & -5.24212700 & -1.45576900 \\ \text { C } & 1.13300000 & -4.62212600 & -0.22459500 \\ \text { C } & 2.29497700 & -4.80861000 & 0.72286900 \\ \text { C } & 2.08599600 & -3.69408300 & 1.76803000 \\ \text { C } & 0.55613300 & -3.36980400 & 1.74534700 \\ \text { C } & 0.18336700 & -3.73941100 & 0.31515100 \\ \text { C } & -0.21953800 & -4.37282500 & 2.66523400 \\ \text { C } & -0.16681300 & -3.76154300 & 4.06066800 \\ \text { C } & -0.12744200 & -2.28400700 & 3.76535600 \\ \text { C } & -0.39352200 & -1.29231300 & 4.70710600 \\ \text { C } & -0.27093000 & 0.03737300 & 4.32503300 \\ \text { C } & 0.10921700 & 0.34276900 & 3.01831500 \\ \text { C } & 0.37178100 & -0.63391100 & 2.03246000 \\ \text { C } & 0.24452400 & -2.00431900 & 2.42503600 \\ \text { C } & 2.59545100 & -0.48781400 & 0.11432600 \\ \text { C } & 3.48558000 & -0.47376300 & 1.20274200 \\ \text { C } & 4.82335000 & -0.85841900 & 1.04257200 \\ \text { C } & 5.23397700 & -1.26059400 & -0.24199000 \\ \text { C } & 4.37278100 & -1.29017700 & -1.34867100 \\ \text { C } & 3.03992300 & -0.88797400 & -1.15085700 \\ \text { C } & 4.89547600 & -1.71752400 & -2.73649300 \\ \text { C } & 3.74407200 & -1.98088600 & -3.72638800 \\ \text { C } & 5.73163100 & -3.01220900 & -2.62354600 \\ \text { C } & 5.78578700 & -0.58464300 & -3.29957800 \\ \text { C } & 5.8557300 & -0.86888200 & 2.20800500\end{array}$




\begin{tabular}{|c|c|c|c|}
\hline $\mathrm{C}$ & 5. 20743300 & -0.38835100 & 3. 52898900 \\
\hline $\mathrm{C}$ & 6.36023000 & -2.30829200 & 2. 41947100 \\
\hline $\mathrm{C}$ & 7. 02210900 & 0.06356700 & 1. 87131700 \\
\hline $\mathrm{C}$ & 1. 03038800 & 1. 90871400 & 0.64173100 \\
\hline $\mathrm{C}$ & -0.12538200 & 2. 68056700 & 0.88585300 \\
\hline $\mathrm{C}$ & -0.03765400 & 4. 05135300 & 1. 15093200 \\
\hline $\mathrm{C}$ & 1. 23696900 & 4. 65110300 & 1. 07992300 \\
\hline $\mathrm{C}$ & 2. 40074700 & 3. 92933600 & 0.79980700 \\
\hline $\mathrm{C}$ & 2. 27693900 & 2.54016500 & 0.60030200 \\
\hline $\mathrm{C}$ & 3. 79526100 & 4. 58675800 & 0.73058800 \\
\hline $\mathrm{C}$ & 4. 42801100 & 4. 31954200 & -0.65455600 \\
\hline $\mathrm{C}$ & 3. 73407500 & 6.11030900 & 0.94315800 \\
\hline $\mathrm{C}$ & 4. 69795100 & 3. 98271100 & 1. 83132700 \\
\hline $\mathrm{C}$ & -1.26368300 & 4. 91020900 & 1. 53046800 \\
\hline $\mathrm{C}$ & -2.51988300 & 4. 05094900 & 1. 76643900 \\
\hline $\mathrm{C}$ & -1.56793300 & 5. 91174700 & 0.39164800 \\
\hline $\mathrm{C}$ & -0.95957500 & 5.68498900 & 2. 83464800 \\
\hline $\mathrm{H}$ & 0.29437400 & -1.66335400 & -1.63538800 \\
\hline $\mathrm{H}$ & -2.96308600 & 0.43140200 & 0.55289300 \\
\hline $\mathrm{H}$ & -3.08054100 & -1.23952700 & 2. 30788500 \\
\hline $\mathrm{H}$ & -4.60977600 & -1.72487400 & 1. 49166400 \\
\hline $\mathrm{H}$ & -2.12303900 & -3.87354300 & -2.19249500 \\
\hline $\mathrm{H}$ & -0.45548100 & -5.46630100 & -3.11716700 \\
\hline $\mathrm{H}$ & 1. 65960600 & -5.92753900 & -1.86917700 \\
\hline $\mathrm{H}$ & 2. 25446000 & -5.81404400 & 1. 17941900 \\
\hline $\mathrm{H}$ & 3.27115500 & -4.73288800 & 0.21797300 \\
\hline $\mathrm{H}$ & 2. 64782700 & -2.80221700 & 1. 46806800 \\
\hline $\mathrm{H}$ & 2. 42956500 & -3.97394200 & 2. 77555000 \\
\hline $\mathrm{H}$ & -1.26539200 & -4.42091400 & 2. 32636900 \\
\hline $\mathrm{H}$ & 0.19764600 & -5.38869500 & 2. 60127700 \\
\hline $\mathrm{H}$ & -1.02196000 & -4.04572800 & 4. 69426500 \\
\hline $\mathrm{H}$ & 0.74561400 & -4.06246200 & 4. 60704200 \\
\hline $\mathrm{H}$ & -0.68392400 & -1.56503000 & 5. 72537500 \\
\hline $\mathrm{H}$ & -0.46223300 & 0.84739200 & 5. 03314200 \\
\hline $\mathrm{H}$ & 0.21160400 & 1. 39409400 & 2. 76711500 \\
\hline $\mathrm{H}$ & 3. 11764500 & -0.16371300 & 2. 18019200 \\
\hline $\mathrm{H}$ & 6.27315600 & -1.56326200 & -0.38134500 \\
\hline $\mathrm{H}$ & 2. 33801800 & -0.86174100 & -1.98251500 \\
\hline $\mathrm{H}$ & 3. 12344400 & -1.09043200 & -3.90143500 \\
\hline $\mathrm{H}$ & 3. 07695400 & -2.78015100 & -3.36644800 \\
\hline $\mathrm{H}$ & 4. 15691800 & -2.29834100 & -4.69714900 \\
\hline $\mathrm{H}$ & 6.63106600 & -2.88109100 & -2.00322400 \\
\hline $\mathrm{H}$ & 6.07008300 & -3.33244300 & -3.62221700 \\
\hline $\mathrm{H}$ & 5. 13719300 & -3.83223800 & -2.18934500 \\
\hline $\mathrm{H}$ & 5. 20943300 & 0.34623200 & -3.41925000 \\
\hline
\end{tabular}




\begin{tabular}{|c|c|c|c|}
\hline H & 6. 18920200 & -0.86330100 & -4.28729000 \\
\hline $\mathrm{H}$ & 6.63751600 & -0.37013000 & -2.63462000 \\
\hline H & 4. 83227000 & 0.64420200 & 3. 45564400 \\
\hline $\mathrm{H}$ & 5. 96391100 & -0.40796500 & 4. 32922800 \\
\hline $\mathrm{H}$ & 4. 37322400 & -1.03219700 & 3. 84840300 \\
\hline $\mathrm{H}$ & 5.53643800 & -2.99693600 & 2. 66645100 \\
\hline $\mathrm{H}$ & 7. 08608300 & -2.33536000 & 3. 24883000 \\
\hline H & 6.86578000 & -2.69849700 & 1. 52294900 \\
\hline $\mathrm{H}$ & 6.68094400 & 1. 10019200 & 1. 72240100 \\
\hline H & 7. 54764900 & -0.25076300 & 0.95681300 \\
\hline H & 7. 75628000 & 0.06270000 & 2. 69358300 \\
\hline $\mathrm{H}$ & -1.09934100 & 2. 19692100 & 0.87099600 \\
\hline H & 1. 30998700 & 5. 72303600 & 1. 25654100 \\
\hline $\mathrm{H}$ & 3. 16921200 & 1. 94920100 & 0.40213800 \\
\hline H & 4. 54565900 & 3.24401400 & -0.85502500 \\
\hline $\mathrm{H}$ & 5. 42765700 & 4. 78063200 & -0.71453500 \\
\hline $\mathrm{H}$ & 3. 80876200 & 4. 74340100 & -1.46069200 \\
\hline $\mathrm{H}$ & 4. 74738200 & 6.53656000 & 0.87515800 \\
\hline $\mathrm{H}$ & 3. 33310100 & 6.37338800 & 1.93445000 \\
\hline H & 3. 11439100 & 6.60710800 & 0.18043700 \\
\hline $\mathrm{H}$ & 4. 82089900 & 2. 89597000 & 1. 70728000 \\
\hline $\mathrm{H}$ & 4. 27350700 & 4. 16091400 & 2. 83235300 \\
\hline $\mathrm{H}$ & 5. 70173600 & 4. 43806800 & 1. 80134700 \\
\hline $\mathrm{H}$ & -2.36044000 & 3. 30814700 & 2. 56579900 \\
\hline $\mathrm{H}$ & -2.83449700 & 3.53729400 & 0.84651900 \\
\hline $\mathrm{H}$ & -3.35312700 & 4. 69921200 & 2. 08161700 \\
\hline $\mathrm{H}$ & -0.69172300 & 6.53481100 & 0.14995800 \\
\hline $\mathrm{H}$ & -2.38546500 & 6.58817500 & 0.69257900 \\
\hline $\mathrm{H}$ & -1.89112400 & 5. 37788900 & -0.51447600 \\
\hline $\mathrm{H}$ & -0.12033200 & 6.38861900 & 2. 72365800 \\
\hline $\mathrm{H}$ & -0.71457600 & 4. 99664500 & 3. 66021400 \\
\hline $\mathrm{H}$ & -1.84136200 & 6.27332600 & 3. 13582600 \\
\hline $\mathrm{C}$ & -4.25873000 & -0.48772100 & -0.91284500 \\
\hline $\mathrm{H}$ & -4.44052200 & -1.49420000 & -1.31957400 \\
\hline $\mathrm{H}$ & -3.80363700 & 0.11225000 & -1.71079600 \\
\hline $\mathrm{C}$ & -5.54555100 & 0.17763600 & -0.46331500 \\
\hline $\mathrm{C}$ & -6.70609800 & -0.57435500 & -0.06535700 \\
\hline $\mathrm{C}$ & -5.58930700 & 1. 56491400 & -0.42789000 \\
\hline $\mathrm{C}$ & -6.77188900 & -2.00052900 & -0.09420200 \\
\hline $\mathrm{C}$ & -7.87867400 & 0.13618000 & 0.37786200 \\
\hline $\mathrm{C}$ & -6.74597300 & 2. 25320700 & 0.01022200 \\
\hline $\mathrm{H}$ & -4.71791400 & 2. 14457400 & -0.75683700 \\
\hline $\mathrm{C}$ & -7.90770200 & -2.68186000 & 0.29451300 \\
\hline $\mathrm{H}$ & -5.90860800 & -2.57425800 & -0.43443000 \\
\hline $\mathrm{C}$ & -9.03275300 & -0.59843000 & 0.77288800 \\
\hline
\end{tabular}




$\begin{array}{rrrr}\mathrm{C} & -7.86550900 & 1.55732200 & 0.40899300 \\ \mathrm{H} & -6.74035900 & 3.34612400 & 0.02224200 \\ \mathrm{C} & -9.05309200 & -1.97577000 & 0.73609600 \\ \mathrm{H} & -7.92445800 & -3.77428800 & 0.25734800 \\ \mathrm{H} & -9.91305300 & -0.04203600 & 1.10731300 \\ \mathrm{H} & -8.76190500 & 2.08457000 & 0.74756400 \\ \mathrm{H} & -9.94805300 & -2.52373200 & 1.04165400 \\ \mathrm{C} & 1.97685100 & 2.36353300 & -3.17327500 \\ \mathrm{H} & 2.13660900 & 3.30234800 & -3.72986100 \\ \mathrm{H} & 2.91541600 & 1.78897600 & -3.19697900 \\ \mathrm{H} & 1.76841700 & 2.61976900 & -2.12506000 \\ \mathrm{C} & 0.84629700 & 1.54430600 & -3.79701400 \\ \mathrm{O} & 0.72563900 & 0.28524000 & -3.15335800 \\ \mathrm{C} & -0.45727700 & 2.35145500 & -3.99160800 \\ \mathrm{H} & -0.25071000 & 3.02485100 & -4.84173800 \\ \mathrm{H} & -1.24427600 & 1.66843600 & -4.35565400 \\ \mathrm{C} & -1.00133800 & 3.23154300 & -2.85242500 \\ \mathrm{H} & -0.17159900 & 3.63344700 & -2.24562200 \\ \mathrm{H} & -1.50357200 & 4.10870800 & -3.28445600 \\ \mathrm{C} & -2.01180600 & 2.62866500 & -1.87001600 \\ \mathrm{O} & -2.95528200 & 3.34291800 & -1.50479500 \\ \mathrm{O} & -1.83197600 & 1.43643500 & -1.42474200 \\ \mathrm{H} & 1.16961300 & 1.28840900 & -4.82735700 \\ \mathrm{H} & -0.55906500 & -0.36266600 & -3.34597900 \\ \mathrm{H} & -1.35804500 & -0.75408600 & -3.06051000\end{array}$

\section{IntMR1}

$\begin{array}{rrrr}\text { Ir } & 0.90798500 & -0.12500900 & 0.85756000 \\ \text { P } & -0.92608000 & 0.30066300 & -0.49315200 \\ \text { O } & 2.45083300 & -3.08307100 & -1.84273700 \\ \text { N } & 1.95070000 & -1.31356100 & -0.56899500 \\ \text { C } & 3.18805100 & -0.91200500 & -1.30309300 \\ \text { C } & 3.30452200 & -2.03316600 & -2.35836700 \\ \text { C } & 1.68153100 & -2.52814200 & -0.89360700 \\ \text { C } & 0.61469000 & -3.36576200 & -0.30258900 \\ \text { C } & 0.87453400 & -4.05852900 & 0.89129800 \\ \text { C } & -0.11163700 & -4.86036700 & 1.46894800 \\ \text { C } & -1.36692100 & -4.97920700 & 0.86147000 \\ \text { C } & -1.62687100 & -4.28259700 & -0.31953300 \\ \text { C } & -2.86949000 & -4.32961200 & -1.17731800 \\ \text { C } & -2.65878700 & -3.17456200 & -2.17574600 \\ \text { C } & -1.11124600 & -2.95571100 & -2.26444700 \\ \text { C } & -0.64661500 & -3.46121300 & -0.90245200 \\ \text { C } & -0.50401000 & -3.90939900 & -3.34621700\end{array}$




\begin{tabular}{|c|c|c|c|}
\hline $\mathrm{C}$ & -0.64111800 & -3.15671400 & -4.66793900 \\
\hline $\mathrm{C}$ & -0.57616000 & -1.71240200 & -4.23726000 \\
\hline $\mathrm{C}$ & -0.38680400 & -0.64028000 & -5.10819500 \\
\hline $\mathrm{C}$ & -0.42868600 & 0.65035800 & -4.59489400 \\
\hline $\mathrm{C}$ & -0.63600000 & 0.84035000 & -3.22788300 \\
\hline $\mathrm{C}$ & -0.79977400 & -0.22098100 & -2.31209100 \\
\hline $\mathrm{C}$ & -0.78146000 & -1.54923500 & -2.84440500 \\
\hline $\mathrm{C}$ & -2.59842500 & -0.32588000 & -0.00600100 \\
\hline $\mathrm{C}$ & -3.68330900 & -0.07971400 & -0.86638700 \\
\hline $\mathrm{C}$ & -4.98113400 & -0.48695200 & -0.53256900 \\
\hline $\mathrm{C}$ & -5.15328400 & -1.14830100 & 0.69631500 \\
\hline $\mathrm{C}$ & -4.09758700 & -1.41597600 & 1. 58045000 \\
\hline $\mathrm{C}$ & -2.80931400 & -0.98957800 & 1. 20741800 \\
\hline $\mathrm{C}$ & -4.38639300 & -2.13500000 & 2. 91586300 \\
\hline $\mathrm{C}$ & -3.09904200 & -2.45435800 & 3. 69597500 \\
\hline $\mathrm{C}$ & -5.12480200 & -3.46713900 & 2. 64884600 \\
\hline $\mathrm{C}$ & -5.27626100 & -1.22363500 & 3. 79346000 \\
\hline $\mathrm{C}$ & -6.19434800 & -0.25749000 & -1.45868200 \\
\hline $\mathrm{C}$ & -5.81401100 & 0.51797500 & -2.73336300 \\
\hline $\mathrm{C}$ & -6.77730800 & -1.62587200 & -1.88257100 \\
\hline $\mathrm{C}$ & -7.27851100 & 0.54962700 & -0.70785300 \\
\hline $\mathrm{C}$ & -1.19171800 & 2. 15285300 & -0.51369100 \\
\hline $\mathrm{C}$ & -0.13508900 & 2. 99575500 & -0.91342200 \\
\hline $\mathrm{C}$ & -0.23628200 & 4. 38651300 & -0.84066100 \\
\hline $\mathrm{C}$ & -1.43051900 & 4. 93018200 & -0.32741800 \\
\hline $\mathrm{C}$ & -2.49481200 & 4. 13396800 & 0.10109700 \\
\hline $\mathrm{C}$ & -2.35354700 & 2. 73504700 & -0.00253700 \\
\hline $\mathrm{C}$ & -3.79845300 & 4. 72733200 & 0.67449900 \\
\hline $\mathrm{C}$ & -4.03306700 & 4. 18061500 & 2. 10122600 \\
\hline $\mathrm{C}$ & -3.75718300 & 6.26444100 & 0.75261000 \\
\hline $\mathrm{C}$ & -4.98399900 & 4. 32338800 & -0.23203500 \\
\hline $\mathrm{C}$ & 0.90238400 & 5.33069500 & -1.27863600 \\
\hline $\mathrm{C}$ & 2. 12144100 & 4. 56483000 & -1.82414300 \\
\hline $\mathrm{C}$ & 1. 36531700 & 6. 16925800 & -0.06446000 \\
\hline $\mathrm{C}$ & 0.39132000 & 6.27485300 & -2.39158700 \\
\hline $\mathrm{H}$ & 0.15249200 & -1.38432600 & 1. 35804200 \\
\hline $\mathrm{H}$ & 3. 00739300 & 0.06391300 & -1.77808600 \\
\hline $\mathrm{H}$ & 2. 91698900 & -1.74205200 & -3.34768700 \\
\hline $\mathrm{H}$ & 4. 31941100 & -2.43740000 & -2.46141500 \\
\hline $\mathrm{H}$ & 1.85046200 & -3.95565600 & 1. 37062300 \\
\hline $\mathrm{H}$ & 0.10278000 & -5.39456600 & 2. 39753400 \\
\hline $\mathrm{H}$ & -2.13429400 & -5.61978100 & 1. 30479400 \\
\hline $\mathrm{H}$ & -2.93494700 & -5.30731200 & -1.68832500 \\
\hline $\mathrm{H}$ & -3.79699200 & -4.21708200 & -0.59434600 \\
\hline $\mathrm{H}$ & -3.12911900 & -2.26614700 & -1.78472000 \\
\hline
\end{tabular}




\begin{tabular}{|c|c|c|c|}
\hline H & -3.09768400 & -3.37144400 & -3.16551200 \\
\hline $\mathrm{H}$ & 0.55992400 & -4.07258700 & -3.12269500 \\
\hline $\mathrm{H}$ & -0.99731300 & -4.89282200 & -3.33775400 \\
\hline $\mathrm{H}$ & 0.14056400 & -3.41719400 & -5.39946900 \\
\hline H & -1.61008800 & -3.35979500 & -5.15899000 \\
\hline $\mathrm{H}$ & -0.22752800 & -0.81983700 & -6.17503900 \\
\hline $\mathrm{H}$ & -0.30596900 & 1.51849200 & -5.24743400 \\
\hline $\mathrm{H}$ & -0.67980700 & 1.86488100 & -2.86872100 \\
\hline $\mathrm{H}$ & -3.49802500 & 0.43466000 & -1.80860200 \\
\hline H & -6.15829400 & -1.47046300 & 0.97480400 \\
\hline $\mathrm{H}$ & -1.95673000 & -1.16774000 & 1. 85880500 \\
\hline $\mathrm{H}$ & -2.54986400 & -1.54392000 & 3. 97713600 \\
\hline H & -2.41762700 & -3.09667200 & 3. 11708600 \\
\hline $\mathrm{H}$ & -3.35151500 & -2.98646600 & 4. 62673500 \\
\hline H & -6.09157700 & -3.31799300 & 2. 14469300 \\
\hline $\mathrm{H}$ & -5.32698700 & -3.98720600 & 3. 59934800 \\
\hline H & -4.51606500 & -4.13678700 & 2. 02063100 \\
\hline $\mathrm{H}$ & -4.77021600 & -0.26962600 & 4. 00992500 \\
\hline $\mathrm{H}$ & -5.50080400 & -1.71500800 & 4. 75447100 \\
\hline $\mathrm{H}$ & -6.23512300 & -0.99233500 & 3. 30389800 \\
\hline $\mathrm{H}$ & -5.40561400 & 1. 51433700 & -2.50366000 \\
\hline H & -6.70786000 & 0.66353700 & -3.35999400 \\
\hline $\mathrm{H}$ & -5.07167100 & -0.02365100 & -3.33981100 \\
\hline $\mathrm{H}$ & -6.02933500 & -2.22203100 & -2.42932300 \\
\hline $\mathrm{H}$ & -7.64795200 & -1.48450200 & -2.54393600 \\
\hline $\mathrm{H}$ & -7.11016700 & -2.21756900 & -1.01611200 \\
\hline $\mathrm{H}$ & -6.89450400 & 1. 53320600 & -0.39489100 \\
\hline $\mathrm{H}$ & -7.63423000 & 0.02665000 & 0.19268300 \\
\hline $\mathrm{H}$ & -8.15140300 & 0.71796800 & -1.35956500 \\
\hline $\mathrm{H}$ & 0.78368100 & 2. 55129100 & -1.28804400 \\
\hline $\mathrm{H}$ & -1.51584400 & 6.01346400 & -0.25637900 \\
\hline $\mathrm{H}$ & -3.16484900 & 2. 09614300 & 0.34024900 \\
\hline $\mathrm{H}$ & -4.12638700 & 3. 08442400 & 2. 11323300 \\
\hline $\mathrm{H}$ & -4.96173200 & 4. 59832700 & 2. 52398400 \\
\hline $\mathrm{H}$ & -3.20078100 & 4. 45070200 & 2. 76952100 \\
\hline $\mathrm{H}$ & -4.70340400 & 6.63991800 & 1. 17289700 \\
\hline $\mathrm{H}$ & -3.63221400 & 6.72650000 & -0.23929000 \\
\hline $\mathrm{H}$ & -2.94316400 & 6.62144000 & 1. 40263800 \\
\hline $\mathrm{H}$ & -5.09589300 & 3. 23026800 & -0.29172900 \\
\hline $\mathrm{H}$ & -4.84690500 & 4. 70416100 & -1.25685800 \\
\hline $\mathrm{H}$ & -5.92857300 & 4. 73496000 & 0.16067300 \\
\hline $\mathrm{H}$ & 1. 86675000 & 3. 95811000 & -2.70771700 \\
\hline $\mathrm{H}$ & 2. 56490600 & 3.90085900 & -1.06622600 \\
\hline$\pi$ & 2. 90294600 & 5. 27805700 & -2.12940900 \\
\hline . & 0.54953500 & 6. 78383800 & 0.34559300 \\
\hline
\end{tabular}




$\begin{array}{rrrr}\mathrm{H} & 2.18108600 & 6.85092700 & -0.35658600 \\ \mathrm{H} & 1.73522400 & 5.52078400 & 0.74510100 \\ \mathrm{H} & -0.45559000 & 6.89183400 & -2.05443300 \\ \mathrm{H} & 0.05893200 & 5.70432900 & -3.27381900 \\ \mathrm{H} & 1.19361800 & 6.95948700 & -2.71215200 \\ \mathrm{C} & 4.37546700 & -0.83042700 & -0.33912900 \\ \mathrm{H} & 4.57351000 & -1.85019700 & 0.03999000 \\ \mathrm{H} & 4.04803600 & -0.28548800 & 0.55824600 \\ \mathrm{C} & 5.66120100 & -0.24289000 & -0.87565800 \\ \mathrm{C} & 6.76243700 & -0.07556300 & 0.04079200 \\ \mathrm{C} & 5.83066400 & 0.13057200 & -2.19845300 \\ \mathrm{C} & 6.66113000 & -0.39730200 & 1.42734900 \\ \mathrm{C} & 8.00915000 & 0.44410000 & -0.45098800 \\ \mathrm{C} & 7.05930900 & 0.65474200 & -2.67695500 \\ \mathrm{H} & 5.00964400 & 0.02945500 & -2.91234800 \\ \mathrm{C} & 7.74177500 & -0.22641600 & 2.27111100 \\ \mathrm{H} & 5.72565200 & -0.76179600 & 1.86020100 \\ \mathrm{C} & 9.10017100 & 0.60070400 & 0.44838700 \\ \mathrm{C} & 8.12795200 & 0.80095000 & -1.82483500 \\ \mathrm{H} & 7.14844700 & 0.93637400 & -3.72947700 \\ \mathrm{C} & & & \\ \mathrm{H} & & & \end{array}$




\section{TSMR1}

\begin{tabular}{|c|c|c|c|}
\hline Ir & -0.87924500 & -0.20882000 & -0.86469800 \\
\hline $\mathrm{P}$ & 0.92948000 & 0.28153100 & 0.49159400 \\
\hline 0 & -2.37245500 & -2.97224300 & 1. 96943300 \\
\hline $\mathrm{N}$ & -1.85864700 & -1.30899000 & 0.57557100 \\
\hline $\mathrm{C}$ & -3.05519000 & -0.81127800 & 1. 31774500 \\
\hline $\mathrm{C}$ & -3.16281300 & -1.85403200 & 2. 45001000 \\
\hline $\mathrm{C}$ & -1.62004800 & -2.51419600 & 0.96128900 \\
\hline $\mathrm{C}$ & -0.59759800 & -3.41495700 & 0.38686600 \\
\hline $\mathrm{C}$ & -0.89868600 & -4.14482100 & -0.77371600 \\
\hline $\mathrm{C}$ & 0.06711900 & -4.96808000 & -1.35521600 \\
\hline $\mathrm{C}$ & 1. 34344700 & -5.06625900 & -0.78878700 \\
\hline $\mathrm{C}$ & 1. 64125100 & -4.33675600 & 0.36326500 \\
\hline $\mathrm{C}$ & 2. 91162500 & -4.35761300 & 1. 18110000 \\
\hline $\mathrm{C}$ & 2. 72374200 & -3.18623400 & 2. 16629400 \\
\hline $\mathrm{C}$ & 1. 17901900 & -2.97117500 & 2. 29334200 \\
\hline $\mathrm{C}$ & 0.67860000 & -3.50199300 & 0.95445400 \\
\hline $\mathrm{C}$ & 0.60483800 & -3.91458800 & 3. 40240600 \\
\hline $\mathrm{C}$ & 0.77051400 & -3.14425200 & 4. 70984100 \\
\hline $\mathrm{C}$ & 0.66677700 & -1.70805300 & 4. 26092200 \\
\hline $\mathrm{C}$ & 0.45561100 & -0.63002300 & 5. 11887000 \\
\hline $\mathrm{C}$ & 0.43972400 & 0.65308500 & 4. 58527700 \\
\hline $\mathrm{C}$ & 0.62375200 & 0.82913300 & 3. 21359900 \\
\hline $\mathrm{C}$ & 0.82155900 & -0.23945800 & 2. 31216600 \\
\hline $\mathrm{C}$ & 0.84658500 & -1.56035600 & 2. 86215300 \\
\hline $\mathrm{C}$ & 2. 58994000 & -0.33298000 & -0.04488400 \\
\hline $\mathrm{C}$ & 3. 70734500 & -0.14488400 & 0.78698300 \\
\hline $\mathrm{C}$ & 4. 98826800 & -0.54299600 & 0.38028900 \\
\hline $\mathrm{C}$ & 5. 10964600 & -1.13154800 & -0.89100300 \\
\hline $\mathrm{C}$ & 4. 01853100 & -1.33761500 & -1.74865500 \\
\hline $\mathrm{C}$ & 2. 75079900 & -0.92711800 & -1.30108100 \\
\hline $\mathrm{C}$ & 4. 24071000 & -1.99044800 & -3.12885600 \\
\hline $\mathrm{C}$ & 2. 92253700 & -2.17576800 & -3.90130300 \\
\hline $\mathrm{C}$ & 4. 88962500 & -3.38217400 & -2.94706600 \\
\hline $\mathrm{C}$ & 5. 17708900 & -1.09350800 & -3.97174400 \\
\hline $\mathrm{C}$ & 6. 23839100 & -0.37234300 & 1. 26991700 \\
\hline $\mathrm{C}$ & 5.90542800 & 0.28584000 & 2. 62119300 \\
\hline $\mathrm{C}$ & 6.85997700 & -1.76043200 & 1. 55102600 \\
\hline $\mathrm{C}$ & 7. 27502700 & 0.51400100 & 0.54163300 \\
\hline $\mathrm{C}$ & 1. 18518500 & 2. 13495500 & 0.53227700 \\
\hline $\mathrm{C}$ & 0.07860400 & 2. 95004000 & 0.84254500 \\
\hline $\mathrm{C}$ & 0.16381900 & 4. 34325400 & 0.81447400 \\
\hline $\mathrm{C}$ & 1. 39470600 & 4. 91751200 & 0.43723600 \\
\hline $\mathrm{C}$ & 2. 51011500 & 4. 14762400 & 0.09806100 \\
\hline $\mathrm{C}$ & 2. 38443900 & 2. 74411800 & 0.15765700 \\
\hline
\end{tabular}




\begin{tabular}{|c|c|c|c|}
\hline $\mathrm{C}$ & 3. 85151900 & 4. 77290900 & -0.33857700 \\
\hline $\mathrm{C}$ & 4. 21737800 & 4. 27044200 & -1.75463500 \\
\hline $\mathrm{C}$ & 3. 79552100 & 6.31085900 & -0.37593400 \\
\hline $\mathrm{C}$ & 4. 95908000 & 4. 35637700 & 0.65631400 \\
\hline $\mathrm{C}$ & -1.03635100 & 5. 25407700 & 1. 14519600 \\
\hline $\mathrm{C}$ & -2.27267900 & 4. 45067800 & 1. 58806000 \\
\hline $\mathrm{C}$ & -1.41569300 & 6.06887700 & -0.11375000 \\
\hline $\mathrm{C}$ & -0.65523600 & 6.22257400 & 2. 28837000 \\
\hline $\mathrm{H}$ & -0.11881800 & -1.53991700 & -1.27770400 \\
\hline $\mathrm{H}$ & -2.82341300 & 0.19125800 & 1. 70400500 \\
\hline $\mathrm{H}$ & -2.71575400 & -1.51521400 & 3. 39809800 \\
\hline $\mathrm{H}$ & -4.18528300 & -2.21079700 & 2. 62569100 \\
\hline $\mathrm{H}$ & -1.88489100 & -4.04350300 & -1.23154400 \\
\hline $\mathrm{H}$ & -0.17741100 & -5.53037700 & -2.25949300 \\
\hline $\mathrm{H}$ & 2. 09726400 & -5.71754100 & -1.24015700 \\
\hline $\mathrm{H}$ & 3. 00511300 & -5.32520600 & 1. 70671400 \\
\hline $\mathrm{H}$ & 3.81789200 & -4.24616200 & 0.56487800 \\
\hline $\mathrm{H}$ & 3. 17988300 & -2.28332200 & 1. 74732200 \\
\hline $\mathrm{H}$ & 3. 19039300 & -3.36548000 & 3. 14677900 \\
\hline $\mathrm{H}$ & -0.46415700 & -4.08298400 & 3. 20774500 \\
\hline $\mathrm{H}$ & 1. 10206600 & -4.89594900 & 3. 39271700 \\
\hline $\mathrm{H}$ & 0.01647600 & -3.40748000 & 5. 46873500 \\
\hline $\mathrm{H}$ & 1. 75810700 & -3.32489800 & 5. 17181100 \\
\hline $\mathrm{H}$ & 0.31764700 & -0.79897600 & 6. 19040500 \\
\hline $\mathrm{H}$ & 0.28913300 & 1. 52605700 & 5. 22536700 \\
\hline $\mathrm{H}$ & 0.61774700 & 1.84863500 & 2. 83754300 \\
\hline $\mathrm{H}$ & 3.56278600 & 0.31526700 & 1. 76390200 \\
\hline $\mathrm{H}$ & 6. 10041600 & -1.44647000 & -1.22438500 \\
\hline $\mathrm{H}$ & 1.86604300 & -1.07199700 & -1.91777700 \\
\hline $\mathrm{H}$ & 2. 42593300 & -1.21484500 & -4.10463400 \\
\hline $\mathrm{H}$ & 2. 21208400 & -2.81530500 & -3.35543100 \\
\hline $\mathrm{H}$ & 3. 12453700 & -2.65350300 & -4.87281000 \\
\hline $\mathrm{H}$ & 5. 86503200 & -3.32436500 & -2.43985200 \\
\hline $\mathrm{H}$ & 5. 05475100 & -3.85851600 & -3.92724200 \\
\hline $\mathrm{H}$ & 4. 23883600 & -4.04424700 & -2.35415400 \\
\hline $\mathrm{H}$ & 4. 73863400 & -0.09363300 & -4.11831200 \\
\hline $\mathrm{H}$ & 5. 34508100 & -1.53997300 & -4.96560100 \\
\hline $\mathrm{H}$ & 6.16105000 & -0.96210100 & -3.49561400 \\
\hline $\mathrm{H}$ & 5. 47996600 & 1. 29348200 & 2. 49600000 \\
\hline $\mathrm{H}$ & 6.82331300 & 0.38790800 & 3. 22119700 \\
\hline $\mathrm{H}$ & 5. 19297200 & -0.31481300 & 3. 20777300 \\
\hline $\mathrm{H}$ & 6. 14439100 & -2.41530500 & 2. 07334400 \\
\hline $\mathrm{H}$ & 7. 75465900 & -1.65926600 & 2. 18709300 \\
\hline $\mathrm{H}$ & 7. 16661600 & -2.26980300 & 0.62477500 \\
\hline $\mathrm{H}$ & 6. 86589600 & 1. 51606700 & 0.33795800 \\
\hline
\end{tabular}




\begin{tabular}{|c|c|c|c|}
\hline H & 7. 58709200 & 0.07849700 & -0.41976500 \\
\hline $\mathrm{H}$ & 8. 17901200 & 0.63620300 & 1. 16056600 \\
\hline $\mathrm{H}$ & -0.86750300 & 2. 47228500 & 1. 08426900 \\
\hline $\mathrm{H}$ & 1. 46772400 & 6.00356600 & 0.39878700 \\
\hline H & 3. 23768700 & 2. 12605100 & -0.11407000 \\
\hline $\mathrm{H}$ & 4. 33125200 & 3.17646300 & -1.78734400 \\
\hline $\mathrm{H}$ & 5. 17027700 & 4. 71490800 & -2.08641400 \\
\hline $\mathrm{H}$ & 3. 43989500 & 4. 54788300 & -2.48407500 \\
\hline $\mathrm{H}$ & 4. 77157200 & 6. 71087400 & -0.69260300 \\
\hline H & 3. 56976400 & 6.73970300 & 0.61294900 \\
\hline $\mathrm{H}$ & 3. 04116100 & 6.67825000 & -1.08908200 \\
\hline H & 5. 07832200 & 3. 26320000 & 0.69862500 \\
\hline H & 4. 72913000 & 4. 70868400 & 1. 67456700 \\
\hline $\mathrm{H}$ & 5. 92939800 & 4. 78720000 & 0.35841700 \\
\hline H & -2.07420100 & 3. 86202300 & 2. 49800100 \\
\hline $\mathrm{H}$ & -2.62145200 & 3. 76167700 & 0.80384800 \\
\hline $\mathrm{H}$ & -3.10169000 & 5. 13912300 & 1. 81543900 \\
\hline $\mathrm{H}$ & -0.58235300 & 6.69847900 & -0.46172700 \\
\hline $\mathrm{H}$ & -2.26953000 & 6.73271600 & 0.10029000 \\
\hline $\mathrm{H}$ & -1.70196700 & 5. 40188800 & -0.94190600 \\
\hline $\mathrm{H}$ & 0.19410600 & 6.86885800 & 2. 01920500 \\
\hline H & -0.37980700 & 5. 66981700 & 3. 20105400 \\
\hline $\mathrm{H}$ & -1.50564300 & 6. 87979900 & 2. 53377900 \\
\hline $\mathrm{C}$ & -4.26814800 & -0.76897600 & 0.38599300 \\
\hline $\mathrm{H}$ & -4.47284800 & -1.80263500 & 0.05370300 \\
\hline $\mathrm{H}$ & -3.96373800 & -0.25666900 & -0.53736600 \\
\hline $\mathrm{C}$ & -5.54161500 & -0.16245300 & 0.92701100 \\
\hline $\mathrm{C}$ & -6.68896800 & -0.11997200 & 0.05415900 \\
\hline $\mathrm{C}$ & -5.65879900 & 0.34745700 & 2. 20884600 \\
\hline $\mathrm{C}$ & -6.64280100 & -0.58574300 & -1.29390700 \\
\hline $\mathrm{C}$ & -7.92597700 & 0.41904900 & 0.54827800 \\
\hline $\mathrm{C}$ & -6.87862700 & 0.89088600 & 2. 68877100 \\
\hline $\mathrm{H}$ & -4.80113200 & 0.34460200 & 2. 88598000 \\
\hline $\mathrm{C}$ & -7.76541900 & -0.53466300 & -2.09734900 \\
\hline $\mathrm{H}$ & -5.71609900 & -0.97107200 & -1.72615200 \\
\hline $\mathrm{C}$ & -9.06173400 & 0.45006800 & -0.30787700 \\
\hline $\mathrm{C}$ & -7.99021300 & 0.91916900 & 1. 88050400 \\
\hline $\mathrm{H}$ & -6.92650700 & 1. 28230200 & 3. 70838700 \\
\hline $\mathrm{C}$ & -8.98720500 & -0.01940900 & -1.60239600 \\
\hline $\mathrm{H}$ & -7.70056300 & -0.89101200 & -3.12837800 \\
\hline $\mathrm{H}$ & -9.99881100 & 0.85901300 & 0.08083800 \\
\hline $\mathrm{H}$ & -8.93583400 & 1. 32951500 & 2. 24550800 \\
\hline $\mathrm{H}$ & -9.86737500 & 0.01222700 & -2.25015600 \\
\hline $\mathrm{C}$ & 0.39223300 & 2. 60682600 & -4.10635800 \\
\hline 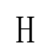 & 0.66089200 & 2. 61397800 & -5.17490500 \\
\hline
\end{tabular}




$\begin{array}{lrrr}\mathrm{H} & 1.25629700 & 2.95705500 & -3.52245000 \\ \mathrm{H} & -0.43367400 & 3.32137200 & -3.95466900 \\ \mathrm{C} & -0.02114000 & 1.20693800 & -3.64682300 \\ \mathrm{O} & -0.16888900 & 1.25997200 & -2.23556200 \\ \mathrm{C} & -1.23180900 & 0.69218400 & -4.43746500 \\ \mathrm{H} & -2.10563900 & 1.33259600 & -4.22236000 \\ \mathrm{H} & -0.99346900 & 0.86255100 & -5.50099300 \\ \mathrm{C} & -1.59829000 & -0.79798400 & -4.26101500 \\ \mathrm{H} & -1.91654800 & -1.22275200 & -5.22290900 \\ \mathrm{H} & -0.71251600 & -1.36371600 & -3.92193600 \\ \mathrm{C} & -2.74557500 & -1.03285100 & -3.27919000 \\ \mathrm{O} & -3.81603800 & -1.48849500 & -3.66075300 \\ \mathrm{O} & -2.54476600 & -0.68337900 & -2.04097900 \\ \mathrm{H} & 0.81907200 & 0.50991300 & -3.84410500 \\ \mathrm{H} & -1.15448000 & 1.62340100 & -1.57246400 \\ \mathrm{H} & -1.77368200 & 1.46102300 & -0.73257800\end{array}$

\section{IntNR}

Ir

$\mathrm{P}$

0

$\mathrm{N}$

C

C

C

C

C

C

C

C

C

C

C

C

C

C

C

C

C

C

C

C

C

C
$-0.42921400$

0. 87846600

$-2.76159400$

$-1.86838100$

$-3.09755200$

$-3.38069700$

$-1.84148000$

$-0.87624400$

$-1.10162200$

$-0.14205800$

1. 06215200

1. 28753500

2. 47790900

2. 25928200

0. 71869500

0. 32251500

0. 00918800

0. 06813200

0. 03101800

$-0.26819200$

$-0.22214600$

0. 12163700

0. 42031200

0. 36547700

2. 64860500

3. 51644500
$-0.43972000$

0.16285500

$-2.78246500$

$-1.26837600$

$-0.61769600$

$-1.51763900$

$-2.47669400$

$-3.50925800$

$-4.20665100$

$-5.09442200$

$-5.28004200$

$-4.58338500$

$-4.66809000$

$-3.48646900$

$-3.22081900$

$-3.69939500$

$-4.19055700$

$-3.48465900$

$-2.03074100$

$-0.99373000$

0. 31112300

0. 54563200

$-0.47781300$

$-1.82478900$

$-0.35819100$

-0. 22307000
$-1.42342800$

0. 31861600

1. 22395300

$-0.15296800$

0. 39129700

1. 60097900

0. 30079800

$-0.13514000$

$-1.33121700$

$-1.82167000$

$-1.13461600$

0. 05402900

0. 98115000

1. 94794800

1. 95114100

0. 56089400

2. 95592100

4. 30488700

3. 90937800

4. 79043000

4. 31869700

2. 98722600

2. 06090200

2. 54554500

0. 16996000

1. 26834000 


\begin{tabular}{|c|c|c|c|}
\hline $\mathrm{C}$ & 4. 86214700 & -0.60233100 & 1. 17381700 \\
\hline $\mathrm{C}$ & 5. 30352300 & -1.12845200 & -0.05431900 \\
\hline $\mathrm{C}$ & 4. 46375000 & -1.28628500 & -1.16657500 \\
\hline $\mathrm{C}$ & 3. 12312300 & -0.88256600 & -1.03748100 \\
\hline $\mathrm{C}$ & 5. 01770200 & -1.85804500 & -2.48897000 \\
\hline $\mathrm{C}$ & 3. 89044200 & -2.21336800 & -3.47742200 \\
\hline $\mathrm{C}$ & 5. 83392400 & -3.14237300 & -2.21917400 \\
\hline $\mathrm{C}$ & 5. 93616800 & -0.79872600 & -3.14214600 \\
\hline $\mathrm{C}$ & 5.84790300 & -0.48473700 & 2. 35562500 \\
\hline $\mathrm{C}$ & 5. 18126000 & 0.10064100 & 3. 61409900 \\
\hline $\mathrm{C}$ & 6. 39562900 & -1.88688300 & 2. 71019000 \\
\hline $\mathrm{C}$ & 7. 02393200 & 0.43743000 & 1. 95831800 \\
\hline $\mathrm{C}$ & 0.97967300 & 2. 01015100 & 0.55180200 \\
\hline $\mathrm{C}$ & -0.21519900 & 2. 73935900 & 0.72712800 \\
\hline $\mathrm{C}$ & -0.19556800 & 4. 11894000 & 0.95991500 \\
\hline $\mathrm{C}$ & 1. 05485600 & 4. 76969900 & 0.92001500 \\
\hline $\mathrm{C}$ & 2. 25732300 & 4. 09103400 & 0.70293700 \\
\hline $\mathrm{C}$ & 2. 19946300 & 2. 69249100 & 0.54200900 \\
\hline $\mathrm{C}$ & 3. 62265000 & 4. 80918600 & 0.65185200 \\
\hline $\mathrm{C}$ & 4. 28333000 & 4. 56090800 & -0.72384200 \\
\hline $\mathrm{C}$ & 3. 49049500 & 6.32941700 & 0.85372400 \\
\hline $\mathrm{C}$ & 4. 53785400 & 4. 25357100 & 1. 76730600 \\
\hline $\mathrm{C}$ & -1.46777800 & 4. 93710000 & 1. 26941500 \\
\hline $\mathrm{C}$ & -2.69305600 & 4. 03327900 & 1. 49691500 \\
\hline $\mathrm{C}$ & -1.77395100 & 5. 88328800 & 0.08442300 \\
\hline $\mathrm{C}$ & -1.24101000 & 5. 76977600 & 2. 55332000 \\
\hline $\mathrm{H}$ & 0.44509700 & -1.73051200 & -1.33441600 \\
\hline $\mathrm{H}$ & -2.86827300 & 0.41419300 & 0.68078300 \\
\hline $\mathrm{H}$ & -2.87850600 & -1.16913900 & 2.51735100 \\
\hline $\mathrm{H}$ & -4.44213900 & -1.69903400 & 1. 80125500 \\
\hline $\mathrm{H}$ & -2.01731000 & -4.02396600 & -1.89609600 \\
\hline $\mathrm{H}$ & -0.32796400 & -5.62767000 & -2.75686900 \\
\hline $\mathrm{H}$ & 1. 81861300 & -5.96657400 & -1.52498700 \\
\hline $\mathrm{H}$ & 2. 48056700 & -5.63809300 & 1. 51047600 \\
\hline $\mathrm{H}$ & 3. 43856100 & -4.60016200 & 0.44640000 \\
\hline $\mathrm{H}$ & 2. 77633600 & -2.60001300 & 1. 56368000 \\
\hline $\mathrm{H}$ & 2. 64431300 & -3.67721500 & 2.96135000 \\
\hline $\mathrm{H}$ & -1.04137800 & -4.30452000 & 2. 64924800 \\
\hline $\mathrm{H}$ & 0.46788100 & -5.19033800 & 2. 94821700 \\
\hline $\mathrm{H}$ & -0.75668400 & -3.76245900 & 4. 98016600 \\
\hline $\mathrm{H}$ & 1. 00709400 & -3.70593600 & 4. 84437500 \\
\hline $\mathrm{H}$ & -0.52568200 & -1.21299400 & 5. 83023700 \\
\hline $\mathrm{H}$ & -0.44406100 & 1. 15677600 & 4. 97433000 \\
\hline $\mathrm{H}$ & 0.16588600 & 1. 58187200 & 2. 66838700 \\
\hline $\mathrm{H}$ & 3. 12492300 & 0.16868500 & 2. 20641600 \\
\hline
\end{tabular}




\begin{tabular}{|c|c|c|c|}
\hline H & 6.34855200 & -1.43101100 & -0.14245400 \\
\hline $\mathrm{H}$ & 2. 43332400 & -0.94851000 & -1.87790000 \\
\hline $\mathrm{H}$ & 3. 28518600 & -1.34071600 & -3.76214800 \\
\hline $\mathrm{H}$ & 3. 20379000 & -2.96365900 & -3.05471700 \\
\hline H & 4. 32479500 & -2.63410100 & -4.39823900 \\
\hline $\mathrm{H}$ & 6.71485400 & -2.95874600 & -1.58532600 \\
\hline $\mathrm{H}$ & 6. 19939600 & -3.56603300 & -3.16862600 \\
\hline $\mathrm{H}$ & 5. 21524400 & -3.90836500 & -1.72423900 \\
\hline $\mathrm{H}$ & 5. 37576200 & 0.12232900 & -3.36680900 \\
\hline H & 6.35628800 & -1.17979400 & -4.08782900 \\
\hline $\mathrm{H}$ & 6.77733900 & -0.52853600 & -2.48405200 \\
\hline H & 4. 79073400 & 1. 11549800 & 3. 44118200 \\
\hline H & 5. 91884200 & 0.16638300 & 4. 42942000 \\
\hline $\mathrm{H}$ & 4. 35057400 & -0.52792000 & 3. 97073900 \\
\hline H & 5.58002500 & -2.56832600 & 3. 00036900 \\
\hline $\mathrm{H}$ & 7. 10102700 & -1.82175400 & 3. 55498500 \\
\hline $\mathrm{H}$ & 6.93118100 & -2.34620200 & 1. 86543300 \\
\hline $\mathrm{H}$ & 6.66697600 & 1. 44971200 & 1. 71107100 \\
\hline $\mathrm{H}$ & 7. 57180500 & 0.05288800 & 1. 08471900 \\
\hline $\mathrm{H}$ & 7. 74228200 & 0.52439900 & 2. 78997100 \\
\hline $\mathrm{H}$ & -1.16607900 & 2. 21087900 & 0.69553400 \\
\hline H & 1. 07592200 & 5. 84785000 & 1. 06900200 \\
\hline $\mathrm{H}$ & 3. 12254100 & 2. 13521600 & 0.39354200 \\
\hline $\mathrm{H}$ & 4. 44598300 & 3. 48971600 & -0.91600100 \\
\hline $\mathrm{H}$ & 5. 26387400 & 5.06240100 & -0.77455100 \\
\hline $\mathrm{H}$ & 3. 65680100 & 4. 95441400 & -1.53968900 \\
\hline $\mathrm{H}$ & 4. 48544100 & 6. 79933000 & 0.80482300 \\
\hline $\mathrm{H}$ & 3. 05650500 & 6.57932700 & 1. 83453500 \\
\hline $\mathrm{H}$ & 2. 86721400 & 6. 79521200 & 0.07466100 \\
\hline $\mathrm{H}$ & 4. 71525900 & 3. 17369700 & 1. 65086300 \\
\hline $\mathrm{H}$ & 4. 09345500 & 4. 41644900 & 2. 76228400 \\
\hline $\mathrm{H}$ & 5.51910300 & 4. 75592200 & 1. 74688200 \\
\hline $\mathrm{H}$ & -2.53168200 & 3. 33091700 & 2. 33163600 \\
\hline $\mathrm{H}$ & -2.94867600 & 3. 46974700 & 0.58861100 \\
\hline $\mathrm{H}$ & -3.56549600 & 4. 65467800 & 1. 75463700 \\
\hline $\mathrm{H}$ & -0.91822100 & 6.53849000 & -0.14545100 \\
\hline $\mathrm{H}$ & -2.63289000 & 6.53000900 & 0.33042700 \\
\hline $\mathrm{H}$ & -2.03365200 & 5. 30243100 & -0.81321900 \\
\hline $\mathrm{H}$ & -0.43425400 & 6.51035600 & 2. 44184300 \\
\hline $\mathrm{H}$ & -0.98863600 & 5. 12324500 & 3. 40987400 \\
\hline $\mathrm{H}$ & -2.15904600 & 6.32390500 & 2. 80750300 \\
\hline $\mathrm{C}$ & -4.21196900 & -0.59827500 & -0.68092000 \\
\hline $\mathrm{H}$ & -4.42206200 & -1.63249500 & -0.99450600 \\
\hline H & -3.78173100 & -0.06625300 & -1.53739700 \\
\hline 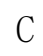 & -5.47129300 & 0.12208000 & -0.24000800 \\
\hline
\end{tabular}




$\begin{array}{lrrr}\mathrm{C} & -6.61813200 & -0.56966300 & 0.28580800 \\ \mathrm{C} & -5.50446400 & 1.50589300 & -0.35066200 \\ \mathrm{C} & -6.70037500 & -1.99086500 & 0.39805400 \\ \mathrm{C} & -7.76103300 & 0.19800500 & 0.71080900 \\ \mathrm{C} & -6.63206300 & 2.25103300 & 0.06975000 \\ \mathrm{H} & -4.64850000 & 2.03475200 & -0.78748700 \\ \mathrm{C} & -7.82253600 & -2.61410200 & 0.90571000 \\ \mathrm{H} & -5.86255300 & -2.60811000 & 0.07034600 \\ \mathrm{C} & -8.90100000 & -0.47728000 & 1.23249200 \\ \mathrm{C} & -7.73456600 & 1.61465900 & 0.59541900 \\ \mathrm{H} & -6.61818900 & 3.33904900 & -0.03351800 \\ \mathrm{C} & -8.93666200 & -1.85116900 & 1.33257500 \\ \mathrm{H} & -7.85322800 & -3.70471100 & 0.97422900 \\ \mathrm{H} & -9.75831700 & 0.12197100 & 1.55227000 \\ \mathrm{H} & -8.60820300 & 2.18570500 & 0.92221800 \\ \mathrm{H} & -9.82061900 & -2.35338400 & 1.73376300 \\ \mathrm{C} & 2.18498200 & 2.03273300 & -3.08218000 \\ \mathrm{H} & 2.43331700 & 2.93041100 & -3.67291500 \\ \mathrm{H} & 3.08413400 & 1.40108700 & -3.02677800 \\ \mathrm{H} & 1.94022300 & 2.35615900 & -2.06192100 \\ \mathrm{C} & -1.03143500 & 1.25653200 & -3.72159900 \\ \mathrm{O} & -2.77028400 & 0.04112200 & -3.05300000 \\ \mathrm{C} & -0.18915500 & 2.15716200 & -4.02370100 \\ \mathrm{H} & -2.14560900 & 2.84373500 & -4.82140900 \\ \mathrm{H} & -0.97382600 & 1.53708400 & -4.48967100 \\ \mathrm{C} & -0.80034700 & 3.02215800 & -2.90722500 \\ \mathrm{H} & -0.02050200 & 3.34527100 & -2.19665200 \\ \mathrm{H} & -1.20483500 & 3.94803700 & -3.34106400 \\ \mathrm{C} & -1.94195000 & 2.42345500 & -2.07645500 \\ \mathrm{O} & -2.87474000 & 3.16649300 & -1.74689100 \\ \mathrm{H} & -1.88701900 & 1.18103800 & -1.74117400 \\ \mathrm{H} & 1.39187000 & 0.94215300 & -4.72454700 \\ \mathrm{H} & -1.27756700 & -1.33144500 & -3.33174600 \\ \mathrm{H} & -2.46209500 & -0.87314800 & -3.70521200 \\ \mathrm{C} & -0.98677500 & -4.02782400 \\ \mathrm{H} & -1.28524800 & -5.08696800 \\ \mathrm{H} & -1.54356900 & -3.57763000 \\ \mathrm{H} & -385600 & 0.08881200 & -3.96127900\end{array}$

\section{TSNR}

Ir
P
0
N

$\begin{array}{rrr}-0.45218400 & -0.43231600 & -1.44136100 \\ 0.86755600 & 0.14485800 & 0.34648400 \\ -2.75304600 & -2.80278900 & 1.16352500 \\ -1.85982100 & -1.27796000 & -0.19676100\end{array}$




\begin{tabular}{|c|c|c|c|}
\hline $\mathrm{C}$ & -3.08744900 & -0.62656700 & 0.35352100 \\
\hline $\mathrm{C}$ & -3.37549400 & -1.54220500 & 1. 55020100 \\
\hline $\mathrm{C}$ & -1.83028900 & -2.49069500 & 0.24710800 \\
\hline $\mathrm{C}$ & -0.85524500 & -3.51453100 & -0.18504400 \\
\hline $\mathrm{C}$ & -1.05425600 & -4.19494200 & -1.39568400 \\
\hline $\mathrm{C}$ & -0.08245000 & -5.07403000 & -1.87702500 \\
\hline $\mathrm{C}$ & 1. 10663500 & -5.26760100 & -1.16605400 \\
\hline $\mathrm{C}$ & 1. 30545200 & -4.58701700 & 0.03658100 \\
\hline $\mathrm{C}$ & 2. 47460300 & -4.68616600 & 0.98898300 \\
\hline $\mathrm{C}$ & 2. 23557400 & -3.51733800 & 1. 96591700 \\
\hline $\mathrm{C}$ & 0.69521800 & -3.25017000 & 1. 93979800 \\
\hline $\mathrm{C}$ & 0.32882600 & -3.71053100 & 0.53474900 \\
\hline $\mathrm{C}$ & -0.03661300 & -4.22978200 & 2. 91830200 \\
\hline $\mathrm{C}$ & 0.00318500 & -3.54324200 & 4. 27821000 \\
\hline $\mathrm{C}$ & -0.01390600 & -2.08322800 & 3. 90416500 \\
\hline $\mathrm{C}$ & -0.30939400 & -1.05570300 & 4. 79743100 \\
\hline $\mathrm{C}$ & -0.23742600 & 0.25581800 & 4. 34724700 \\
\hline $\mathrm{C}$ & 0.12272100 & 0.50617800 & 3. 02313400 \\
\hline $\mathrm{C}$ & 0.41357600 & -0.50743400 & 2. 08362400 \\
\hline $\mathrm{C}$ & 0.33898400 & -1.86051600 & 2. 54758400 \\
\hline $\mathrm{C}$ & 2. 63719400 & -0.38082500 & 0.18641000 \\
\hline $\mathrm{C}$ & 3.51267500 & -0.24406100 & 1. 27833100 \\
\hline $\mathrm{C}$ & 4. 85960500 & -0.61713400 & 1. 17531900 \\
\hline $\mathrm{C}$ & 5. 29652500 & -1.13767200 & -0.05653700 \\
\hline $\mathrm{C}$ & 4. 45016800 & -1.29965900 & -1.16320000 \\
\hline $\mathrm{C}$ & 3. 10824000 & -0.90468600 & -1.02291400 \\
\hline $\mathrm{C}$ & 4. 99987900 & -1.87300700 & -2.48669800 \\
\hline $\mathrm{C}$ & 3. 87402200 & -2.19231000 & -3.48848300 \\
\hline $\mathrm{C}$ & 5. 78048600 & -3.17982200 & -2.21776500 \\
\hline $\mathrm{C}$ & 5. 95061600 & -0.83325200 & -3.12481000 \\
\hline $\mathrm{C}$ & 5. 85167400 & -0.49734400 & 2. 35156100 \\
\hline $\mathrm{C}$ & 5. 18996800 & 0.08400300 & 3. 61449400 \\
\hline $\mathrm{C}$ & 6. 40597900 & -1.89828600 & 2. 70067200 \\
\hline $\mathrm{C}$ & 7. 02240600 & 0.42923300 & 1. 94894100 \\
\hline $\mathrm{C}$ & 0.98750900 & 1.99000000 & 0.58882300 \\
\hline $\mathrm{C}$ & -0.20044100 & 2. 72293900 & 0.79463900 \\
\hline $\mathrm{C}$ & -0.17173300 & 4. 10228300 & 1. 02638200 \\
\hline $\mathrm{C}$ & 1. 07857100 & 4. 75050800 & 0.95341600 \\
\hline $\mathrm{C}$ & 2. 27315300 & 4. 06922100 & 0.70386700 \\
\hline $\mathrm{C}$ & 2. 20803000 & 2. 67038300 & 0.54475200 \\
\hline $\mathrm{C}$ & 3. 63856800 & 4. 78446300 & 0.62184100 \\
\hline $\mathrm{C}$ & 4. 27713800 & 4. 52254100 & -0.76168200 \\
\hline $\mathrm{C}$ & 3.51241500 & 6.30697000 & 0.81072400 \\
\hline $\mathrm{C}$ & 4. 57099700 & 4. 23785200 & 1. 72750200 \\
\hline $\mathrm{C}$ & -1.43266300 & 4. 92250500 & 1. 37396200 \\
\hline
\end{tabular}




\begin{tabular}{|c|c|c|c|}
\hline $\mathrm{C}$ & -2.66126800 & 4. 02314600 & 1. 60082000 \\
\hline $\mathrm{C}$ & -1.75169100 & 5. 89807300 & 0.21671000 \\
\hline $\mathrm{C}$ & -1.17616200 & 5. 72333400 & 2. 67263500 \\
\hline $\mathrm{H}$ & 0.39640900 & -1.74007800 & -1.41678100 \\
\hline $\mathrm{H}$ & -2.84935200 & 0.39917200 & 0.65632500 \\
\hline $\mathrm{H}$ & -2.87925600 & -1.20459900 & 2. 47373800 \\
\hline $\mathrm{H}$ & -4.43801900 & -1.72679900 & 1. 74138600 \\
\hline $\mathrm{H}$ & -1.95660600 & -4.00406400 & -1.97885600 \\
\hline $\mathrm{H}$ & -0.24677200 & -5.59370700 & -2.82375800 \\
\hline $\mathrm{H}$ & 1. 87224800 & -5.94835900 & -1.54872300 \\
\hline $\mathrm{H}$ & 2. 46316000 & -5.66287400 & 1. 50572000 \\
\hline $\mathrm{H}$ & 3. 44753300 & -4.61375900 & 0.47740300 \\
\hline $\mathrm{H}$ & 2. 76013000 & -2.62578100 & 1. 60388500 \\
\hline $\mathrm{H}$ & 2. 59908900 & -3.72084700 & 2. 98473500 \\
\hline $\mathrm{H}$ & -1.08207800 & -4.33378500 & 2. 59152900 \\
\hline $\mathrm{H}$ & 0.41715500 & -5.23175400 & 2.90474700 \\
\hline $\mathrm{H}$ & -0.83628400 & -3.82354700 & 4. 93417000 \\
\hline $\mathrm{H}$ & 0.92998900 & -3.78059400 & 4. 83168000 \\
\hline $\mathrm{H}$ & -0.58220900 & -1.28708900 & 5. 83068800 \\
\hline $\mathrm{H}$ & -0.45254500 & 1. 09417400 & 5. 01442800 \\
\hline $\mathrm{H}$ & 0.18588800 & 1.54642900 & 2. 72033800 \\
\hline $\mathrm{H}$ & 3. 12551700 & 0.14729400 & 2. 21838400 \\
\hline $\mathrm{H}$ & 6.34279500 & -1.43388900 & -0.15223700 \\
\hline $\mathrm{H}$ & 2. 41439200 & -0.99041900 & -1.85644300 \\
\hline $\mathrm{H}$ & 3. 29671900 & -1.30085900 & -3.77357200 \\
\hline $\mathrm{H}$ & 3. 16305400 & -2.92789300 & -3.08080800 \\
\hline $\mathrm{H}$ & 4. 30659700 & -2.61620200 & -4.40851400 \\
\hline $\mathrm{H}$ & 6.65623500 & -3.02190200 & -1.57015100 \\
\hline $\mathrm{H}$ & 6. 14824500 & -3.60507800 & -3.16566600 \\
\hline $\mathrm{H}$ & 5. 13634200 & -3.93365100 & -1.73709200 \\
\hline $\mathrm{H}$ & 5. 41563400 & 0.10292200 & -3.35009600 \\
\hline $\mathrm{H}$ & 6. 37012400 & -1.21965400 & -4.06851500 \\
\hline $\mathrm{H}$ & 6.79219500 & -0.58717800 & -2.45809000 \\
\hline $\mathrm{H}$ & 4. 79510900 & 1. 09779300 & 3. 44519600 \\
\hline $\mathrm{H}$ & 5.93181000 & 0.15097800 & 4. 42580700 \\
\hline $\mathrm{H}$ & 4. 36344700 & -0.54792300 & 3. 97477300 \\
\hline $\mathrm{H}$ & 5.59428800 & -2.58316700 & 2. 99359300 \\
\hline $\mathrm{H}$ & 7. 11525200 & -1.83221500 & 3. 54210200 \\
\hline $\mathrm{H}$ & 6.93911900 & -2.35407300 & 1. 85244400 \\
\hline $\mathrm{H}$ & 6. 66088400 & 1. 44063500 & 1. 70484600 \\
\hline $\mathrm{H}$ & 7. 56725800 & 0.04751200 & 1. 07220800 \\
\hline $\mathrm{H}$ & 7. 74471800 & 0.51767900 & 2. 77694600 \\
\hline $\mathrm{H}$ & -1.15248800 & 2. 19646800 & 0.78334500 \\
\hline $\mathrm{H}$ & 1. 10573600 & 5. 82854800 & 1. 10282500 \\
\hline $\mathrm{H}$ & 3. 12630800 & 2. 11113300 & 0.37465700 \\
\hline
\end{tabular}




\begin{tabular}{|c|c|c|c|}
\hline H & 4. 43687600 & 3. 44948100 & -0.94609500 \\
\hline $\mathrm{H}$ & 5. 25712900 & 5.02255200 & -0.83298400 \\
\hline $\mathrm{H}$ & 3. 63814200 & 4. 90950900 & -1.57105700 \\
\hline $\mathrm{H}$ & 4. 50662900 & 6.77516000 & 0.73683300 \\
\hline $\mathrm{H}$ & 3. 09901200 & 6.56764400 & 1. 79751900 \\
\hline $\mathrm{H}$ & 2.87339900 & 6. 76535800 & 0.04001900 \\
\hline H & 4. 74362900 & 3. 15640800 & 1. 61860200 \\
\hline $\mathrm{H}$ & 4. 14280900 & 4. 41114100 & 2. 72776400 \\
\hline $\mathrm{H}$ & 5.55289400 & 4. 73774200 & 1. 68657200 \\
\hline H & -2.49342400 & 3. 30240500 & 2. 41835000 \\
\hline $\mathrm{H}$ & -2.93321500 & 3. 48020400 & 0.68460400 \\
\hline $\mathrm{H}$ & -3.52513200 & 4. 64539200 & 1. 88409600 \\
\hline $\mathrm{H}$ & -0.89581300 & 6.55305800 & -0.01326200 \\
\hline $\mathrm{H}$ & -2.60139500 & 6.54500100 & 0.49237200 \\
\hline $\mathrm{H}$ & -2.03136100 & 5. 33973600 & -0.68919700 \\
\hline $\mathrm{H}$ & -0.36027100 & 6.45456900 & 2.56524400 \\
\hline $\mathrm{H}$ & -0.92017800 & 5. 05423200 & 3. 51058400 \\
\hline $\mathrm{H}$ & -2.08258500 & 6.28398900 & 2. 95295400 \\
\hline $\mathrm{C}$ & -4.20125700 & -0.58223100 & -0.71708200 \\
\hline $\mathrm{H}$ & -4.41130100 & -1.60770100 & -1.05711900 \\
\hline $\mathrm{H}$ & -3.77641300 & -0.03121800 & -1.56352600 \\
\hline $\mathrm{C}$ & -5.45946600 & 0.12647000 & -0.25386700 \\
\hline $\mathrm{C}$ & -6.60614500 & -0.58006800 & 0.25230200 \\
\hline $\mathrm{C}$ & -5.49194900 & 1. 51294200 & -0.32320100 \\
\hline $\mathrm{C}$ & -6.68811500 & -2.00389300 & 0.32406400 \\
\hline $\mathrm{C}$ & -7.74880100 & 0.17498800 & 0.69991100 \\
\hline $\mathrm{C}$ & -6.61941100 & 2.24557600 & 0.11906600 \\
\hline $\mathrm{H}$ & -4.63598100 & 2. 05510200 & -0.74339200 \\
\hline $\mathrm{C}$ & -7.80992300 & -2.64155400 & 0.81425500 \\
\hline $\mathrm{H}$ & -5.85034800 & -2.61127200 & -0.02158000 \\
\hline $\mathrm{C}$ & -8.88855400 & -0.51511600 & 1. 20232600 \\
\hline $\mathrm{C}$ & -7.72212400 & 1. 59436400 & 0.62577700 \\
\hline $\mathrm{H}$ & -6.60518300 & 3. 33614200 & 0.04769300 \\
\hline $\mathrm{C}$ & -8.92405900 & -1.89130900 & 1. 26300700 \\
\hline $\mathrm{H}$ & -7.84039100 & -3.73368000 & 0.85152500 \\
\hline $\mathrm{H}$ & -9.74582800 & 0.07463700 & 1. 53941300 \\
\hline $\mathrm{H}$ & -8.59569500 & 2. 15578700 & 0.96903500 \\
\hline $\mathrm{H}$ & -9.80789700 & -2.40491500 & 1. 64979700 \\
\hline $\mathrm{C}$ & 2. 15128000 & 2. 07342700 & -3.13368700 \\
\hline $\mathrm{H}$ & 2. 39387100 & 3. 00703500 & -3.66611700 \\
\hline $\mathrm{H}$ & 3.04269400 & 1. 42886000 & -3.14560200 \\
\hline $\mathrm{H}$ & 1.93123000 & 2. 32414000 & -2.08749100 \\
\hline $\mathrm{C}$ & 0.97642300 & 1. 36719800 & -3.79679700 \\
\hline 0 & 0.72158900 & 0.12064800 & -3.14239400 \\
\hline$r$ & -0.25302300 & 2. 27210200 & -4.01279700 \\
\hline
\end{tabular}




$\begin{array}{rrrr}\mathrm{H} & 0.05596800 & 2.98003700 & -4.80198300 \\ \mathrm{H} & -1.05677600 & 1.67009300 & -4.46846400 \\ \mathrm{C} & -0.81571700 & 3.10776900 & -2.84844000 \\ \mathrm{H} & -0.00800400 & 3.41522100 & -2.16222200 \\ \mathrm{H} & -1.23251100 & 4.04170400 & -3.25109300 \\ \mathrm{C} & -1.93400100 & 2.50080600 & -1.98587400 \\ \mathrm{O} & -2.87524600 & 3.23843000 & -1.66516300 \\ \mathrm{O} & -1.84645900 & 1.27586700 & -1.60803500 \\ \mathrm{H} & 1.30257900 & 1.07444100 & -4.81417100 \\ \mathrm{O} & -1.19791800 & -1.15513800 & -3.33476700 \\ \mathrm{H} & -0.16637000 & -0.48640600 & -3.61061900 \\ \mathrm{C} & -2.42462700 & -0.87563300 & -3.95684400 \\ \mathrm{H} & -3.22770500 & -1.50819100 & -3.53741700 \\ \mathrm{H} & -2.74092700 & 0.17766200 & -3.84922100 \\ \mathrm{H} & -2.35559100 & -1.10819800 & -5.03689700\end{array}$

\section{IntOR}

Ir

$\mathrm{P}$

0

$\mathrm{N}$

C

C

C

C

C

C

C

C

C

C

C

C

C

C

C

C

C

C

C

C

C

C

C
$-0.45408300$

0. 85205800

$-2.72702800$

$-1.85489300$

$-3.07355500$

$-3.34669500$

$-1.81451300$

$-0.82643100$

$-0.99009000$

$-0.00258000$

1. 16642900

1. 33091500

2. 47299700

2. 20774400

0. 66822500

0. 33887200

$-0.08433600$

$-0.07360600$

$-0.08494500$

$-0.40219400$

$-0.32519500$

0. 06127400

0. 37505600

0. 29593000

2. 62485700

3. 47904500

4. 82718400
$-0.41943500$

0. 14315400

$-2.82227700$

$-1.28619400$

$-0.63719100$

$-1.56671100$

$-2.50154200$

$-3.51306800$

$-4.17502300$

$-5.04468500$

$-5.24656900$

$-4.58202600$

$-4.69268600$

$-3.53341500$

$-3.26698900$

$-3.71425600$

$-4.25602900$

$-3.58180000$

$-2.11830500$

$-1.09982100$

0. 21615000

0. 47975000

$-0.52441800$

$-1.88227000$

$-0.38528800$

$-0.26860200$

$-0.64383100$
$-1.48985200$

0. 33880100

1. 08693100

$-0.27297300$

0. 30311800

1. 49283200

0. 16363000

$-0.26568300$

$-1.49174400$

$-1.95784200$

$-1.21626400$

0. 00046400

0. 98407600

1. 96532700

1. 90216600

0. 48338200

2. 85491000

4. 22168900

3. 86105600

4. 75715300

4. 32048700

3. 00647400

2. 06417000

2. 51417400

0. 20318500

1. 31392800

1. 23353400 


\begin{tabular}{|c|c|c|c|}
\hline $\mathrm{C}$ & 5. 28847000 & -1.14463700 & 0.00293500 \\
\hline $\mathrm{C}$ & 4. 46599000 & -1.28049500 & -1.12529300 \\
\hline $\mathrm{C}$ & 3. 12220800 & -0.88603700 & -1.00588700 \\
\hline $\mathrm{C}$ & 5. 04542300 & -1.84517900 & -2.43961300 \\
\hline $\mathrm{C}$ & 3. 96875600 & -1.99742600 & -3.52984300 \\
\hline $\mathrm{C}$ & 5. 66225800 & -3.23911200 & -2.17972200 \\
\hline $\mathrm{C}$ & 6.14231400 & -0.88932600 & -2.96339600 \\
\hline $\mathrm{C}$ & 5. 79467000 & -0.54634600 & 2. 43213500 \\
\hline $\mathrm{C}$ & 5. 11180800 & 0.02916500 & 3. 68648100 \\
\hline $\mathrm{C}$ & 6. 32640100 & -1.95688100 & 2. 77797800 \\
\hline $\mathrm{C}$ & 6.98351800 & 0.37188300 & 2. 06533300 \\
\hline $\mathrm{C}$ & 0.98640300 & 1. 98619500 & 0.59847300 \\
\hline $\mathrm{C}$ & -0.19639300 & 2. 72960500 & 0.79788400 \\
\hline $\mathrm{C}$ & -0.15679600 & 4. 10757900 & 1. 03783400 \\
\hline $\mathrm{C}$ & 1. 09941800 & 4. 74539200 & 0.97507600 \\
\hline $\mathrm{C}$ & 2. 28944700 & 4. 05443300 & 0.73037100 \\
\hline $\mathrm{C}$ & 2.21300300 & 2. 65681400 & 0.56626200 \\
\hline $\mathrm{C}$ & 3. 66227900 & 4. 75712300 & 0.66285400 \\
\hline $\mathrm{C}$ & 4. 31369500 & 4. 49051600 & -0.71376700 \\
\hline $\mathrm{C}$ & 3.54850200 & 6.28066600 & 0.85147000 \\
\hline $\mathrm{C}$ & 4. 57765200 & 4. 20081300 & 1. 77791700 \\
\hline $\mathrm{C}$ & -1.41250600 & 4. 93663200 & 1. 38417500 \\
\hline $\mathrm{C}$ & -2.64577700 & 4. 04499400 & 1. 61698800 \\
\hline $\mathrm{C}$ & -1.72768600 & 5. 90964400 & 0.22376000 \\
\hline $\mathrm{C}$ & -1.15008000 & 5. 74126100 & 2. 67933100 \\
\hline $\mathrm{H}$ & 0.40145000 & -1.72214900 & -1.48292900 \\
\hline $\mathrm{H}$ & -2.82349100 & 0.38260300 & 0.61603500 \\
\hline $\mathrm{H}$ & -2.84160600 & -1.23869300 & 2. 41482700 \\
\hline $\mathrm{H}$ & -4.40694700 & -1.75452400 & 1. 69272100 \\
\hline $\mathrm{H}$ & -1.87514000 & -3.97562800 & -2.09775700 \\
\hline $\mathrm{H}$ & -0.13880800 & -5.55091700 & -2.91625800 \\
\hline $\mathrm{H}$ & 1.94346200 & -5.92135800 & -1.58643100 \\
\hline $\mathrm{H}$ & 2. 44472600 & -5.67429200 & 1. 49089100 \\
\hline $\mathrm{H}$ & 3. 46046100 & -4.61759500 & 0.50161100 \\
\hline $\mathrm{H}$ & 2. 74084200 & -2.63847100 & 1. 62537000 \\
\hline $\mathrm{H}$ & 2.54471200 & -3.74638000 & 2. 99131300 \\
\hline $\mathrm{H}$ & -1.12225900 & -4.35771900 & 2. 50483900 \\
\hline $\mathrm{H}$ & 0.37121900 & -5.25720900 & 2. 84224100 \\
\hline $\mathrm{H}$ & -0.92601100 & -3.86897100 & 4. 85768200 \\
\hline $\mathrm{H}$ & 0.84201500 & -3.82326400 & 4. 79174800 \\
\hline $\mathrm{H}$ & -0.69613300 & -1.34139400 & 5. 78225000 \\
\hline $\mathrm{H}$ & -0.55679600 & 1.04760100 & 4. 99080000 \\
\hline $\mathrm{H}$ & 0.12702000 & 1. 52305400 & 2. 71423300 \\
\hline $\mathrm{H}$ & 3. 07376300 & 0.10972200 & 2. 25164700 \\
\hline $\mathrm{H}$ & 6. 33491800 & -1.44644200 & -0.07518900 \\
\hline
\end{tabular}

S162 


\begin{tabular}{|c|c|c|c|}
\hline H & 2. 44700200 & -0.95926900 & -1.85501600 \\
\hline $\mathrm{H}$ & 3. 49987900 & -1.03718500 & -3.79016700 \\
\hline H & 3. 16632800 & -2.68464400 & -3.22074400 \\
\hline $\mathrm{H}$ & 4. 42449100 & -2.40506000 & -4.44601400 \\
\hline $\mathrm{H}$ & 6. 48125400 & -3.20295000 & -1.44532100 \\
\hline H & 6.07395500 & -3.65583400 & -3.11353800 \\
\hline $\mathrm{H}$ & 4. 90155200 & -3.94128500 & -1.80245900 \\
\hline H & 5.72766300 & 0.10967300 & -3.17249000 \\
\hline $\mathrm{H}$ & 6.57866100 & -1.27811800 & -3.89826900 \\
\hline H & 6.96191400 & -0.76650400 & -2.23836300 \\
\hline H & 4. 72928100 & 1. 04788100 & 3.51839600 \\
\hline $\mathrm{H}$ & 5.83732100 & 0.08212700 & 4. 51344400 \\
\hline H & 4. 27257100 & -0.59839300 & 4. 02422200 \\
\hline H & 5.50152000 & -2.63544000 & 3. 04793100 \\
\hline H & 7. 01972500 & -1.90695700 & 3. 63367800 \\
\hline $\mathrm{H}$ & 6.87104600 & -2.40998000 & 1.93558700 \\
\hline H & 6. 63798300 & 1. 38906400 & 1. 82215000 \\
\hline H & 7. 54576100 & -0.00707600 & 1. 19839800 \\
\hline H & 7. 68657700 & 0.44548500 & 2. 91111400 \\
\hline H & -1.15350800 & 2. 21257900 & 0.77545300 \\
\hline $\mathrm{H}$ & 1. 13522800 & 5. 82246500 & 1. 12956800 \\
\hline H & 3. 12790200 & 2. 09002100 & 0.40330500 \\
\hline $\mathrm{H}$ & 4. 46540800 & 3. 41611300 & -0.89744200 \\
\hline $\mathrm{H}$ & 5. 29924400 & 4. 98097800 & -0.77361400 \\
\hline $\mathrm{H}$ & 3. 68783200 & 4. 88514900 & -1.52975300 \\
\hline $\mathrm{H}$ & 4.54755500 & 6. 73985100 & 0.78670700 \\
\hline $\mathrm{H}$ & 3. 12883000 & 6.54458300 & 1. 83470300 \\
\hline $\mathrm{H}$ & 2. 92032100 & 6.74531000 & 0.07557700 \\
\hline $\mathrm{H}$ & 4. 74093300 & 3. 11783000 & 1. 66979900 \\
\hline $\mathrm{H}$ & 4. 14003300 & 4. 37720800 & 2. 77350700 \\
\hline $\mathrm{H}$ & 5.56463500 & 4. 69146100 & 1. 74834100 \\
\hline $\mathrm{H}$ & -2.47914200 & 3.32507400 & 2. 43547400 \\
\hline $\mathrm{H}$ & -2.92451600 & 3. 50179300 & 0.70302800 \\
\hline $\mathrm{H}$ & -3.50481100 & 4. 67317400 & 1. 90195300 \\
\hline $\mathrm{H}$ & -0.86676800 & 6.55578500 & -0.01259300 \\
\hline $\mathrm{H}$ & -2.57062400 & 6.56525000 & 0.49969500 \\
\hline $\mathrm{H}$ & -2.01658100 & 5.34951500 & -0.67810200 \\
\hline $\mathrm{H}$ & -0.33246600 & 6. 46988100 & 2. 56767100 \\
\hline $\mathrm{H}$ & -0.89432700 & 5. 07439700 & 3. 51911100 \\
\hline $\mathrm{H}$ & -2.05417300 & 6.30565000 & 2. 95955400 \\
\hline $\mathrm{C}$ & -4.20694500 & -0.57027600 & -0.74446600 \\
\hline $\mathrm{H}$ & -4.42442500 & -1.58782000 & -1.10236400 \\
\hline $\mathrm{H}$ & -3.80022800 & -0.00213400 & -1.58805300 \\
\hline $\mathrm{C}$ & -5.45475700 & 0.12817300 & -0.23881000 \\
\hline$C$ & -6.59110700 & -0.58888200 & 0.27591700 \\
\hline
\end{tabular}




$\begin{array}{lrrr}\mathrm{C} & -5.48766500 & 1.51584600 & -0.27615600 \\ \mathrm{C} & -6.67310100 & -2.01393300 & 0.31690400 \\ \mathrm{C} & -7.72277000 & 0.15638500 & 0.76590900 \\ \mathrm{C} & -6.60446900 & 2.23889300 & 0.20705000 \\ \mathrm{H} & -4.64045300 & 2.06652100 & -0.70284300 \\ \mathrm{C} & -7.78423500 & -2.66195700 & 0.81768100 \\ \mathrm{H} & -5.84394200 & -2.61360500 & -0.06135500 \\ \mathrm{C} & -8.85163500 & -0.54433800 & 1.27811700 \\ \mathrm{C} & -7.69633500 & 1.57704500 & 0.72336900 \\ \mathrm{H} & -6.59088100 & 3.33079400 & 0.15979000 \\ \mathrm{C} & -8.88716400 & -1.92152400 & 1.30845600 \\ \mathrm{H} & -7.81502300 & -3.75463100 & 0.83073400 \\ \mathrm{H} & -9.70046400 & 0.03808800 & 1.64775100 \\ \mathrm{H} & -8.56147200 & 2.13102100 & 1.09861300 \\ \mathrm{H} & -9.76255800 & -2.44330100 & 1.70345300 \\ \mathrm{C} & 2.12901000 & 2.20964600 & -3.14504500 \\ \mathrm{H} & 2.34349700 & 3.15241700 & -3.67240200 \\ \mathrm{H} & 3.04019300 & 1.59329500 & -3.15728200 \\ \mathrm{H} & 1.89571100 & 2.44967700 & -2.09924500 \\ \mathrm{C} & 0.98309100 & 1.47815500 & -3.82395900 \\ \mathrm{O} & 0.77587300 & 0.20982400 & -3.16415100 \\ \mathrm{C} & -0.28833000 & 2.32105600 & -4.02662500 \\ \mathrm{H} & -0.02489900 & 3.02711800 & -4.83373100 \\ \mathrm{H} & -1.07234700 & 1.67440400 & -4.45417800 \\ \mathrm{C} & -0.86006700 & 3.15026200 & -2.86259500 \\ \mathrm{H} & -0.05491100 & 3.48074600 & -2.18430600 \\ \mathrm{H} & -1.30158500 & 4.07176700 & -3.26720900 \\ \mathrm{C} & -1.95526600 & 2.52348900 & -1.98312400 \\ \mathrm{O} & -2.89322700 & 3.25387800 & -1.63596800 \\ \mathrm{O} & -1.84732800 & 1.29833400 & -1.61932900 \\ \mathrm{H} & 1.32132000 & 1.19504200 & -4.83819000 \\ \mathrm{H} & -1.18917000 & -1.12940400 & -3.34040600 \\ \mathrm{H} & 0.05405100 & -0.34231100 & -3.66975800 \\ \mathrm{C} & -2.45271800 & -0.93146300 & -3.89680500 \\ \mathrm{H} & -1.58195000 & -3.43023000 \\ & -2.8193900 & 0.11081300 & -3.81762500 \\ \mathrm{H} & -1.19519400 & -4.97357200\end{array}$

\section{IntPR}

$\begin{array}{rrrr}\text { Ir } & -0.51406500 & -0.25147500 & -1.29439800 \\ \mathrm{P} & 1.14525000 & 0.07996900 & 0.38042000 \\ 0 & -2.25211400 & -3.41142400 & 0.91449200 \\ \mathrm{~N} & -1.66389800 & -1.51905700 & -0.10143600 \\ \mathrm{C} & -2.94857100 & -1.18306100 & 0.57695600\end{array}$




\begin{tabular}{|c|c|c|c|}
\hline $\mathrm{C}$ & -3.13040400 & -2.40547800 & 1. 49371800 \\
\hline $\mathrm{C}$ & -1.41576100 & -2.76717800 & 0.09840100 \\
\hline $\mathrm{C}$ & -0.30394400 & -3.54476300 & -0.49293100 \\
\hline $\mathrm{C}$ & -0.44720600 & -4.07112700 & -1.78684400 \\
\hline $\mathrm{C}$ & 0.59105000 & -4.80331600 & -2.36467100 \\
\hline $\mathrm{C}$ & 1. 78316400 & -5.01172500 & -1.66101700 \\
\hline $\mathrm{C}$ & 1.92831000 & -4.47427600 & -0.38139200 \\
\hline $\mathrm{C}$ & 3. 08751300 & -4.63742000 & 0.57343600 \\
\hline $\mathrm{C}$ & 2. 77635000 & -3.62094700 & 1. 68955600 \\
\hline $\mathrm{C}$ & 1. 22470600 & -3.41141600 & 1. 66339100 \\
\hline $\mathrm{C}$ & 0.89284500 & -3.72947800 & 0.20918900 \\
\hline $\mathrm{C}$ & 0.53301500 & -4.50680900 & 2. 54060400 \\
\hline $\mathrm{C}$ & 0.55746300 & -3.95434500 & 3. 96326000 \\
\hline $\mathrm{C}$ & 0.52173900 & -2.46252500 & 3. 74216600 \\
\hline $\mathrm{C}$ & 0.26161000 & -1.52988100 & 4. 74508000 \\
\hline $\mathrm{C}$ & 0.33302100 & -0.17857600 & 4. 43283200 \\
\hline $\mathrm{C}$ & 0.63621200 & 0.20951700 & 3. 12768800 \\
\hline $\mathrm{C}$ & 0.87495600 & -0.70449200 & 2. 08139800 \\
\hline $\mathrm{C}$ & 0.83173700 & -2.09904000 & 2. 40744200 \\
\hline $\mathrm{C}$ & 2. 86019000 & -0.49102700 & -0.05239700 \\
\hline $\mathrm{C}$ & 3.87738000 & -0.35755300 & 0.90891000 \\
\hline $\mathrm{C}$ & 5. 20377000 & -0.69993700 & 0.61562000 \\
\hline $\mathrm{C}$ & 5. 48039500 & -1.16799300 & -0.68205600 \\
\hline $\mathrm{C}$ & 4. 49652100 & -1.30689500 & -1.67154300 \\
\hline $\mathrm{C}$ & 3. 17555900 & -0.96619700 & -1.32971800 \\
\hline $\mathrm{C}$ & 4. 88438300 & -1.80807700 & -3.07867800 \\
\hline $\mathrm{C}$ & 3. 66761600 & -1.90260700 & -4.01734800 \\
\hline $\mathrm{C}$ & 5.52079200 & -3.21319800 & -2.97140100 \\
\hline $\mathrm{C}$ & 5. 90529500 & -0.83059500 & -3.70642000 \\
\hline $\mathrm{C}$ & 6.34131400 & -0.59631900 & 1. 65365000 \\
\hline $\mathrm{C}$ & 5. 85258100 & -0.02122500 & 2. 99543700 \\
\hline $\mathrm{C}$ & 6.92154100 & -2.00646000 & 1. 91281000 \\
\hline $\mathrm{C}$ & 7. 45917500 & 0.32471700 & 1. 11343400 \\
\hline $\mathrm{C}$ & 1. 45725400 & 1. 91231800 & 0.61558000 \\
\hline $\mathrm{C}$ & 0.51879500 & 2. 75242400 & 1. 25200800 \\
\hline $\mathrm{C}$ & 0.75644400 & 4. 12758400 & 1. 38542000 \\
\hline $\mathrm{C}$ & 1. 89443400 & 4. 66615100 & 0.75406600 \\
\hline $\mathrm{C}$ & 2. 80149900 & 3. 88070200 & 0.03849900 \\
\hline $\mathrm{C}$ & 2.58035300 & 2. 49112100 & 0.00929700 \\
\hline $\mathrm{C}$ & 4. 02029200 & 4. 47307900 & -0.70020200 \\
\hline $\mathrm{C}$ & 3.92173200 & 4. 12639400 & -2.20425900 \\
\hline $\mathrm{C}$ & 4. 09673100 & 6. 00480100 & -0.56630600 \\
\hline $\mathrm{C}$ & 5. 32227000 & 3. 87349600 & -0.12152900 \\
\hline $\mathrm{C}$ & -0.15488700 & 5. 06125200 & 2. 21274900 \\
\hline $\mathrm{C}$ & -1.28379500 & 4. 29643800 & 2. 92729100 \\
\hline
\end{tabular}

S165 


\begin{tabular}{|c|c|c|c|}
\hline $\mathrm{C}$ & -0.77885900 & 6.14086100 & 1. 29891600 \\
\hline $\mathrm{C}$ & 0.70165600 & 5. 75738700 & 3. 29900200 \\
\hline H & 0.33875700 & -1.49127500 & -1.70486300 \\
\hline $\mathrm{H}$ & -2.79983200 & -0.25104700 & 1. 13858500 \\
\hline $\mathrm{H}$ & -2.78680100 & -2.22600000 & 2. 52413900 \\
\hline $\mathrm{H}$ & -4.14978200 & -2.81054200 & 1. 50768500 \\
\hline $\mathrm{H}$ & -1.36262100 & -3.87011100 & -2.34632300 \\
\hline H & 0.47013400 & -5.20786700 & -3.37240500 \\
\hline $\mathrm{H}$ & 2.59134600 & -5.59705600 & -2.10866500 \\
\hline H & 3. 11450400 & -5.67320900 & 0.95774500 \\
\hline $\mathrm{H}$ & 4. 06361100 & -4.45416400 & 0.09706100 \\
\hline $\mathrm{H}$ & 3. 27210500 & -2.67040100 & 1. 46542500 \\
\hline $\mathrm{H}$ & 3. 12488600 & -3.94625200 & 2. 68137900 \\
\hline $\mathrm{H}$ & -0.50749900 & -4.62690700 & 2. 20657900 \\
\hline $\mathrm{H}$ & 1. 03005400 & -5.48217000 & 2. 43003600 \\
\hline $\mathrm{H}$ & -0.28187400 & -4.31045700 & 4. 58191100 \\
\hline $\mathrm{H}$ & 1. 48266600 & -4.23560100 & 4. 49831400 \\
\hline $\mathrm{H}$ & 0.02115500 & -1.86483000 & 5. 75785200 \\
\hline $\mathrm{H}$ & 0.15140300 & 0.58561100 & 5. 19243100 \\
\hline $\mathrm{H}$ & 0.68849300 & 1. 27440100 & 2. 92728600 \\
\hline $\mathrm{H}$ & 3. 61839100 & 0.02221800 & 1. 89666300 \\
\hline $\mathrm{H}$ & 6.50987100 & -1.43352100 & -0.93043500 \\
\hline $\mathrm{H}$ & 2.37680700 & -1.07544800 & -2.05935900 \\
\hline $\mathrm{H}$ & 3. 19124600 & -0.92223500 & -4.17395000 \\
\hline $\mathrm{H}$ & 2. 90276400 & -2.59517900 & -3.63434600 \\
\hline $\mathrm{H}$ & 3. 98716200 & -2.27215100 & -5.00440700 \\
\hline $\mathrm{H}$ & 6. 42678900 & -3.21154200 & -2.34585300 \\
\hline $\mathrm{H}$ & 5. 80835300 & -3.58144500 & -3.96975000 \\
\hline $\mathrm{H}$ & 4. 81058800 & -3.93388700 & -2.53626300 \\
\hline $\mathrm{H}$ & 5. 47807800 & 0.18049400 & -3.80068800 \\
\hline $\mathrm{H}$ & 6. 19404200 & -1.17190700 & -4.71405500 \\
\hline $\mathrm{H}$ & 6.82464600 & -0.75129100 & -3.10604400 \\
\hline $\mathrm{H}$ & 5. 43916500 & 0.99283200 & 2. 88156500 \\
\hline $\mathrm{H}$ & 6.69547700 & 0.04169700 & 3. 70138400 \\
\hline $\mathrm{H}$ & 5. 08077600 & -0.65427700 & 3. 46008800 \\
\hline $\mathrm{H}$ & 6. 14807800 & -2.68687600 & 2. 30317300 \\
\hline $\mathrm{H}$ & 7. 73590300 & -1.95745100 & 2. 65429700 \\
\hline $\mathrm{H}$ & 7. 33287900 & -2.45635000 & 0.99606000 \\
\hline $\mathrm{H}$ & 7. 07728700 & 1. 33959600 & 0.92165300 \\
\hline $\mathrm{H}$ & 7. 89158900 & -0.05484000 & 0.17512100 \\
\hline $\mathrm{H}$ & 8.27842500 & 0.40328500 & 1. 84673000 \\
\hline $\mathrm{H}$ & -0.41230300 & 2. 32686100 & 1. 62267700 \\
\hline $\mathrm{H}$ & 2. 06383000 & 5. 73935100 & 0.82534500 \\
\hline $\mathrm{H}$ & 3. 29749200 & 1. 85908100 & -0.51129700 \\
\hline $\mathrm{H}$ & 3. 91230700 & 3. 03888500 & -2.37401000 \\
\hline
\end{tabular}




\begin{tabular}{|c|c|c|c|}
\hline H & 4. 78274600 & 4. 54207500 & -2.75357600 \\
\hline $\mathrm{H}$ & 3. 00238900 & 4. 54283000 & -2.64578200 \\
\hline $\mathrm{H}$ & 4. 97294800 & 6.38291400 & -1.11636200 \\
\hline $\mathrm{H}$ & 4. 20427500 & 6.32201500 & 0.48270100 \\
\hline H & 3. 20574500 & 6.49935600 & -0.98338600 \\
\hline $\mathrm{H}$ & 5.35388800 & 2. 77924200 & -0.23302000 \\
\hline $\mathrm{H}$ & 5. 42102500 & 4. 10621600 & 0.95092000 \\
\hline $\mathrm{H}$ & 6. 20219500 & 4. 28699600 & -0.64191000 \\
\hline $\mathrm{H}$ & -0.88011000 & 3. 56985300 & 3. 65162800 \\
\hline H & -1.93471900 & 3. 74180000 & 2. 23735200 \\
\hline $\mathrm{H}$ & -1.90804900 & 5. 00779600 & 3. 49154700 \\
\hline H & -0.00683300 & 6.73916300 & 0.78929900 \\
\hline H & -1.39057600 & 6.83575800 & 1. 89817100 \\
\hline $\mathrm{H}$ & -1.43205700 & 5. 71132000 & 0.52565200 \\
\hline H & 1. 49509100 & 6.38727000 & 2. 86839100 \\
\hline $\mathrm{H}$ & 1. 18124300 & 5. 01849100 & 3. 96128700 \\
\hline $\mathrm{H}$ & 0.06566500 & 6. 40762700 & 3. 92197500 \\
\hline $\mathrm{C}$ & -4.08974400 & -0.99153200 & -0.44488700 \\
\hline $\mathrm{H}$ & -4.10705900 & -1.85603900 & -1.11913000 \\
\hline $\mathrm{H}$ & -3.82086000 & -0.14087900 & -1.08578800 \\
\hline $\mathrm{C}$ & -5.43174100 & -0.76261000 & 0.22864000 \\
\hline $\mathrm{C}$ & -6.54329500 & -1.66099500 & 0.05170200 \\
\hline $\mathrm{C}$ & -5.58815200 & 0.34347300 & 1. 05400000 \\
\hline $\mathrm{C}$ & -6.49220200 & -2.82821600 & -0.76893700 \\
\hline $\mathrm{C}$ & -7.78374900 & -1.38170400 & 0.73149500 \\
\hline $\mathrm{C}$ & -6.80856900 & 0.61037800 & 1. 71806500 \\
\hline $\mathrm{H}$ & -4.74483400 & 1. 02543300 & 1. 19839600 \\
\hline $\mathrm{C}$ & -7.58137800 & -3.66348600 & -0.90967600 \\
\hline $\mathrm{H}$ & -5.57461500 & -3.06977500 & -1.30555100 \\
\hline $\mathrm{C}$ & -8.88862300 & -2.26393300 & 0.56189300 \\
\hline $\mathrm{C}$ & -7.88606400 & -0.23240400 & 1. 56118300 \\
\hline $\mathrm{H}$ & -6.88691900 & 1. 49416000 & 2. 35663100 \\
\hline $\mathrm{C}$ & -8.79585800 & -3.38152200 & -0.23821500 \\
\hline $\mathrm{H}$ & -7.50671200 & -4.54789500 & -1.54781500 \\
\hline $\mathrm{H}$ & -9.82157900 & -2.03418400 & 1. 08451500 \\
\hline $\mathrm{H}$ & -8.83297100 & -0.03233200 & 2. 07037700 \\
\hline $\mathrm{H}$ & -9.65368500 & -4.04823400 & -0.35798000 \\
\hline $\mathrm{C}$ & -5.17945400 & 5. 11778900 & -2.47981000 \\
\hline $\mathrm{H}$ & -5.40220600 & 4. 39499000 & -3.28130800 \\
\hline $\mathrm{H}$ & -5.64295100 & 6. 07869900 & -2.76401300 \\
\hline $\mathrm{H}$ & -5.66435400 & 4. 77136800 & -1.55420700 \\
\hline $\mathrm{C}$ & -3.67098200 & 5. 28099000 & -2.28717600 \\
\hline 0 & -3.38634400 & 6.20961100 & -1.24302300 \\
\hline $\mathrm{C}$ & -2.93279700 & 3. 98259900 & -1.95072700 \\
\hline $\mathrm{H}$ & -3.05335800 & 3. 27491000 & -2.78588200 \\
\hline
\end{tabular}

S167 


$\begin{array}{rrrr}\mathrm{H} & -1.85645600 & 4.21502000 & -1.90296400 \\ \mathrm{C} & -3.36162400 & 3.32534700 & -0.63796000 \\ \mathrm{H} & -3.38010500 & 4.06427200 & 0.17603600 \\ \mathrm{H} & -4.39386400 & 2.93760900 & -0.71381000 \\ \mathrm{C} & -2.50332900 & 2.13744100 & -0.18204000 \\ \mathrm{O} & -2.53267000 & 1.81788200 & 1.02238200 \\ \mathrm{O} & -1.85203000 & 1.54179700 & -1.10170200 \\ \mathrm{H} & -3.24588700 & 5.65820900 & -3.24154700 \\ \mathrm{O} & -1.88275100 & -0.80039200 & -2.72592800 \\ \mathrm{H} & -3.86387900 & 7.02904900 & -1.42422600 \\ \mathrm{C} & -2.05683600 & -0.01267200 & -3.85499500 \\ \mathrm{H} & -2.32046100 & 1.04024500 & -3.62317900 \\ \mathrm{H} & -1.16286500 & 0.01552300 & -4.52500900 \\ \mathrm{H} & -2.88663900 & -0.42773600 & -4.46393800 \\ \mathrm{H} & 0.38279600 & 0.33422800 & -2.59052300 \\ \mathrm{H} & 0.31001500 & 1.03277800 & -2.02971200\end{array}$

\section{TSPR}

Ir

$\mathrm{P}$

0

$\mathrm{N}$

C

C

C

C

C

C

C

C

C

C

C

C

C

C

C

C

C

C

C

C

C

C

$\begin{array}{rrr}-0.43299400 & -0.30711900 & -1.38534600 \\ 1.01915400 & -0.14238800 & 0.41829200 \\ -2.78573000 & -3.11785300 & 0.74965800 \\ -1.88254100 & -1.34693200 & -0.26516100 \\ -3.09063600 & -0.80996800 & 0.42649900 \\ -3.42066100 & -1.96679800 & 1.37879900 \\ -1.86386700 & -2.62493700 & -0.08271900 \\ -0.91099900 & -3.56130500 & -0.71953500 \\ -1.15769500 & -4.00904900 & -2.02624000 \\ -0.22522400 & -4.81394800 & -2.68298100 \\ 0.96915400 & -5.16885400 & -2.04716600 \\ 1.21523600 & -4.71953800 & -0.74832000 \\ 2.39495300 & -5.03643000 & 0.14093000 \\ 2.22499400 & -4.05607100 & 1.31707600 \\ 0.69649200 & -3.72401800 & 1.37933800 \\ 0.28206800 & -3.91294300 & -0.07633300 \\ -0.06838900 & -4.82242100 & 2.19029200 \\ 0.04951900 & -4.40482500 & 3.65195700 \\ 0.16639700 & -2.90463400 & 3.56065900 \\ 0.06759500 & -2.05139600 & 4.65835700 \\ 0.31111900 & -0.69751500 & 4.47123000 \\ 0.60917400 & -0.22568200 & 3.19258700 \\ 0.66518400 & -1.04984000 & 2.04877300 \\ 0.46127600 & -2.45313800 & 2.24856700 \\ 2.75324100 & -0.70295700 & 0.06057400 \\ 3.70872800 & -0.62998300 & 1.08848900\end{array}$

\section{S168}




\begin{tabular}{|c|c|c|c|}
\hline $\mathrm{C}$ & 5. 04159800 & -0.99809200 & 0.86636200 \\
\hline $\mathrm{C}$ & 5. 38641200 & -1.43990400 & -0.42394100 \\
\hline $\mathrm{C}$ & 4. 46244200 & -1.52653500 & -1.47531000 \\
\hline $\mathrm{C}$ & 3. 13352700 & -1.15258700 & -1.20851200 \\
\hline $\mathrm{C}$ & 4. 92145500 & -2.00867600 & -2.86748200 \\
\hline $\mathrm{C}$ & 3. 76213700 & -2.04506800 & -3.87973100 \\
\hline $\mathrm{C}$ & 5. 50963600 & -3.43428300 & -2.75518600 \\
\hline $\mathrm{C}$ & 6.00732900 & -1.04918400 & -3.40810600 \\
\hline $\mathrm{C}$ & 6.11816200 & -0.93529100 & 1.96984400 \\
\hline $\mathrm{C}$ & 5.55185900 & -0.42233800 & 3. 30643900 \\
\hline $\mathrm{C}$ & 6. 69824400 & -2.34981900 & 2. 20149800 \\
\hline $\mathrm{C}$ & 7. 25201100 & 0.01948100 & 1. 52921900 \\
\hline $\mathrm{C}$ & 1. 28754500 & 1. 65633900 & 0.83998200 \\
\hline $\mathrm{C}$ & 0.35692000 & 2. 39941200 & 1. 59261500 \\
\hline $\mathrm{C}$ & 0.58351000 & 3. 74832000 & 1. 89355900 \\
\hline $\mathrm{C}$ & 1. 70075200 & 4. 37352500 & 1. 30928600 \\
\hline C & 2.58634000 & 3. 69824200 & 0.46353800 \\
\hline $\mathrm{C}$ & 2.38341300 & 2. 31868400 & 0.27396500 \\
\hline $\mathrm{C}$ & 3.72529100 & 4. 41480200 & -0.28940000 \\
\hline $\mathrm{C}$ & 3.39483700 & 4. 38851800 & -1.80189200 \\
\hline $\mathrm{C}$ & 3. 87382600 & 5.88512800 & 0.14289400 \\
\hline $\mathrm{C}$ & 5. 07304000 & 3. 70277100 & -0.03712100 \\
\hline $\mathrm{C}$ & -0.30720200 & 4. 53830100 & 2. 87891700 \\
\hline $\mathrm{C}$ & -1.54336200 & 3. 73220500 & 3. 31864600 \\
\hline $\mathrm{C}$ & -0.79718500 & 5. 85956700 & 2. 24503900 \\
\hline C & 0.53892600 & 4. 86820800 & 4. 13257300 \\
\hline $\mathrm{H}$ & 0.25057400 & -1.67484200 & -1.67735000 \\
\hline $\mathrm{H}$ & -2.82377900 & 0.12273300 & 0.94001400 \\
\hline $\mathrm{H}$ & -2.95911600 & -1.84498200 & 2. 37139900 \\
\hline $\mathrm{H}$ & -4.49005700 & -2.18115200 & 1. 48743700 \\
\hline $\mathrm{H}$ & -2.06315100 & -3.68577500 & -2.54243900 \\
\hline $\mathrm{H}$ & -0.42551200 & -5.14884600 & -3.70332700 \\
\hline $\mathrm{H}$ & 1. 70177000 & -5.79661300 & -2.56227600 \\
\hline $\mathrm{H}$ & 2. 34629100 & -6.08897600 & 0.47398400 \\
\hline $\mathrm{H}$ & 3. 36200400 & -4.91465000 & -0.37227800 \\
\hline $\mathrm{H}$ & 2. 78282900 & -3.13396000 & 1. 10995300 \\
\hline $\mathrm{H}$ & 2. 59520300 & -4.45444100 & 2. 27385300 \\
\hline $\mathrm{H}$ & -1.12545900 & -4.80749800 & 1. 88619900 \\
\hline $\mathrm{H}$ & 0.32220000 & -5.82856800 & 1. 97742000 \\
\hline $\mathrm{H}$ & -0.80669100 & -4.72653300 & 4. 26642600 \\
\hline $\mathrm{H}$ & 0.95385500 & -4.82378300 & 4. 12929200 \\
\hline $\mathrm{H}$ & -0.16962900 & -2.45361000 & 5. 64712600 \\
\hline $\mathrm{H}$ & 0.27679600 & 0.00295300 & 5. 30932300 \\
\hline $\mathrm{H}$ & 0.81636900 & 0.83453200 & 3. 09473000 \\
\hline $\mathrm{H}$ & 3. 39770000 & -0.27758000 & 2. 07086200 \\
\hline
\end{tabular}




\begin{tabular}{|c|c|c|c|}
\hline H & 6.42238500 & -1.72629400 & -0.61571600 \\
\hline $\mathrm{H}$ & 2.37835000 & -1.21478800 & -1.98810100 \\
\hline H & 3. 32324000 & -1.04817300 & -4.03984700 \\
\hline $\mathrm{H}$ & 2.95516800 & -2.72223800 & -3.56047500 \\
\hline H & 4. 13034600 & -2.40177300 & -4.85449200 \\
\hline H & 6.37515500 & -3.47430900 & -2.07589300 \\
\hline H & 5.84664300 & -3.78941900 & -3.74278800 \\
\hline H & 4. 75480700 & -4.14405700 & -2.38071600 \\
\hline H & 5. 61703900 & -0.02327900 & -3.50089200 \\
\hline H & 6.34608000 & -1.37526300 & -4.40519400 \\
\hline H & 6.89065200 & -1.01381800 & -2.75209900 \\
\hline H & 5. 14648400 & 0.59738100 & 3. 21647200 \\
\hline H & 6.35227800 & -0.39429000 & 4. 06241600 \\
\hline H & 4. 75390800 & -1.07429000 & 3. 69456500 \\
\hline H & 5.91156000 & -3.05215600 & 2. 52034200 \\
\hline H & 7. 47061300 & -2.32483000 & 2. 98779200 \\
\hline H & 7. 16411100 & -2.75861200 & 1. 29175800 \\
\hline H & 6.86756400 & 1. 03810000 & 1. 36190800 \\
\hline H & 7. 73324100 & -0.31471700 & 0.59749200 \\
\hline H & 8.03282000 & 0.07301500 & 2. 30560400 \\
\hline H & -0.55820100 & 1. 92618700 & 1. 93766000 \\
\hline H & 1. 86618500 & 5.43000000 & 1. 51395100 \\
\hline $\mathrm{H}$ & 3. 08467200 & 1. 76012700 & -0.34526100 \\
\hline $\mathrm{H}$ & 3. 33240800 & 3.35714200 & -2.18271000 \\
\hline H & 4. 17850800 & 4. 91095800 & -2.37654300 \\
\hline $\mathrm{H}$ & 2.42774400 & 4. 87705600 & -2.00122100 \\
\hline H & 4. 70186400 & 6.35383100 & -0.41213400 \\
\hline H & 4. 09951800 & 5. 97853200 & 1. 21727600 \\
\hline $\mathrm{H}$ & 2.96407100 & 6.46733100 & -0.06904500 \\
\hline $\mathrm{H}$ & 5. 05875200 & 2. 65419100 & -0.37010400 \\
\hline $\mathrm{H}$ & 5.33206400 & 3. 71265700 & 1. 03424900 \\
\hline $\mathrm{H}$ & 5. 88274100 & 4. 21114400 & -0.58619900 \\
\hline $\mathrm{H}$ & -1.26141600 & 2. 82640700 & 3. 87954400 \\
\hline $\mathrm{H}$ & -2.15413200 & 3. 42298900 & 2. 45707500 \\
\hline $\mathrm{H}$ & -2.16639200 & 4. 34671600 & 3. 98830900 \\
\hline $\mathrm{H}$ & 0.03430400 & 6.50141200 & 1. 91650700 \\
\hline $\mathrm{H}$ & -1.38776800 & 6.43318500 & 2. 97808400 \\
\hline $\mathrm{H}$ & -1.43681900 & 5.66643400 & 1. 37193100 \\
\hline $\mathrm{H}$ & 1. 41350800 & 5. 49087200 & 3. 88640600 \\
\hline $\mathrm{H}$ & 0.90821600 & 3.94878500 & 4. 61502500 \\
\hline $\mathrm{H}$ & -0.06789800 & 5. 41827800 & 4. 87106400 \\
\hline $\mathrm{C}$ & -4.19298700 & -0.48472900 & -0.60796000 \\
\hline $\mathrm{H}$ & -4.30128700 & -1.33323200 & -1.29778100 \\
\hline $\mathrm{H}$ & -3.82103700 & 0.35751200 & -1.20593000 \\
\hline 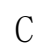 & -5.52130200 & -0.10534600 & 0.01988900 \\
\hline
\end{tabular}




$\begin{array}{lrrr}\mathrm{C} & -6.64242200 & -1.00737700 & 0.04821300 \\ \mathrm{C} & -5.65096600 & 1.15231600 & 0.59234100 \\ \mathrm{C} & -6.60773100 & -2.32668500 & -0.49643600 \\ \mathrm{C} & -7.87427200 & -0.57078700 & 0.65551100 \\ \mathrm{C} & -6.86147400 & 1.56994600 & 1.19423300 \\ \mathrm{H} & -4.79006200 & 1.82762000 & 0.57608300 \\ \mathrm{C} & -7.70871400 & -3.15750000 & -0.45200400 \\ \mathrm{H} & -5.69150400 & -2.69649700 & -0.95784000 \\ \mathrm{C} & -8.99227600 & -1.45234200 & 0.67970700 \\ \mathrm{C} & -7.95270200 & 0.73011000 & 1.22286300 \\ \mathrm{H} & -6.92359900 & 2.56966200 & 1.63213000 \\ \mathrm{C} & -8.91797100 & -2.71766100 & 0.13936200 \\ \mathrm{H} & -7.64734100 & -4.16201300 & -0.87877500 \\ \mathrm{H} & -9.92016700 & -1.10225900 & 1.14095700 \\ \mathrm{H} & -8.89298100 & 1.04889500 & 1.68137300 \\ \mathrm{H} & -9.78561200 & -3.38182700 & 0.16600700 \\ \mathrm{C} & -2.34300900 & 6.75493200 & -2.55014800 \\ \mathrm{H} & -3.25013600 & 6.51910800 & -3.12985100 \\ \mathrm{H} & -2.02344900 & 7.77197600 & -2.83769800 \\ \mathrm{H} & -2.60884600 & 6.77393400 & -1.48201800 \\ \mathrm{C} & -1.23468100 & 5.73786000 & -2.82555400 \\ \mathrm{O} & -0.06462600 & 6.04419500 & -2.06387500 \\ \mathrm{C} & -1.61373600 & 4.28684000 & -2.51478000 \\ \mathrm{H} & -2.44490600 & 3.99340800 & -3.17918400 \\ \mathrm{H} & -0.75975700 & 3.64969300 & -2.78663800 \\ \mathrm{C} & -1.96861000 & 4.01154500 & -1.05317700 \\ \mathrm{H} & -1.20887600 & 4.49422100 & -0.41669200 \\ \mathrm{H} & -2.93839600 & 4.44608200 & -0.76450800 \\ \mathrm{C} & -1.97311600 & 2.53517400 & -0.62512500 \\ \mathrm{H} & -2.56646700 & 2.22750200 & 0.42610600 \\ \mathrm{H} & -1.31362700 & 1.74560600 & -1.37628500 \\ \mathrm{H} & -0.98891800 & 5.78510000 & -3.90716200 \\ \mathrm{H} & -1.27487200 & -0.54170600 & -3.34655600 \\ \mathrm{H} & -.17386100 & 6.96436700 & -2.23423100 \\ \mathrm{H} & -26568300 & 0.29852600 & -3.87000100 \\ \mathrm{H} & -2.160800 & 0.14364600 & -3.38690800 \\ \mathrm{H} & -3854500 & 1.37404800 & -3.77712200 \\ \mathrm{H} & 0.06844100 & -4.94754900 \\ \mathrm{H} & 0.17239800 & -2.95920100 \\ \mathrm{H} & 0.49566000 & -2.36558400\end{array}$




\begin{tabular}{|c|c|c|c|}
\hline Ir & -1.07569500 & 0.76357200 & -0.53644500 \\
\hline Ir & 1. 03858000 & -0.84656400 & 0.06564200 \\
\hline $\mathrm{P}$ & -3.34557600 & 0.39698600 & -0.69447100 \\
\hline $\mathrm{P}$ & 3. 37015000 & -0.78793800 & 0.17759900 \\
\hline 0 & -2.02103500 & 4. 52581100 & -2.35642700 \\
\hline 0 & 1. 67057100 & -4.85577200 & 1. 58463300 \\
\hline 0 & 0.87258700 & 0.07469700 & 2. 10931200 \\
\hline 0 & -0.76650600 & 1. 52201800 & 1. 60008900 \\
\hline $\mathrm{N}$ & -1.38579500 & 2. 82616900 & -1.05430700 \\
\hline $\mathrm{N}$ & 1. 07801100 & -2.78251700 & 1. 00444300 \\
\hline $\mathrm{C}$ & -1.67044800 & 3. 94682800 & -0.11225000 \\
\hline $\mathrm{C}$ & -2.42527400 & 4. 93161200 & -1.02066800 \\
\hline $\mathrm{C}$ & -1.56081100 & 3. 27623600 & -2.25242500 \\
\hline $\mathrm{C}$ & -1.35250700 & 2. 50928600 & -3.49745700 \\
\hline $\mathrm{C}$ & -0.06162000 & 2. 30417400 & -4.00597300 \\
\hline $\mathrm{C}$ & 0. 12378200 & 1. 54546900 & -5.16305900 \\
\hline $\mathrm{C}$ & -0.97071500 & 0.95393700 & -5.80347000 \\
\hline $\mathrm{C}$ & -2.25315800 & 1. 14943800 & -5.29007800 \\
\hline $\mathrm{C}$ & -3.57295300 & 0.67283400 & -5.85005200 \\
\hline $\mathrm{C}$ & -4.57475800 & 1. 00045500 & -4.72409400 \\
\hline $\mathrm{C}$ & -3.94048300 & 2. 19146800 & -3.93431900 \\
\hline $\mathrm{C}$ & -2.45363900 & 1. 93778500 & -4.14521300 \\
\hline $\mathrm{C}$ & -4.26963300 & 3. 53186900 & -4.67906600 \\
\hline $\mathrm{C}$ & -5.62062900 & 3. 97016100 & -4.12592500 \\
\hline $\mathrm{C}$ & -5.57572500 & 3. 44442500 & -2.71463400 \\
\hline $\mathrm{C}$ & -6.40500800 & 3. 89368600 & -1.68875800 \\
\hline $\mathrm{C}$ & -6.27645100 & 3.32076500 & -0.42995500 \\
\hline C & -5.33557500 & 2. 31045900 & -0.23090700 \\
\hline $\mathrm{C}$ & -4.48060400 & 1. 82763700 & -1.24583600 \\
\hline $\mathrm{C}$ & -4.59845900 & 2. 42954700 & -2.54087900 \\
\hline $\mathrm{C}$ & -3.92005400 & -0.95696200 & -1.81886000 \\
\hline $\mathrm{C}$ & -5.30713700 & -1.06228600 & -2.05157100 \\
\hline C & -5.83413700 & -2.08253500 & -2.84691800 \\
\hline $\mathrm{C}$ & -4.92566900 & -3.00219900 & -3.40832700 \\
\hline $\mathrm{C}$ & -3.54521700 & -2.93896100 & -3.19002100 \\
\hline $\mathrm{C}$ & -3.05178800 & -1.89400300 & -2.37928700 \\
\hline $\mathrm{C}$ & -2.58194700 & -4.00013700 & -3.75775000 \\
\hline $\mathrm{C}$ & -1.32917200 & -3.32419000 & -4.35658000 \\
\hline $\mathrm{C}$ & -3.23399900 & -4.85375000 & -4.86213200 \\
\hline $\mathrm{C}$ & -2.16803200 & -4.93508000 & -2.59673000 \\
\hline $\mathrm{C}$ & -7.34320100 & -2.22210800 & -3.13538000 \\
\hline $\mathrm{C}$ & -8.17324600 & -1.13712400 & -2.42586300 \\
\hline $\mathrm{C}$ & -7.58689900 & -2.09940700 & -4.65788700 \\
\hline $\mathrm{C}$ & -7.83629300 & -3.60429700 & -2.64783000 \\
\hline
\end{tabular}




\begin{tabular}{|c|c|c|c|}
\hline $\mathrm{C}$ & -4.04359600 & -0.15691300 & 0.94806700 \\
\hline $\mathrm{C}$ & -3.92387200 & 0.67033400 & 2. 07741800 \\
\hline $\mathrm{C}$ & -4.58343000 & 0.35896100 & 3. 28004200 \\
\hline $\mathrm{C}$ & -5.28175800 & -0.85558700 & 3. 34622200 \\
\hline $\mathrm{C}$ & -5.35329700 & -1.75143100 & 2. 26828400 \\
\hline $\mathrm{C}$ & -4.73380300 & -1.37499700 & 1. 06722900 \\
\hline $\mathrm{C}$ & -6.17886600 & -3.05175600 & 2. 37871200 \\
\hline $\mathrm{C}$ & -5.69999500 & -4.11948100 & 1. 37470000 \\
\hline $\mathrm{C}$ & -6.08526300 & -3.65845400 & 3. 79507800 \\
\hline $\mathrm{C}$ & -7.65812700 & -2.70987000 & 2. 07672500 \\
\hline $\mathrm{C}$ & -4.60165100 & 1. 36991800 & 4. 44768800 \\
\hline $\mathrm{C}$ & -5.42785900 & 2. 60474200 & 4. 01297700 \\
\hline $\mathrm{C}$ & -3.16932500 & 1. 81831300 & 4. 79288400 \\
\hline $\mathrm{C}$ & -5.23994600 & 0.78474000 & 5. 72113100 \\
\hline $\mathrm{C}$ & 0.52432900 & -2.96028400 & 2. 37741300 \\
\hline $\mathrm{C}$ & 1. 15227300 & -4.29727800 & 2. 81620400 \\
\hline $\mathrm{C}$ & 1. 66204300 & -3.88355800 & 0.66500300 \\
\hline $\mathrm{C}$ & 2. 34870200 & -4.21866700 & -0.60637800 \\
\hline $\mathrm{C}$ & 1.59072100 & -4.48589500 & -1.75783700 \\
\hline $\mathrm{C}$ & 2. 21311500 & -4.91413500 & -2.93173800 \\
\hline $\mathrm{C}$ & 3. 60560300 & -5.04644200 & -2.98832800 \\
\hline $\mathrm{C}$ & 4. 36579500 & -4.74284900 & -1.86011400 \\
\hline $\mathrm{C}$ & 5. 86745900 & -4.79384000 & -1.69166600 \\
\hline $\mathrm{C}$ & 6. 10470500 & -4.05393800 & -0.35721200 \\
\hline $\mathrm{C}$ & 4. 77817200 & -4.22781500 & 0.44788800 \\
\hline $\mathrm{C}$ & 3. 74501300 & -4.35177000 & -0.66270300 \\
\hline $\mathrm{C}$ & 4. 85480900 & -5.56997000 & 1. 25546500 \\
\hline $\mathrm{C}$ & 5. 46364000 & -5.17512700 & 2. 60485700 \\
\hline $\mathrm{C}$ & 4. 92032800 & -3.78057600 & 2. 80089600 \\
\hline $\mathrm{C}$ & 4. 73510900 & -3.13552100 & 4. 02027000 \\
\hline $\mathrm{C}$ & 4. 10469200 & -1.88949800 & 4. 03042500 \\
\hline $\mathrm{C}$ & 3. 73328700 & -1.29410700 & 2. 82712500 \\
\hline $\mathrm{C}$ & 3. 99269000 & -1.88806000 & 1. 56777000 \\
\hline $\mathrm{C}$ & 4. 54364600 & -3.19452700 & 1. 56638900 \\
\hline $\mathrm{C}$ & 4. 33987600 & -1.02988300 & -1.39078600 \\
\hline $\mathrm{C}$ & 5. 73023800 & -0.81725700 & -1.35651700 \\
\hline $\mathrm{C}$ & 6. 49302600 & -0.80290800 & -2.52990200 \\
\hline $\mathrm{C}$ & 5. 81212300 & -0.98894300 & -3.74514200 \\
\hline $\mathrm{C}$ & 4. 42765100 & -1.19098900 & -3.82423400 \\
\hline $\mathrm{C}$ & 3. 69812400 & -1.21453700 & -2.62094400 \\
\hline $\mathrm{C}$ & 3. 75945700 & -1.32063800 & -5.20936600 \\
\hline $\mathrm{C}$ & 2. 26075200 & -1.64997000 & -5.10005500 \\
\hline $\mathrm{C}$ & 4. 43779700 & -2.44148400 & -6.02970000 \\
\hline $\mathrm{C}$ & 3.91741300 & 0.02206600 & -5.96203200 \\
\hline $\mathrm{C}$ & 4. 10315800 & 0.88117600 & 0.60549000 \\
\hline
\end{tabular}




\begin{tabular}{|c|c|c|c|}
\hline $\mathrm{C}$ & 3. 70007200 & 1. 97086400 & -0.18215100 \\
\hline $\mathrm{C}$ & 4. 35589000 & 3. 20833700 & -0.09596200 \\
\hline $\mathrm{C}$ & 5. 39912800 & 3. 33347400 & 0.83624300 \\
\hline $\mathrm{C}$ & 5.83049900 & 2.27152400 & 1. 64321000 \\
\hline $\mathrm{C}$ & 5. 17275800 & 1. 03880500 & 1. 50019700 \\
\hline $\mathrm{C}$ & 7. 00103900 & 2. 47797800 & 2. 62849700 \\
\hline $\mathrm{C}$ & 7. 38777500 & 1. 17809300 & 3. 35751600 \\
\hline $\mathrm{C}$ & 8.24464800 & 2. 98706100 & 1. 86334800 \\
\hline $\mathrm{C}$ & 6.59348400 & 3.52441400 & 3. 69127000 \\
\hline $\mathrm{C}$ & 4. 00797100 & 4. 40683800 & -1.00165600 \\
\hline $\mathrm{C}$ & 0.11650000 & 1. 05206600 & 2. 37334900 \\
\hline $\mathrm{H}$ & -1.08002000 & 0.30678800 & -2.02702000 \\
\hline $\mathrm{H}$ & -0.85343800 & -0.87459200 & -0.12693500 \\
\hline $\mathrm{H}$ & 0.90104700 & 0.68438400 & -0.64909600 \\
\hline $\mathrm{H}$ & 1. 10582000 & -1.48168400 & -1.36526900 \\
\hline $\mathrm{H}$ & -2.30604400 & 3. 57301500 & 0.69974300 \\
\hline $\mathrm{H}$ & -3.51846400 & 4. 82282400 & -0.96077600 \\
\hline $\mathrm{H}$ & -2.14740300 & 5.98263700 & -0.88182600 \\
\hline $\mathrm{H}$ & 0.79600300 & 2. 73950800 & -3.49135400 \\
\hline $\mathrm{H}$ & 1. 12967500 & 1. 40895100 & -5.56315300 \\
\hline $\mathrm{H}$ & -0.82122200 & 0.35341100 & -6.70482000 \\
\hline $\mathrm{H}$ & -3.56513900 & -0.39793100 & -6.10760700 \\
\hline $\mathrm{H}$ & -3.80773500 & 1. 21666000 & -6.78243500 \\
\hline $\mathrm{H}$ & -4.67309000 & 0.13444700 & -4.06296500 \\
\hline $\mathrm{H}$ & -5.58062500 & 1. 24571100 & -5.09655200 \\
\hline $\mathrm{H}$ & -3.50748900 & 4. 28013300 & -4.41842600 \\
\hline $\mathrm{H}$ & -4.25184200 & 3. 40379700 & -5.77096500 \\
\hline $\mathrm{H}$ & -5.77718600 & 5. 05932700 & -4.16758200 \\
\hline $\mathrm{H}$ & -6.46044300 & 3.50901800 & -4.67630400 \\
\hline $\mathrm{H}$ & -7.13845100 & 4. 68121400 & -1.88076900 \\
\hline $\mathrm{H}$ & -6.90646700 & 3. 64198900 & 0.40294300 \\
\hline $\mathrm{H}$ & -5.28918300 & 1. 87124400 & 0.76013700 \\
\hline $\mathrm{H}$ & -5.97338500 & -0.32618400 & -1.60540000 \\
\hline $\mathrm{H}$ & -5.32226700 & -3.79929400 & -4.03571400 \\
\hline $\mathrm{H}$ & -1.98381400 & -1.80593900 & -2.18091700 \\
\hline $\mathrm{H}$ & -0.74998300 & -2.76486600 & -3.60785600 \\
\hline $\mathrm{H}$ & -1.60413000 & -2.62134200 & -5.15887500 \\
\hline $\mathrm{H}$ & -0.66040900 & -4.08427900 & -4.79118600 \\
\hline $\mathrm{H}$ & -4.07885600 & -5.45104200 & -4.48711300 \\
\hline $\mathrm{H}$ & -2.49590700 & -5.56221500 & -5.26930900 \\
\hline $\mathrm{H}$ & -3.59668900 & -4.23385200 & -5.69736200 \\
\hline $\mathrm{H}$ & -1.70423300 & -4.36802800 & -1.77478000 \\
\hline $\mathrm{H}$ & -1.44814500 & -5.69539700 & -2.94161300 \\
\hline $\mathrm{H}$ & -3.04364600 & -5.45968100 & -2.18346100 \\
\hline $\mathrm{H}$ & -8.06364500 & -1.18408900 & -1.33107600 \\
\hline
\end{tabular}




\begin{tabular}{|c|c|c|c|}
\hline H & -9.24084700 & -1.27658200 & -2.65515800 \\
\hline $\mathrm{H}$ & -7.89601700 & -0.12323900 & -2.75387200 \\
\hline $\mathrm{H}$ & -7.25256100 & -1.12006200 & -5.03583900 \\
\hline $\mathrm{H}$ & -8.66091700 & -2.19965500 & -4.88305100 \\
\hline $\mathrm{H}$ & -7.05586500 & -2.87860100 & -5.22560300 \\
\hline H & -7.68226800 & -3.72086900 & -1.56335700 \\
\hline $\mathrm{H}$ & -7.31475900 & -4.43187900 & -3.15215500 \\
\hline H & -8.91313600 & -3.71980200 & -2.85062200 \\
\hline $\mathrm{H}$ & -3.33356800 & 1.58398300 & 2. 00452200 \\
\hline $\mathrm{H}$ & -5.79280200 & -1.11582000 & 4. 26965800 \\
\hline H & -4.80144300 & -2.03532300 & 0.20816800 \\
\hline $\mathrm{H}$ & -5.84104200 & -3.80855200 & 0.32875900 \\
\hline H & -6.27649300 & -5.04705200 & 1. 51510000 \\
\hline $\mathrm{H}$ & -4.63597800 & -4.36187600 & 1. 52208200 \\
\hline $\mathrm{H}$ & -6.63188900 & -4.61382100 & 3. 82793400 \\
\hline $\mathrm{H}$ & -6.53355700 & -3.00917100 & 4. 56182300 \\
\hline H & -5.04166800 & -3.85577800 & 4. 08190800 \\
\hline H & -7.77324300 & -2.29966600 & 1. 06075200 \\
\hline $\mathrm{H}$ & -8.04852700 & -1.96280100 & 2. 78559200 \\
\hline H & -8.28793300 & -3.61150300 & 2. 15369200 \\
\hline $\mathrm{H}$ & -6.45866700 & 2. 31926600 & 3. 74876300 \\
\hline H & -4.98060600 & 3. 10646600 & 3. 14037300 \\
\hline $\mathrm{H}$ & -5.47958500 & 3.34224400 & 4. 83058500 \\
\hline $\mathrm{H}$ & -2.55338000 & 0.96684700 & 5. 12025200 \\
\hline $\mathrm{H}$ & -3.18327400 & 2.55913300 & 5. 60834800 \\
\hline $\mathrm{H}$ & -2.67123600 & 2. 28080300 & 3. 92984800 \\
\hline $\mathrm{H}$ & -4.71187600 & -0.11863800 & 6. 06382400 \\
\hline $\mathrm{H}$ & -6.30091200 & 0.52978600 & 5. 57624000 \\
\hline $\mathrm{H}$ & -5.19304500 & 1.52710800 & 6. 53289300 \\
\hline $\mathrm{H}$ & 0.89158100 & -2.12795500 & 2. 98981200 \\
\hline $\mathrm{H}$ & 1. 99949100 & -4.16715700 & 3. 50511400 \\
\hline $\mathrm{H}$ & 0.44045900 & -5.01385600 & 3. 24250300 \\
\hline $\mathrm{H}$ & 0.50720800 & -4.37868200 & -1.72187200 \\
\hline $\mathrm{H}$ & 1. 60559500 & -5.14391300 & -3.80985700 \\
\hline $\mathrm{H}$ & 4. 09007200 & -5.38039900 & -3.90964800 \\
\hline $\mathrm{H}$ & 6. 39959900 & -4.32444900 & -2.53380500 \\
\hline $\mathrm{H}$ & 6. 21258900 & -5.84201400 & -1.64485600 \\
\hline $\mathrm{H}$ & 6. 97286600 & -4.43597300 & 0.19995300 \\
\hline $\mathrm{H}$ & 6.28121500 & -2.99168900 & -0.55473300 \\
\hline $\mathrm{H}$ & 3. 83958700 & -5.95983900 & 1. 41068200 \\
\hline $\mathrm{H}$ & 5. 43027500 & -6.33632000 & 0.71568500 \\
\hline $\mathrm{H}$ & 5. 19089200 & -5.85941100 & 3. 42295100 \\
\hline $\mathrm{H}$ & 6.56771200 & -5.15516500 & 2. 56174200 \\
\hline $\mathrm{H}$ & 5. 03897100 & -3.61758100 & 4. 95324200 \\
\hline . & 3.88937800 & -1.38177900 & 4. 97386300 \\
\hline
\end{tabular}




\begin{tabular}{|c|c|c|c|}
\hline $\mathrm{H}$ & 3. 20462300 & -0.34206700 & 2. 86372700 \\
\hline $\mathrm{H}$ & 6.21581000 & -0.63277400 & -0.40014900 \\
\hline $\mathrm{H}$ & 6.38770400 & -0.95578100 & -4.67173600 \\
\hline $\mathrm{H}$ & 2. 61926100 & -1.35345000 & -2.62781100 \\
\hline H & 1. 69746400 & -0.86493900 & -4.57499700 \\
\hline $\mathrm{H}$ & 2. 09276700 & -2.59825900 & -4.56926100 \\
\hline $\mathrm{H}$ & 1.82716800 & -1.74821600 & -6.10788700 \\
\hline $\mathrm{H}$ & 5.50845700 & -2.24971400 & -6.19607800 \\
\hline $\mathrm{H}$ & 3. 96318000 & -2.52882400 & -7.02043200 \\
\hline $\mathrm{H}$ & 4. 34409400 & -3.41394400 & -5.52259300 \\
\hline $\mathrm{H}$ & 3. 46799900 & 0.85025300 & -5.39115900 \\
\hline $\mathrm{H}$ & 3. 42393100 & -0.02525800 & -6.94688600 \\
\hline $\mathrm{H}$ & 4. 97525600 & 0.27443100 & -6.13249000 \\
\hline $\mathrm{H}$ & 2. 88509500 & 1. 82926000 & -0.89049000 \\
\hline $\mathrm{H}$ & 5. 90939000 & 4. 29441700 & 0.92143000 \\
\hline $\mathrm{H}$ & 5.51282900 & 0.18801100 & 2. 08307400 \\
\hline $\mathrm{H}$ & 6.55756000 & 0.77554900 & 3. 95827900 \\
\hline $\mathrm{H}$ & 8.22548400 & 1. 37302700 & 4. 04469600 \\
\hline $\mathrm{H}$ & 7. 71388300 & 0.39387300 & 2. 65645000 \\
\hline $\mathrm{H}$ & 8.55808900 & 2. 26673300 & 1. 09131200 \\
\hline $\mathrm{H}$ & 9.08908700 & 3. 12727000 & 2. 55714900 \\
\hline $\mathrm{H}$ & 8.06283700 & 3. 95264400 & 1. 36799600 \\
\hline $\mathrm{H}$ & 5. 72987900 & 3. 17410700 & 4. 27852700 \\
\hline $\mathrm{H}$ & 6.32496600 & 4. 49037800 & 3. 23637300 \\
\hline $\mathrm{H}$ & 7. 42446900 & 3. 70641800 & 4. 39189400 \\
\hline $\mathrm{C}$ & 5. 25447300 & 4. 79665900 & -1.83123400 \\
\hline $\mathrm{H}$ & 5. 02026200 & 5.63995900 & -2.50116700 \\
\hline $\mathrm{H}$ & 6.09588700 & 5. 10547600 & -1.19296300 \\
\hline $\mathrm{H}$ & 5.59510100 & 3. 95268100 & -2.45192800 \\
\hline $\mathrm{C}$ & 2.87225500 & 4. 07465500 & -1.98503400 \\
\hline $\mathrm{H}$ & 3. 15325500 & 3. 25669100 & -2.66680800 \\
\hline $\mathrm{H}$ & 1. 94988200 & 3. 77897600 & -1.46333200 \\
\hline $\mathrm{H}$ & 2. 64190900 & 4. 95623100 & -2.60376800 \\
\hline $\mathrm{C}$ & 3.57650700 & 5. 60892300 & -0.13136100 \\
\hline $\mathrm{H}$ & 3. 31740100 & 6.47317700 & -0.76387600 \\
\hline $\mathrm{H}$ & 2. 69668300 & 5. 36317200 & 0.48336000 \\
\hline $\mathrm{H}$ & 4. 37855200 & 5.93009000 & 0.55028600 \\
\hline $\mathrm{C}$ & 8.01592100 & -0.55505900 & -2.53537700 \\
\hline $\mathrm{C}$ & 8.58965600 & -0.42344600 & -1.11301700 \\
\hline $\mathrm{H}$ & 8. 15323000 & 0.42955800 & -0.57087400 \\
\hline $\mathrm{H}$ & 8.42176100 & -1.33305600 & -0.51486200 \\
\hline $\mathrm{H}$ & 9. 67718800 & -0.25985600 & -1.16365700 \\
\hline $\mathrm{C}$ & 8. 73325800 & -1.73088000 & -3.23789000 \\
\hline $\mathrm{H}$ & 8. 40547000 & -1.85321200 & -4.28129700 \\
\hline & 9.82204300 & -1.56201200 & -3.2519100 \\
\hline
\end{tabular}




\begin{tabular}{|c|c|c|c|}
\hline H & 8.54645500 & -2.68118600 & -2.71264300 \\
\hline $\mathrm{C}$ & 8.31170000 & 0.75669000 & -3.30013500 \\
\hline $\mathrm{H}$ & 7. 96813000 & 0.70990800 & -4.34482600 \\
\hline $\mathrm{H}$ & 7. 81368200 & 1. 61422600 & -2.82024900 \\
\hline $\mathrm{H}$ & 9. 39536400 & 0.95675100 & -3.31541600 \\
\hline $\mathrm{C}$ & 0.32950100 & 1. 71365400 & 3. 72823100 \\
\hline $\mathrm{H}$ & -0.56727500 & 2. 27618900 & 4. 01205200 \\
\hline $\mathrm{H}$ & 0.48188300 & 0.93347500 & 4. 48789800 \\
\hline $\mathrm{C}$ & 1. 54360900 & 2. 64696800 & 3. 74868200 \\
\hline $\mathrm{H}$ & 1. 40993500 & 3.50880600 & 3. 07378300 \\
\hline $\mathrm{H}$ & 2. 44770100 & 2. 12533200 & 3. 38288700 \\
\hline $\mathrm{C}$ & 1. 86273700 & 3. 15640500 & 5. 15262800 \\
\hline 0 & 1. 42452300 & 2. 60716100 & 6. 13945800 \\
\hline $\mathrm{C}$ & 2. 74743600 & 4. 38289300 & 5. 23932800 \\
\hline $\mathrm{H}$ & 3.59389400 & 4. 32232600 & 4. 53769100 \\
\hline $\mathrm{H}$ & 2. 15498100 & 5. 26868300 & 4. 95095400 \\
\hline $\mathrm{H}$ & 3. 10841300 & 4. 51568000 & 6. 26770700 \\
\hline $\mathrm{C}$ & -0.37086000 & 4. 52492600 & 0.48371200 \\
\hline $\mathrm{H}$ & 0.14685300 & 3. 69536600 & 0.98182800 \\
\hline $\mathrm{H}$ & 0.27516600 & 4.85736200 & -0.33907700 \\
\hline $\mathrm{C}$ & -1.01910800 & -2.93965900 & 2. 39960600 \\
\hline $\mathrm{H}$ & -1.35260400 & -1.95407500 & 2. 04445800 \\
\hline $\mathrm{H}$ & -1.40221000 & -3.67498100 & 1. 67846100 \\
\hline $\mathrm{C}$ & -1.55781000 & -3.18238600 & 3. 79815400 \\
\hline $\mathrm{C}$ & -2.11645500 & -4.44285000 & 4. 20874000 \\
\hline $\mathrm{C}$ & -1.45693500 & -2.16040300 & 4. 73015600 \\
\hline $\mathrm{C}$ & -2.27075500 & -5.56014600 & 3. 33308400 \\
\hline $\mathrm{C}$ & -2.54844000 & -4.60219300 & 5. 57445500 \\
\hline $\mathrm{C}$ & -1.88015700 & -2.31979900 & 6. 07020700 \\
\hline $\mathrm{H}$ & -1.03814400 & -1.19960100 & 4. 41984700 \\
\hline $\mathrm{C}$ & -2.81003900 & -6.75261400 & 3. 77142000 \\
\hline $\mathrm{H}$ & -1.96038800 & -5.47816200 & 2. 29071400 \\
\hline $\mathrm{C}$ & -3.10200200 & -5.84618300 & 5. 99181600 \\
\hline $\mathrm{C}$ & -2.41548700 & -3.51892800 & 6. 48538400 \\
\hline $\mathrm{H}$ & -1.77673300 & -1.48762800 & 6. 77095100 \\
\hline $\mathrm{C}$ & -3.23121100 & -6.90111100 & 5. 11521100 \\
\hline $\mathrm{H}$ & -2.91495900 & -7.58868100 & 3. 07549400 \\
\hline $\mathrm{H}$ & -3.42454700 & -5.94952900 & 7. 03136500 \\
\hline $\mathrm{H}$ & -2.74365300 & -3.65564600 & 7. 51909300 \\
\hline $\mathrm{H}$ & -3.65683800 & -7.84954500 & 5. 45148000 \\
\hline $\mathrm{C}$ & -0.62196100 & 5. 64363500 & 1. 47809900 \\
\hline $\mathrm{C}$ & -0.40662900 & 7. 03028000 & 1. 16074500 \\
\hline $\mathrm{C}$ & -1.09744100 & 5. 31002800 & 2. 73817600 \\
\hline $\mathrm{C}$ & 0.07173600 & 7. 48609200 & -0.10486900 \\
\hline $\mathrm{C}$ & -0.68526300 & 8.02510500 & 2. 16554200 \\
\hline
\end{tabular}




$\begin{array}{rrrr}\mathrm{C} & -1.37254800 & 6.28914600 & 3.72124800 \\ \mathrm{H} & -1.26065300 & 4.25618200 & 2.97674500 \\ \mathrm{C} & 0.26068100 & 8.82834600 & -0.36426300 \\ \mathrm{H} & 0.29799100 & 6.76563200 & -0.89177100 \\ \mathrm{C} & -0.47490400 & 9.40102800 & 1.86464300 \\ \mathrm{C} & -1.16791900 & 7.62194000 & 3.44048800 \\ \mathrm{H} & -1.74443300 & 5.98082800 & 4.70142800 \\ \mathrm{C} & -0.01377700 & 9.79939900 & 0.62928000 \\ \mathrm{H} & 0.62694400 & 9.14501000 & -1.34404900 \\ \mathrm{H} & -0.69002600 & 10.14202500 & 2.63917800 \\ \mathrm{H} & -1.37509300 & 8.38812800 & 4.19212700 \\ \mathrm{H} & 0.14105900 & 10.85909900 & 0.41305300\end{array}$




\section{References}

[1] Zhu, S.-F.; Zhou, Q.-L. Iridium-Catalyzed Asymmetric Hydrogenation of Unsaturated Carboxylic Acids. Acc. Chem. Res. 2017, 50, 988-1001.

[2] Singh, S.; Verma, M.; Singh, K. N. Superoxide Ion Induced Oxidation of $\gamma$-Lactones to $\gamma$ Ketocarboxylic Acids. Synth. Commun. 2004, 34, 4471-4475.

[3] Betancourt de Perez, R. M.; Fuentes, L. M.; Larson, G. L.; Barnes, C. L.; Heeg, M. J. The Chemistry Of .Alpha.-Silyl Carbonyl Compounds. 12. A Synthesis of 4-Oxo Carboxylic Acids, 4Oxo Aldehydes, and 1,4-Diketones From .Gamma.-Lactones. J. Org. Chem. 1986, 51, 2039-2043.

[4] Starodubtseva, E. V.; Turova, O. V.; Vinogradov, M. G.; Gorshkova, L. S.; Ferapontov, V. A.; Struchkova, M. I. A Convenient Route to Chiral $\Gamma$-Lactones via Asymmetric Hydrogenation of $\gamma$ Ketoesters Using the $\mathrm{RuCl}_{3}$-Binap-HCl Catalytic System. Tetrahedron 2008, 64, 11713-11717.

[5] Shinkai, H.; Ozeki, H.; Motomura, T.; Ohta, T.; Furukawa, N.; Uchida, I. 4-(trans-4Methylcyclohexyl)-4-Oxobutyric Acid (JTT-608). A New Class of Antidiabetic Agent. J. Med. Chem. 1998, 41, 5420-5428.

[6] Baxter, E. W.; Conway, K. A.; Kennis, L.; Bischoff, F.; Mercken, M. H.; De Winter, H. L.; Reynolds, C. H.; Tounge, B. A.; Luo, C.; Scott, M. K.; Huang, Y.; Braeken, M.; Pieters, S. M. A.; Berthelot, D. J. C.; Masure, S.; Bruinzeel, W. D.; Jordan, A. D.; Parker, M. H.; Boyd, R. E.; Qu, J.; Alexander, R. S.; Brenneman, D. E.; Reitz, A. B. 2-Amino-3,4-Dihydroquinazolines as Inhibitors of Bace-1 ( $\beta$-Site App Cleaving Enzyme): Use of Structure Based Design to Convert a Micromolar Hit into a Nanomolar Lead. J. Med. Chem. 2007, 50, 4261-4264.

[7] Ketterer, C.; Wünsch, B. Lipase-Catalyzed Kinetic Resolution of 2-Phenylethanol Derivatives and Chiral Oxa-Pictet-Spengler Reaction as the Key Steps in the Synthesis of Enantiomerically Pure Tricyclic Amines. Eur. J. Org. Chem. 2012, 2012, 2428-2444.

[8] Mitschka, R.; Oehldrich, J.; Takahashi, K.; Cook, J. M.; Weiss, U.; Silverton, J. V. General Approach for the Synthesis of Polyquinanes. Facile Generation of Molecular Complexity via Reaction of 1,2-Dicarbonyl Compounds with Dimethyl 3. Tetrahedron 1981, 37, 4521-4542.

[9] Maljutenko, K.; Borovkov, V.; Kananovich, D.; Järving, I.; Lopp, M. Aerobic Cascade Oxidation of Substituted Cyclopentane-1,2-Diones Using Metalloporphyrin Catalysts. Tetrahedron 2018, 74, 661-664.

[10] Versteegen, R. M.; van Beek, D. J. M.; Sijbesma, R. P.; Vlassopoulos, D.; Fytas, G.; Meijer, E. W. Dendrimer-Based Transient Supramolecular Networks. J. Am. Chem. Soc. 2005, 127, 13862-13868.

[11] Schwartz, B. D.; Tilly, D. P.; Heim, R.; Wiedemann, S.; Williams, C. M.; Bernhardt, P. V. Towards the Total Synthesis of Vibsanin E, 15-O-Methylcyclovibsanin B,3-Hydroxyvibsanin E, Furanovibsanin a, and 3-O-Methylfuranovibsanin A. Eur. J. Org. Chem. 2006, 2006, 3181-3192.

[12] Chatupheeraphat, A.; Soorukram, D.; Kuhakarn, C.; Tuchinda, P.; Reutrakul, V.; Pakawatchai, C.; Saithong, S.; Pohmakotr, M. Synthesis of Gem-Difluoromethylenated Spiro- $\gamma$-Butyrolactones by Employing $\mathrm{PhSCF}_{2} \mathrm{Si}\left(\mathrm{CH}_{3}\right)_{3}$ as a Gem-Difluoromethylenating Agent. Eur. J. Org. Chem. 2013, 2013, 6844-6858.

[13] Lee, J.; Sharma, R.; Wang, S.; Milne, G. W. A.; Lewin, N. E.; Szallasi, Z.; Blumberg, P. M.; George, C.; Marquez, V. E. Conformationally Constrained Analogues of Diacylglycerol. 12. Ultrapotent Protein Kinase C Ligands Based on a Chiral 4,4-Disubstituted Heptono-1,4-Lactone Template. J. Med. Chem. 1996, 39, 36-45.

[14] Nájera, C.; Yus, M.; Seebach, D. C-Metallierte Chirale Aloxide Als d²- Und d ${ }^{3}$-Reagenzien Für Die Synthese Enantiomerenreiner Produkte (Epc-Synthese). Helv. Chim. Acta 1984, 67, 289-300.

[15] Arceo, E.; Odriozola, J. M.; García, J. M.; González, A.; Gil, P. Asymmetric Synthesis of (R)-4Hexanolide, the Pheromone of Trogoderma Glabrum. Tetrahedron: Asymmetry 2003, 14, 1617 1621.

[16] Yadav, J. S.; Maniyan, P. P. A Convenient Chiral Synthesis of 4-Alkyl- $\gamma$-Butanolides. Synth. Commun. 1993, 23, 2731-2741.

[17] Haynes, S. W.; Sydor, P. K.; Corre, C.; Song, L.; Challis, G. L. Stereochemical Elucidation of Streptorubin B. J. Am. Chem. Soc. 2011, 133, 1793-1798.

[18] Thijs, L.; Waanders, P. P.; Stokkingreef, E. H. M.; Zwanenburg, B. Synthesis of the Optical Antipodes of 4-Alkyl- $\gamma$-Lactones. Recl. Trav. Chim. Pays-Bas 1986, 105, 332-337. 
[19] Barluenga, J.; Fernandez, J. R.; Rubiera, C.; Yus, M. 3,3-Diethoxypropyllithium. A Masked Lithium Propanal Homoenolate in Organic Synthesis. J. Chem. Soc., Perkin Trans. 1 1988, $3113-$ 3117.

[20] Jha, V.; Kondekar, N. B.; Kumar, P. Enantioselective Synthesis of Syn/Anti-1,3-Amino Alcohols Via Proline-Catalyzed Sequential $\alpha$-Aminoxylation $/ \alpha$-Amination $\quad$ and Horner-Wadsworth-Emmons Olefination of Aldehydes. Org. Lett. 2010, 12, 2762-2765.

[21] Vogel, J. C.; Butler, R.; Procter, D. J. An Asymmetric, $\mathrm{SmI}_{2}-$ Mediated Approach to $\gamma$ Butyrolactones Using a New, Fluorous-Tagged Auxiliary. Tetrahedron 2008, 64, 11876-11883.

[22] Kerrigan, N. J.; Hutchison, P. C.; Heightman, T. D.; Procter, D. J. Development of a Solid-Phase Asymmetric Resin-Capture-Release' Process: Application of an Ephedrine Chiral Resin in an Approach to Г-Butyrolactones. Org. Biomol. Chem. 2004, 2, 2476-2482.

[23] Kotkar, S. P.; Suryavanshi, G. S.; Sudalai, A. A Short Synthesis of (+)-Harzialactone a and $(R)-$ $(+)-4-H e x a n o l i d e ~ V i a$ Proline-Catalyzed Sequential $\alpha$-Aminooxylation and Horner-WadsworthEmmons Olefination of Aldehydes. Tetrahedron: Asymmetry 2007, 18, 1795-1798.

[24] Shimotori, Y.; Hoshi, M.; Inoue, K.; Osanai, T.; Okabe, H.; Miyakoshi, T. Preparation of Optically Active 4-Substituted $\Gamma$-Lactones by Lipase-Catalyzed Optical Resolution. Heterocycl. Commun. 2015, 21, 165-174.

[25] Gutman, A. L.; Zuobi, K.; Bravdo, T. Lipase-Catalyzed Preparation of Optically Active .Gamma.Butyrolactones in Organic Solvents. J. Org. Chem. 1990, 55, 3546-3552.

[26] Murphy, S. K.; Dong, V. M. Enantioselective Ketone Hydroacylation Using Noyori's Transfer Hydrogenation Catalyst. J. Am. Chem. Soc. 2013, 135, 5553-5556.

[27] Qi, J.; Sun, C.; Tian, Y.; Wang, X.; Li, G.; Xiao, Q.; Yin, D. Highly Efficient and Versatile Synthesis of Lactams and $\mathrm{N}$-Heterocycles via $\mathrm{Al}(\mathrm{OTf})_{3}$-Catalyzed Cascade Cyclization and Ionic Hydrogenation Reactions. Org. Lett. 2014, 16, 190-192.

[28] Li, M.-L.; Yang, S.; Su, X.-C.; Wu, H.-L.; Yang, L.-L.; Zhu, S.-F.; Zhou, Q.-L. Mechanism Studies of Ir-Catalyzed Asymmetric Hydrogenation of Unsaturated Carboxylic Acids. J. Am. Chem. Soc. 2017, 139, 541-547.

[29] Gruber, S.; Pfaltz, A. Asymmetric Hydrogenation with Iridium C,N and N,P Ligand Complexes: Characterization of Dihydride Intermediates with a Coordinated Alkene. Angew. Chem. Int. Ed. 2014, 53, 1896-1900.

[30] Gaussian 09, Revision D.01, Frisch, M. J.; Trucks, G. W.; Schlegel, H. B.; Scuseria, G. E.; Robb, M. A.; Cheeseman, J. R.; Scalmani, G.; Barone, V.; Petersson, G. A.; Nakatsuji, H.; Li, X.; Caricato, M.; Marenich, A. V.; Bloino, J.; Janesko, B. G.; Gomperts, R.; Mennucci, B.; Hratchian, H. P.; Ortiz, J. V.; Izmaylov, A. F.; Sonnenberg, J. L.; Williams-Young, D.; Ding, F.; Lipparini, F.; Egidi, F.; Goings, J.; Peng, B.; Petrone, A.; Henderson, T.; Ranasinghe, D.; Zakrzewski, V. G.; Gao, J.; Rega, N.; Zheng, G.; Liang, W.; Hada, M.; Ehara, M.; Toyota, K.; Fukuda, R.; Hasegawa, J.; Ishida, M.; Nakajima, T.; Honda, Y.; Kitao, O.; Nakai, H.; Vreven, T.; Throssell, K.; Montgomery, J. A., Jr.; Peralta, J. E.; Ogliaro, F.; Bearpark, M. J.; Heyd, J. J.; Brothers, E. N.; Kudin, K. N.; Staroverov, V. N.; Keith, T. A.; Kobayashi, R.; Normand, J.; Raghavachari, K.; Rendell, A. P.; Burant, J. C.; Iyengar, S. S.; Tomasi, J.; Cossi, M.; Millam, J. M.; Klene, M.; Adamo, C.; Cammi, R.; Ochterski, J. W.; Martin, R. L.; Morokuma, K.; Farkas, O.; Foresman, J. B.; Fox, D. J. Gaussian, Inc., Wallingford CT, 2016.

[31] Lee, C.; Yang, W.; Parr, R. G. Development of the Colle-Salvetti Correlation-Energy Formula into a Functional of the Electron Density. Phys. Rev. B 1988, 37, 785-789.

[32] Becke, A. D. A New Mixing of Hartree-Fock and Local Density-Functional Theories. J. Chem. Phys. 1993, 98, 1372-1377.

[33] Becke, A. D. Density-Functional Thermochemistry. III. The Role of Exact Exchange. J. Chem. Phys. 1993, 98, 5648-5652.

[34] Weigend, F.; Ahlrichs, R. Balanced Basis Sets of Split Valence, Triple Zeta Valence and Quadruple Zeta Valence Quality for $\mathrm{H}$ to Rn: Design and Assessment of Accuracy. Phys. Chem. Chem. Phys. 2005, 7, 3297-3305.

[35] Weigend, F. Accurate Coulomb-Fitting Basis Sets for $\mathrm{H}$ to Rn. Phys. Chem. Chem. Phys. 2006, 8, 1057-1065. 
[36] Marenich, A. V.; Cramer, C. J.; Truhlar, D. G. Universal Solvation Model Based on Solute Electron Density and on a Continuum Model of the Solvent Defined by the Bulk Dielectric Constant and Atomic Surface Tensions. J. Phys. Chem. B 2009, 113, 6378-6396.

[37] Grimme, S.; Antony, J.; Ehrlich, S.; Krieg, H. A Consistent and Accurate ab Initio Parametrization of Density Functional Dispersion Correction (DFT-D) for the 94 Elements H-Pu. J. Chem. Phys. 2010, 132, 154104.

[38] CYLview, 1.0b; Legault, C. Y. Université de Sherbrooke, 2009. (http://www.cylview.org). 